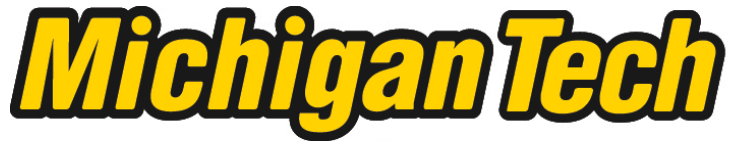 \\ Michigan Technological University Create the Future Digital Commons @ Michigan Tech
}

\section{A Study of Acoustic Boom Generation Due to Vibro-Acoustic Coupling in Passenger Cars}

Mayuresh Pathak

Michigan Technological University

Follow this and additional works at: https://digitalcommons.mtu.edu/etds

Part of the Mechanical Engineering Commons

Copyright 2014 Mayuresh Pathak

\section{Recommended Citation}

Pathak, Mayuresh, "A Study of Acoustic Boom Generation Due to Vibro-Acoustic Coupling in Passenger Cars", Master's Thesis, Michigan Technological University, 2014.

https://doi.org/10.37099/mtu.dc.etds/851

Follow this and additional works at: https://digitalcommons.mtu.edu/etds

Part of the Mechanical Engineering Commons 


\title{
A STUDY OF ACOUSTIC CAVITY BOOM GENERATION DUE TO VIBRO- ACOUSTIC COUPLING IN PASSENGER CARS
}

\author{
By
}

Mayuresh Pathak

\section{A THESIS}

Submitted in partial fulfillment of the requirements for the degree of MASTER OF SCIENCE

In Mechanical Engineering

MICHIGAN TECHNOLOGICAL UNIVERSITY

2014

(c) 2014 Mayuresh Pathak 
This thesis has been approved in partial fulfillment of the requirements for the Degree of MASTER OF SCIENCE in Mechanical Engineering

Department of Mechanical Engineering-Engineering Mechanics

Thesis Advisor: $\quad$ Prof. Charles D. Van Karsen

Committee Member: Dr. James De Clerck

Committee Member: Dr. Gregory Odegard

Committee Member: $\quad$ Prof. John Irwin

Department Chair: Dr. William W. Predebon 


\section{Table of Contents}

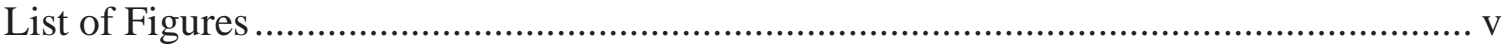

Acknowledgements ............................................................................................... xi

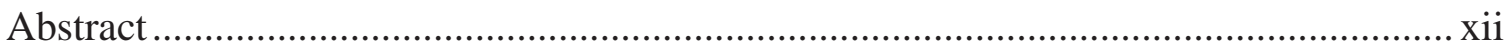

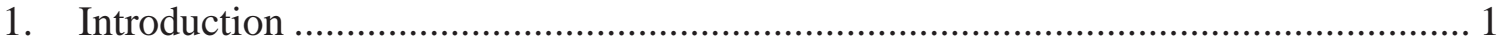

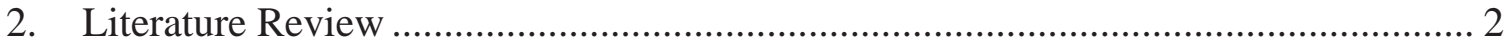

3. Tests Performed on the Chevrolet Cruze LTZ ......................................................... 6

$3.1 \quad$ Impact Hammer Test .................................................................................. 6

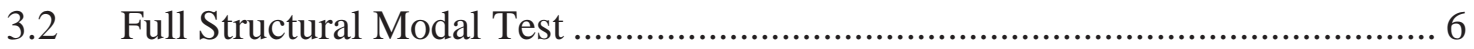

3.3 Full Acoustic Modal Test - Structural Input................................................. 6

3.4 Full Acoustic Modal Test - Acoustic Input …………….................................. 6

3.5 Full Acoustic Modal Test - Structural Input: Door Cavity Testing ..................... 6

3.6 Full Acoustic Modal Test- Structural Input: Effect of Rear Seats and Package

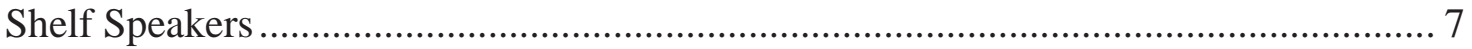

3.7 Full Acoustic Modal Test - Structural Input: Seatback Movement Testing........ 7

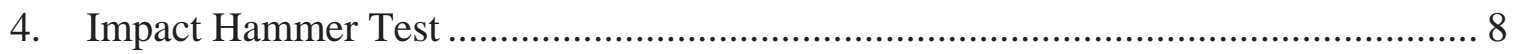

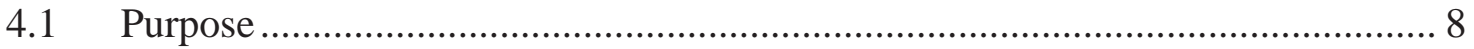

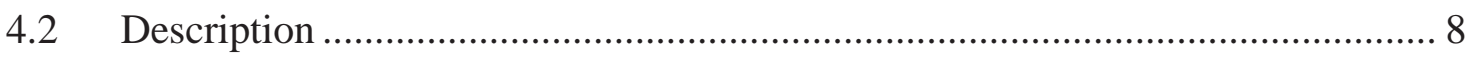

4.3 Summary of Impact Test ........................................................................ 12

5. Full Structural Modal Test............................................................................... 13

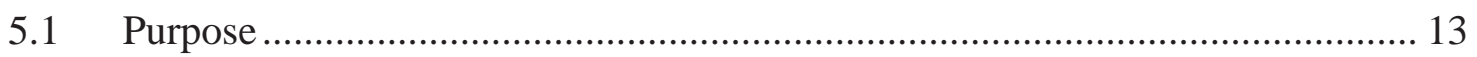

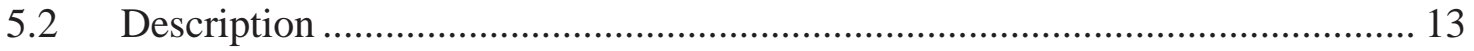

5.3. Summary of Structural Modal Test................................................................ 29

6. Full Acoustic Modal Test - Structural Input .......................................................... 30

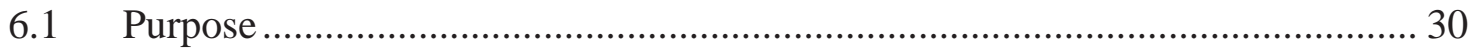

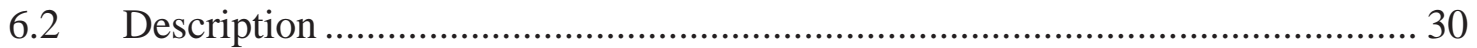

6.3 Summary of the Acoustic Modal Test with Structural Input .............................. 36

7. Full Acoustic Modal Test: Acoustic Input ............................................................. 37

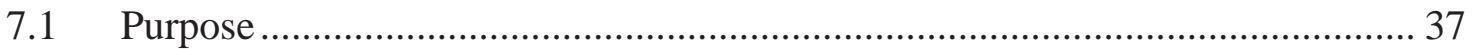

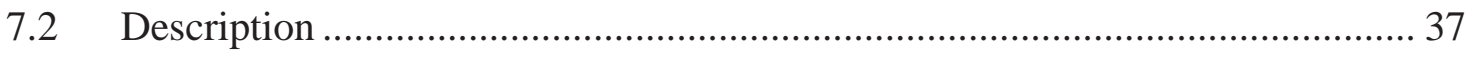


7.3 Summary of Acoustic Modal Test with Acoustic Excitation........................... 44

8. Full Acoustic Modal Test - Structural Input: Door Cavity Testing ........................ 45

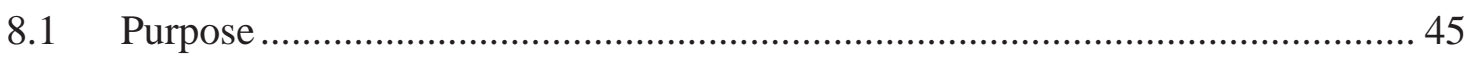

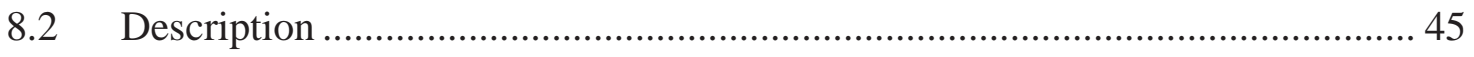

8.3 Summary of Door Cavity Testing ............................................................... 59

9. Full Acoustic Modal Test - Structural Input: Seatback Movement Testing ........... 60

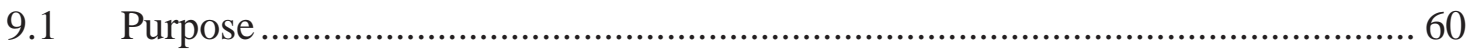

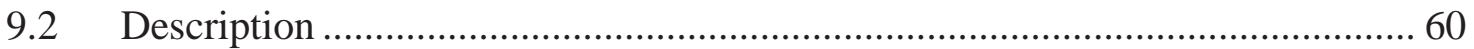

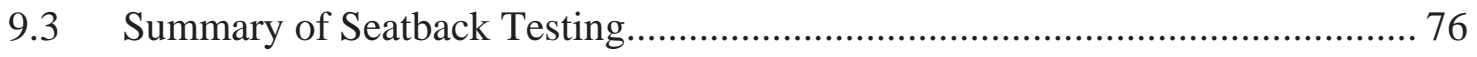

10. Understanding Vibro-Acoustic behavior through Simple Car Models................. 77

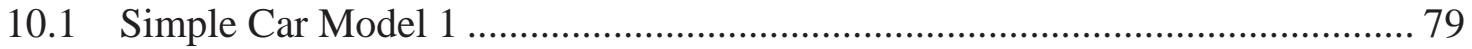

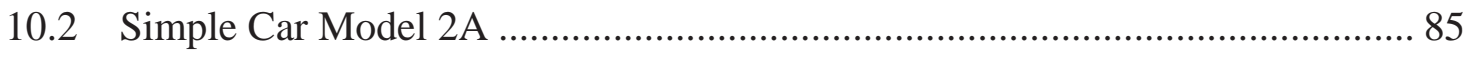

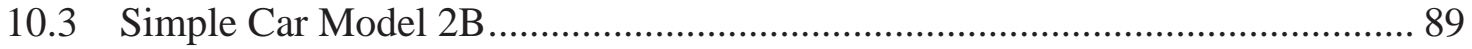

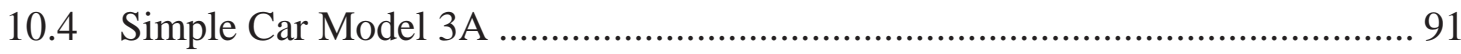

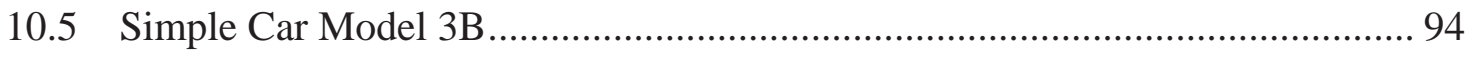

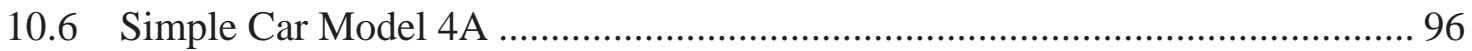

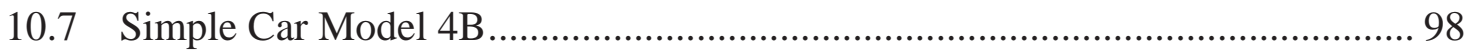

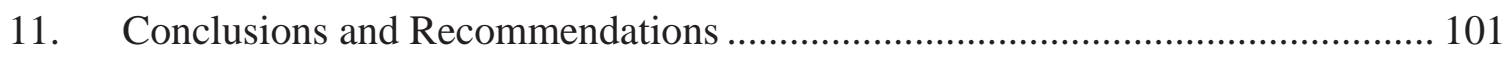

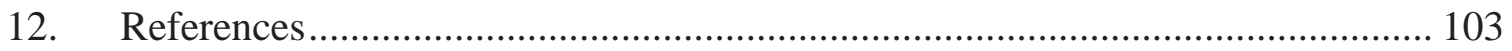




\section{List of Figures}

Figure 1: Fluid Structure Interaction in Coupled Systems................................................... 3

Figure 2: Impact Locations for Impact Hammer Testing .................................................. 8

Figure 3: Acoustic Response Locations for Impact Hammer Testing ............................... 9

Figure 4: Zoomed View of Structural Response Locations (Header Center and A Pillar

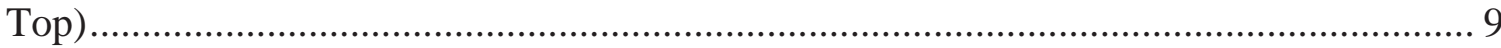

Figure 5: FRF and Coherence function at DRE (Driver's Right Ear) due to Impact at

Header Center ..................................................................................................... 10

Figure 6: FRF and Coherence function at PLE (Passenger's Left Ear) due to Impact at

Header Center ...................................................................................................... 11

Figure 7: FRF and Coherence function at DRE (Driver's Right Ear) due to Impact at Apillar Top.

Figure 8: FRF and Coherence function at PLE (Passenger's Right Ear) due to Impact at A-pillar Top 12

Figure 9: Test Points (Yellow) on the FE model for the Pre-Test Analysis ..................... 13

Figure 10: Isometric View of the Wire Frame diagram for the Test ............................... 14

Figure 11: Different Views of the Wire Frame diagram for the Test ............................... 14

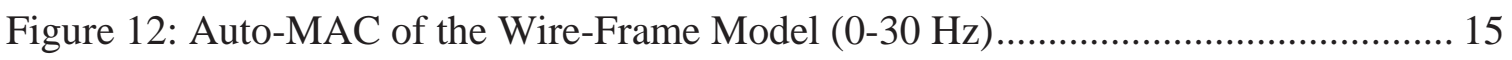

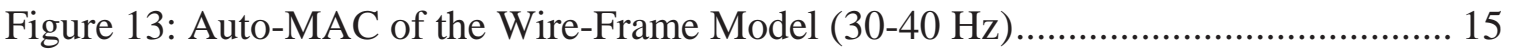

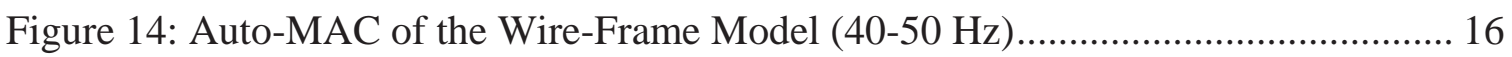

Figure 15: Initial Excitation Locations for Full Structural Modal Test............................. 16

Figure 16: Different Excitation Locations tested for new excitation locations-1 ............. 17

Figure 17: Different Excitation Locations tested for new excitation locations-2 ............. 17

Figure 18: New Excitation Locations for Full Structural Modal Test ............................. 18

Figure 19: Location of Front Shaker (Input 505)....................................................... 18

Figure 20: Zoomed view of Front Shaker (Input 505)................................................. 19

Figure 21: Location of Rear Shaker (Input 502).......................................................... 19

Figure 22: Zoomed View of Rear Shaker (Input 502) ................................................... 20

Figure 23: Experimental Setup for Full Structural Modal Test ......................................... 20

Figure 24: Passenger side view of the points measured on the vehicle ............................ 21

Figure 25: Driver side view of the points measured on the Vehicle................................ 22

Figure 26: Overlay of Drove Point FRF for the Rear Shaker .......................................... 22

Figure 27: Overlay of Drive Point FRF for the Front Shaker........................................... 23

Figure 28: FRF of DRE (Driver's Right Ear) due to input from the Rear Shaker............ 24

Figure 29: FRF of DRE (Driver's Right Ear) due to input from the Front Shaker........... 24

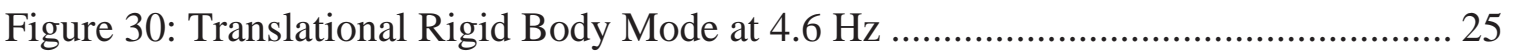

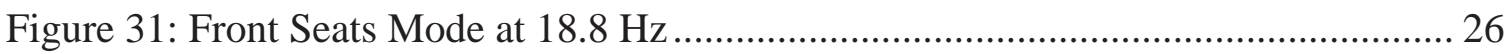

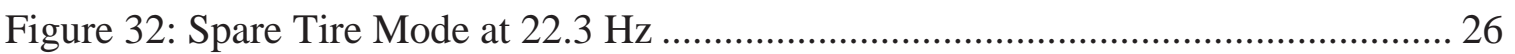

Figure 33: Front Header and Trunk Mode at $42.8 \mathrm{~Hz}$................................................. 27 
Figure 34: Structural Mode at 47.4 Hz-Rear Header and Trunk Mode .......................... 28

Figure 35: Auto-MAC of Structural Modes till $65 \mathrm{~Hz}$............................................ 28

Figure 36: Acoustic Grid in Vehicle Cabin (Front Seat) .............................................. 30

Figure 37: Acoustic Grid in Vehicle (Rear Seats and Trunk)....................................... 31

Figure 38: Acoustic Response at DRE due to Rear (Red) and Front (Blue) Shaker ........ 32

Figure 39: Acoustic FRF and Coherence below Front and Rear Header Centers due to

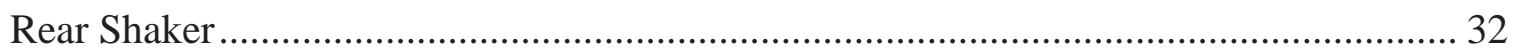

Figure 40: Acoustic FRF and Coherence below Front and Rear Header Centers due to

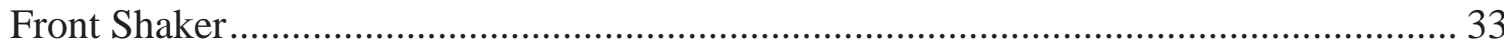

Figure 41: Acoustic FRF and Coherence at Trunk Bottom Center due to Rear (Red) and

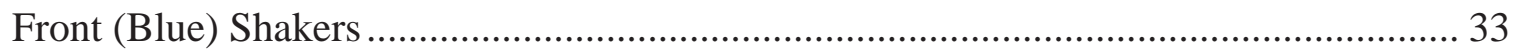

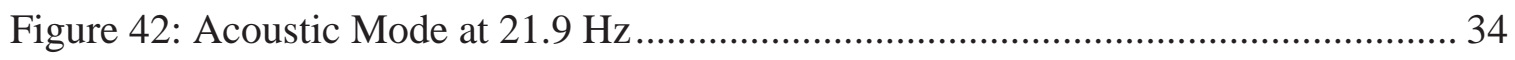

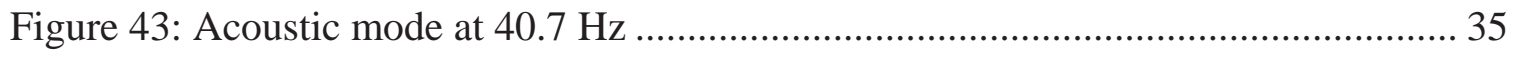

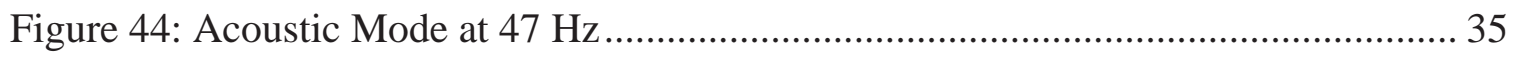

Figure 45: Acoustic Excitation (Speaker) in Car Trunk .............................................. 37

Figure 46: Acoustic Exacitation in Vehicle Cabin (Between Front Seats)....................... 38

Figure 47: Acoustic FRF at DOE and Vibration FRF at Front Header Center with

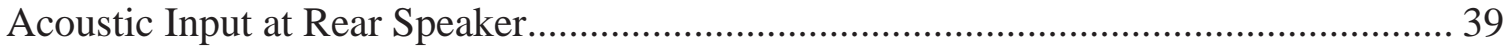

Figure 48: Acoustic FRF at DOE and Vibration FRF and Front Header Center with

Acoustic Excitation at Front Speaker .................................................................. 39

Figure 49: Acoustic FRF below Front Header Center and Vibration FRF at Front Header

Center due to Rear Speaker..................................................................................... 40

Figure 50: Acoustic FRF below Rear Header Center and Vibration FRF at Rear Header

Center due to Rear Speaker....................................................................................... 41

Figure 51: Acoustic Mode Shape at $39 \mathrm{~Hz}$ due to Speaker Inputs ................................ 41

Figure 52: Acoustic Mode Shape at $41.14 \mathrm{~Hz}$ due to Speaker Inputs ............................ 42

Figure 53: Acoustic Mode Shape at $42.7 \mathrm{~Hz}$ due to Speaker Inputs ............................... 42

Figure 54: Acoustic mode shape at $46.3 \mathrm{~Hz}$............................................................ 43

Figure 55: MAC for Acoustic DOFs with Shaker and Speaker Excitation ..................... 43

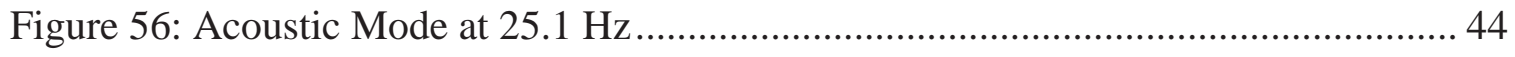

Figure 57: Chevrolet Cruze with Door Panels Removed, Clockwise: Driver Door, Front

Passenger Door, Rear Door behind Front Passenger, and Rear Driver behind Driver.... 46

Figure 58: Microphone locations in Door Cavities (Driver Side) .................................. 46

Figure 59: Microphone locations in Door Cavities (Passenger Side) .............................. 47

Figure 60: Driver Door sealed with OSB Sheets. Clockwise from top: Driver Door, Bottom View of Driver Door, Access for Speaker hole, Driver Door with no Access, and No Access Bottom View 
Figure 61: Front Passenger Door sealed with OSB Sheets. Clockwise from top: Front Passenger Door, Bottom View of Front Passenger Door, Access for Speaker hole, Front Passenger Door with no Access, and No Access Bottom View .................................... 48 Figure 62: Rear Passenger Door behind Front Passenger sealed with OSB Sheets. Clockwise from top: Rear Passenger Door, Bottom View of Rear Passenger Door, Access for Speaker hole, Rear Passenger Door with no Access, and No Access Bottom View .. 48 Figure 63: Rear Passenger Door behind Driver sealed with OSB Sheets. Clockwise from top: Rear Passenger Door, Bottom View of Rear Passenger Door, Access for Speaker hole, Rear Passenger Door with no Access, and No Access Bottom View .................... 49 Figure 64: Acoustic FRF at DRE due to Rear Shaker for different Door Configurations 49 Figure 65: Acoustic FRF at DRE due to Front Shaker for different Door Configurations

Figure 66: Acoustic Response in Driver Door Cavity due to Rear Shaker for different Door Configurations .....

Figure 67: Acoustic Response in Driver Door Cavity due to Front Shaker for different Door Configurations

Figure 68: Acoustic Response in Front Passenger Door Cavity due to Rear Shaker for different Door Configurations.

Figure 69: Acoustic Response in Front Passenger Door Cavity due to Front Shaker for different Door Configurations. 53

Figure 70: Acoustic Response in Rear Passenger Door Cavity behind Driver due to Rear Shaker for different Door Configurations 53

Figure 71: Acoustic Response in Rear Passenger Door Cavity behind Driver due to Front Shaker for different Door Configurations 54

Figure 72: Acoustic Response in Rear Passenger Door Cavity behind Front Passenger due to Rear Shaker for different Door Configurations 55 Figure 73: Acoustic Response in Rear Passenger Door Cavity behind Front Passenger due to Front Shaker for different Door Configurations ....................................................... 55

Figure 74: Location of Transducers on the Door, Door Cavity and Vehicle Cabin ......... 56 Figure 75: Structural and Acoustic Response for Driver door due to Rear Shaker .......... 57 Figure 76: Structural and Acoustic Response for Front Passenger door due to Rear Shaker

Figure 77: Structural and Acoustic Response for Rear Passenger door behind Driver due to Rear Shaker. 58

Figure 78: Structural and Acoustic Response for Rear Passenger Door behind Front Passenger due to Rear Shaker.

Figure 79: Clockwise from Top: Rear seats removed; Trunk Cavity open to Main Cavity (Perspective View); Trunk Cavity open to Main Cavity (Back View); Trunk Cavity open to Main Cavity (Side View). 


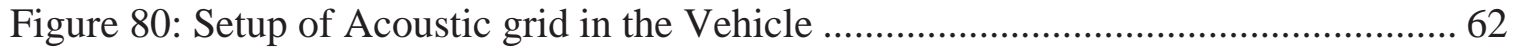

Figure 81: OSB sheet blocking Main Cavity from the Trunk Cavity ............................... 62

Figure 82: Package shelf speakers blocked by OSB sheets .......................................... 63

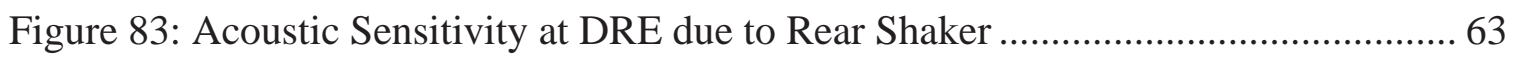

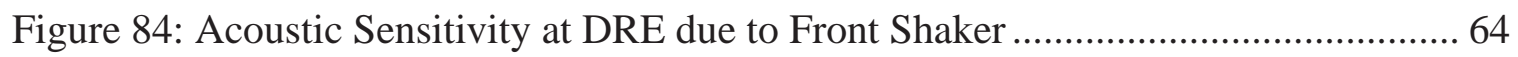

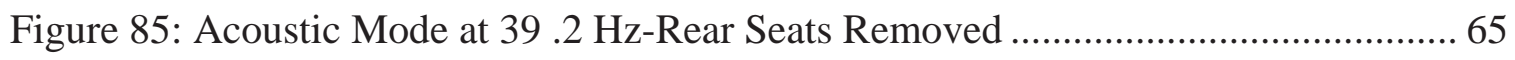

Figure 86: Acoustic Mode at 39 Hz-Trunk Cavity Blocked.......................................... 65

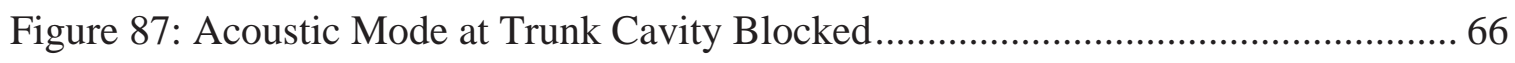

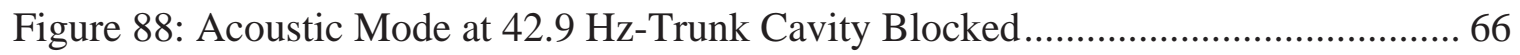

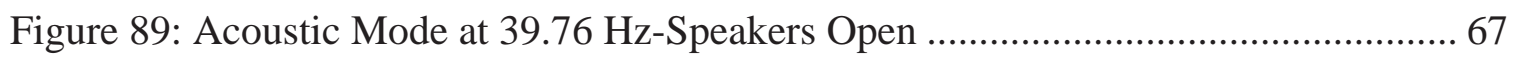

Figure 90: Acoustic Mode at 43 Hz-Package Speakers Open ......................................... 68

Figure 91: Transducers on OSB sheet for Seatback movement Testing ......................... 68

Figure 92: Transducers on GM production seats for Seatback Movement Testing ......... 69

Figure 93: Vibro-Acoustic Mode at 39 Hz (OSB-Speaker Closed) ................................ 70

Figure 94: Vibro-Acoustic Mode at 39.2 Hz (OSB-Speaker Closed) .............................. 70

Figure 95: Vibro-Acoustic Mode at $42.2 \mathrm{~Hz}$ (OSB-Speaker Closed) ............................. 71

Figure 96: Vibro-Acoustic Mode at $39 \mathrm{~Hz}$ (OSB-Speaker Open) ................................. 71

Figure 97: Vibro-Acoustic Mode at $39.3 \mathrm{~Hz}$ (OSB-Speaker Open) ............................... 72

Figure 98: Vibro-Acoustic Mode at $42.3 \mathrm{~Hz}$ (OSB-Speaker Open) ............................... 73

Figure 99: Vibro-Acoustic Mode at 39.2 Hz (GM Seats Speaker Closed)...................... 73

Figure 100: Vibro-Acoustic Mode at $40.5 \mathrm{~Hz}$ (GM Seats Speaker Closed)..................... 74

Figure 101: Vibro-Acoustic Mode at $42.5 \mathrm{~Hz}$ (GM Seats Speaker Closed)..................... 74

Figure 102: Vibro-Acoustic Mode at $39.3 \mathrm{~Hz}$ (GM Seats Speakers Open)..................... 75

Figure 103: Vibro-Acoustic Mode at $42.6 \mathrm{~Hz}$ (GM Seats Speaker Open) ...................... 76

Figure 104: Structural Model of Chevrolet Cruze .......................................................... 77

Figure 105: Acoustic Model of Chevrolet Cruze showing seats and door cavities .......... 77

Figure 106: Complete Acoustic Model of Chevrolet Cruze ........................................... 78

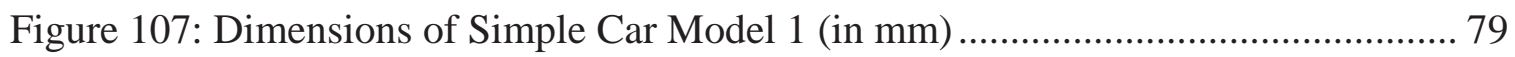

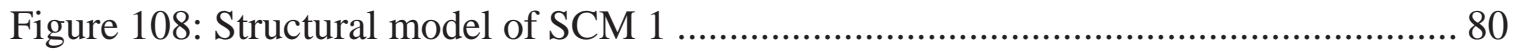

Figure 109: Cut Section of Acoustic Model for FEM …............................................ 80

Figure 110: Cut section of entire model showing Field Point Mesh and location of DRE

Figure 111: Simulation of Front Shaker and Rear Shaker input.................................. 81

Figure 112: Drive Point FRFs of the structural inputs (Front and Rear Shaker) for SCM 1

Figure 113: Acoustic Sensitivity at DRE due to structural inputs (Front and Rear Shaker)

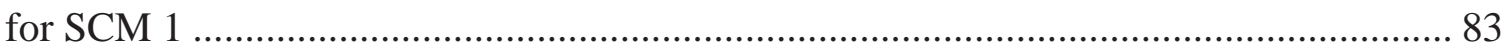

Figure 114: Structural mode and coupled mode at $55 \mathrm{~Hz}$ for BEM Case ....................... 83

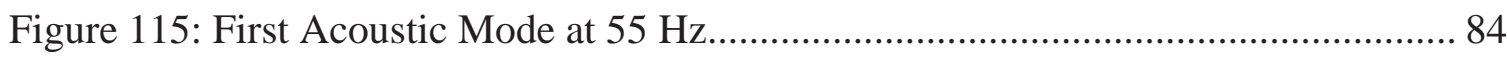


Figure 116: Dimensions of Simple Car Model 2A (in mm).

Figure 117: Left- Complete Structural Mesh of SCM2A; Right- 1-D elements in SCM 2A

Figure 118: Acoustic Mesh of SCM 2A ............................................................... 85

Figure 119: Simulation of Front Shaker and Rear Shaker input on SCM2A .................. 86 Figure 120: Drive Point FRFs of the structural inputs (Front and Rear Shaker) for SCM 2A. 86

Figure 121: Acoustic Sensitivity at DRE due to structural inputs (Front and Rear Shaker) for SCM 2A.

Figure 122: Acoustic MAC between Coupled Modes and Pure Acoustic Modes for SCM

2A. 88

Figure 123: Acoustic Mesh for SCM 2B (model without door cavities)........................ 89

Figure 124: Acoustic Sensitivity at DRE due to structural inputs (Front and Rear Shaker) for SCM 2B 90

Figure 125: Acoustic MAC between Coupled Modes and Pure Acoustic Modes for SCM

2B . 90

Figure 126: Acoustic Model for SCM 3A 91

Figure 127: Bottom view of SCM 3A showing connection between main cavity and door cavities . 91

Figure 128: Zoomed view showing connection between door cavity and main cavity ... 91 Figure 129: Acoustic Sensitivity at DRE due to front and rear shaker input for SCM 3A 92

Figure 130: Comparison of Acoustic Sensitivity at DRE for SCM 2A and SCM 3A..... 93 Figure 131: Acoustic MAC between Coupled Modes and Pure Acoustic Modes for SCM

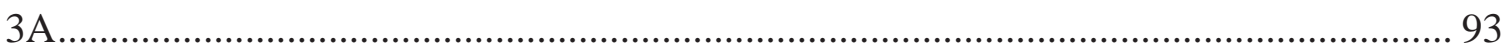

Figure 132: Acoustic Model for SCM 3B............................................................... 94 Figure 133: Acoustic Sensitivity at DRE due to front and rear shaker input for SCM 3B94 Figure 134: Acoustic MAC between Coupled Modes and Pure Acoustic Modes for SCM $3 \mathrm{~B}$ 95

Figure 135: Acoustic Sensitivity Comparison between SCM3A and SCM3B............... 95

Figure 136: Acoustic Model for SCM4A ................................................................ 96

Figure 137: Acoustic Sensitivity at DRE due to front and rear shaker input for SCM 4A 96

Figure 138: Acoustic MAC between Coupled Modes and Pure Acoustic Modes for SCM

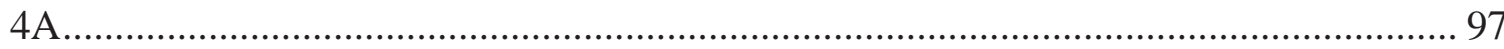

Figure 139: Acoustic Model of SCM 4B ................................................................ 98

Figure 140: Top View of SCM 4B showing the speaker openings .............................. 98

Figure 141: Acoustic Sensitivity at DRE due to front and rear shaker input for SCM 4B99

Figure 142: Spring Mass lumped parameter representation of SCM 4B ....................... 99 
Figure 143: Acoustic Sensitivity Comparison between SCM4A and SCM4B.............. 100 Figure 144: Acoustic MAC between Coupled Modes and Pure Acoustic Modes for SCM 4B. 


\section{Acknowledgements}

I would like to thank my advisor Prof. Charles Van Karsen for all the help, advice and guidance, not just in this project but towards my career overall. A large portion of the credit, of where I am today, goes to you.

I would also like to thank my project advisor, Dr. James De Clerck for coming up with creative ideas to tackle difficult issues as well as for his deep insight in the various noise and vibration concepts. Big thanks to Dr. Gregory Odegard as well for his guidance and support with finite element modeling and different FE-solvers. I am thankful to Prof. John Irwin for agreeing to be on the committee for my defense.

I am grateful to General Motors for sponsoring the project and the General Motors team, Mr. Kurt Schneider, Mr. Joseph Schudt, Mr. Robert Scheidt and Mr. Christopher Stirlen for all the help in the project.

A big shout out to my project partner Shreerang Dhabe for helping me during and after the project. You made the whole project memorable and enjoyable. I am also indebted to Aniket Parbat and Gaurav Agnihotri for their help whenever I was stuck in the project. You guys are great. Special thanks to all my friends at Michigan Tech.

I would like to thank my parents and family for their love and support throughout my stay here. Words are not enough to express my gratitude. I am also blessed to have a crazy sister, Priyanka, who helped me whenever I needed her.

I would also like to thank Manasee for adding a much needed spice in my life. Thank you for being there for me. 


\begin{abstract}
This thesis aims at understanding the vibro-acoustic behavior related to vehicle boom in passenger cars. Acoustic boom in the $40 \mathrm{~Hz}$ frequency range is studied on a 2013 Chevrolet Cruze LTZ model. Impact testing, full vehicle structural and acoustic modal tests are first performed to understand, locate and test the boom phenomenon and the fluid-structure interaction between the vehicle structure and acoustic cavity. Later, different iterations like effect of door cavities, trunk cavity and rear seats are also tested to understand their participation. LMS Test.Lab software is used for data acquisition, processing and analysis of the test results.
\end{abstract}

Finite Element Analysis (FEA) is also carried out for car shaped simple box like structures to help understand the fluid-structure interaction. Different analysis techniques such as coupled and uncoupled Boundary Element method (BEM) and Finite Element Method (FEM) are performed to understand the difference between the techniques. Iterations for effect of door cavities and rear seats are also performed for these simple car models (SCM). HyperMesh, LMS Virtual.Lab and MATLAB software packages are used for modeling, analysis and plotting of results.

With the help of this thesis, an insight in the vibro-acoustic interaction for passenger cars is gained. 


\section{Introduction}

With the fierce competition in the auto industry and the ever increasing consumer expectations, auto manufacturers are trying to achieve the goal of high performance and passenger comfort along with affordable prices. With the efforts of increasing fuel economy in the vehicle, cavity boom is one of the side effects, as the vehicle structure becomes lighter. This report describes the work done (Finite Element Analysis as well as testing) on Chevrolet Cruze LTZ to understand the acoustic cavity boom generation phenomenon.

The testing of the Cruze was carried out without the powertrain and suspension components on the vehicle. Structural measurements were performed for the entire vehicle. A total of 286 points were measured in all the three global directions. The vehicle was structurally excited with the help of two $50 \mathrm{lb}$. shakers. One shaker was located at the back of the vehicle (below the trunk) while the other was located on the frame rail below the front passenger. LMS Test.Lab software was used to collect as well as process the data. PolyMAX curve-fitting algorithm was used to find and process the different mode shapes of the vehicle. Acoustic measurements were also performed on the vehicle. A 52 microphone array was placed in the vehicle cabin and the trunk. Acoustic pressure was measured due to the structural input. The vehicle was acoustically excited as well. Excitation was with two speakers, one in the vehicle cabin, between the front seats and second in the trunk. Acoustic mode shapes were computed in addition to the structural modes. Tests were also performed on the vehicle to understand the effect of door cavities, rear seats and seat back movement.

Along with the physical testing, simple car shaped boxes were also modeled to understand the vibro-acoustic phenomenon. Modeling was performed in HyperMesh software while the analysis and post processing was done in LMS Virtual.Lab and MATLAB respectively. Similar to the physical testing, models were built to understand the effect of door cavities and rear seats. 


\section{Literature Review}

Phil Shorter [1] classifies vehicle interior sound into air-borne and structure-borne sound. Further classification leads to tire patch noise, road noise, powertrain noise and wind noise. Air-borne noise consists mainly of 'non resonant' transmission from the vehicle body structure to the cabin interior while structure-borne noise consist of 'non resonant and resonant' transmission from vehicle structure to the interior. With regards to the acoustic boom, it is mainly a structure-borne sound which is a result of road/tire inputs or also due to the vehicle powertrain at certain speeds or rpm. Hardware testing was performed on a 2013 Chevrolet Cruze LTZ in this project to understand the acoustic boom present at $40 \mathrm{~Hz}$.

The need for vibro-acoustic simulation and its different techniques are discussed by Dhandole and Modak [2], Sol and Van Herpe [3] and Tsuji et al [4]. Due to the highly competitive automotive market today and the ever mounting pressure on automotive makers to increase gas mileage, today's vehicles are lighter than before. Manufacturers have started using aluminum and different composites for the body instead of regular steel. However, this has adverse impact on the cabin interior sound levels, as the vehicle become lighter and in some cases more compliant. The customer has become sensitized to noise issues and expects the best acoustic comfort possible.

Dhandole and Modak [2] mention that the strength of the vibro-acoustic coupling in a structure depends on the geometry of the structure, material properties of the structure as well as the acoustic cavity and also on the frequencies of the dynamic excitations.

The software used for vibro-acoustic simulations in this project is LMS Virtual.Lab. According to LMS theory by Desmet and Sas [5], the vibro-acoustic interaction can be classified into uncoupled and coupled systems. A one way coupling, either from the structure to the fluid or from the fluid to the structure, exists in uncoupled systems. A tank made of flexible walls containing a dense liquid is an example of a one way coupling. In a coupled system, the fluid structure interaction is much more complex and is present both ways. Figure 1 [6] shows the schematic diagram of the fluid structure interaction in coupled systems. It is a closed loop system. The structural disturbances will excite the fluid which in turn would again excite the structure. The vehicle boom issue is an interior noise problem as there is no sound radiation outside towards infinity.

Cherng et al [7] discusses about vehicle cavity resonances as well as the effect of car seats and their placements. 
A simple analytical equation to compute cavity resonances is also mentioned which is shown in equation (1)

$\omega=\frac{c}{2} \sqrt{\left(\frac{l}{L_{x}}\right)^{2}+\left(\frac{m}{M_{y}}\right)^{2}+\left(\frac{n}{N_{z}}\right)^{2}}$ radians

Where $L_{x}, L_{y}$ and $L_{z}$ are the length, breadth and height of the acoustic cavity and $1, m, n$ are the mode number in the $\mathrm{X}, \mathrm{Y}$ and $\mathrm{Z}$ direction respectively.

\section{Vibration generates 'sound'}

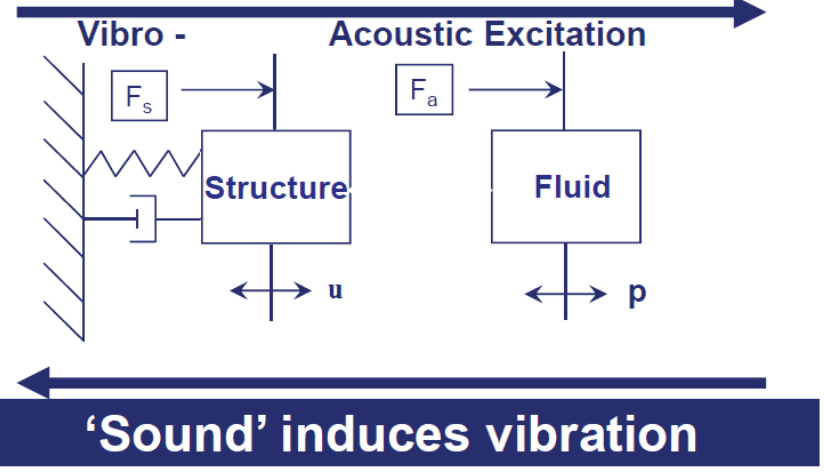

Figure 1: Fluid Structure Interaction in Coupled Systems

Cherng et al [7] also discuss about the effect of seats to the cavity modes. There is a significant reduction in the natural frequencies of the cavity as a result of addition of seats. Also, the seats act as acoustic absorbers and reduce the acoustic sensitivity at a point in the cavity.

Indirect variation BEM and FEM methods are used in this thesis for solving the models. The Boundary Element method is introduced by Desmet and Sas [5] and discussed in detail by Desmet [8]. The Boundary Element method is a two-step approach. In the first step, the values of boundary variables are determined. In the second step, the values of the field variables at any point in the domain can be found out based on the boundary variables. The equation for coupled FE/ direct BE is shown in equation (2).

$$
\left[\begin{array}{cc}
K_{s}+j \omega C_{s}-\omega^{2} M_{s} & L_{c} \\
\rho_{o} \omega^{2} B(\omega) \cdot T & A(\omega)
\end{array}\right]\left\{\begin{array}{l}
w_{i} \\
p_{i}
\end{array}\right\}=\left\{\begin{array}{l}
F_{s i} \\
F_{a i}
\end{array}\right\}
$$

Where,

$\mathrm{M}_{\mathrm{s}}$ is the structural mass matrix

$\mathrm{K}_{\mathrm{s}}$ is the structural stiffness matrix

$\mathrm{C}_{\mathrm{s}}$ is the structural damping matrix 
$\rho_{\mathrm{o}}$ is the density of the fluid

$\mathrm{L}_{\mathrm{c}}$ is the acoustic pressure loading on the elastic structure

$\mathrm{w}_{\mathrm{i}}$ is the displacement of the nodes on the structural mesh

$p_{i}$ is the pressure on the acoustic mesh

$\mathrm{F}_{\mathrm{si}}$ is the force acting on the structural mesh

$F_{a i}$ is the force acting on the acoustic mesh

$\mathrm{A}(\omega)$ and $\mathrm{B}(\omega)$ are non-symmetric full populated influence matrices

The influence matrices are related by equation (3)

$[A] .\left\{p_{i}\right\}=j \rho_{o} \omega[B] .\left\{v_{n i}\right\}$

$\mathrm{V}_{\mathrm{ni}}$ is the velocity at the node of the acoustic mesh

In the case of coupled vibro-acoustic model for the FE approach [9], equation (4) is shown below:

$\left(\left[\begin{array}{cc}K_{S} & K_{c} \\ 0 & K_{a}\end{array}\right]+j \omega\left[\begin{array}{cc}C_{S} & 0 \\ 0 & C_{a}\end{array}\right]-\omega^{2}\left[\begin{array}{cc}M_{s} & 0 \\ -\rho_{o} K_{c}^{T} & M_{a}\end{array}\right]\right) \cdot\left\{\begin{array}{l}w_{i} \\ p_{i}\end{array}\right\}=\left\{\begin{array}{l}F_{s} \\ F_{a}\end{array}\right\}$

Where,

$K_{S}, C_{S}, M_{s}$ : Structural Stiffness, Damping and Mass Matrix respectively

$K_{a}, C_{a}, M_{a}$ : Acoustic Stiffness, Damping and Mass Matrix respectively

$w_{i}, p_{i}$ : Structural Displacement and Acoustic Pressure respectively

$F_{s}, F_{a}:$ Structural and Acoustic loading respectively

$K_{c}$ : Coupling Matrix

$-\rho_{o} K_{c}^{T}$ : Coupled Mass Matrix

The coupling matrix is shown in equation (5):

$$
K_{c}=-\sum_{e=1}^{n_{s e}}\left(\int_{\Omega_{s e}}\left(\left[N_{s}\right]^{T} \cdot\left\{n^{e}\right\} \cdot\left[N_{a}\right]\right) \cdot d \Omega\right)
$$

Where,

$N_{S}$ is the global shape function for the structural model

$N_{a}$ is the global shape function for the acoustic model

$n_{s e}$ is the number of shell elements on the coupled surface $\Omega_{s e}$

$n^{e}$ is a unit vector

The BEM approach is good for complicated geometries as the meshes can be 2-D meshes which are simple to construct. The number of elements and nodes are significantly smaller in number compared to an FE model. In the case of sound radiation problems, the BEM approach inherently satisfies the Sommerfield radiation condition. However, the disadvantages are that the matrices are fully populated and take longer to solve than $\mathrm{FE}$ models. 
FE models are sparse as well as symmetric; hence, even if they are large in number, the computations are quicker. The BEM approach yields quicker results mainly for highly complicated geometries having large sizes.

For the uncoupled vibro-acoustic approach (the structure is not excited by the acoustic fluid disturbances), the concept of ATV (Acoustic Transfer Vectors) was used in the Finite Element Method (FEM) and Boundary Element Method (BEM). Gerard et al [10], McCulloch et al [11] and Citarella et al [12] discuss about the concept and advantages of the ATV approach. ATVs are also called acoustic sensitivities or contribution vectors. They are input output relation between the structural velocities of the structural model to the acoustic pressures of the acoustic model [10]. They are dependent on the surface geometry, acoustic properties of the model and any acoustic treatments on the surface of the acoustic model.

They are independent of the loading present on the structural model and hence can be calculated if the acoustic model is present and the frequency range is known. The ATV approach is a two-step approach similar to the variational Indirect Boundary Element Method (IBEM). In the first step the transfer vectors from the structural nodes of the radiating surface are calculated and in the second step, the acoustic response at a certain field point (e.g. Driver's Right Ear) is calculated for the different loading conditions. ATVs are calculated at discrete frequencies. They are smooth functions and hence, the response at intermediate frequencies can be interpolated. This improves the computational time for solving the models. ATVs are usually applicable for one way coupling only, which makes them apt for usage for the uncoupled approach. The relationship between ATVs, acoustic pressure and structural vibrations is given in equation (6)

$p(\omega)=\{\operatorname{ATV}(\omega)\}^{T}\left\{v_{n}(\omega)\right\}$

The above mentioned methods were used to model and solve the finite element models to understand the fluid-structure interactions.

For testing of the physical structure, a Multiple Input Multiple Output test was performed [13], [14]. This type of test ensures that the structure is evenly excited. As there are multiple inputs, large excitation levels are not necessary. The inputs are completely uncorrelated thus ensuring two separate principal components in the system. The structure was mounted on air bags in order to simulate a free- free boundary condition. 


\section{Tests Performed on the Chevrolet Cruze LTZ}

The list below enumerates the different tests performed on the Chevy Cruze with a brief description for each:

\subsection{Impact Hammer Test}

While trying to understand the behavior of the structure and the boom phenomenon, it was found that the front header had a good contribution to the boom at DRE. This was a very small test to find out the contribution of the front header to the acoustic boom.

\subsection{Full Structural Modal Test}

Data from 286 points (3 directions) was acquired during this test. The structure was mounted on air bags so as to achieve a free-free condition. The structure was excited with two $100 \mathrm{lb}$. shakers. These air bags were kept in inflated condition till the entire data was collected.

\subsection{Full Acoustic Modal Test - Structural Input}

Pressure from 52 data points was acquired for this test. An acoustic grid was prepared for the passenger cabin as well as the trunk. Pressure from 12 data points was acquired in the trunk and from 40 data points in the passenger cabin. In total, 52 data points were used. The two shakers that were used for structural modal test were used for this test as well.

\subsection{Full Acoustic Modal Test - Acoustic Input}

Two speakers, one in the passenger cabin and one in the trunk were used to excite the structure. To find the vibro-acoustic coupling, structural measurements were also performed at the front and rear headers of the car. The same acoustic grid which was used in the full acoustic modal test (with structural input) was used in this test.

\subsection{Full Acoustic Modal Test - Structural Input: Door Cavity Testing}

The door cavity testing was done under many configurations. This test was performed to test the theory that the acoustic cavity becomes longer due to the door cavities and that the door cavities act like Helmholtz Resonators. The door cavity testing was performed with two microphones each door cavity (total eight microphones for four doors).

The testing was performed with the original door panels removed, with OSB (Oriented Strand Boards) having an opening for the speaker and the final testing with speaker opening closed. Different types of acoustic materials were used to seal the door cavity from the main cavity. 


\subsection{Full Acoustic Modal Test- Structural Input: Effect of Rear Seats and Package Shelf Speakers}

This test was performed to understand the acoustic behavior of the rear seats and to understand the transmission of sound pressure from the trunk to the passenger cavity and vice-versa. Structural excitation from the two $100 \mathrm{lb}$. shakers was given. Testing was performed with the rear seats completely removed and with OSB (Oriented Strand Board) sheets covering the passenger cavity and trunk cavity. During these tests, the effect of rear speakers was also attempted to be understood by covering the speakers on the package shelf with small OSB sheets.

\subsection{Full Acoustic Modal Test - Structural Input: Seatback Movement Testing}

It was done to understand the movement of the car seats as well as the OSB sheets when the structure was excited with the help of the shakers. The movement of the seats would throw light on the pressure created in the passenger cabin as well as in the trunk. It would also explain the vibro-acoustic coupling and whether the structure drives the fluid inside or if it's the other way around. 


\section{Impact Hammer Test}

\subsection{Purpose}

The Impact Hammer Test was the first test that was performed on the Chevrolet Cruze LTZ. The objective of this test was to understand the vibro-acoustic coupling present in the structure.

\subsection{Description}

The structure was excited at the front header center as well as the top of the A-pillar. The acoustic response was measured at the driver's right ear (DRE) and front passenger's left ear (PLE). Structural responses were measured at the same locations where the impact was made; Figure 2 shows the impact locations on the Cruze while Figure 3 shows the acoustic response locations.

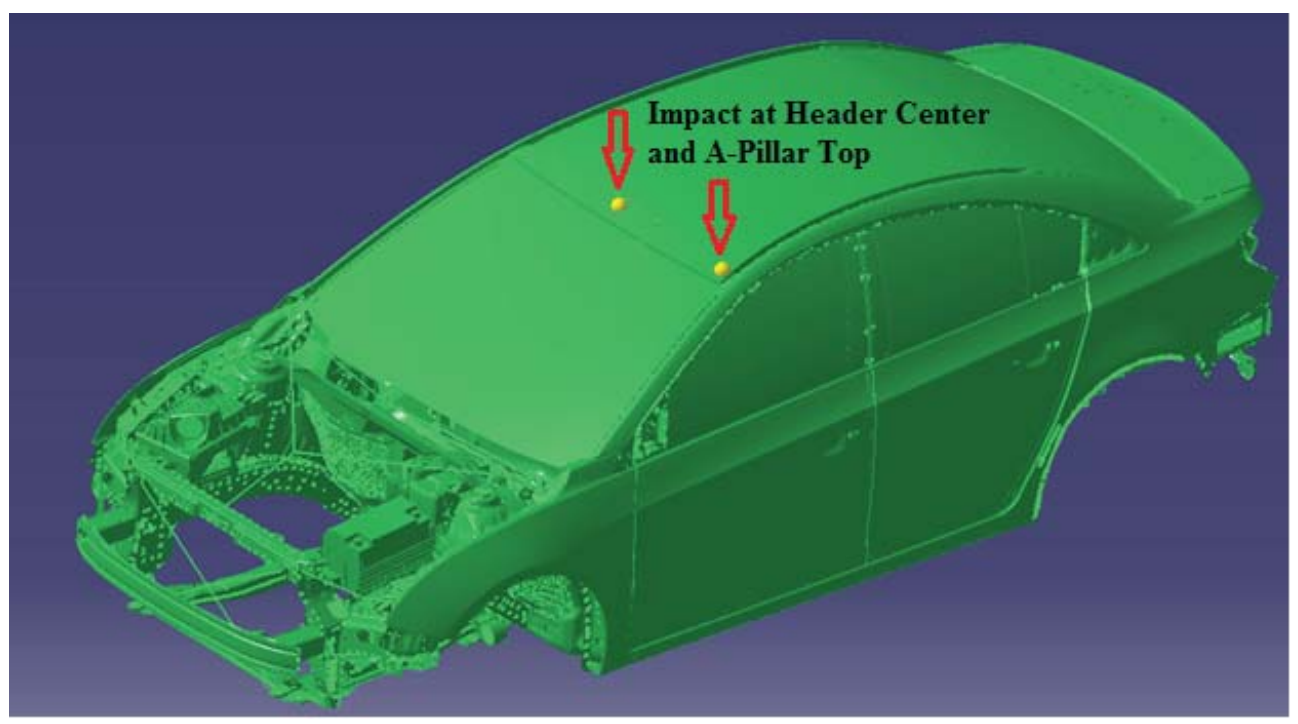

Figure 2: Impact Locations for Impact Hammer Testing 


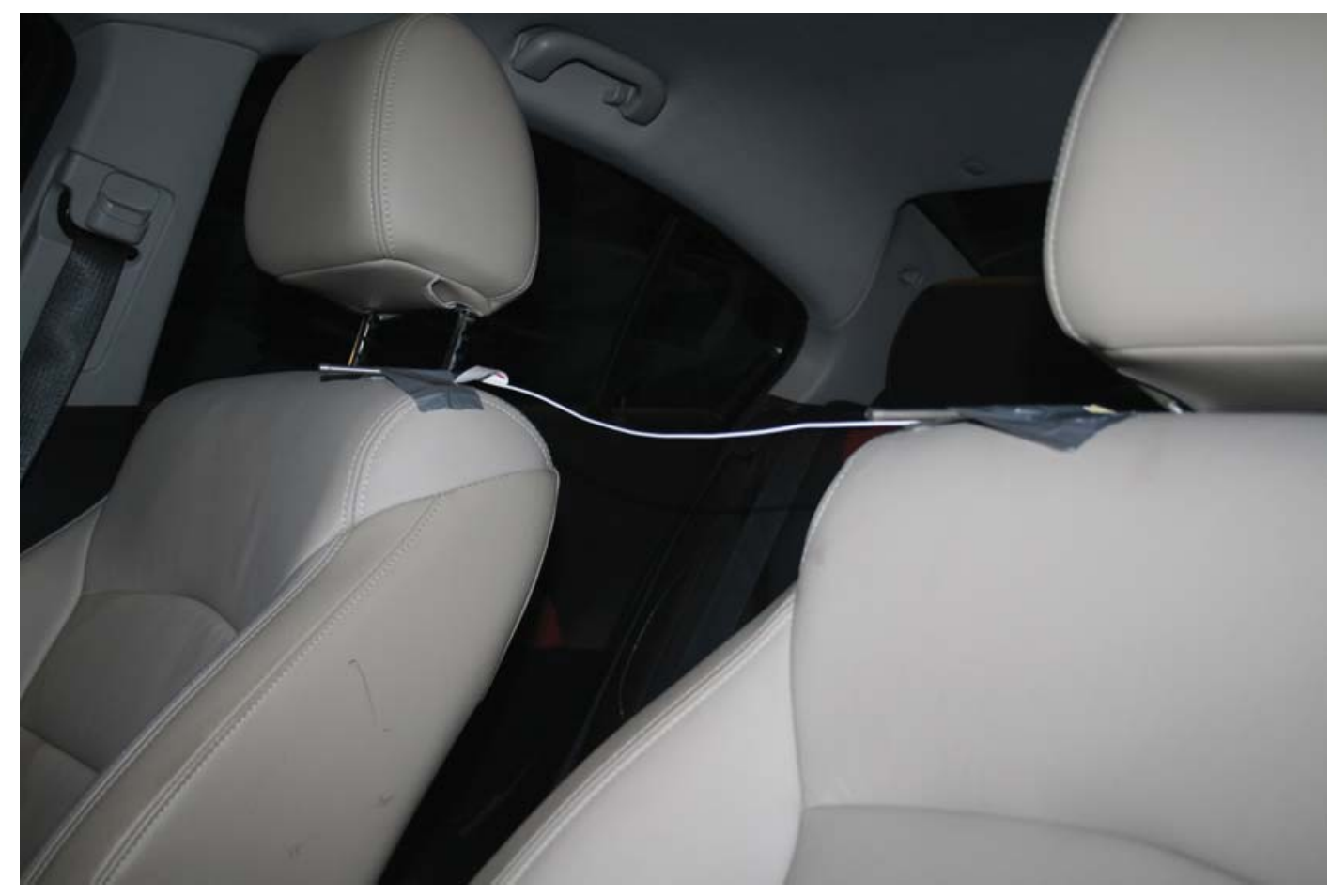

Figure 3: Acoustic Response Locations for Impact Hammer Testing

Figure 4 shows the zoomed view structural responses measured during the test.

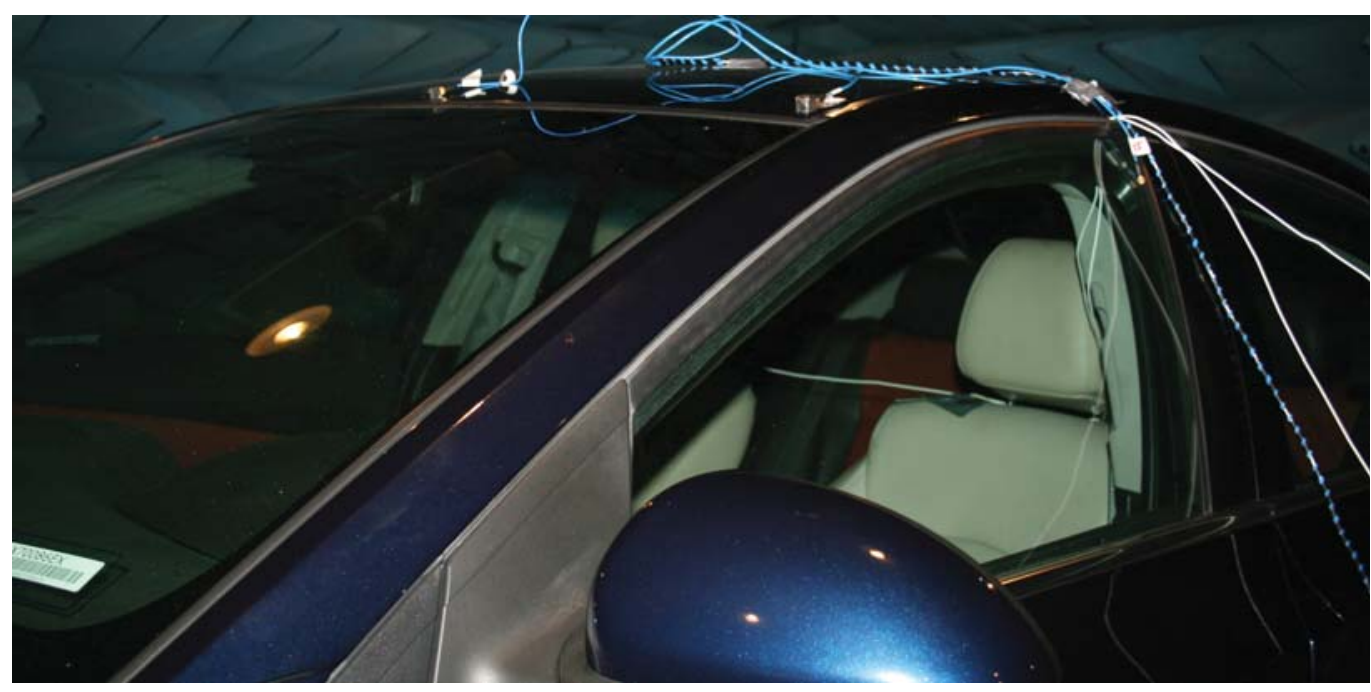

Figure 4: Zoomed View of Structural Response Locations (Header Center and A Pillar Top)

Figure 5 shows the Acoustic Sensitivity (FRF: Pa/N) response and Coherence at DRE due to impact at front header center. 


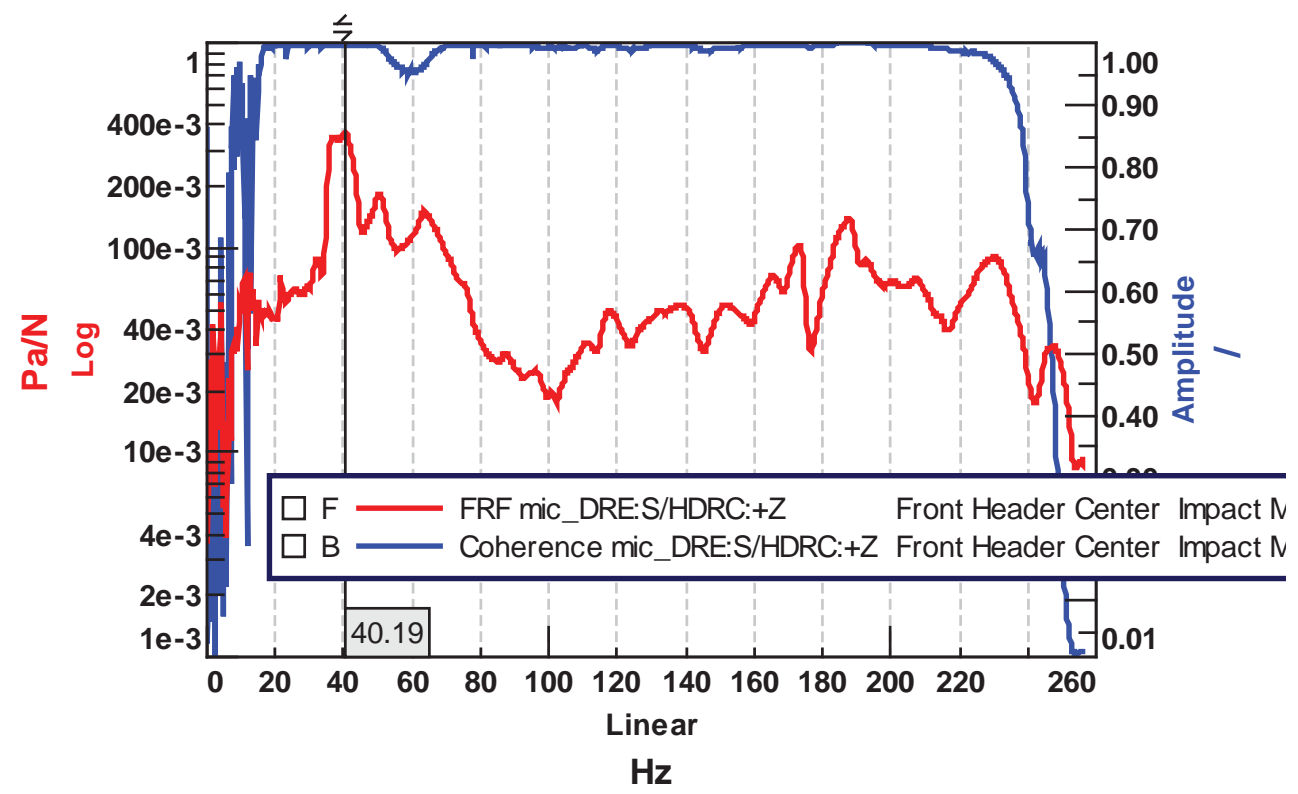

Figure 5: FRF and Coherence function at DRE (Driver's Right Ear) due to Impact at Header Center

It can be seen from Figure 5 that a high response is seen in the $40 \mathrm{~Hz}$ range. The coherence in this frequency range is unity indicating a linear response from the structure. Peaks are also seen at $50 \mathrm{~Hz}$ and $62 \mathrm{~Hz}$. However, the magnitude is half of that of the response at $40 \mathrm{~Hz}$. The FRF response is noisy and the coherence is below unity below 20 $\mathrm{Hz}$. This is because the response of the transducer (microphone) is good only between the $20-20,000 \mathrm{~Hz}$ frequency ranges. It can be seen that the coherence drops significantly after $220 \mathrm{~Hz}$. This is because of the anti-aliasing filter which starts at around $80 \%$ of the acquisition bandwidth to effectively reduce the contribution of frequency components above the desired frequency range.

Figure 6 shows the Acoustic Sensitivity at PLE (Passenger's Left Ear). The response at PLE is similar to that of DRE. High response is seen at the $40 \mathrm{~Hz}$ range indicating strong contribution from the front header. The coherence is also unity showing a linear response from the structure. Anti-resonance is seen at $176 \mathrm{~Hz}$ where the coherence also drops from unity. 


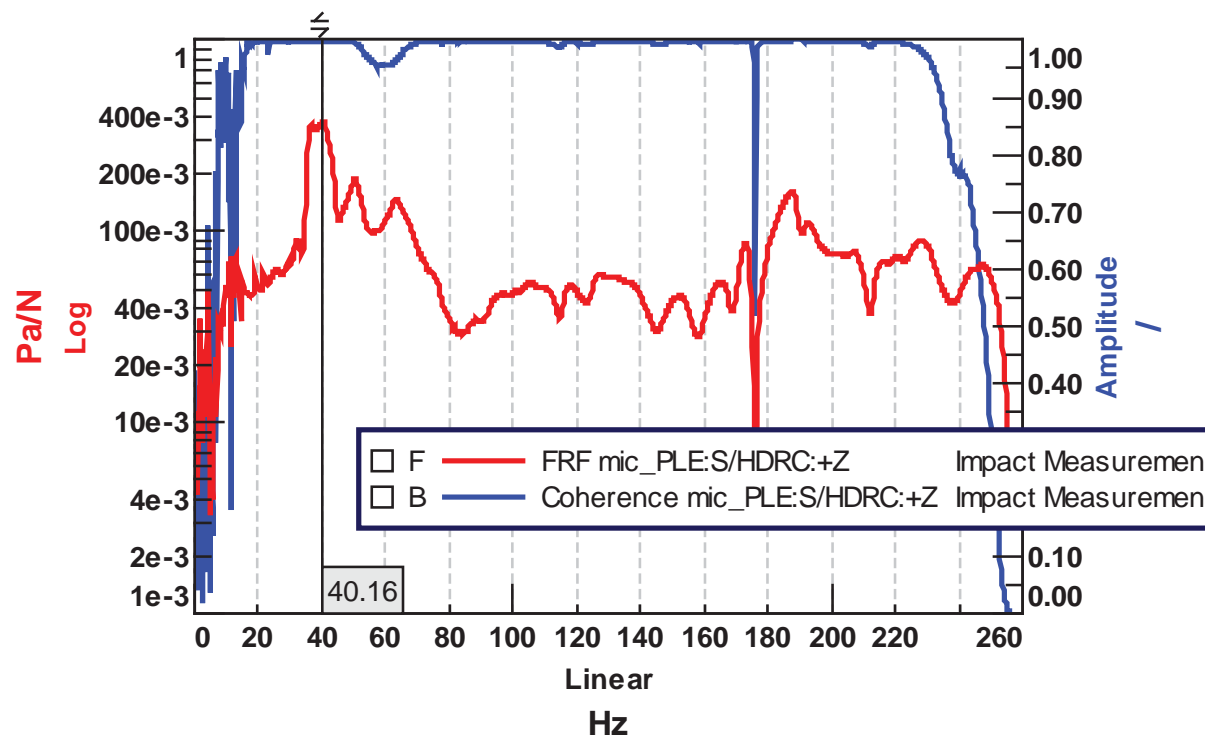

Figure 6: FRF and Coherence function at PLE (Passenger's Left Ear) due to Impact at Header Center

Figure 7 shows the acoustic sensitivity and coherence at DRE due to impact at top of Apillar. It can be seen that the response at DRE is similar to that when the impact was at the center of front header (Figure 5). The boom phenomenon can be clearly seen at the peak at $40 \mathrm{~Hz}$. However, the amplitude of the FRF is lower than due to the impact at front header center. It can be also seen that the coherence drops sharply wherever antiresonance is seen. Figure 8 shows the acoustic sensitivity response and coherence at PLE due to impact at A-Pillar top. The response at PLE clearly shows the boom phenomenon as well.

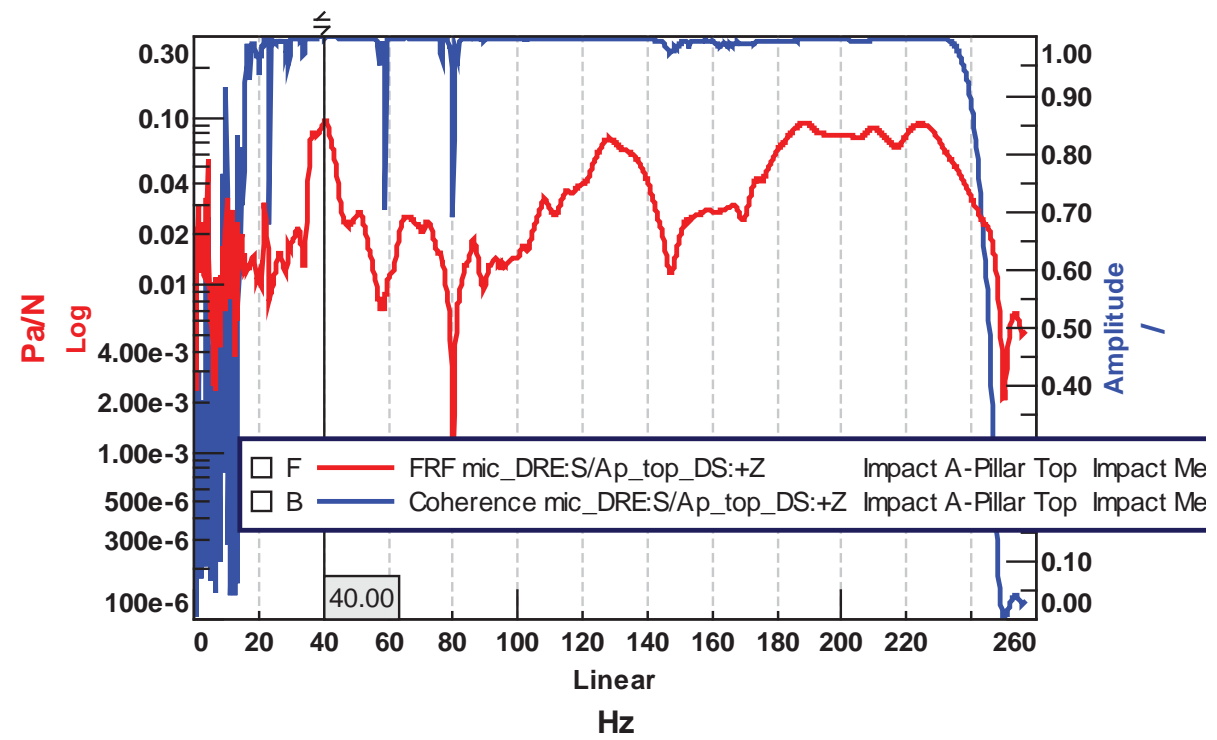

Figure 7: FRF and Coherence function at DRE (Driver's Right Ear) due to Impact at A-pillar Top 


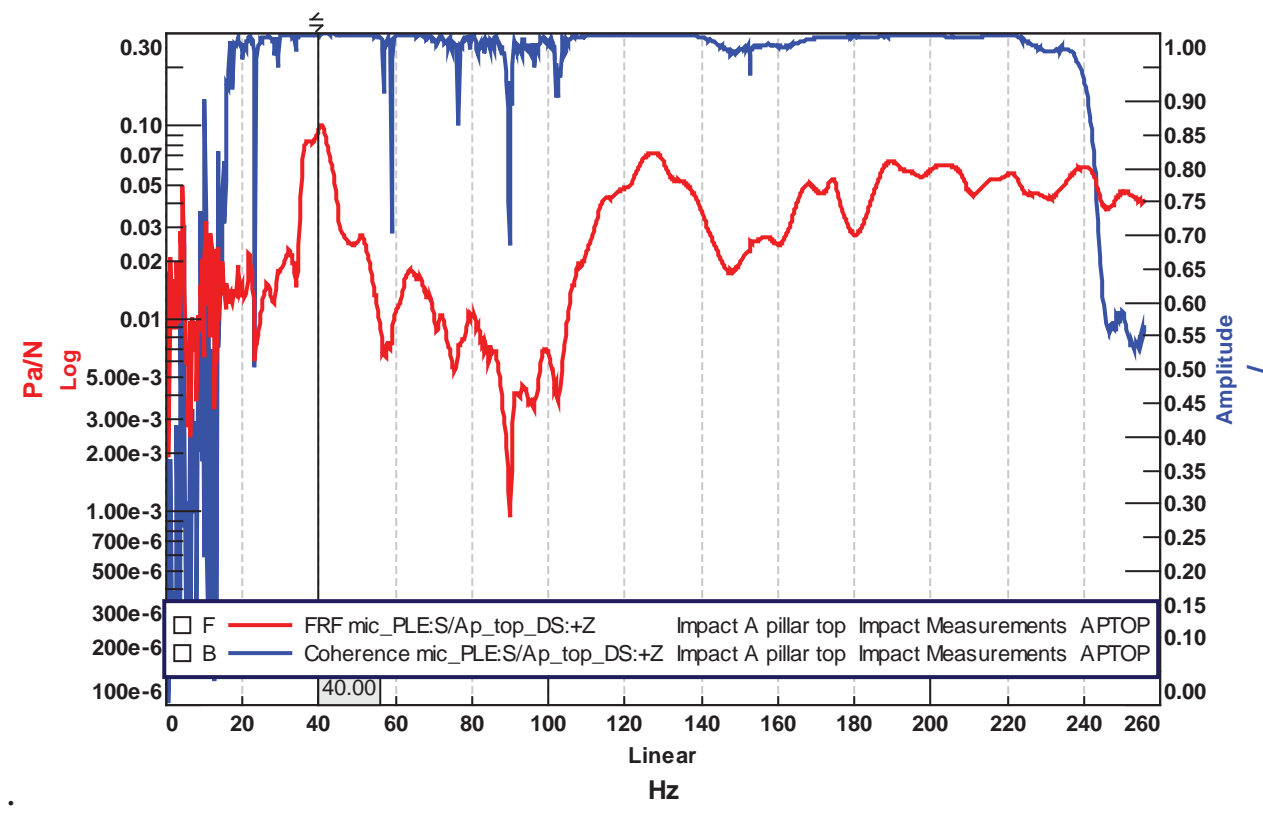

Figure 8: FRF and Coherence function at PLE (Passenger's Right Ear) due to Impact at A-pillar Top

The impact measurements were done at various other locations like the engine cradle, chassis frame and chassis floor. All the responses indicated a boom in the $40 \mathrm{~Hz}$ range (pole frequencies shifted a little). This shows that the boom phenomenon does not occur when one particular component of the vehicle is excited. High response in the $40 \mathrm{~Hz}$ range was observed at every location where the impact was done. The boom phenomenon is due to the shape and size of the cavity and also how this cavity couples with the entire structure.

\subsection{Summary of Impact Test}

This test was performed to understand the boom phenomenon and whether it is a local or a global phenomenon. The tests categorically proved that it is a global phenomenon. This test was also done to find out the optimal excitation location for the structure when the full acoustic and structural test would be performed. 


\section{Full Structural Modal Test}

\subsection{Purpose}

The full structural modal test is necessary to identify the different modes in the structure and to separate out the modes participating in the acoustic boom. This would help in understanding the motion of the structure at the boom mode.

\subsection{Description}

Before the actual testing, a Pre-Test analysis (in LMS Virtual.Lab software) was performed to ensure enough spatial density to capture all the modes in the low frequency range. Pre-Test Analysis also computes the Drive-Point Residues, which help in finding out the excitation points on the structure. If these points are chosen for excitation, there is a good probability that the entire structure would be excited uniformly so that all the modes are captured. A total of 286 points were taken on the entire structure. Figure 9 shows the points selected on the FE model for the pre-test analysis. Thus the 1.62 million nodes FE-model is reduced to just 286 points for the test. The points were taken on all the panels as well as the car frame and floor-pan so that entire motion of the vehicle was captured during data acquisition.

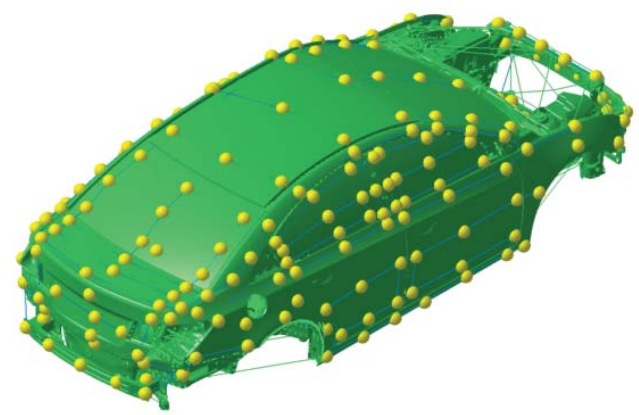

Figure 9: Test Points (Yellow) on the FE model for the Pre-Test Analysis

Figure 10 and Figure 11 show the different views of the wireframe diagram that will be used as the test geometry. 


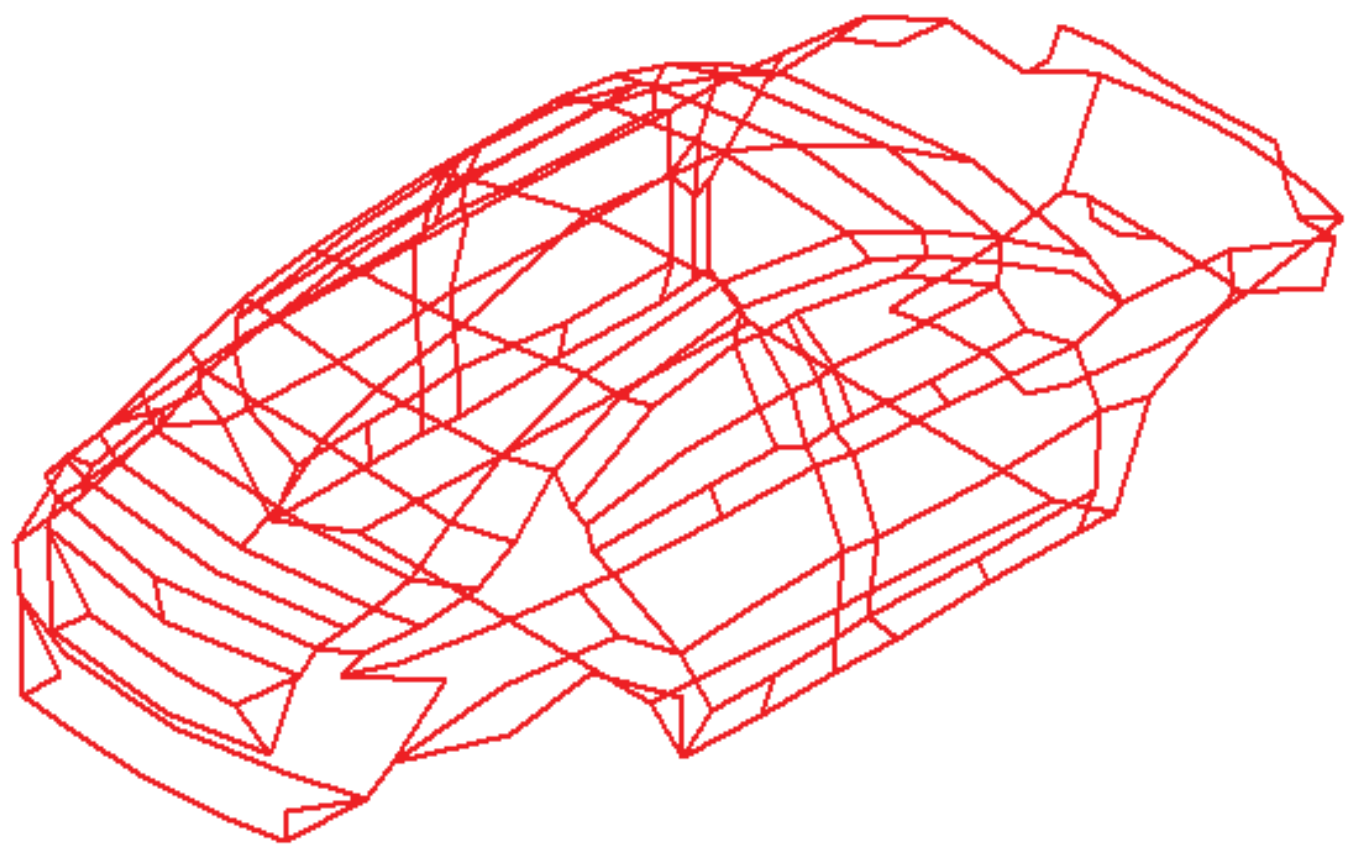

Figure 10: Isometric View of the Wire Frame diagram for the Test

From Figure 10 it can be seen that the data is measured for the front seats, steering wheel and the rear package shelf. Points are also present on the top and bottom of the spare tire. However, no points were taken from the rear seats during this test as it was decided to avoid mounting the accelerometers on the seat foam as the response would be highly nonlinear. Figure 11 shows the different views of the wireframe model.

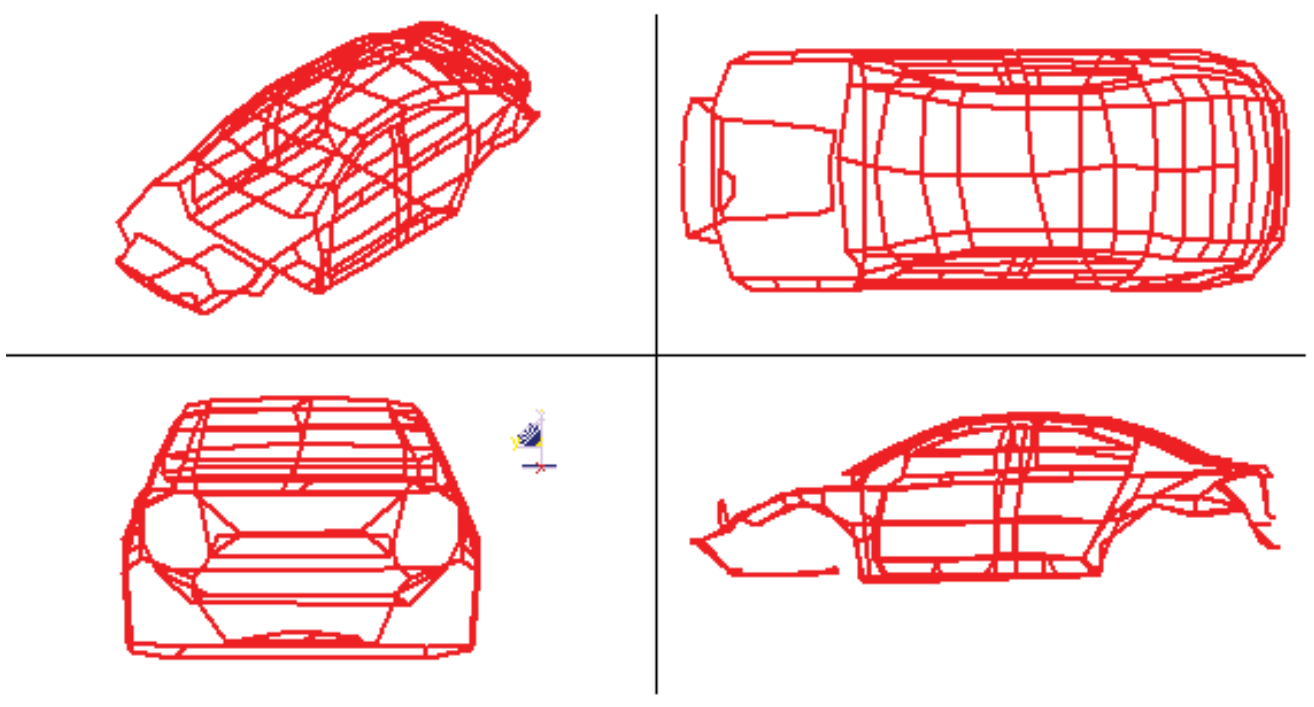

Figure 11: Different Views of the Wire Frame diagram for the Test 
Figure 12, Figure 13 and Figure 14 show the Auto-MAC (MAC of the modes with itself) for the frequency range $0-30 \mathrm{~Hz}, 30-40 \mathrm{~Hz}$, and $40-50 \mathrm{~Hz}$ respectively.

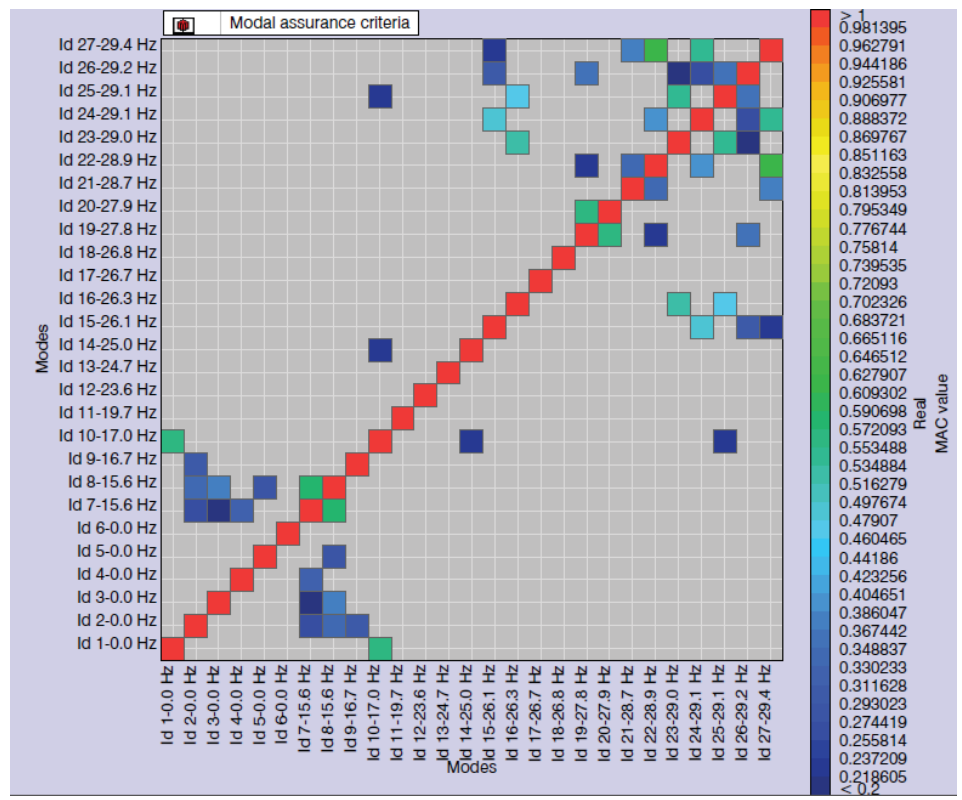

Figure 12: Auto-MAC of the Wire-Frame Model (0-30 Hz)

Ideally, the Auto MAC is a diagonal line indicating no mode shape is similar to the other. Figure 12 shows the red diagonal line; however there are other modes participating for a single mode as well. For example, the mode at $15.6 \mathrm{~Hz}$ is similar to the other $15.6 \mathrm{~Hz}$ mode (repeated root) as well the rigid body modes.

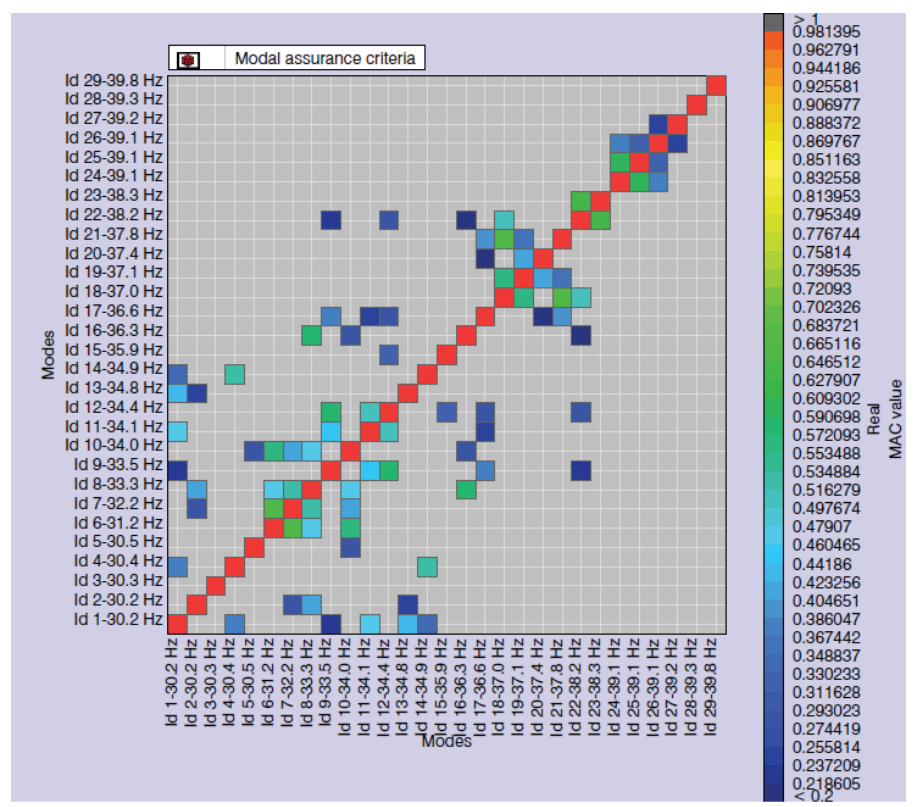

Figure 13: Auto-MAC of the Wire-Frame Model (30-40 Hz) 
Figure 13 shows the Auto-MAC for the wireframe model for the frequency range 30-40 Hz. For this range as well we see modal participation from other modes for a single mode.

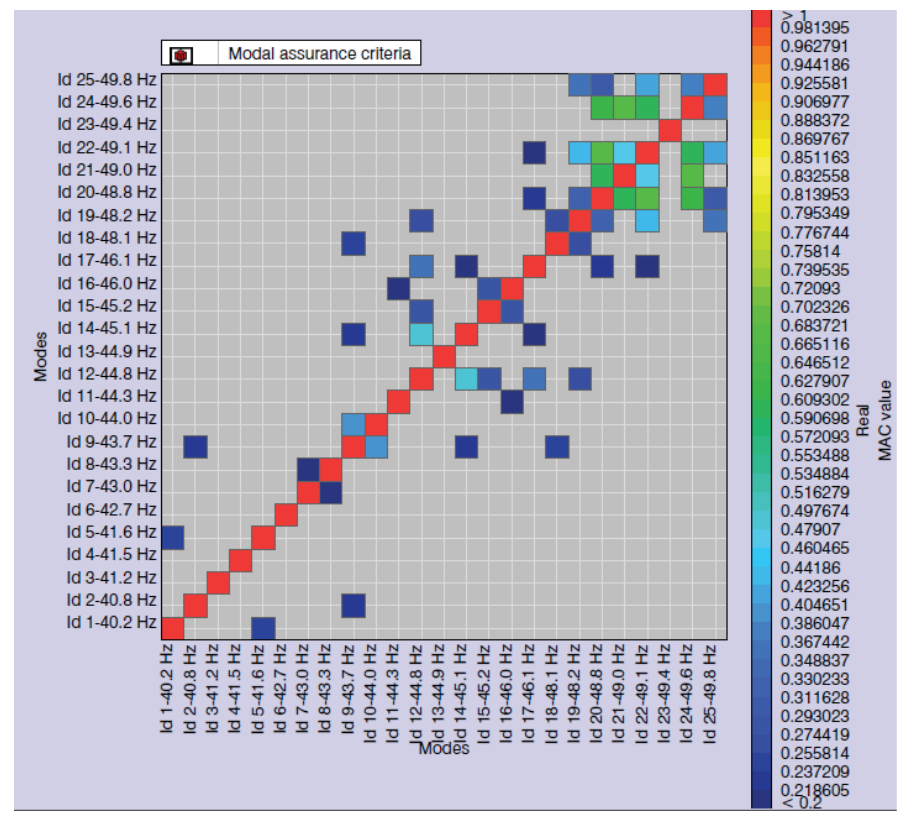

Figure 14: Auto-MAC of the Wire-Frame Model $(40-50 \mathrm{~Hz})$

Figure 14 shows the Auto-MAC for the frequency range 40-50 Hz. This MAC is also similar to the previous MACs. The modes above $48.6 \mathrm{~Hz}$ have high contribution from other modes as well.

The drive point locations were chosen as shown in Figure 15.

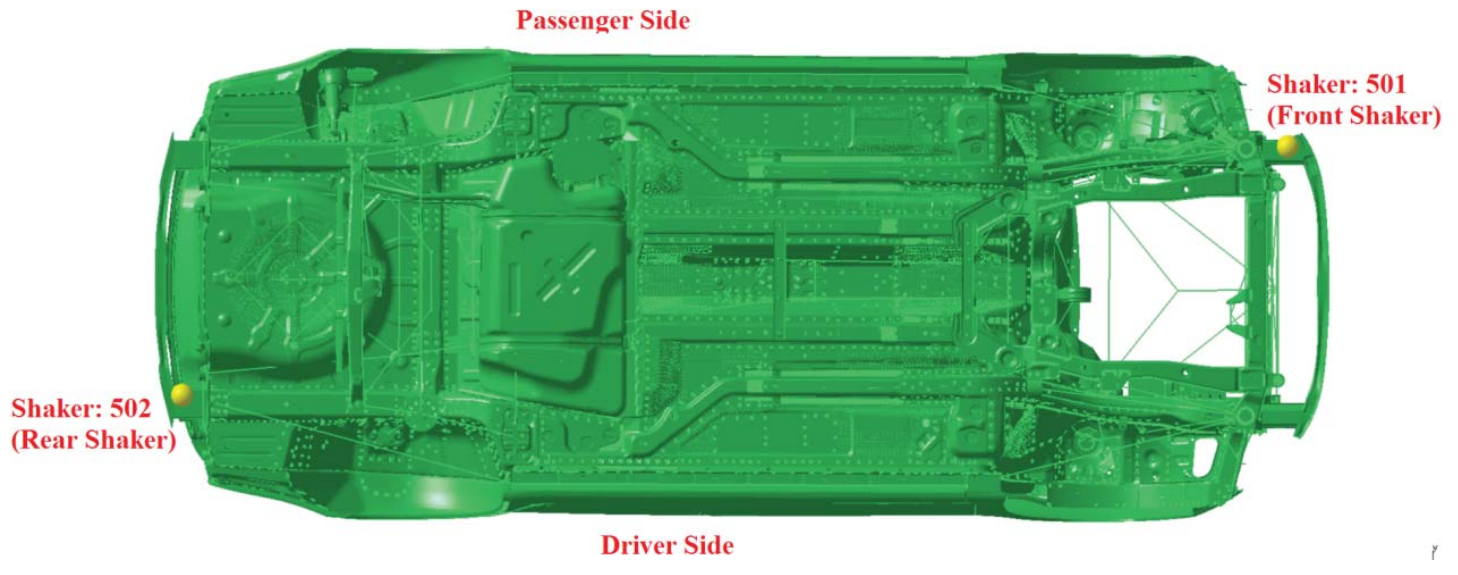

Figure 15: Initial Excitation Locations for Full Structural Modal Test 
It was found however, that the front shaker (Shaker: 501) did not excite the structure enough and the measurements were noisy.

Hence, a new excitation location was to be found out that would excite the structure well. Many different excitation locations were tried as shown in Figure 16 and Figure 17.

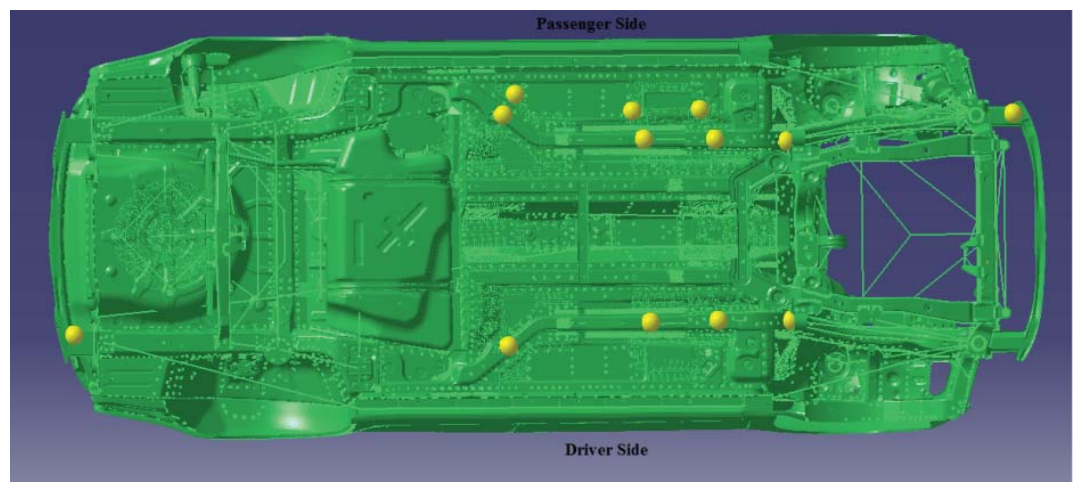

Figure 16: Different Excitation Locations tested for new excitation locations-1

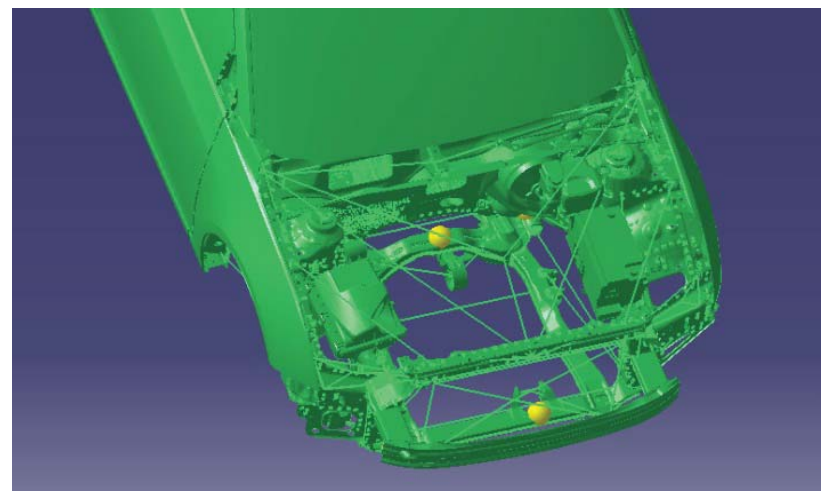

Figure 17: Different Excitation Locations tested for new excitation locations-2

The new excitation locations are shown in Figure 18. The new location was found to excite the structure well enough. The new excitation location was below the front passenger seat. It was on the frame rail of the floor pan. 


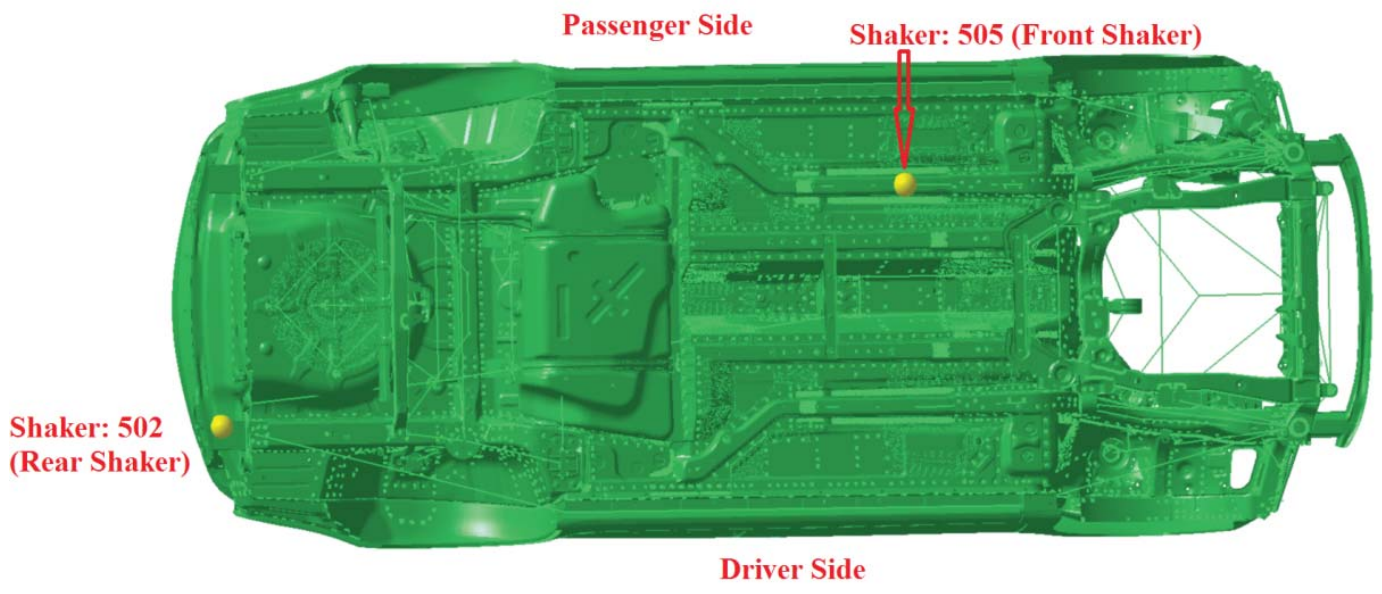

Figure 18: New Excitation Locations for Full Structural Modal Test

Figure 19 and Figure 20 show the location of the front shaker (Shaker: 505) on the actual test vehicle. Figure 20 shows the zoomed view of the front shaker. It can be seen from Figure 20 that the quill/stinger was not long. However, no errors in the measurements were observed due to the effect of the stinger. The shaker was hot-glued to the diamond plates on which it was resting, so that it would not move or rattle during the data acquisition. The glue also added some damping to absorb the vibration from the shaker thereby preventing the rattling. The load cell is attached to an aluminum piece which is super-glued on to the structure. The aluminum piece acts like a base on which the load cell will get screwed on.

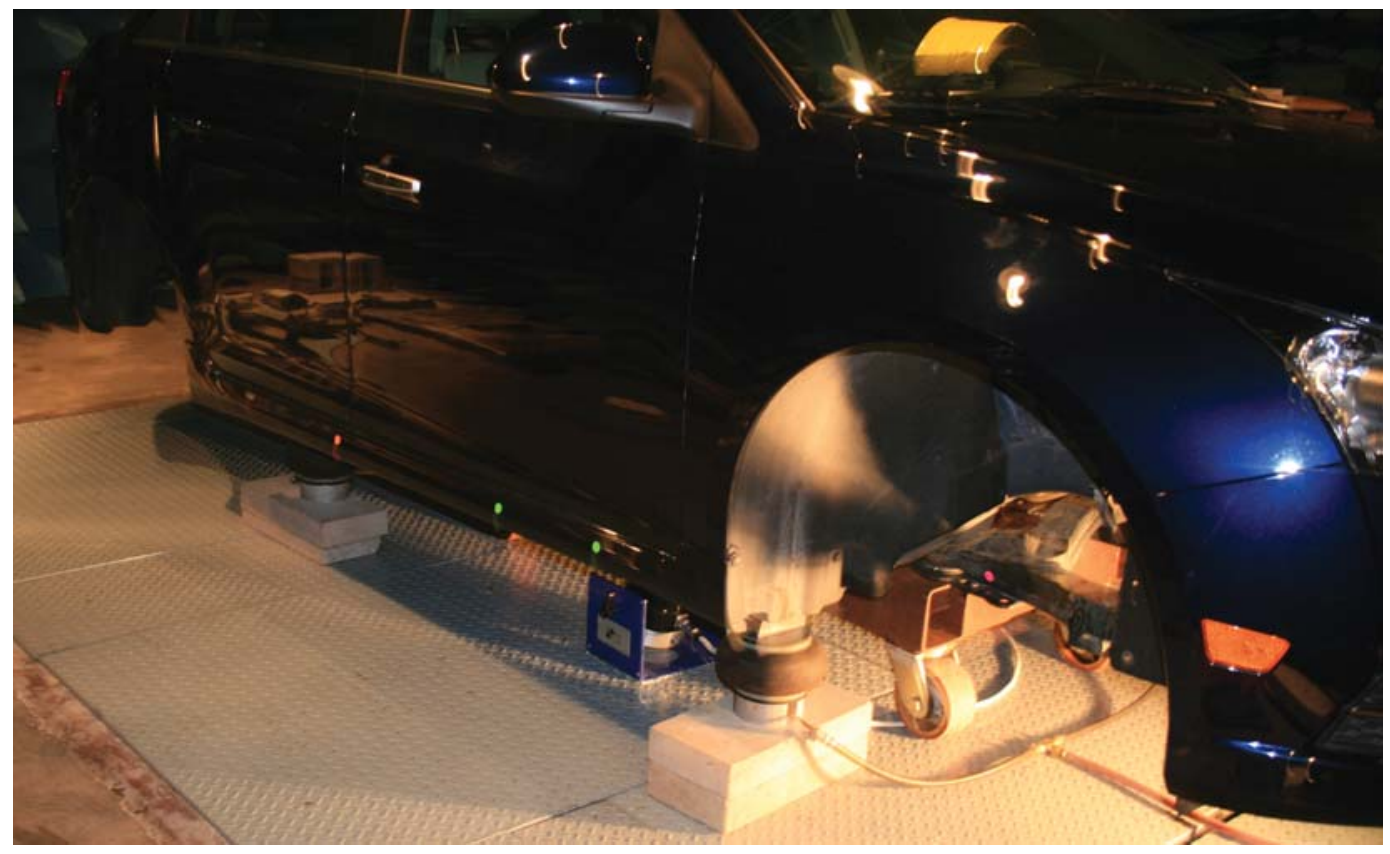

Figure 19: Location of Front Shaker (Input 505) 


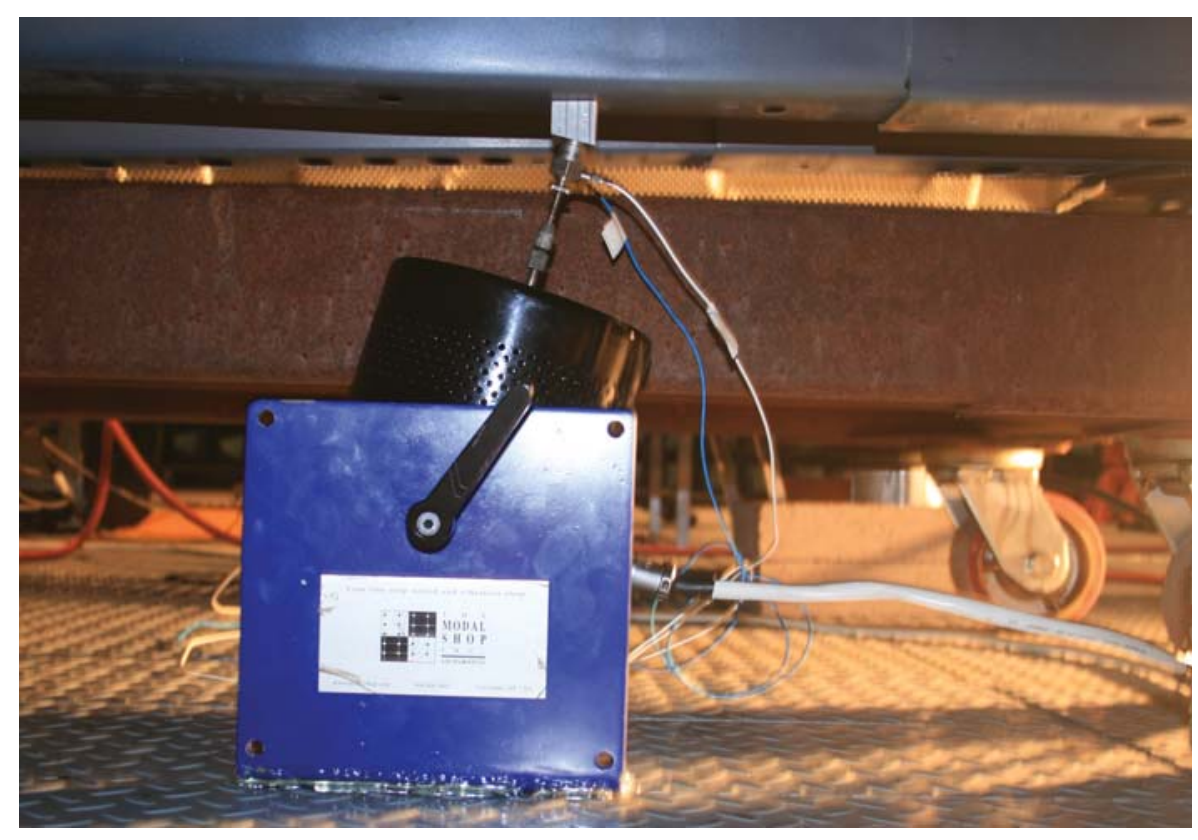

Figure 20: Zoomed view of Front Shaker (Input 505)

Figure 21 and Figure 22 show the location of the rear shaker on the vehicle. Figure 22 shows the zoomed view of the rear shaker. Putty/clay had been applied on the quill/stinger of the rear shaker as stinger resonance was observed during the preliminary tests. Hence clay was added act as a mass damper to the quill/stinger.

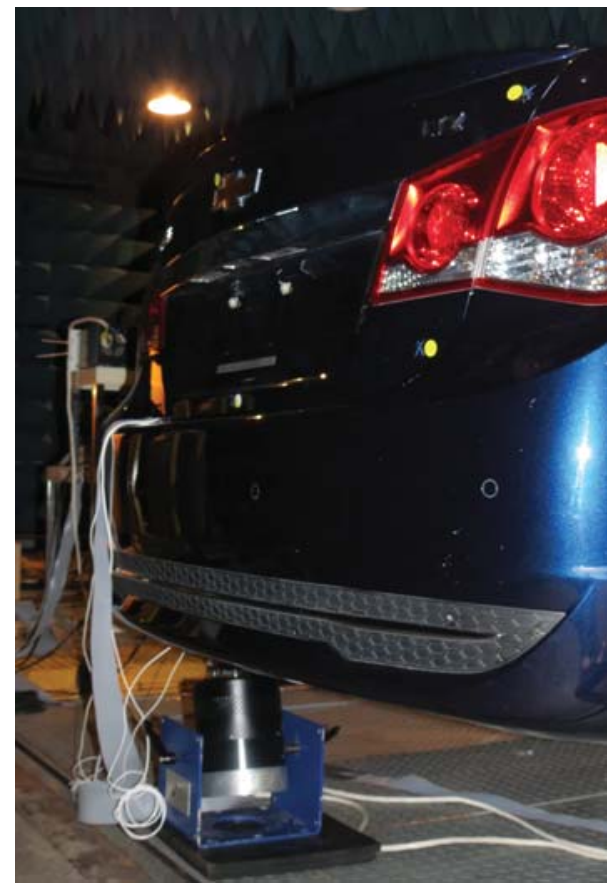

Figure 21: Location of Rear Shaker (Input 502) 


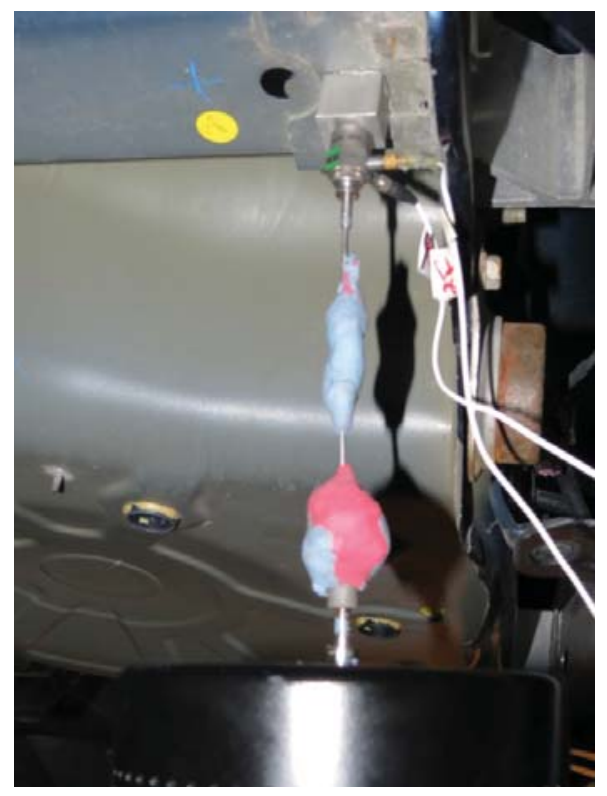

Figure 22: Zoomed View of Rear Shaker (Input 502)

Figure 23 shows the simplified experimental setup for the full structural modal test. The computer was connected to the Data Acquisition System (DAS). LMS Test.Lab 12A software was used for acquiring the data. A digital signal (burst random) for excitation of the structure was created in the software which was then sent to the digital to analog converter and then to the shakers via the amplifiers. PCB ICP transducers (Model $356 \mathrm{~A} 15)$ were mounted on the vehicle to acquire data from the structure.

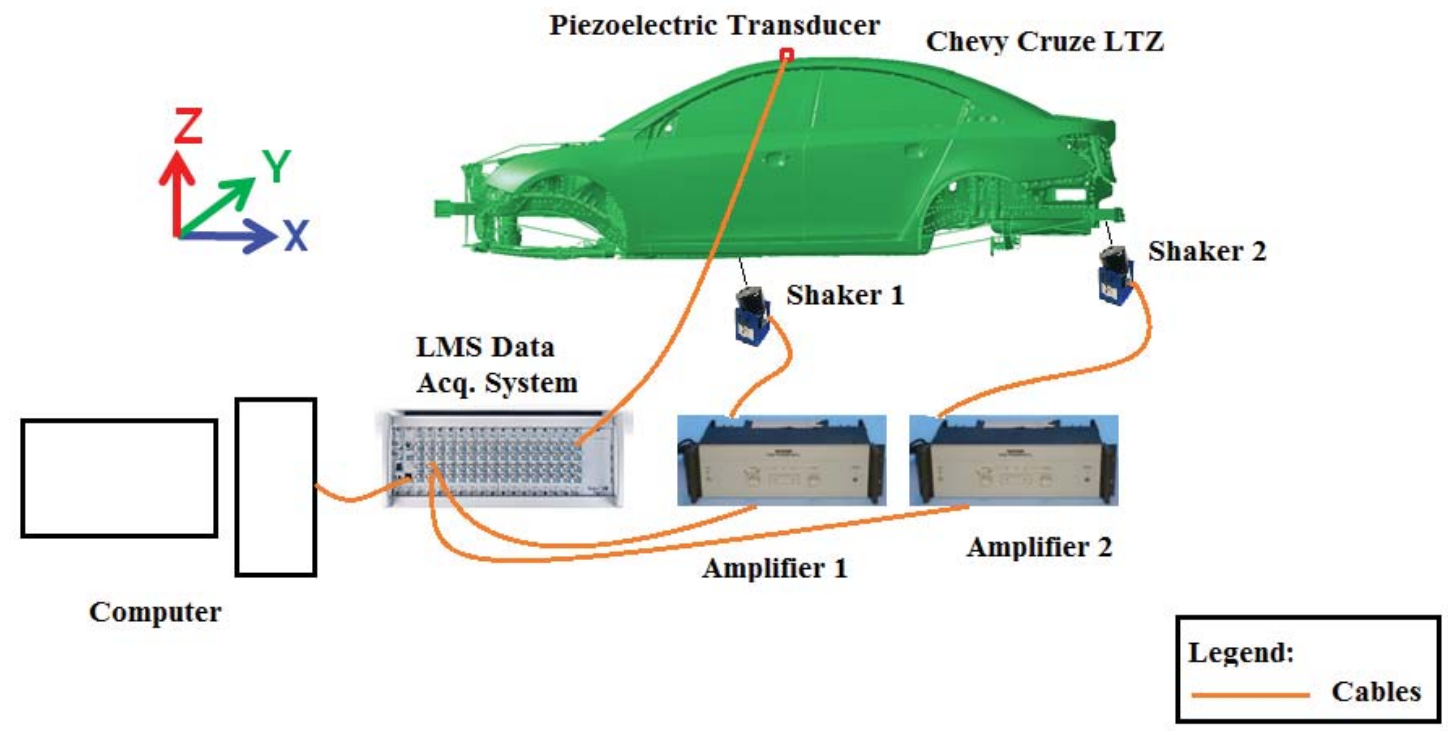

Figure 23: Experimental Setup for Full Structural Modal Test 
The data acquisition parameters used in the full structural modal test is given in Table 1 below:

Table 1: Data Acquisition Parameters used for the Full Structural Modal Test

\begin{tabular}{|c|c|}
\hline Parameter & Description \\
\hline Bandwidth & $0-256 \mathrm{~Hz}$ \\
\hline Frequency Resolution & $0.125 \mathrm{~Hz}$ \\
\hline Acquisition Time & 8 seconds \\
\hline & Type: Burst Random \\
Excitation Signal & Amplitude: 1.5 Volts \\
& Frequency Excitation: $100 \%$ of \\
& Bandwidth \\
& Burst Time: $85 \%$ \\
\hline Reference Window & Uniform \\
\hline Response Window & Uniform \\
\hline Averages & 25 \\
\hline FRF Estimation Method & H1 \\
\hline
\end{tabular}

Figure 24 and Figure 25 show the passenger side view and driver side view respectively of the different points measured on the vehicle.

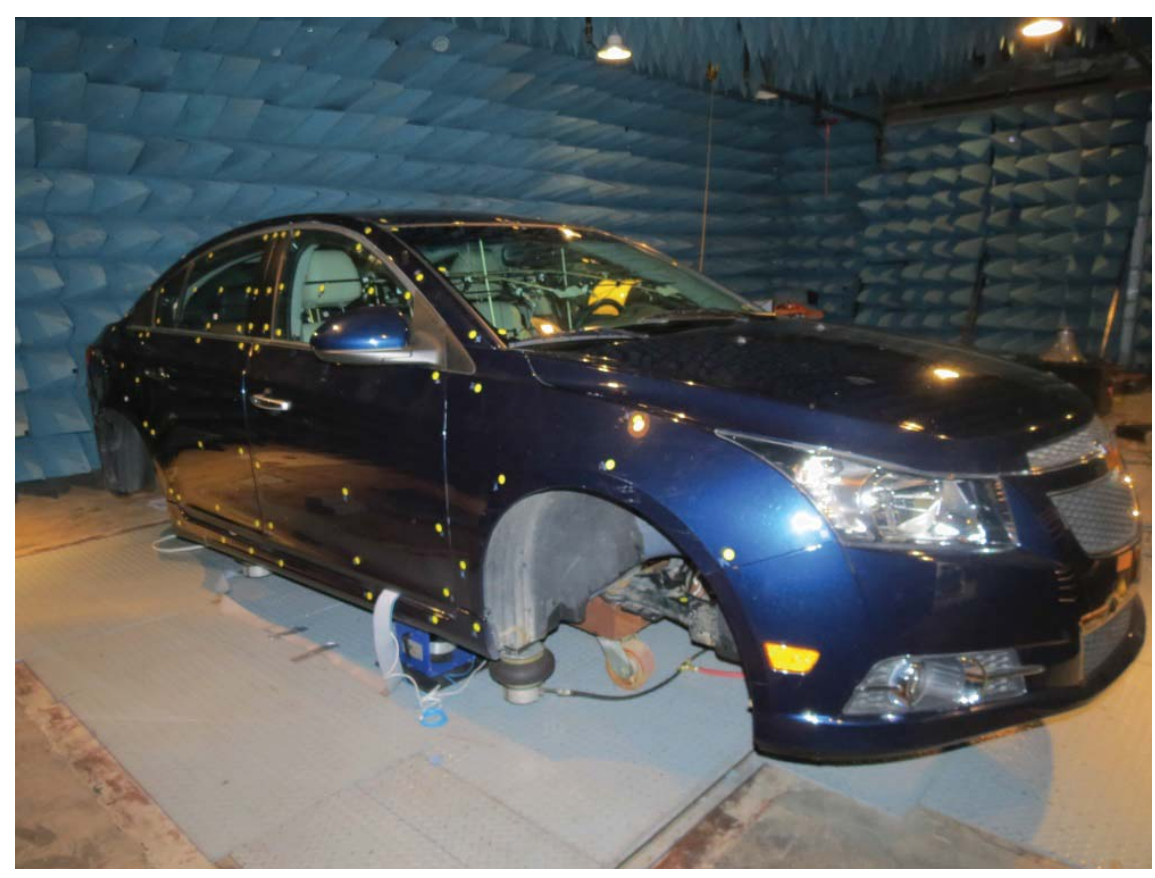

Figure 24: Passenger side view of the points measured on the vehicle 


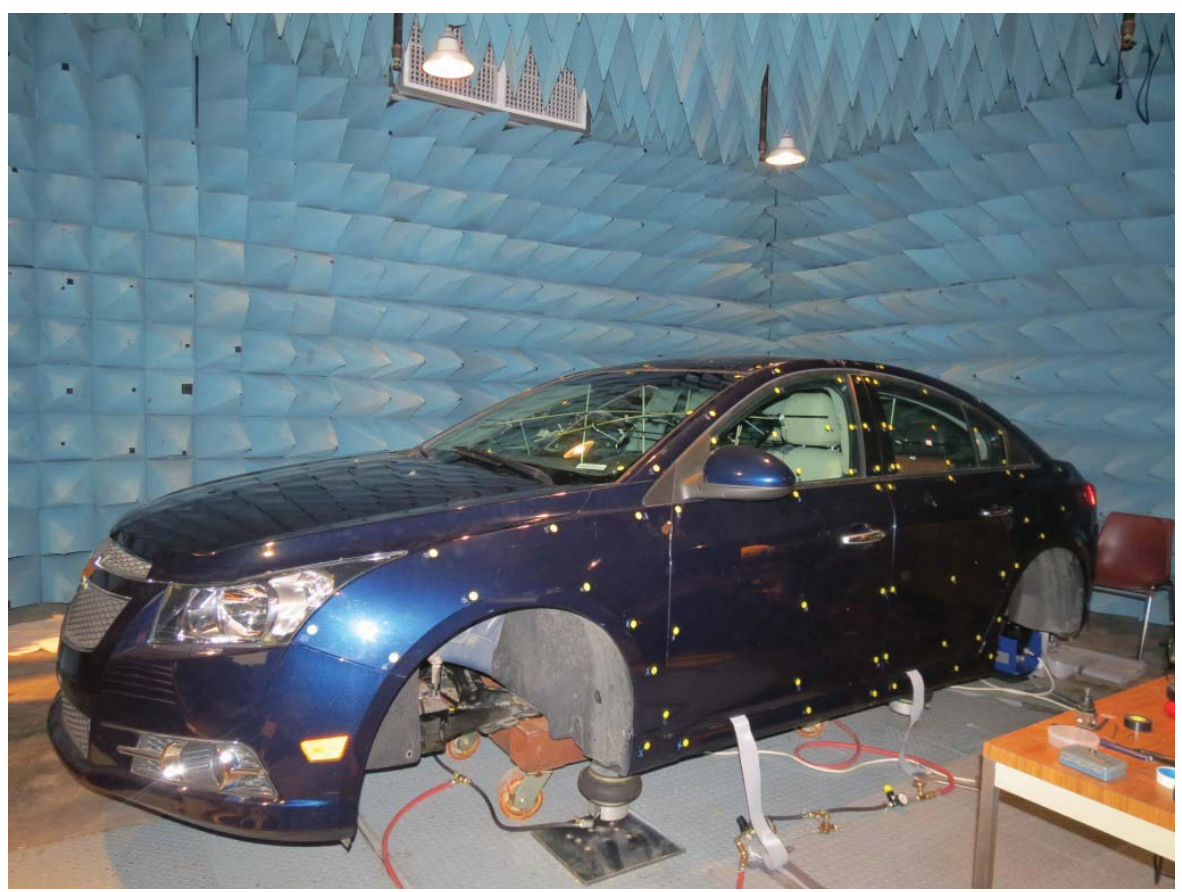

Figure 25: Driver side view of the points measured on the Vehicle

Figure 26 shows the overlay of drive point FRF for the rear shaker from all the runs. It can be seen that the FRFs do not lie exactly over each other, due to small changes in the boundary conditions over time. The phase of these FRFs is between 0 and 180 degrees.

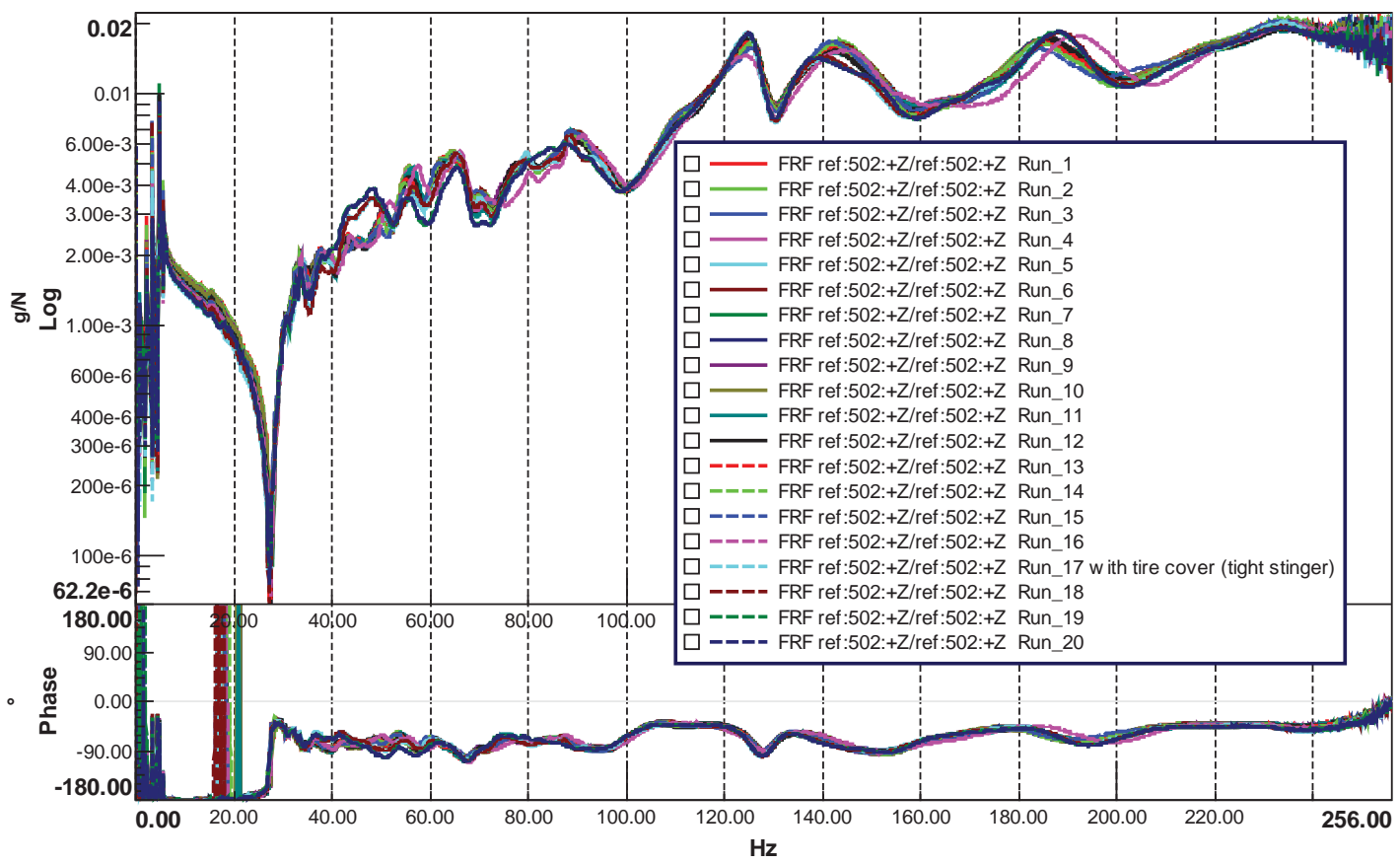

Figure 26: Overlay of Drove Point FRF for the Rear Shaker 
Figure 27 shows the drive point FRF for the front shaker. Comparing this FRF with the one for the rear shaker, it can be seen that the modal density is very high for the front shaker. Due to this, no resonances or anti-resonances can be seen clearly. The phase of the FRF for the front shaker is just below 0 degrees.

Figure 28 shows the acoustic response at the Driver's Outer Ear (DOE) due to the rear shaker. The curves are overlaid from all the runs during the structural test. The acoustic response is generally repetitive for all the runs. In the $40 \mathrm{~Hz}$ region, a peak can be seen indicating an acoustic mode due to a structural input.

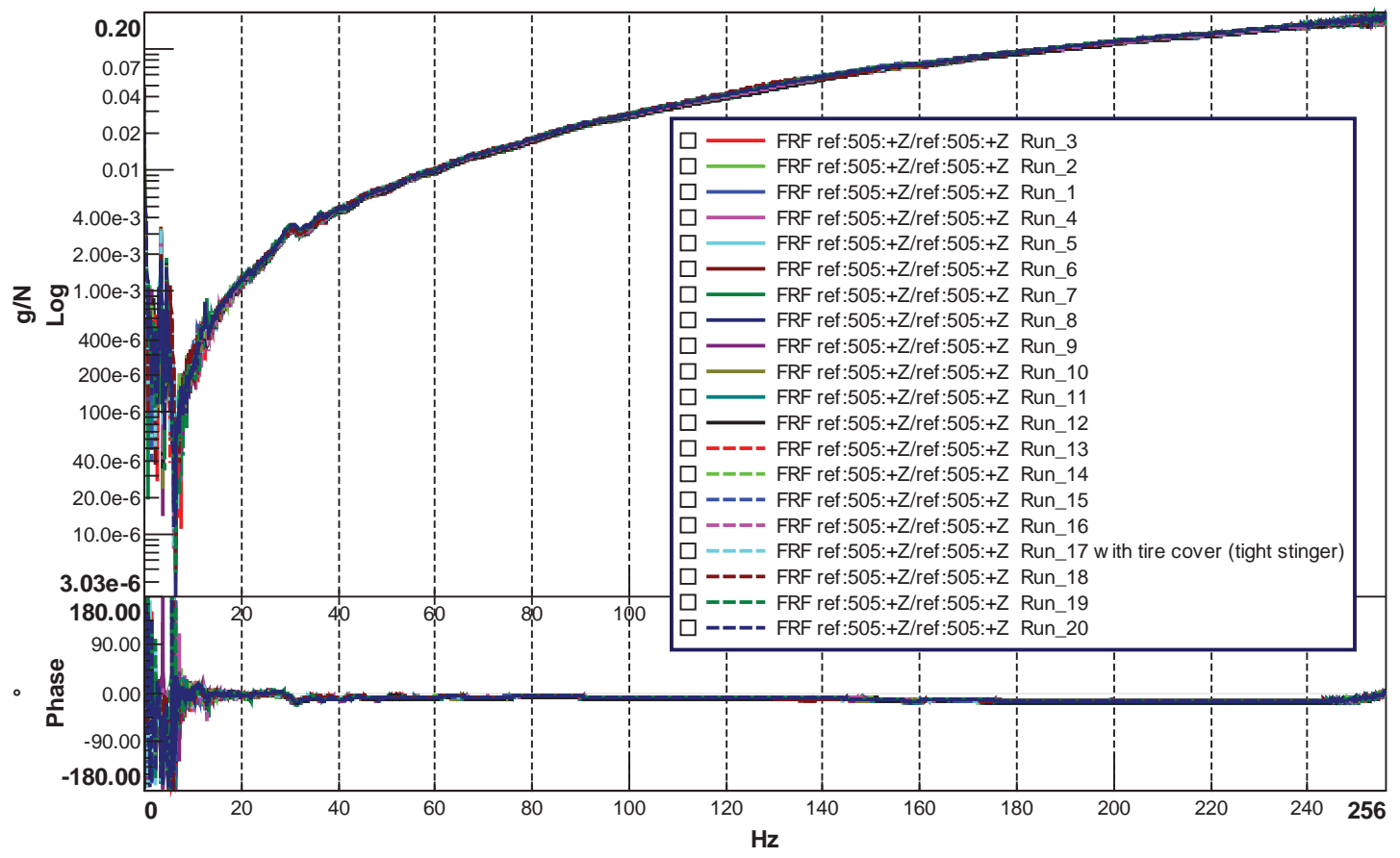

Figure 27: Overlay of Drive Point FRF for the Front Shaker 


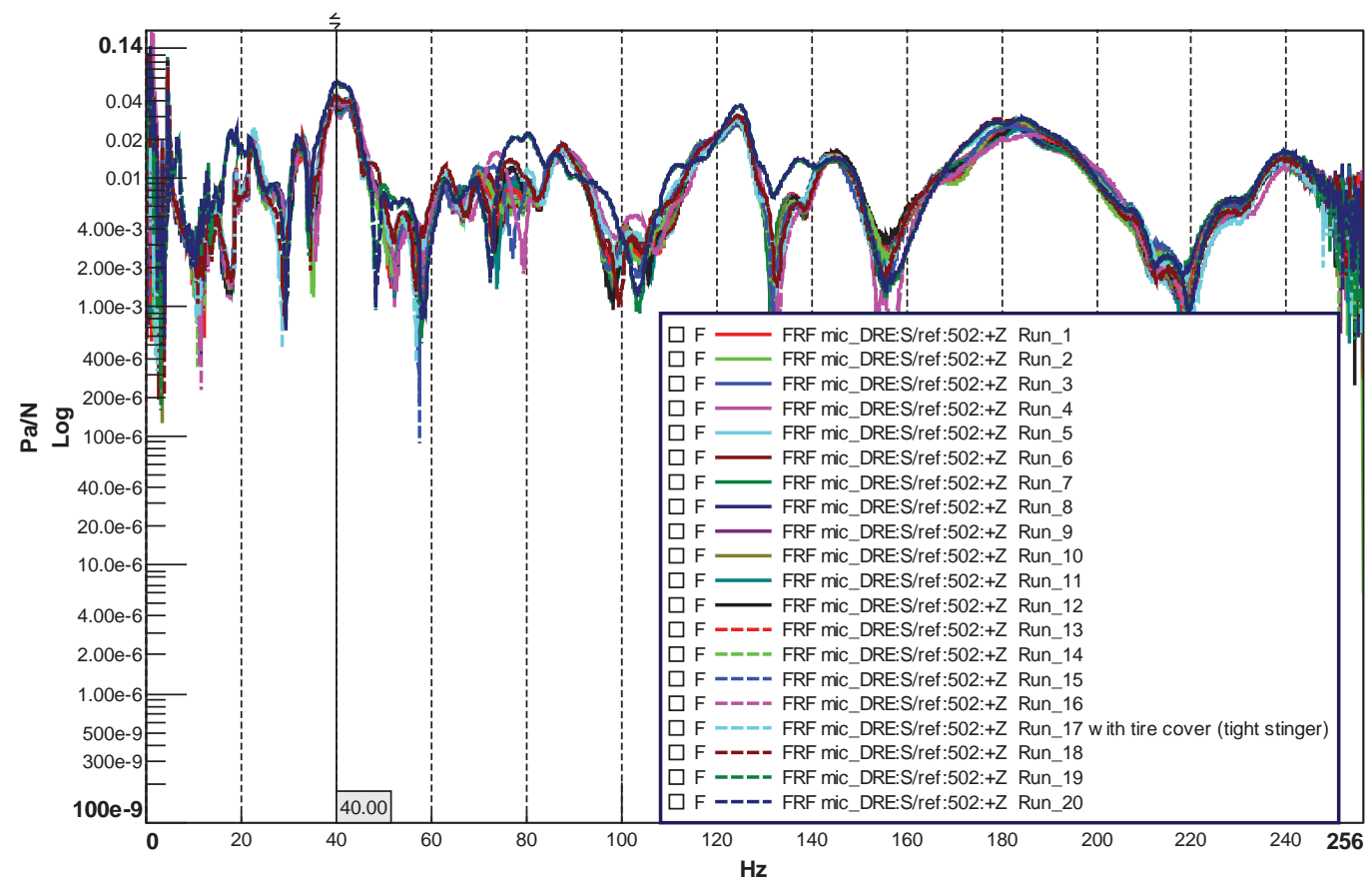

Figure 28: FRF of DRE (Driver's Right Ear) due to input from the Rear Shaker

Figure 28 shows the acoustic response at the Driver's Right Ear due to the front shaker. This plot shows a peak at $40 \mathrm{~Hz}$ as well. Also, it can be seen that there is a sharp rise in acoustic response at DOE from $30 \mathrm{~Hz}$. As this trend is not linear, the booming effect would be more perceivable to the driver.

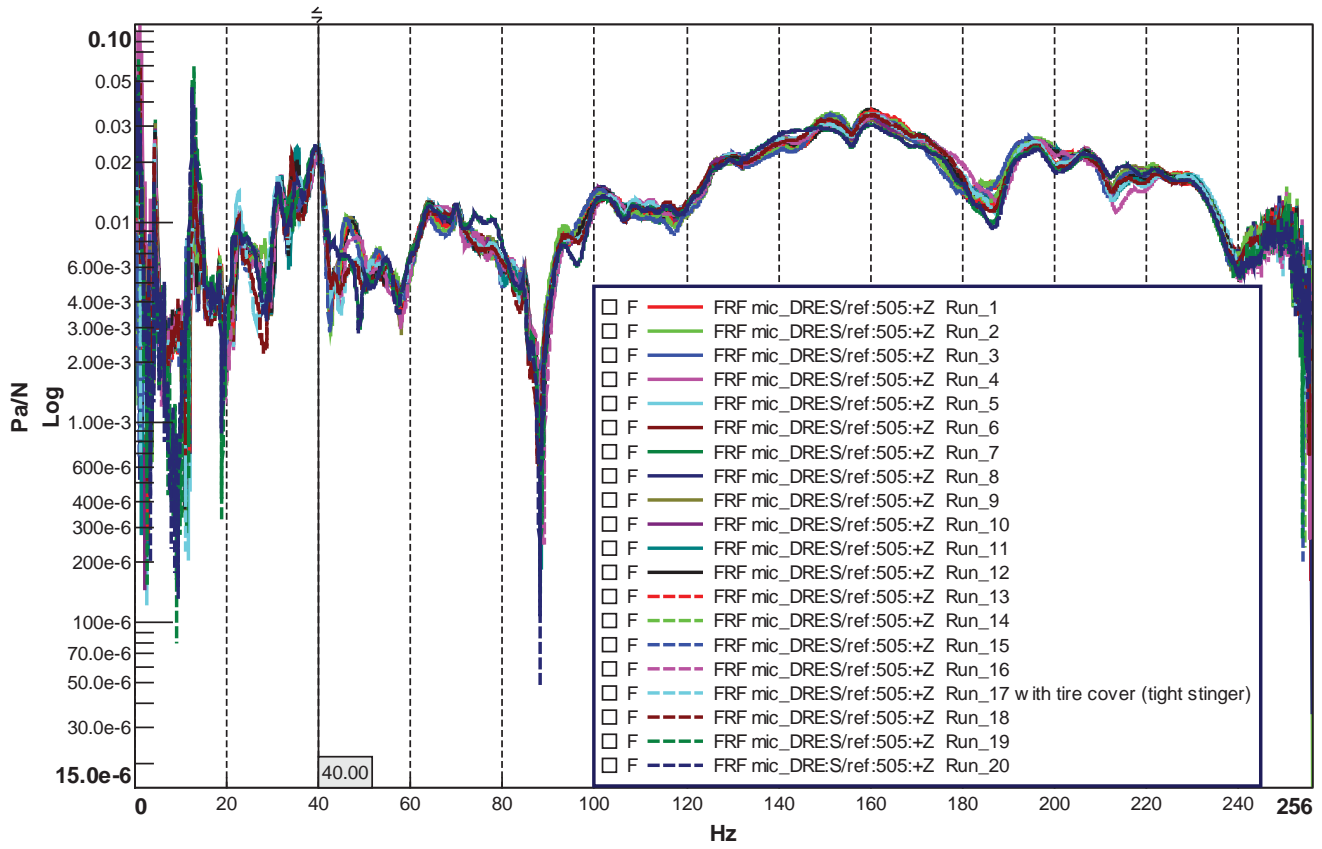

Figure 29: FRF of DRE (Driver's Right Ear) due to input from the Front Shaker 
Once, all the data was acquired, it was curve-fitted to acquire mode shapes. The curve fitting algorithm used for this was PolyMAX which is available in LMS Test.Lab 12. PolyMAX is an algorithm that curve-fits in the z-domain. Due to this, the modal parameter estimation yields excellent results. Figure 30 shows the translational mode at $4.6 \mathrm{~Hz}$ in the $\mathrm{Z}$ direction. The whole vehicle moves vertically upwards and downwards. A slight rotation about the lateral axis ( $\mathrm{Y}$-axis) can also be seen. The original/undeformed shape of the car can be seen as the grey lines. The doors, interior, side trims and the top, hood are colored separately for better visualization.

Figure 31 shows the seats mode at $18.8 \mathrm{~Hz}$. The undeformed shape of the vehicle is shown in grey lines. Some of the components that do not deform are not shown for better visualization of seats. The seats deformation shape is in the lateral direction (Y-axis). The seats sway side to side from their original position. The seat deformation was acquired with three points per seat.

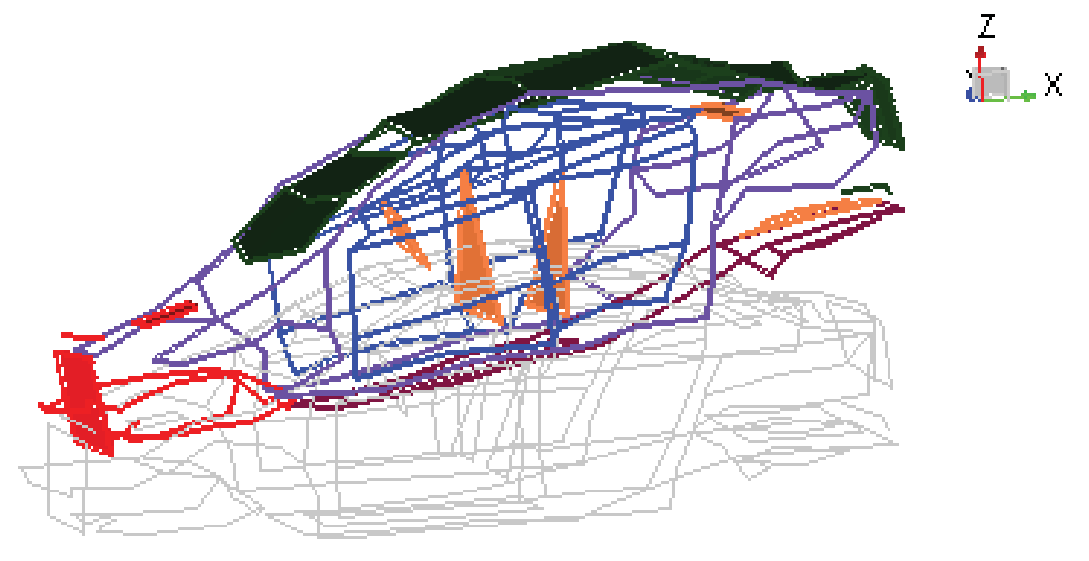

Mode $3: 4.5757 \mathrm{~Hz}, 1.02 \%$ Trans_Z

Figure 30: Translational Rigid Body Mode at $4.6 \mathrm{~Hz}$ 


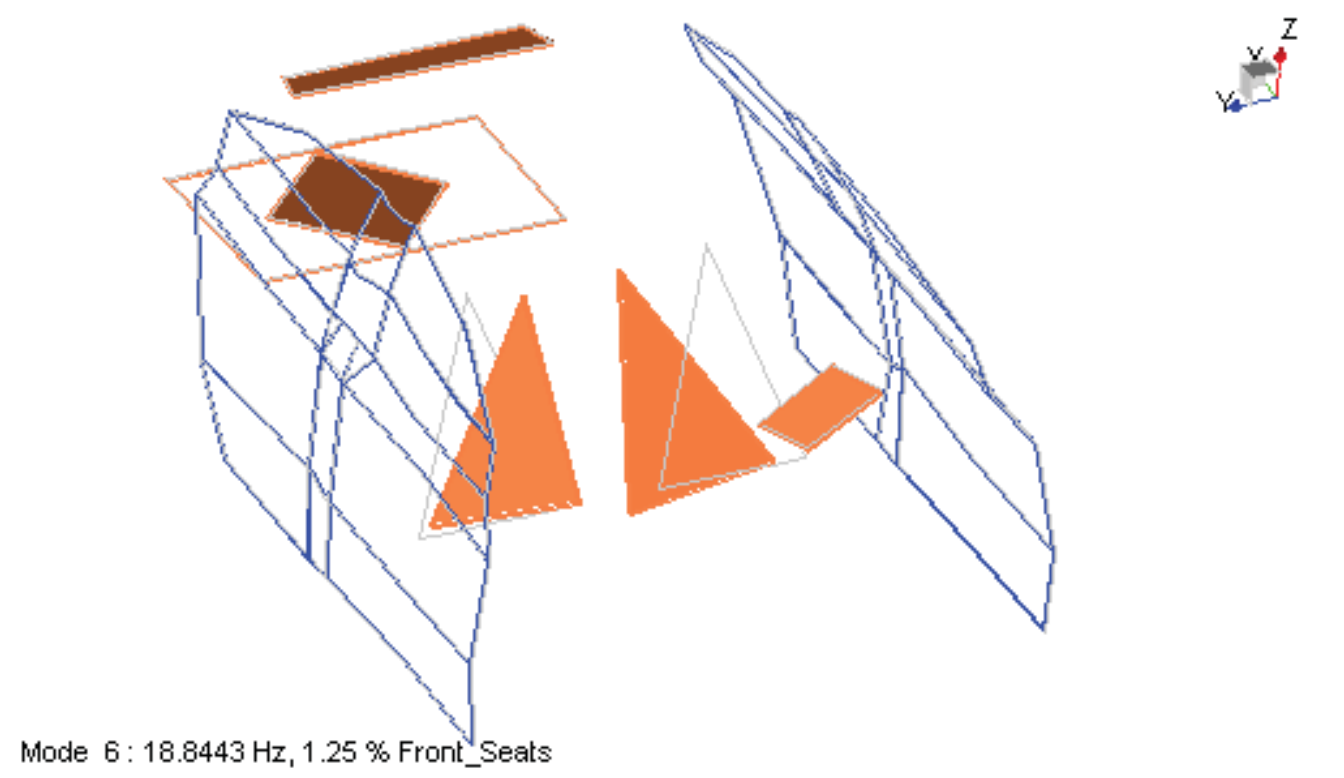

Figure 31: Front Seats Mode at $18.8 \mathrm{~Hz}$

Figure 32 shows the spare tire mode at $22.3 \mathrm{~Hz}$. The data was acquired on the steel rim where the spare tire is installed. The undeformed shape can be seen in black lines. The deformation is in the $\mathrm{Z}$ direction.

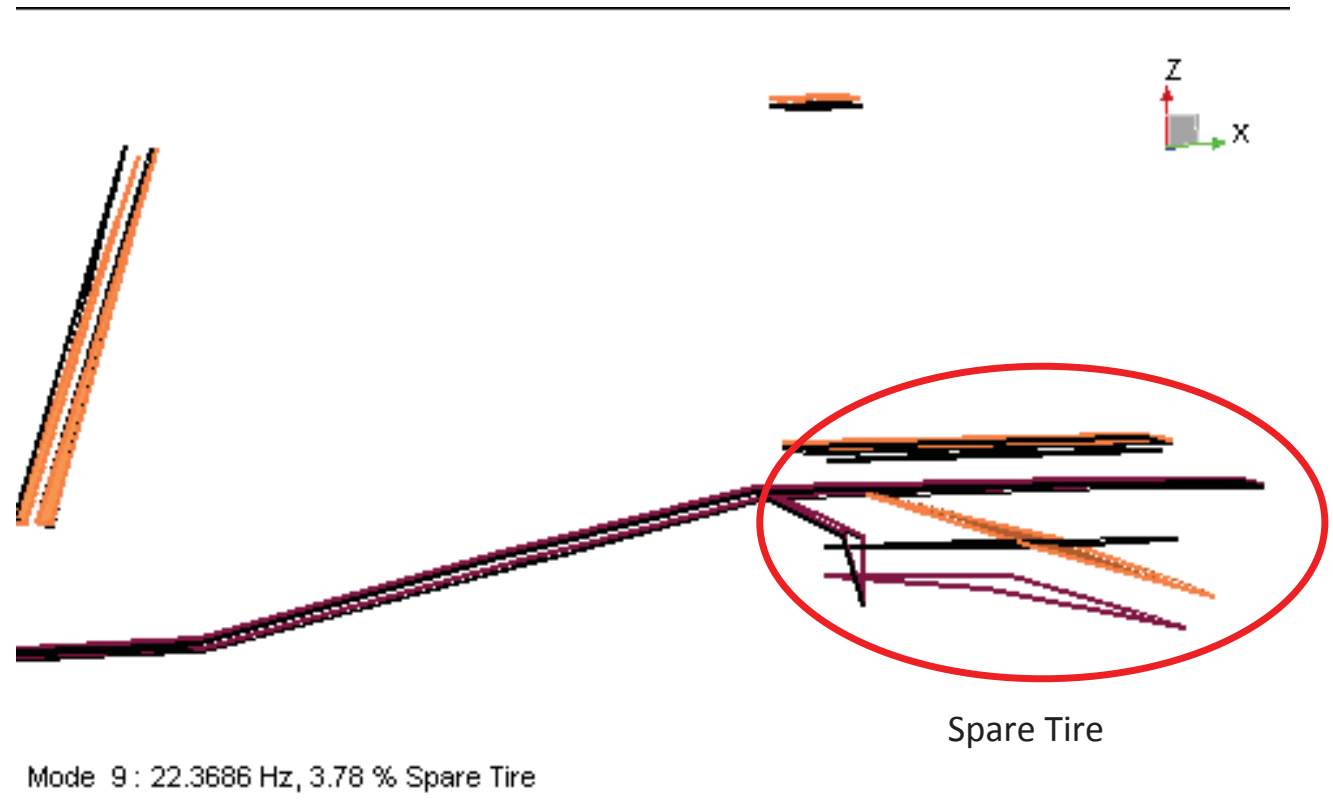

Figure 32: Spare Tire Mode at $22.3 \mathrm{~Hz}$

Figure 33 shows the mode in the frequency region of interest- $40 \mathrm{~Hz}$. This mode is at 42.8 $\mathrm{Hz}$ where the front header and trunk show high motion. 
The doors also move side to side in phase with each other (not seen in figure below). The front header and trunk pump air into the cavity alternately.

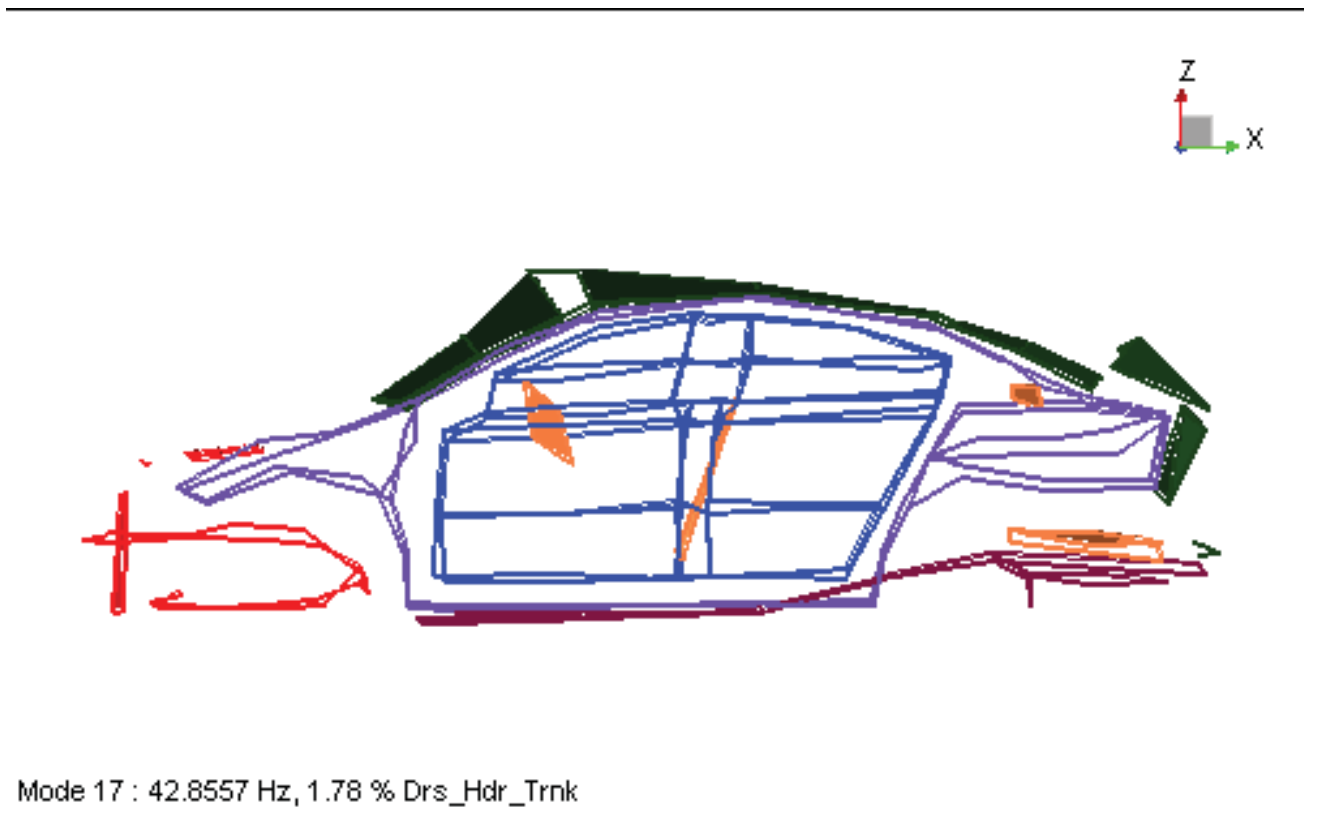

Figure 33: Front Header and Trunk Mode at $42.8 \mathrm{~Hz}$

The phenomenon of acoustic boom occurs when the air moves in the vehicle cabin. For example, consider a closed hollow tank with water filled inside it. If the tank is shaken from side to side, the water also moves along with it. There will be pressure changes inside at any point. Similarly, in the vehicle cabin pressure changes will occur when the air moves due to a particular mode, especially when the air is compressed inside. From the above figure, it can be seen that the front header as well as the trunk push the air inside the vehicle cabin. This movement of air causes the change in pressure in the air cavity. The above mode shape is the structural interaction of the vibro-acoustic coupling. The acoustic interaction will be discussed in the next section.

Figure 34 shows the structural mode at $47.4 \mathrm{~Hz}$. In this mode, high participation from the rear header center and trunk is seen. The doors also participate in this mode. Moreover, the rear header and the trunk also move in phase to compress the air cavity in the vehicle cabin. This is another boom mode of interest in the $40 \mathrm{~Hz}$ range. Other components which do not show much participation have not been shown in the figure below for better visualization. Some participation is also seen from the front header; however, it is comparatively lower than the rear header. 


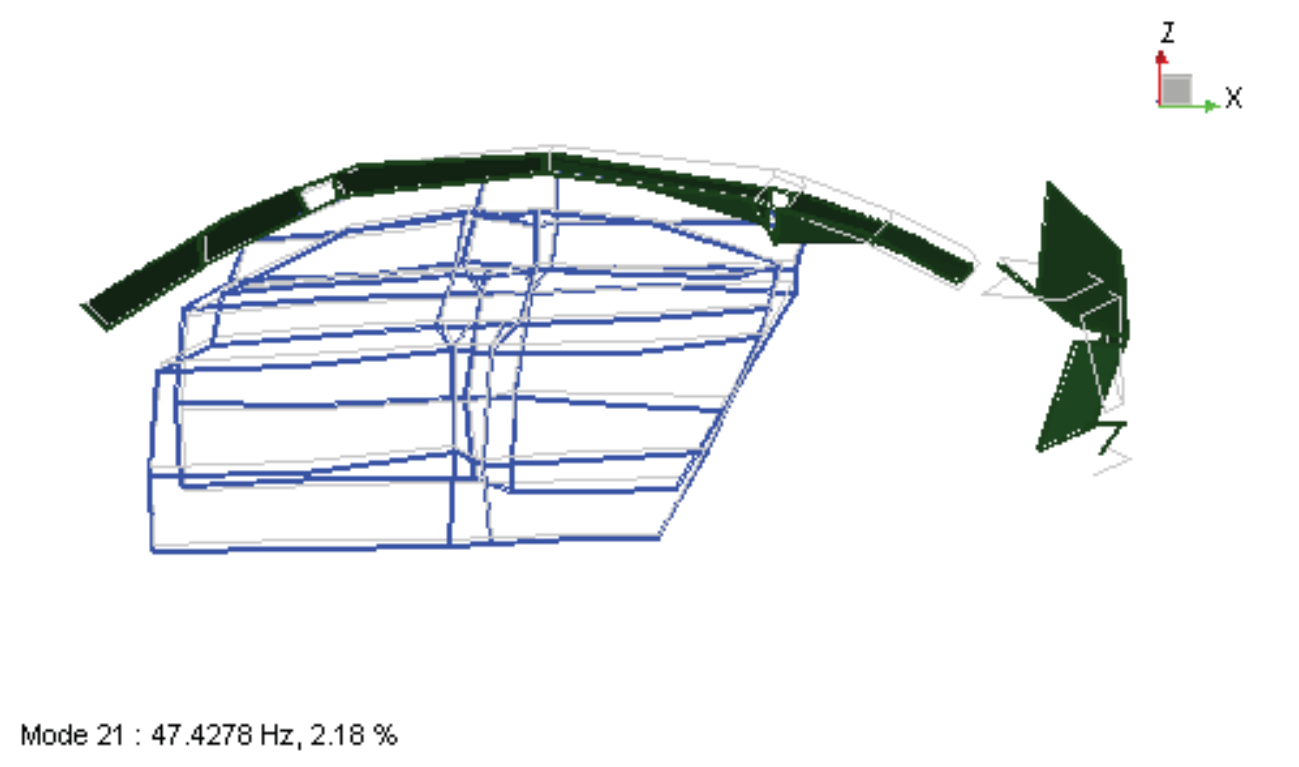

Figure 34: Structural Mode at 47.4 Hz-Rear Header and Trunk Mode

Figure 35 shows the MAC of the structural mode set with it (Auto-MAC) till $65 \mathrm{~Hz}$. The modes are generally independent from each other except in the $30 \mathrm{~Hz}$ and $39 \mathrm{~Hz}$ range where there contribution from neighboring modes. The Auto-MAC tells us that the spatial resolution throughout the structure was sufficient for modes up to $65 \mathrm{~Hz}$.

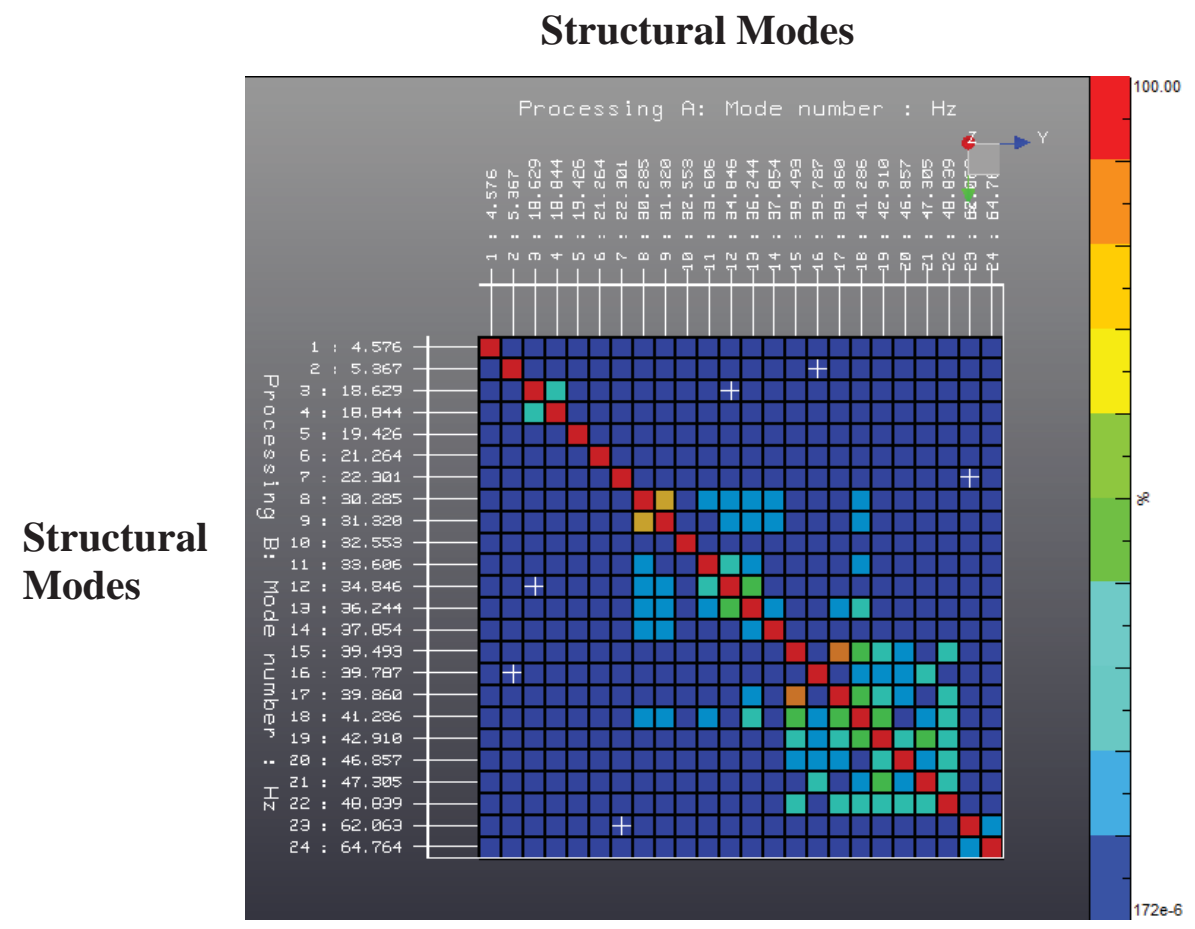

Figure 35: Auto-MAC of Structural Modes till $65 \mathrm{~Hz}$ 


\subsection{Summary of Structural Modal Test}

The full structural modal test was successfully performed on the Chevrolet Cruze LTZ. Data from 286 points was acquired from the test so as to get a good spatial resolution while viewing the mode shapes. The acoustic response at DRE was also measured during the test. The response at DRE showed a peak in the $40 \mathrm{~Hz}$ range. The mode at $42 \mathrm{~Hz}$ shows high participation from the front header and the trunk. Some participation from the doors was also seen. Also, at $47.4 \mathrm{~Hz}$, the rear header and the trunk show high motion. This test confirms that there is structural participation in the $40 \mathrm{~Hz}$ boom range. 


\section{Full Acoustic Modal Test - Structural Input}

\subsection{Purpose}

The structural modal tests informed about the behavior of the vehicle structure in the frequency range of interest. The acoustic modal tests would give information on the behavior of the cavity due to the vehicle structure. Global information of the vehicle cavity would be obtained with the help of acoustic mode shapes.

\subsection{Description}

An acoustic grid was created in the vehicle cabin and the trunk. Together, a total of 52 microphones were used to acquire the data. The acquisition parameters used in this test were the same as used in the structural test. Figure 36 shows the acoustic grid in the front seats.

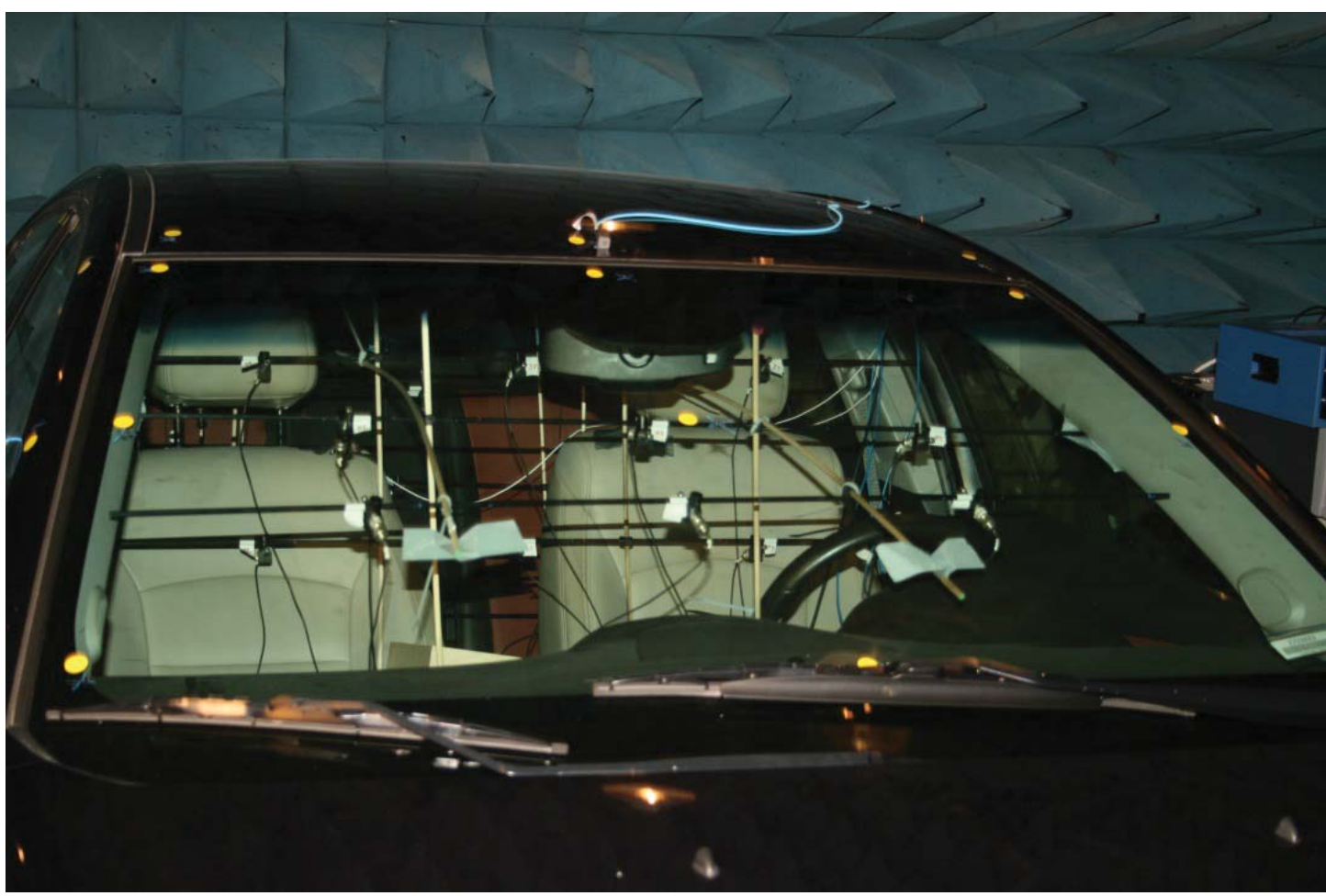

Figure 36: Acoustic Grid in Vehicle Cabin (Front Seat) 
Figure 37 shows the acoustic grid in the rear seats and the trunk of the vehicle.
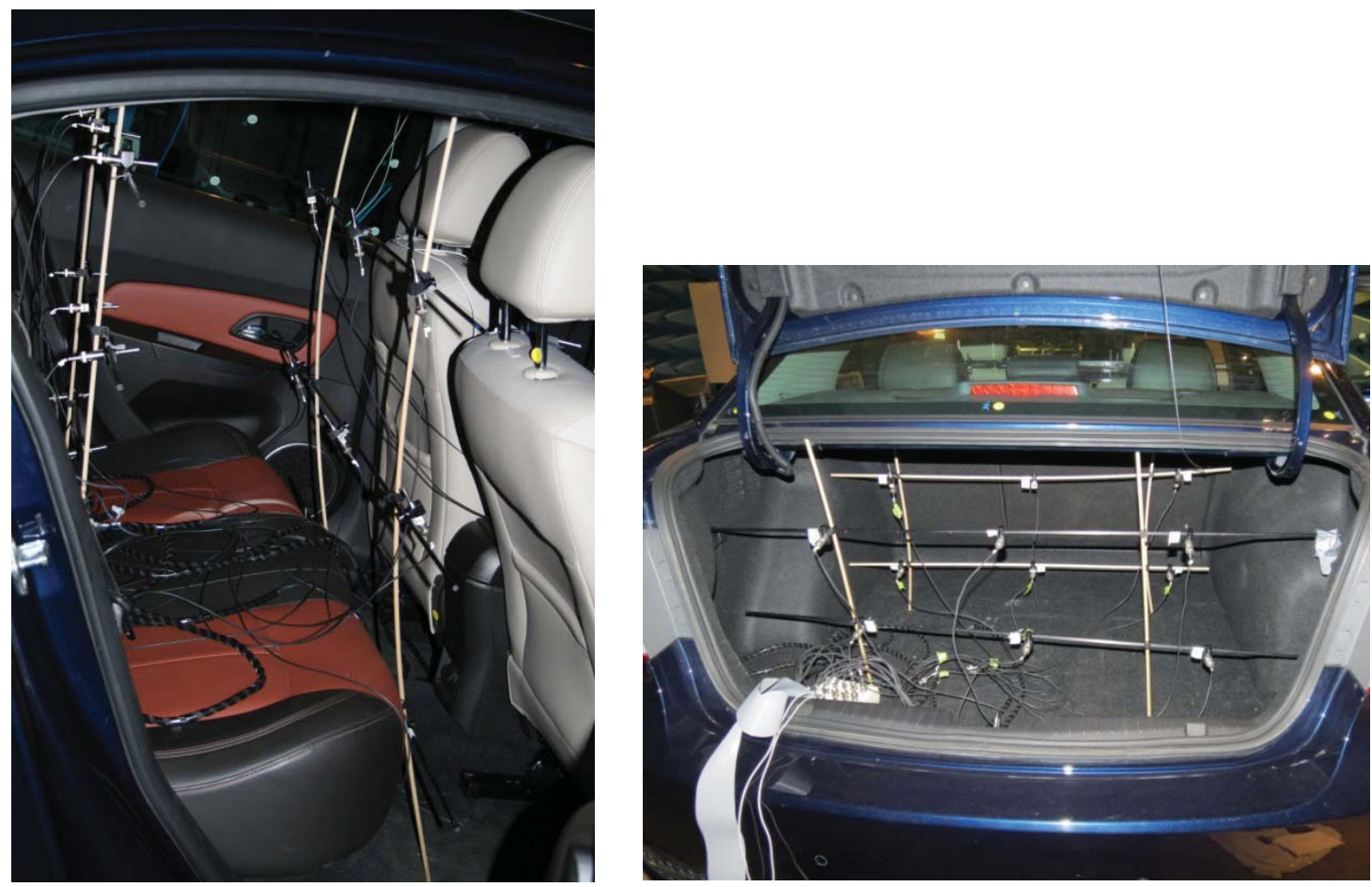

Figure 37: Acoustic Grid in Vehicle (Rear Seats and Trunk)

Figure 38 shows the acoustic response at the Driver's Right Ear (DRE) to the shaker input (both rear and front). It is important to note that due to shortage of channel count, the position of DRE was changed by a small amount. Figure 3 shows the location of DRE used during the structural modal test. In this test, a microphone from the acoustic grid was used as the DRE location. Microphone 38 (Cavity: 38) was used as the location of DRE. From the figure below, it can be seen that the mode at $40 \mathrm{~Hz}$ is captured by the new location as well. The response by the rear shaker is the red curve while the response due to front shaker is the blue curve.

From the full structural modal test, it was observed that the front header, rear header and the trunk show high participation in the boom range $(40 \mathrm{~Hz})$. Hence, it would be of great interest to know the acoustic response at these locations in the full acoustic modal tests as well. As the input to the structure is the same (through shakers), it would be expected that the microphones close to these locations would show a high acoustic response. Figure 39 shows the acoustic response below the front and rear header centers. The coherence function at these locations is also shown. It can be seem from Figure 39 that the boom mode in the $40 \mathrm{~Hz}$ range is captured for both the front and rear headers. The structural input for these curves is the rear shaker. The coherence function is close to unity indicating a good linear response to the input. 


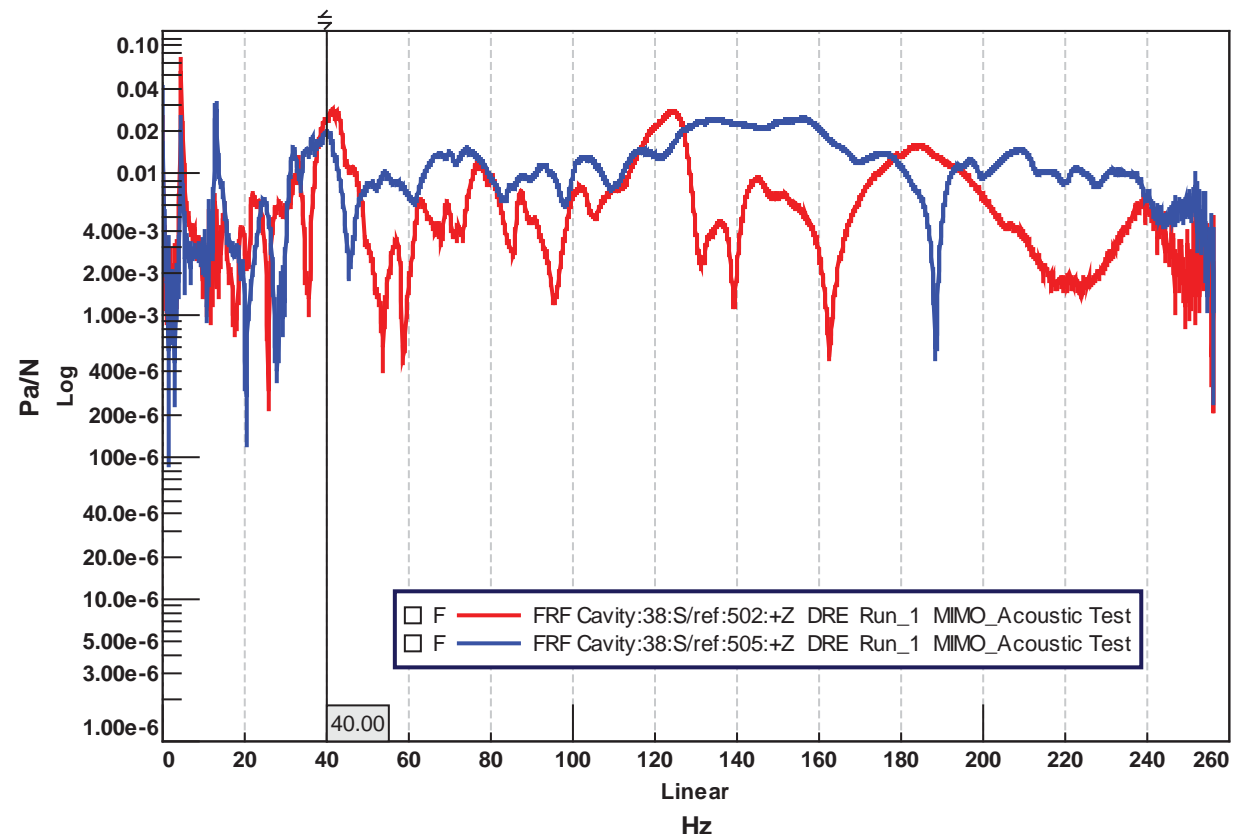

Figure 38: Acoustic Response at DRE due to Rear (Red) and Front (Blue) Shaker

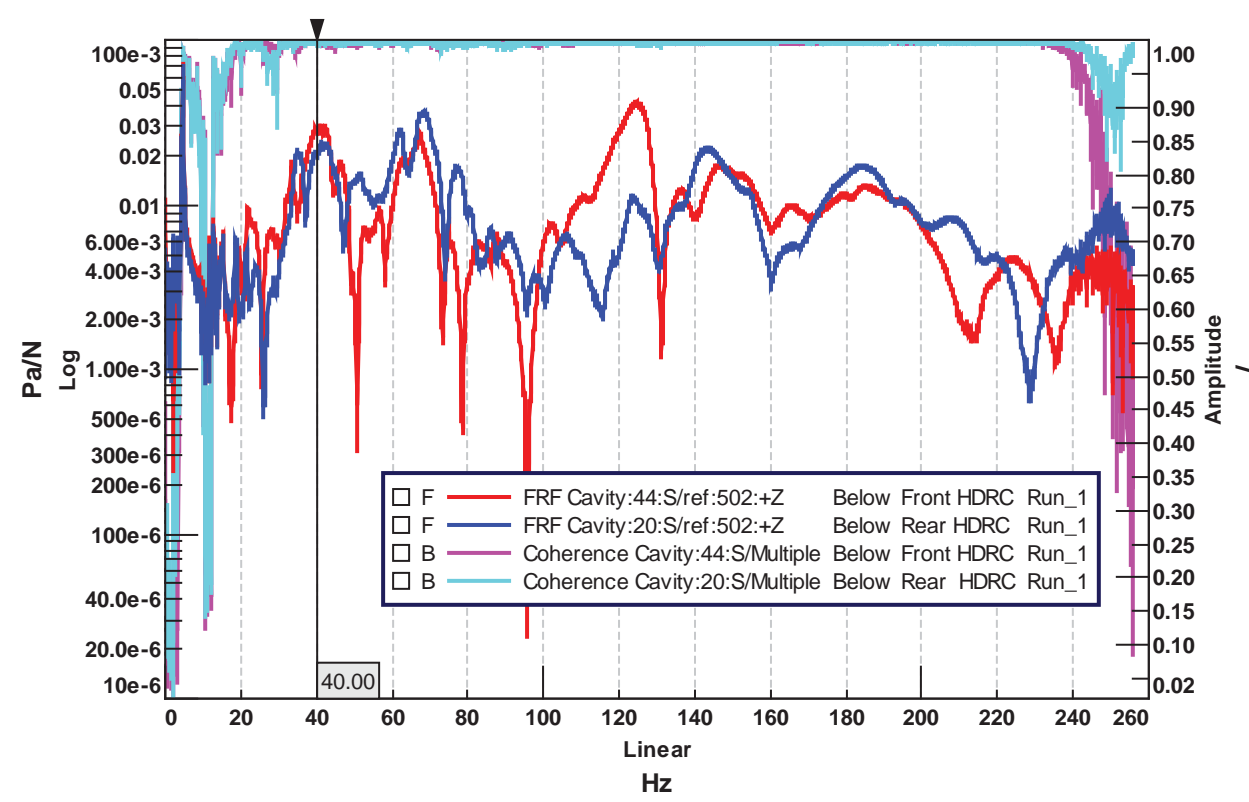

Figure 39: Acoustic FRF and Coherence below Front and Rear Header Centers due to Rear Shaker

Figure 40 shows acoustic response and coherence functions at the front and rear header centers due to structural input from the front shaker. In this case as well, there is high acoustic response in the $40 \mathrm{~Hz}$ boom range. 


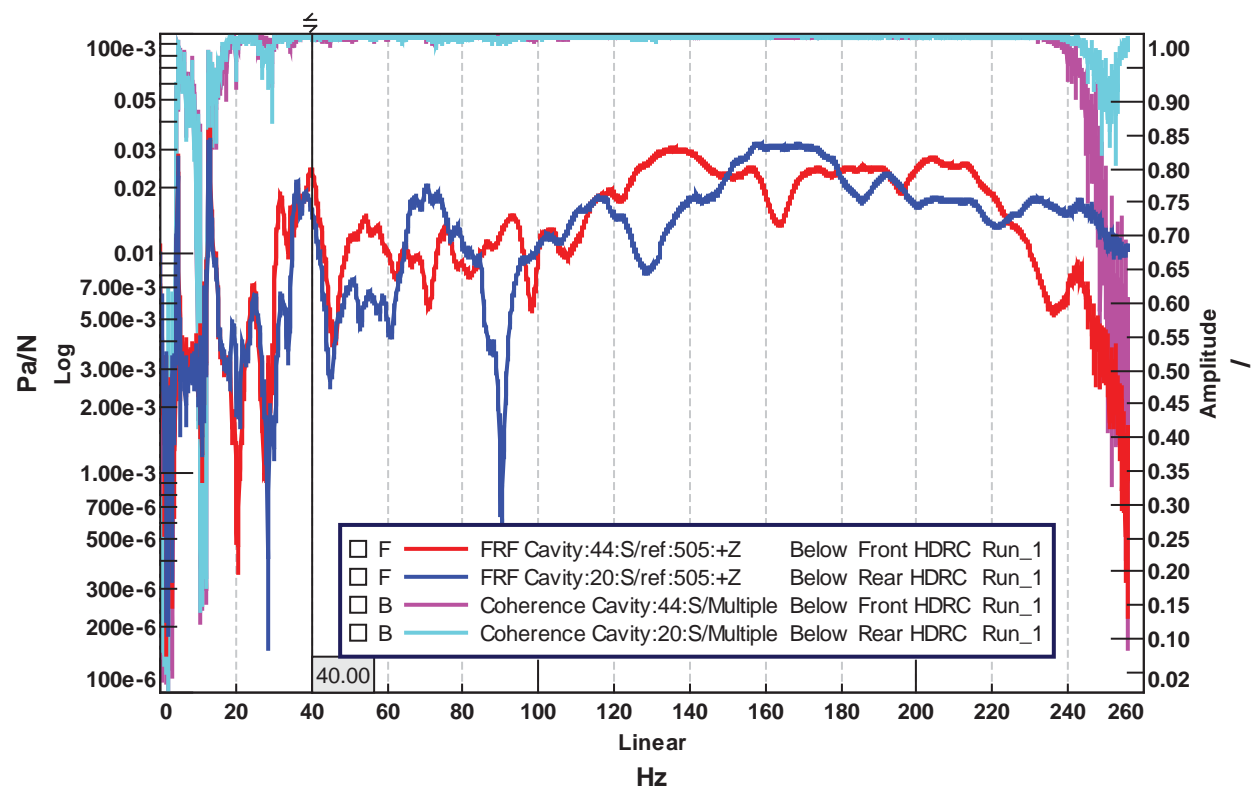

Figure 40: Acoustic FRF and Coherence below Front and Rear Header Centers due to Front Shaker

Figure 41 shows the acoustic response at the trunk. From the structural mode shapes, it was observed that the point at the trunk bottom center showed high motion. Hence, the acoustic point closest to that was chosen to observe the acoustic response.

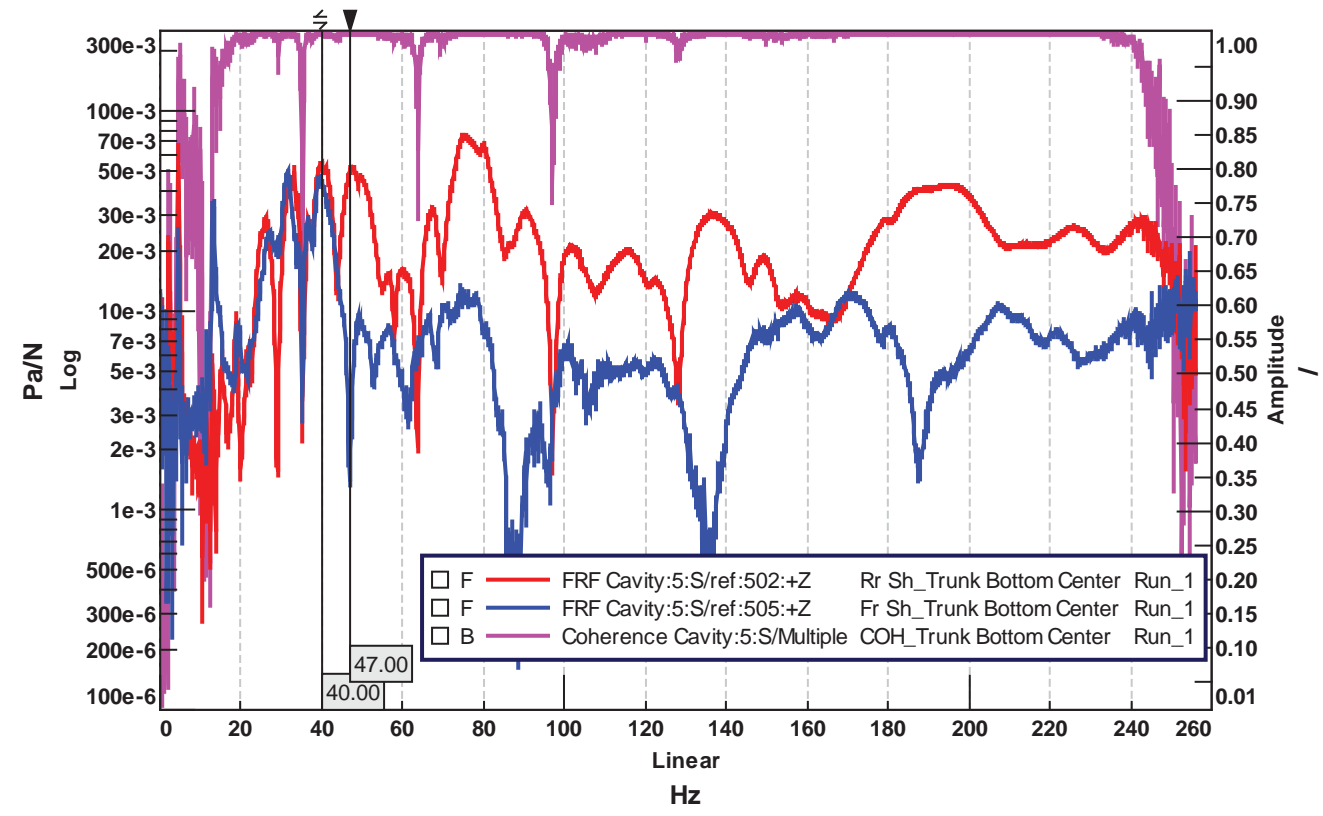

Figure 41: Acoustic FRF and Coherence at Trunk Bottom Center due to Rear (Red) and Front (Blue) Shakers

From Figure 41 it can be seen that the response due to rear shaker has peaks at $40 \mathrm{~Hz}$ and $47 \mathrm{~Hz}$ which are the modes of interest. 
Quite understandably, the front shaker is not able to excite the trunk at $47 \mathrm{~Hz}$ as much as the rear shaker. Hence a lower response is seen by the acoustic response due to the front shaker.

While curve-fitting the FRFs to obtain mode shapes, the structural as well as acoustic responses were curve fitted together to get a better idea of the fluid structure interaction. Hence, the following mode shapes will primarily explain the acoustic pressure distribution in the vehicle cavity while also showing the motion of the vehicle structure.

Figure 42 shows the spare tire mode at $22 \mathrm{~Hz}$. The undeformed shape is shown with the grey lines. As the spare tire region moves upwards, a high pressure develops in the trunk. When it moves downwards, a low pressure is generated. Pressure equilibrium is created in the vehicle; when there is high pressure in the vehicle cabin, there is low pressure created in the trunk. Similarly, when there is high pressure created in the trunk, a low pressure exists in the vehicle cabin. In this mode shape, it seems that the structure is driving the acoustic pressure inside the vehicle.

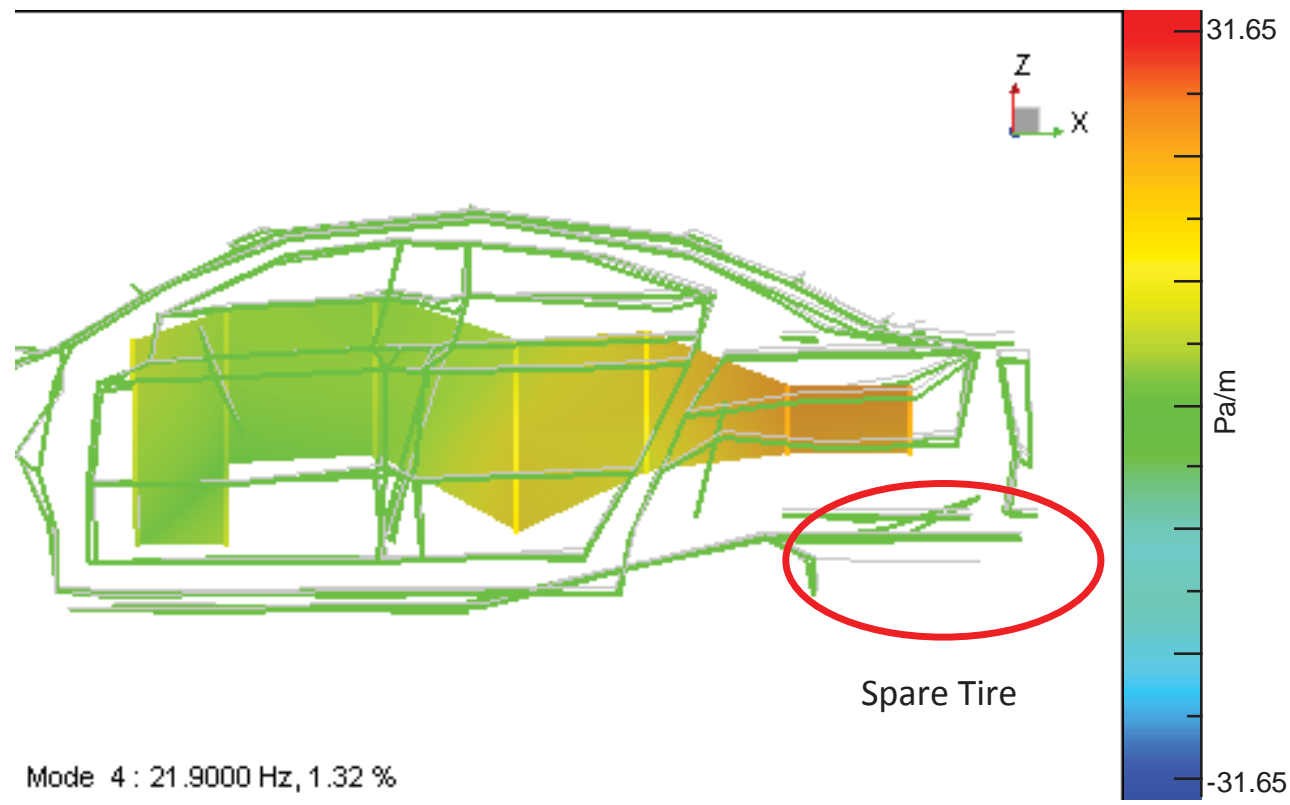

Figure 42: Acoustic Mode at $21.9 \mathrm{~Hz}$

Figure 43 shows the acoustic mode at $40.7 \mathrm{~Hz}$. From the structural mode test it was seen that the front header and trunk had high contribution. We can see from Figure 43 that the front header has moved up and the pressure in the vehicle cabin is negative in magnitude. This mode shape also suggests that the vehicle structure is driving the acoustic pressure inside the vehicle. 


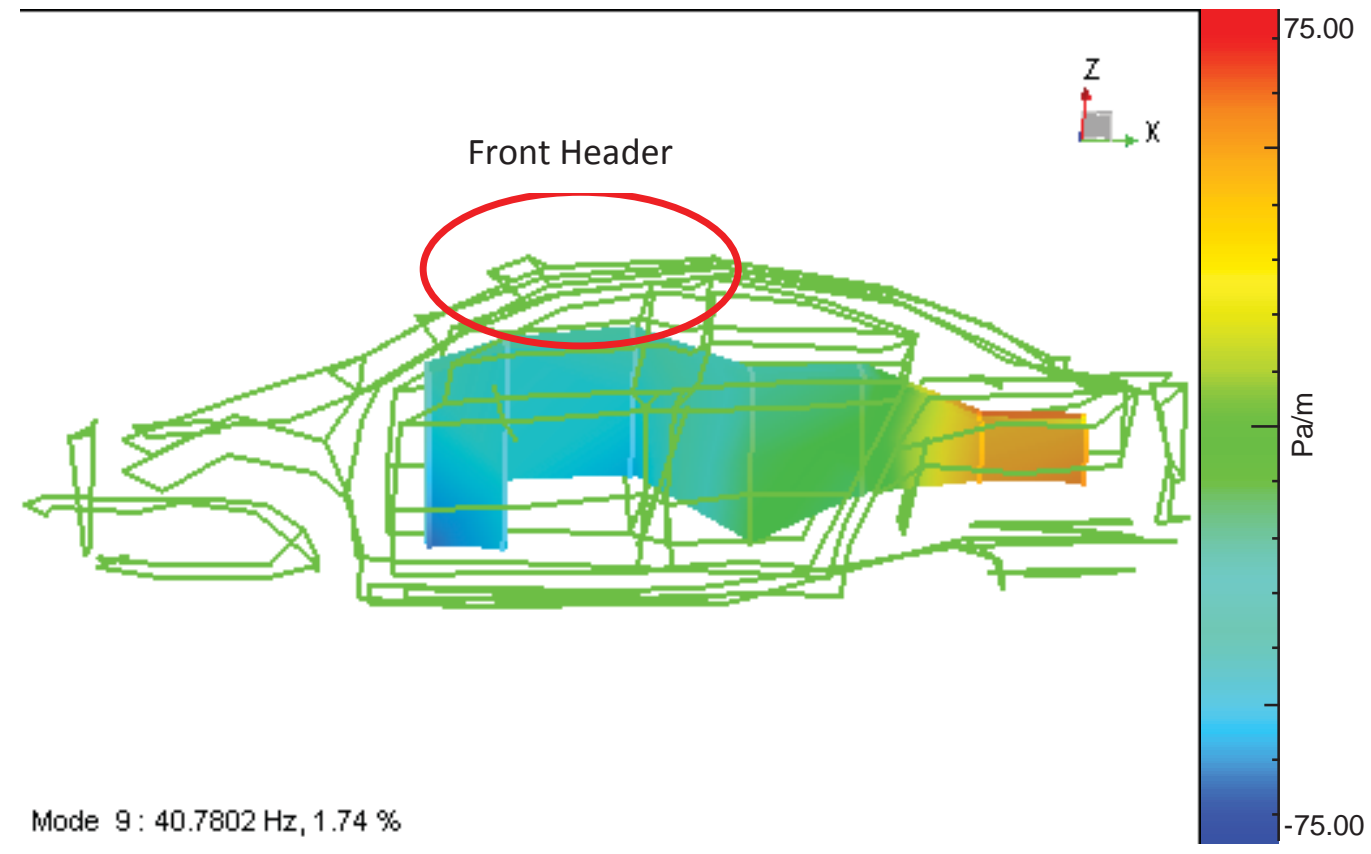

Figure 43: Acoustic mode at $40.7 \mathrm{~Hz}$

Figure 44 shows the acoustic mode at $47 \mathrm{~Hz}$ where high participation was seen from the rear header and the trunk. In this mode, for the acoustic response, the high pressure fluctuation is seen mainly in the trunk than in the vehicle cabin.

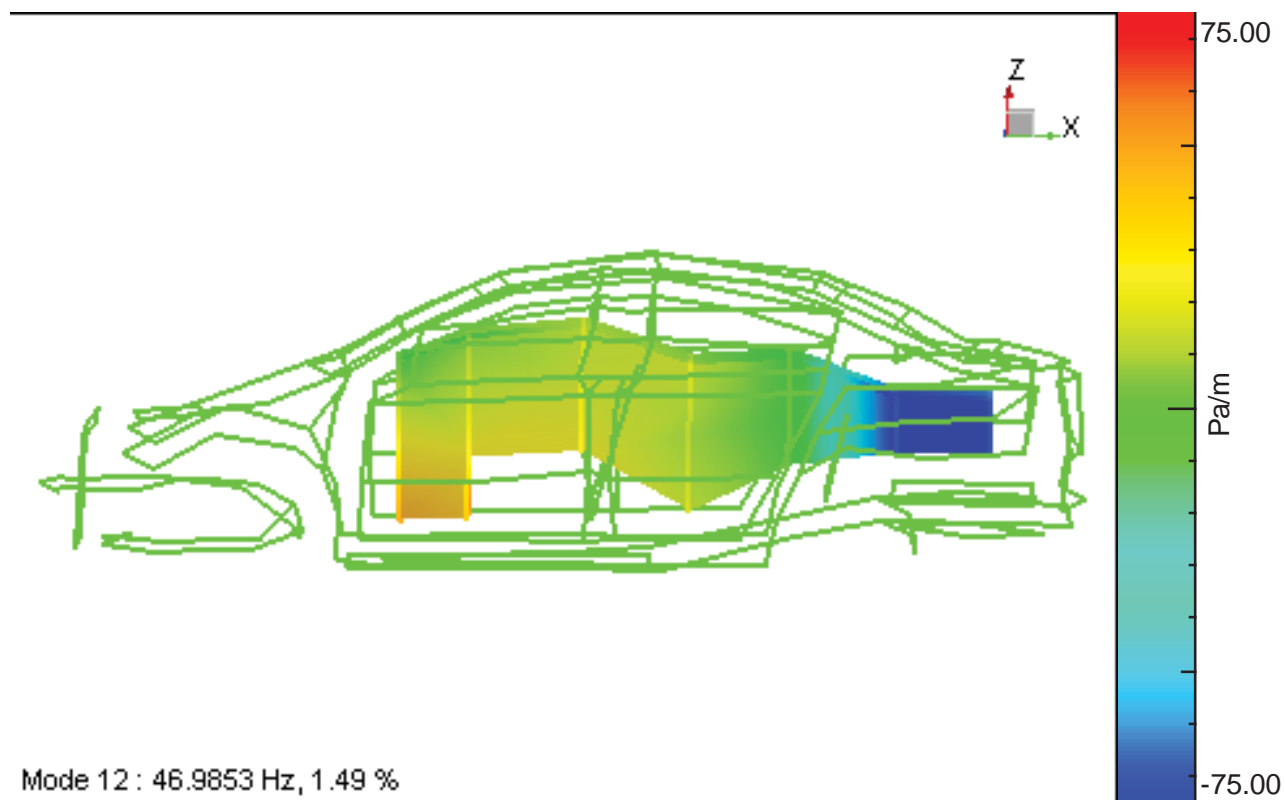

Figure 44: Acoustic Mode at $47 \mathrm{~Hz}$ 


\subsection{Summary of the Acoustic Modal Test with Structural Input}

With the help of this test, the acoustic pressure distribution in the vehicle cabin and the trunk cavity is obtained. The fluid structure interaction due to the structural input is understood. It is observed that for most of the modes, the structure drives the fluid due to which the acoustic fluctuations occur. 


\section{Full Acoustic Modal Test: Acoustic Input}

\subsection{Purpose}

The full structural modal test and full acoustic modal test gave information about the behavior of the vehicle structure and vehicle cavity when the structure was excited through force from the shakers. In order to confirm and glean further information of fluid structure interaction in the vehicle, the vehicle was excited with acoustic inputs. This was done by two speakers; one in the vehicle cabin and the other in the trunk. This was done to mimic the inputs given by the shakers in the earlier tests and also excite the vehicle cavity uniformly. Hence instead of the rear shaker, a rear speaker is used (present in trunk) and instead of a front shaker, a front speaker is used (in between the two front seats). Vibration response at the front header was also measured in this test to measure structural response to the acoustic input from the speakers.

\subsection{Description}

The acoustic grid configuration used in this test was the same as before. Figure 45 shows the speaker in the trunk while Figure 46 shows the speaker used as acoustic excitation in the vehicle cabin. It is placed in between the two front seats. The speaker was mounted on a soft padding which would absorb the vibrations directly from the speaker. This would ensure that the structure was excited only from the acoustic pressure from the speakers. The acoustic absorption of sound due to the speakers (speaker casing is made up of wood) is neglected and assumed to be negligible. As a volume velocity source (VVS) was not used in this test, the reference with which the acoustic and structural responses would be measured would be the voltage input given to the two speakers.

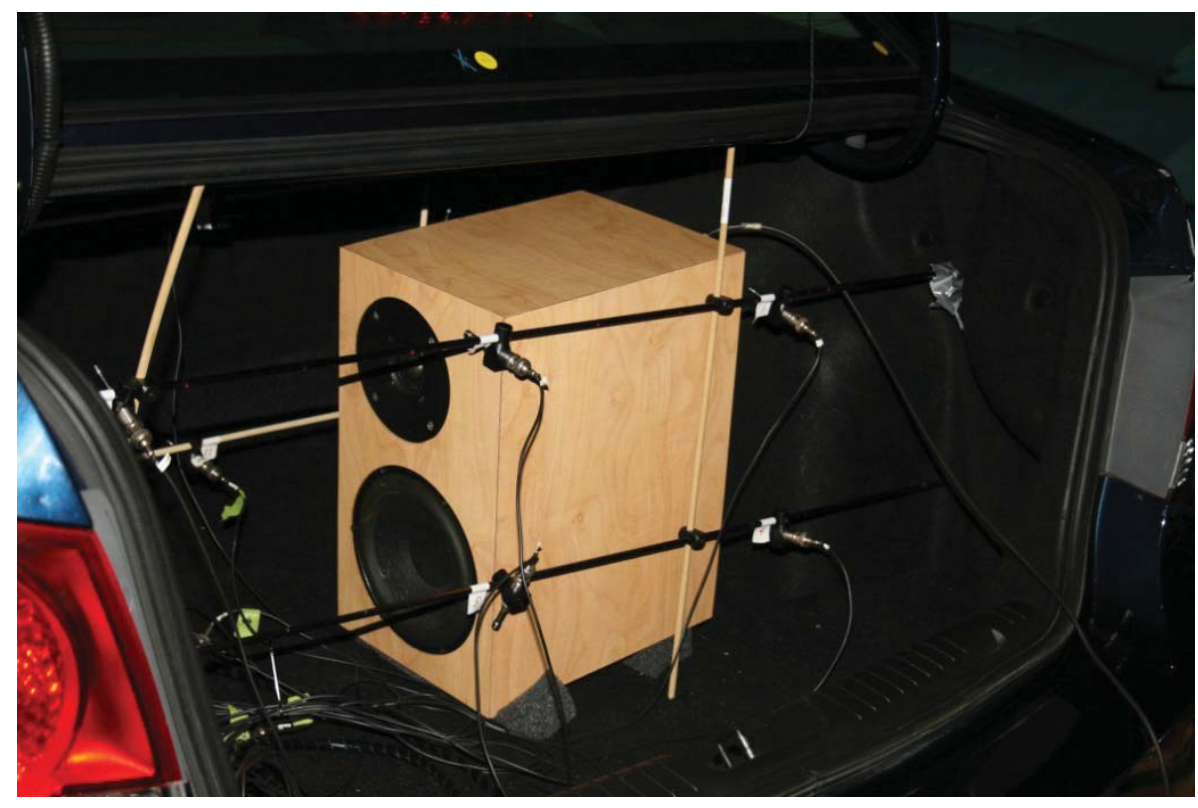

Figure 45: Acoustic Excitation (Speaker) in Car Trunk 


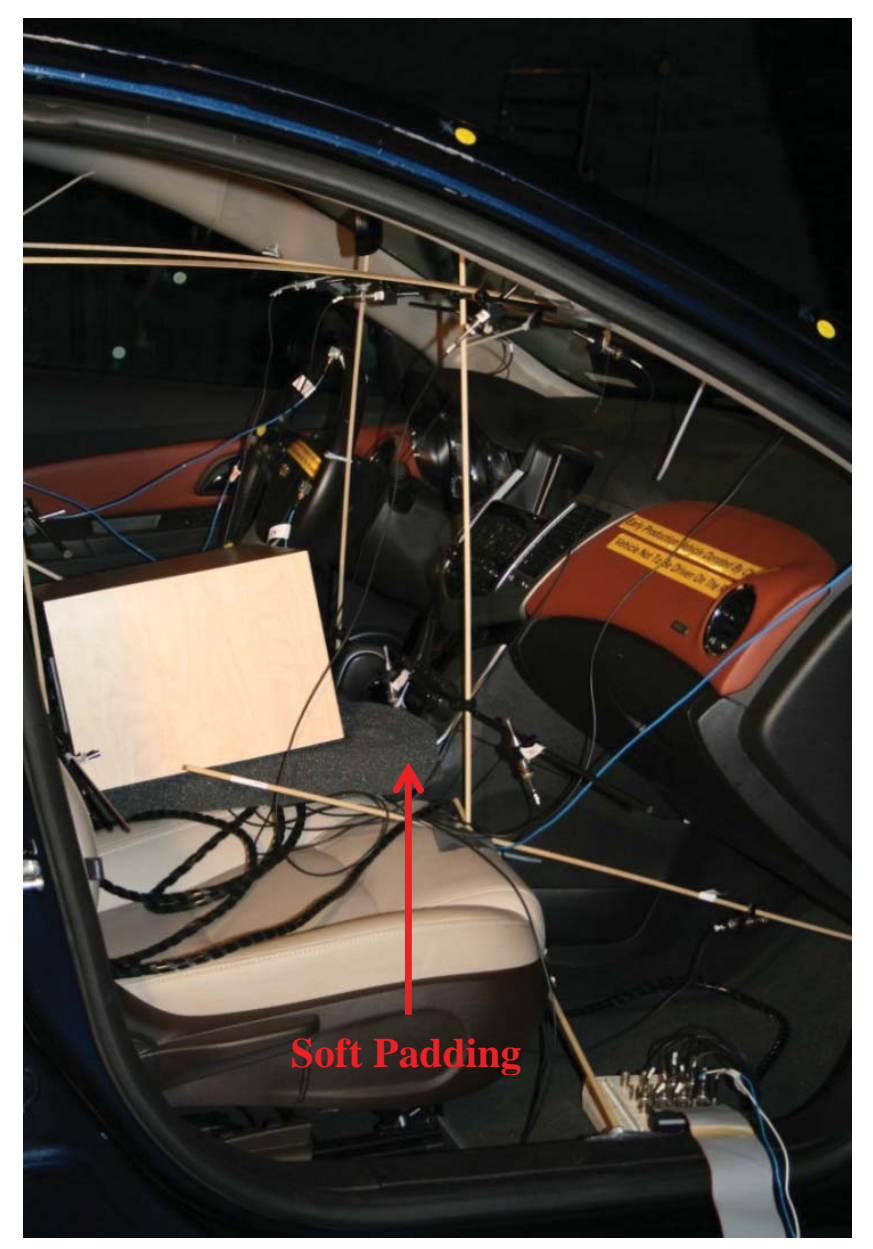

Figure 46: Acoustic Exacitation in Vehicle Cabin (Between Front Seats)

Figure 47 shows the acoustic response at the DRE and structural response at Front Header Center due to the acoustic input from the rear speaker. A clear peak is seen at 40 $\mathrm{Hz}$ both for the acoustic as well as the structural FRF. This confirms the existence of fluid structure interaction or vibro-acoustic coupling in the vehicle.

On the other hand, Figure 48 shows the acoustic and structural FRFs due to input from the front speaker. The structural response shows a clear peak at $40 \mathrm{~Hz}$; however the acoustic peak cannot be seen clearly. This may be due to high modal density in the region. 


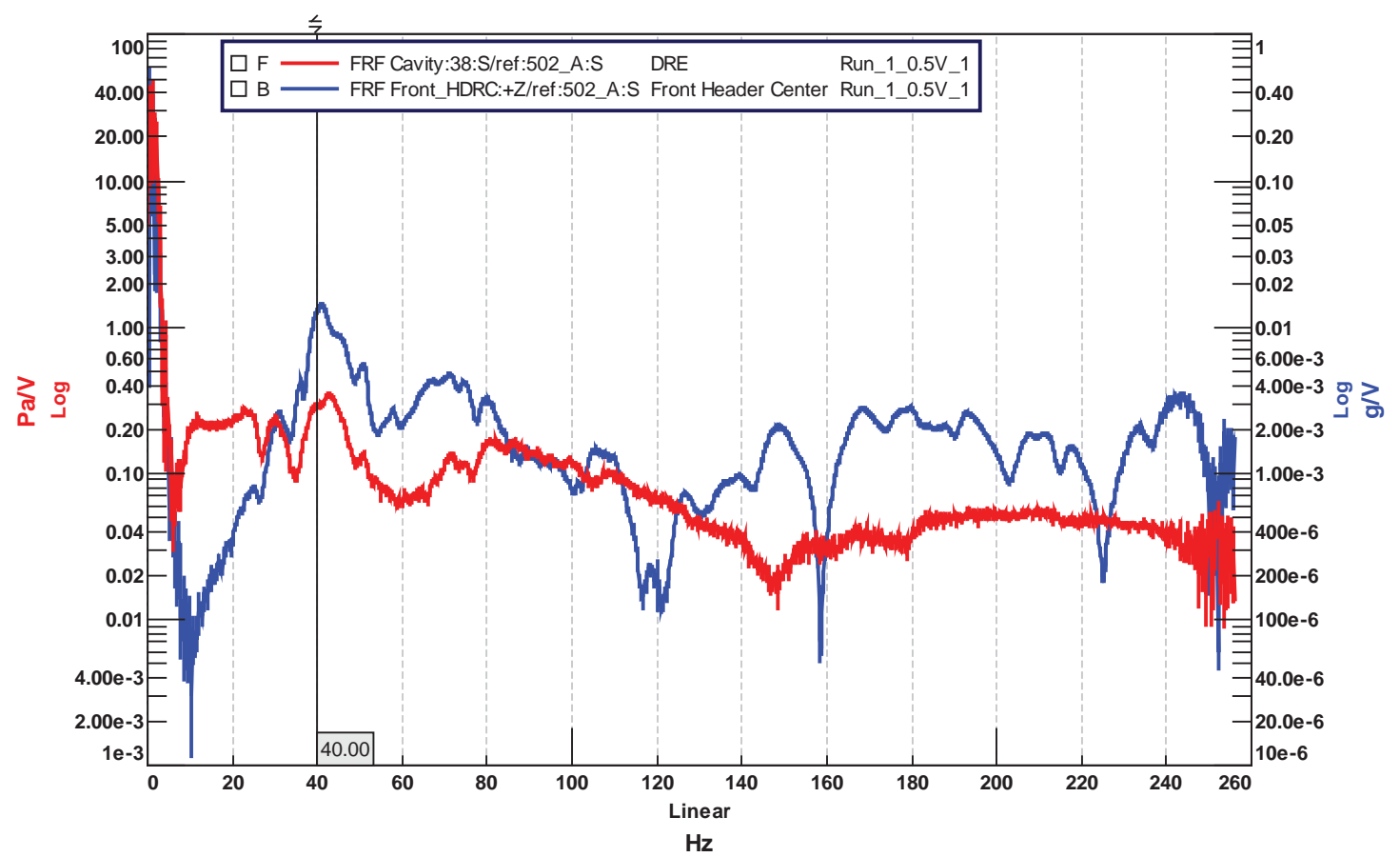

Figure 47: Acoustic FRF at DOE and Vibration FRF at Front Header Center with Acoustic Input at

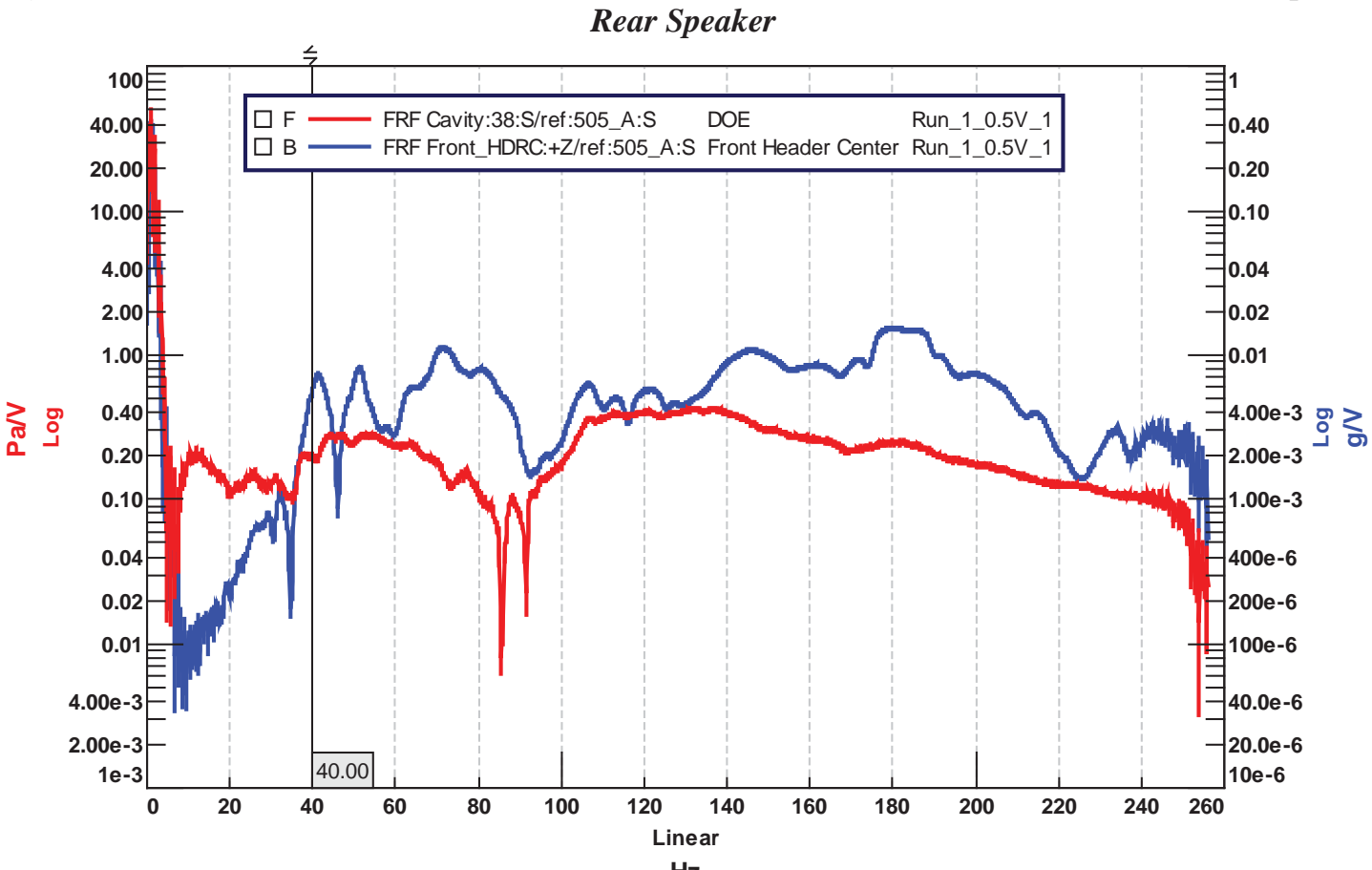

Figure 48: Acoustic FRF at DOE and Vibration FRF and Front Header Center with Acoustic Excitation at Front Speaker

Figure 49 shows the structural as well as acoustic response at the front header due to acoustic input due to the rear speaker. 
A clear peak is seen at $40 \mathrm{~Hz}$ for both responses indicating that the front header center has a strong participation in the boom frequency range.

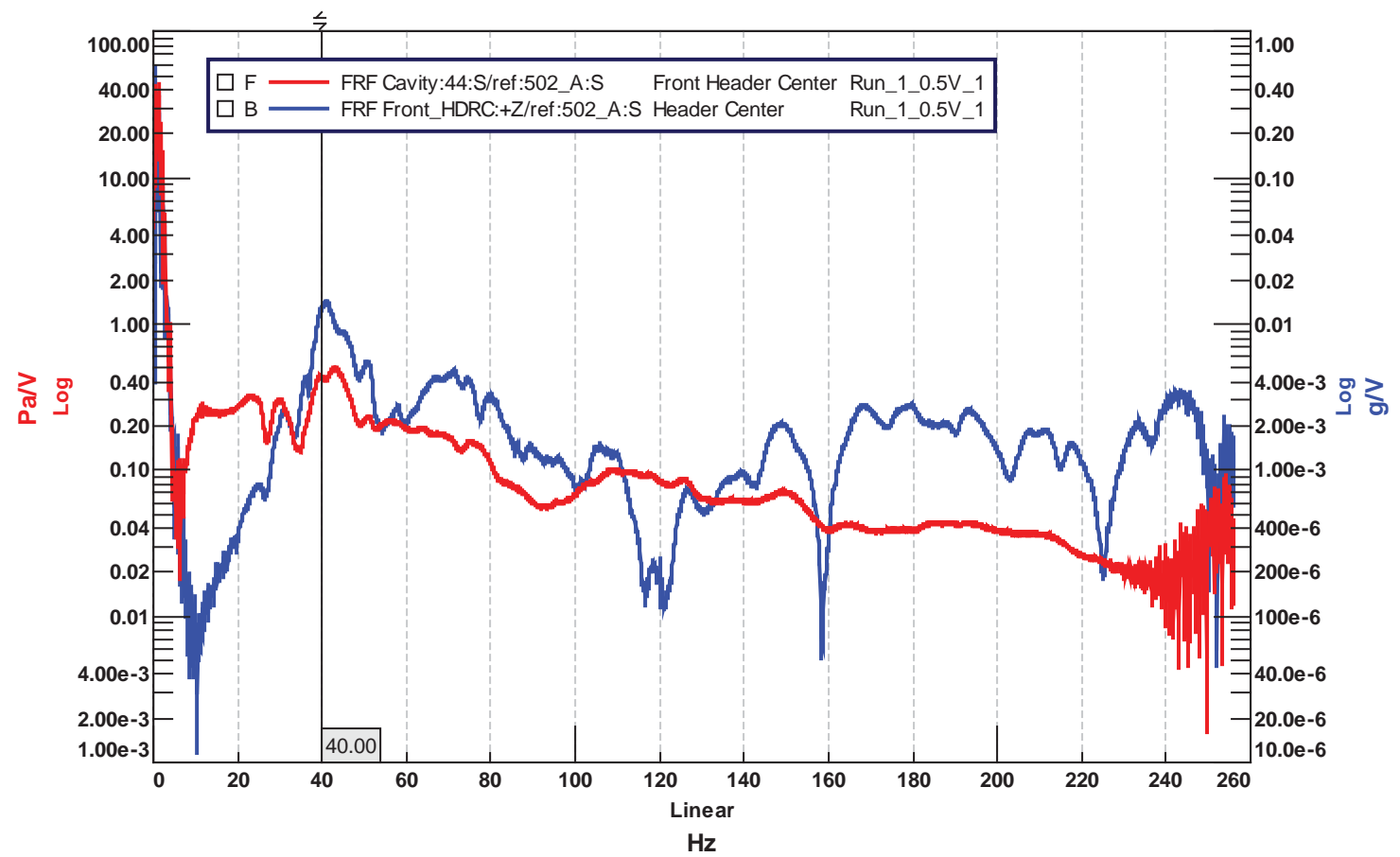

Figure 49: Acoustic FRF below Front Header Center and Vibration FRF at Front Header Center due to Rear Speaker

Figure 50 shows the structural and acoustic response at the rear header due to input from the rear speaker. The structural response shows a peak at $47 \mathrm{~Hz}$ which corresponds to the rear header mode. Acoustic response is also seen, however, it is not as strong as the structural response. This also indicates high participation from the rear header at $47 \mathrm{~Hz}$. It can also be concluded from the figure below that the boom is mainly felt due to the structure driving the vehicle fluid. An acoustic mode does not lie in the $40 \mathrm{~Hz}$ range. However, modes lying above or below this frequency may participate in the boom frequency range. It is also interesting to see the rear header structural response shows many peaks higher in the frequency range as well. Also, below $37 \mathrm{~Hz}$, the structural response is very low. As soon as the $40 \mathrm{~Hz}$ region is approached there is a sharp increase in the structural response ( $\sim 15$ times). This high structural response continues till about $80 \mathrm{~Hz}$. This high response may be reduced by using a tuned mass damper in the $40 \mathrm{~Hz}$ range. This would theoretically create anti-resonance in the $40 \mathrm{~Hz}$ range while creating side band peaks which have comparatively much lower amplitude than the original peak at $40 \mathrm{~Hz}$. 


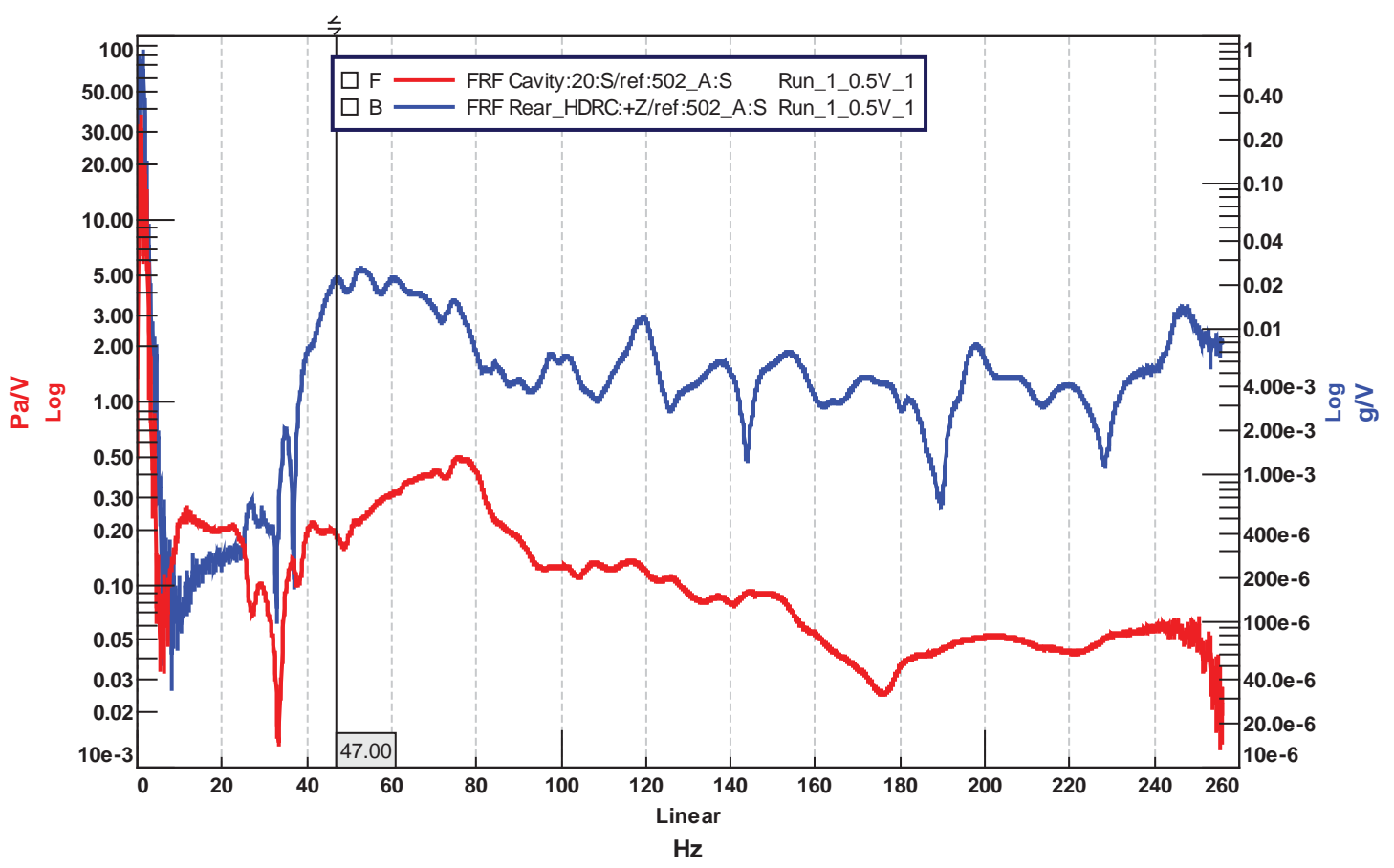

Figure 50: Acoustic FRF below Rear Header Center and Vibration FRF at Rear Header Center due to Rear Speaker

Figure 51 shows the acoustic mode at $39 \mathrm{~Hz}$ due to speaker inputs. According to this mode shape, it is mainly the trunk that shows high fluctuations in the acoustic pressure.

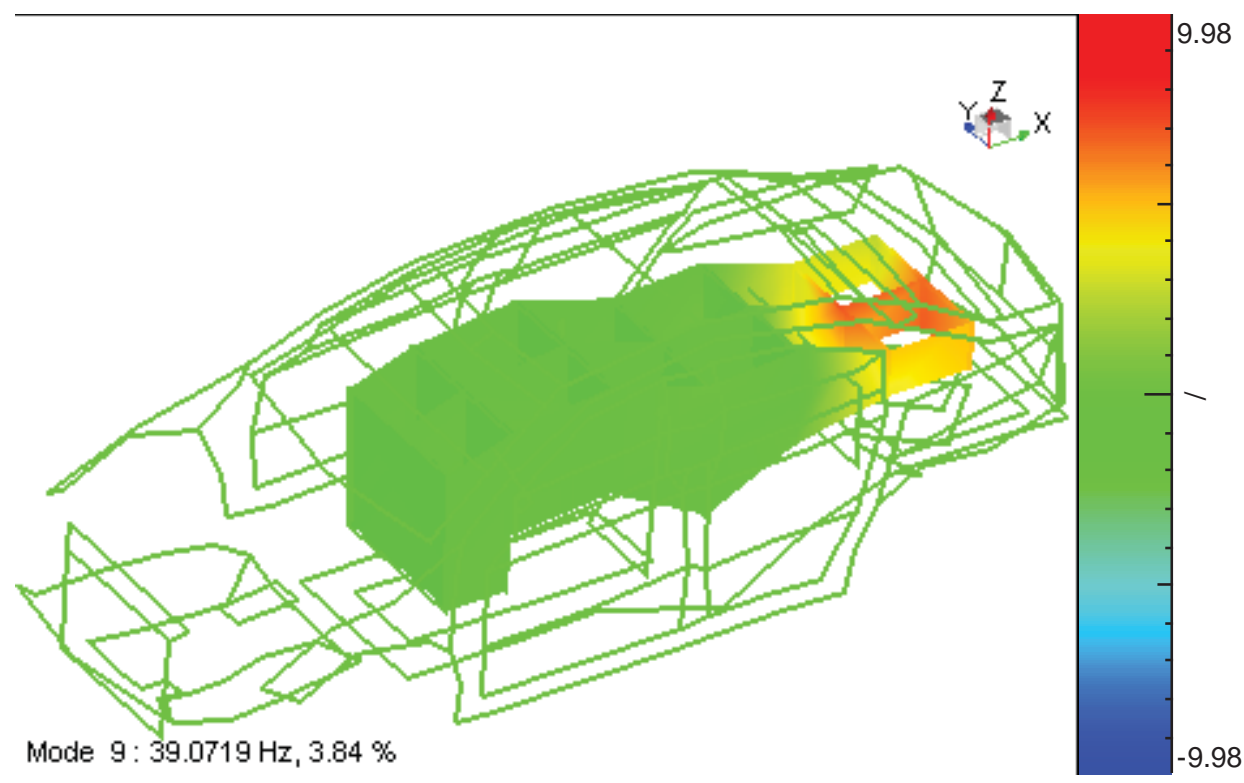

Figure 51: Acoustic Mode Shape at $39 \mathrm{~Hz}$ due to Speaker Inputs 
Figure 52 shows the acoustic mode shape at $41 \mathrm{~Hz}$. The pressure fluctuations occur mainly in the rear seats region and the trunk.

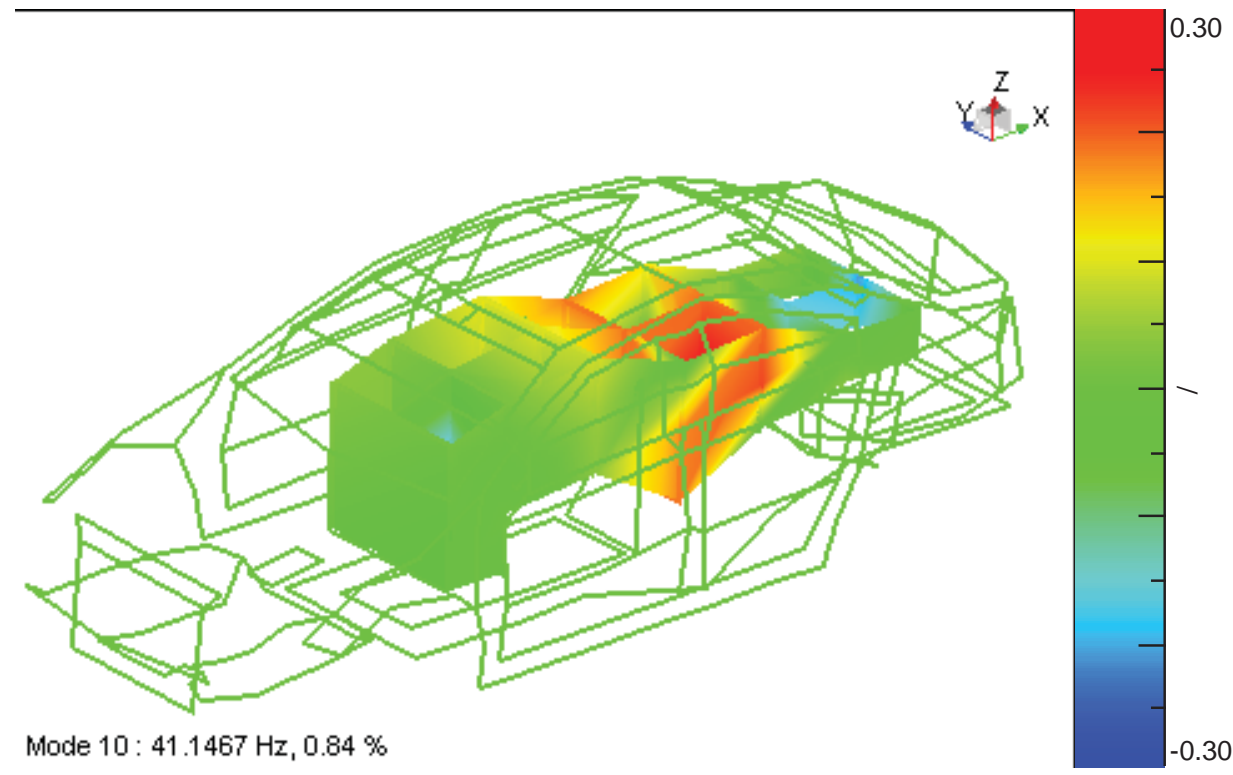

Figure 52: Acoustic Mode Shape at $41.14 \mathrm{~Hz}$ due to Speaker Inputs

Figure 53 shows the acoustic mode at $42.7 \mathrm{~Hz}$. This mode shapes shows pressure fluctuations primarily in the trunk and secondarily in the front seats. This mode shape resembles the boom mode of interest as the pressure fluctuations are also present in the front seats region.

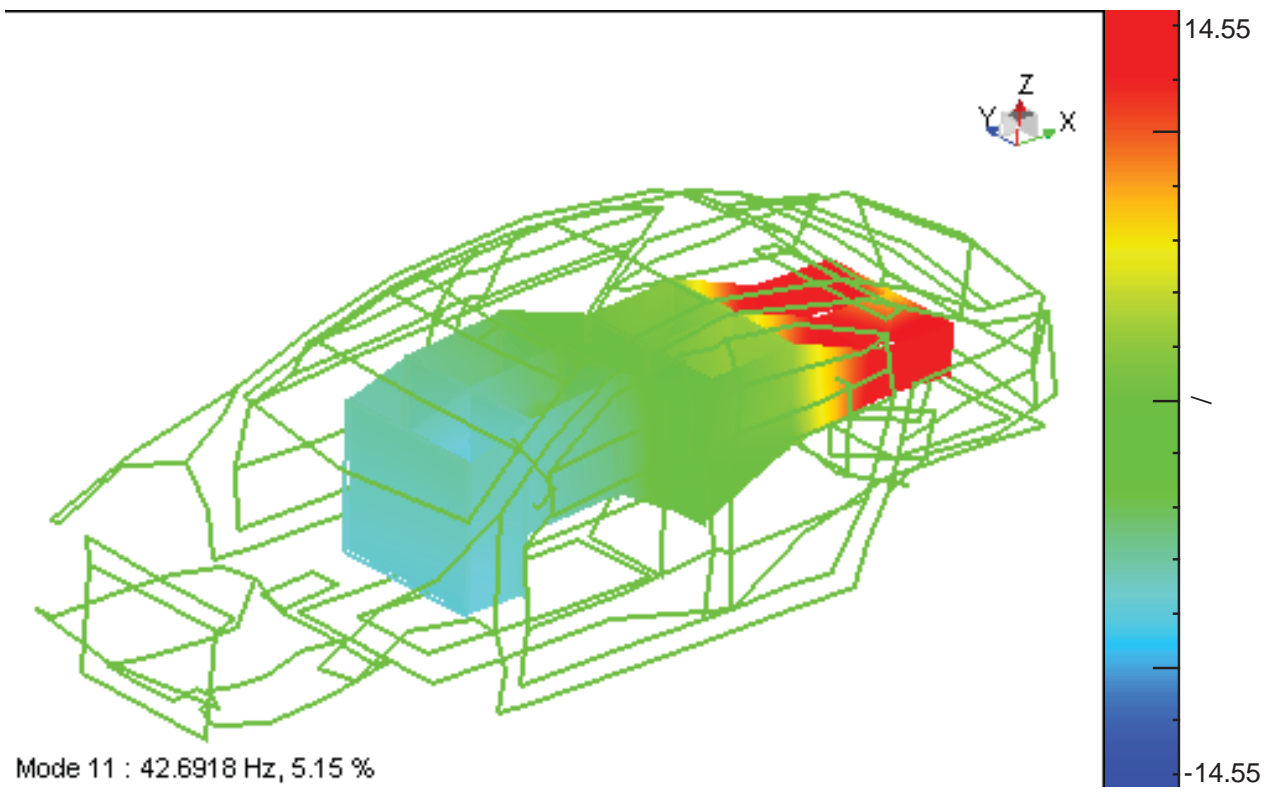

Figure 53: Acoustic Mode Shape at $42.7 \mathrm{~Hz}$ due to Speaker Inputs 
Figure 54 shows the acoustic mode shape at $46.3 \mathrm{~Hz}$. This is the rear header mode. The pressure fluctuations are in the rear seats region with almost no fluctuation in the rest of the cavity.

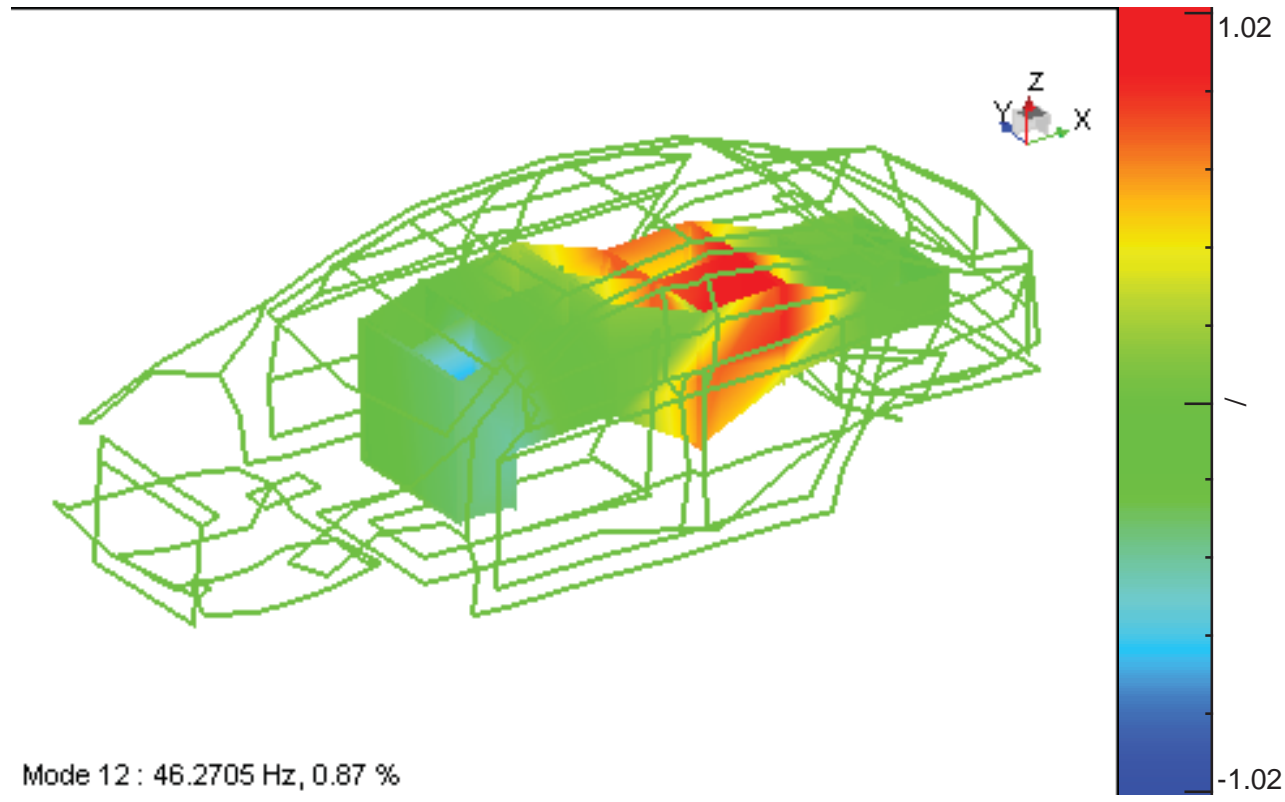

Figure 54: Acoustic mode shape at $46.3 \mathrm{~Hz}$

Figure 55 shows the Modal Assurance Criterion (MAC) for the acoustic degrees of freedom (DOFs) between mode shapes when the structure was excited with the help of shakers and when it was excited with the help of speakers. The MAC is shown up to 47 $\mathrm{Hz}$ which covers the boom frequency region. In the case of speaker excitation, mainly, the acoustic modes get excited. Hence we will know which acoustic modes participate in the boom range when the structure is excited by shakers.

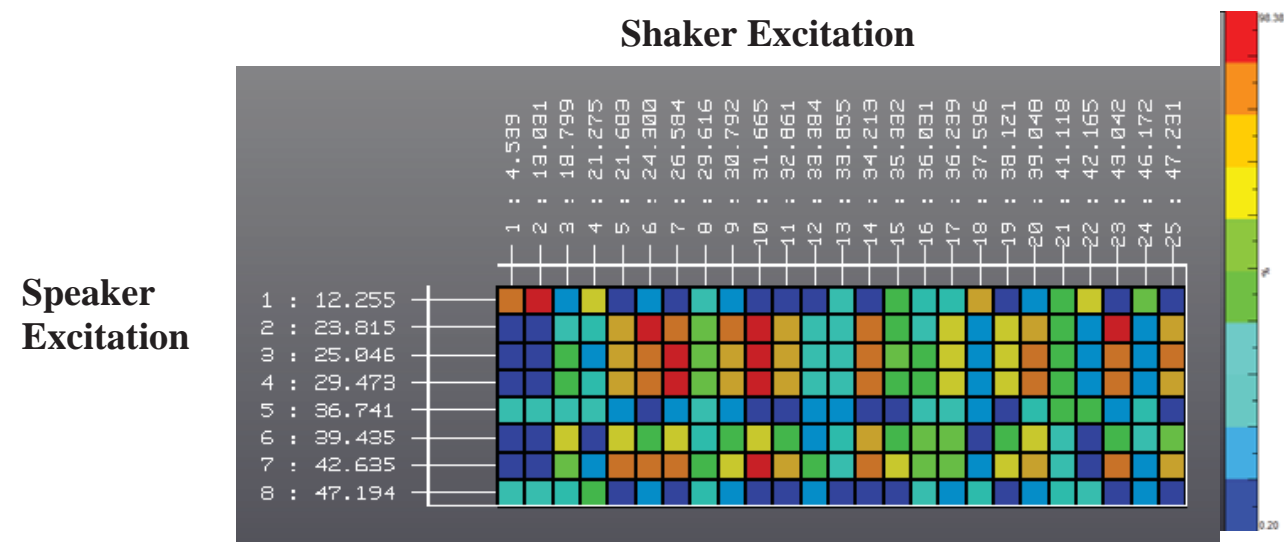

Figure 55: MAC for Acoustic DOFs with Shaker and Speaker Excitation 
It can be seen from the MAC plot that many acoustic modes participate with the shaker excitation modes. For example, for the shaker excitation mode at $31.6 \mathrm{~Hz}$, there is strong participation from three acoustic modes- $23.8 \mathrm{~Hz}, 25 \mathrm{~Hz}$ and $29.47 \mathrm{~Hz}$. In the $40 \mathrm{~Hz}$ boom range, the participation is mainly from the $25 \mathrm{~Hz}$ and $29 \mathrm{~Hz}$ acoustic modes. At 47 $\mathrm{Hz}$ where there is participation from the rear header, the $25 \mathrm{~Hz}$ acoustic mode shows good participation. The $25 \mathrm{~Hz}$ acoustic mode is shown in Figure 56. It is basically the first acoustic mode where there are alternate pressure fluctuations in the vehicle cabin and trunk of the vehicle. The acoustic modes at $23 \mathrm{~Hz}$ and $29 \mathrm{~Hz}$ are similar to this mode as well.

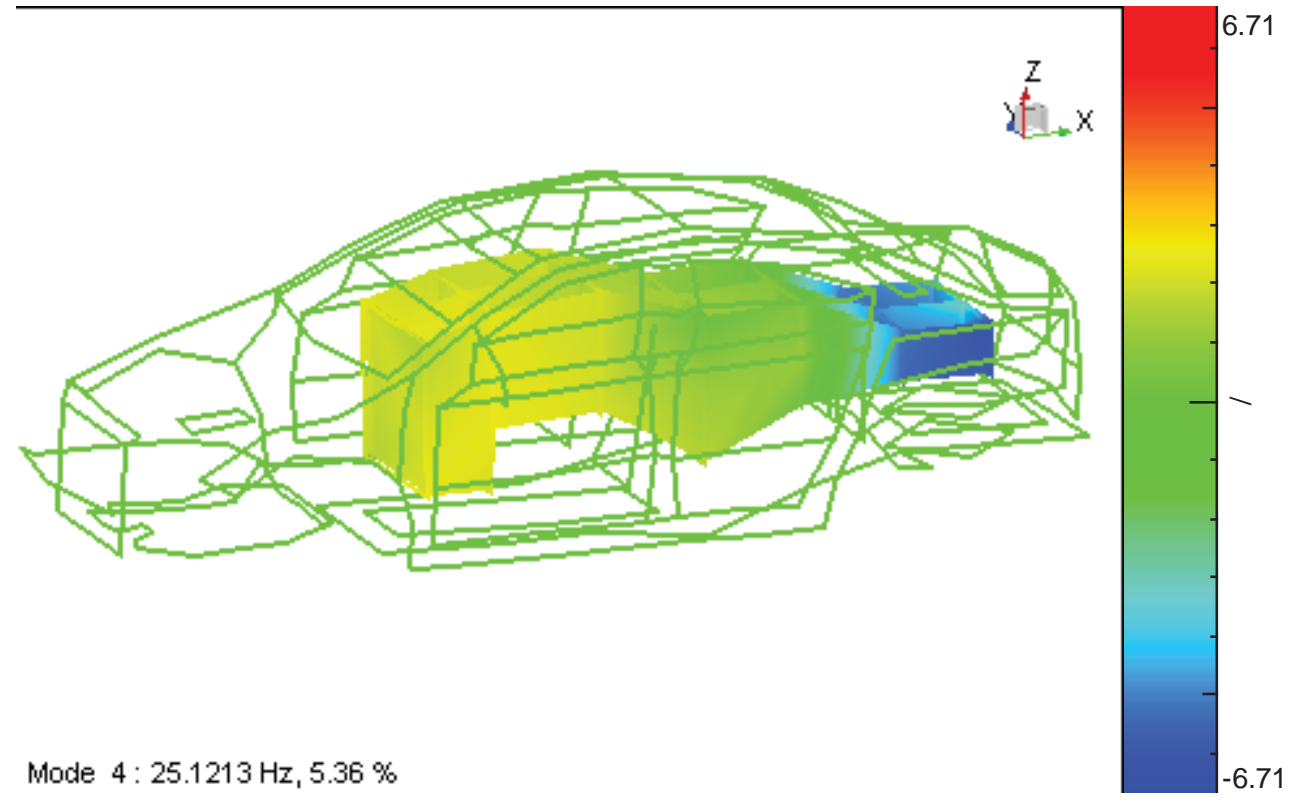

Figure 56: Acoustic Mode at $25.1 \mathrm{~Hz}$

\subsection{Summary of Acoustic Modal Test with Acoustic Excitation}

With the help of this test crucial information about the behavior of the structure with acoustic inputs is obtained. This test confirms that there is fluid structure interaction or vibro-acoustic coupling present in the vehicle. Furthermore, this test helps to identify the acoustic modes of the vehicle cavity and compute their interaction with the modes due to shaker input by the Modal Assurance Criterion. It is observed that there are multiple acoustic modes that interact in the boom range. The primary modes that interact in the boom range are similar to the first acoustic mode where there are alternate pressure fluctuations in the vehicle cabin and the trunk of the car. 


\section{Full Acoustic Modal Test - Structural Input: Door Cavity Testing}

\subsection{Purpose}

Along with modal testing of the vehicle, Finite Element Analysis of its model was being performed simultaneously [6]. It was thought that the door cavities might behave like Helmholtz resonators and make the vehicle cavity effectively longer. This would not only shift the peaks of the acoustic modes but also introduce new modes for the door cavities. Based on the resonator effect, the acoustic response at the driver's right ear (DRE) might be reduced. This prompted the Door Cavity testing in order to confirm the above mentioned hypothesis.

\subsection{Description}

There were four different configurations in which the door cavity testing took place:

- With original production door panels

- With the original production door panels removed

- With half inch Oriented Strand Board (OSB) sheets covering the door cavities with an access for speaker hole

- With half inch OSB sheets completely covering the door cavities (access hole for speaker blocked)

Initial testing with the production door panels would reveal the behavior of the door panels as to whether they act as a sound barrier or a sound absorber, or in a worst case scenario if they are acoustically transparent. This was important as the structural testing had confirmed contribution from the doors. Hence if the outer panel of the doors would deform, it would create acoustic pressure in the door cavities which would then transfer to the vehicle cabin. The door panels were then removed to understand the effect of making the vehicle cavity acoustically longer. Half inch OSB sheets were then used to cover the door cavities as they would behave as an acoustic barrier and would not let the acoustic fluctuations inside the door cavity get transmitted inside the vehicle cabin. An access hole for the speaker was left open to simulate the actual behavior of the speaker in normal conditions. The speaker is acoustically transparent and would let the acoustic fluctuations pass through. A final configuration where the entire door cavity was blocked with OSB sheets was also tested to observe the effect of completely isolating the door cavities from the main cavity.

The importance of modeling the door cavities in the Finite Element Model was to be confirmed by physical testing. If in the testing, it was to be found that the door cavities are not significant for the boom effect, then it would not be necessary to model and solve for them making the process a little bit quicker and easier as well. 
Figure 57 shows the door cavities when the production door panels are completely removed.
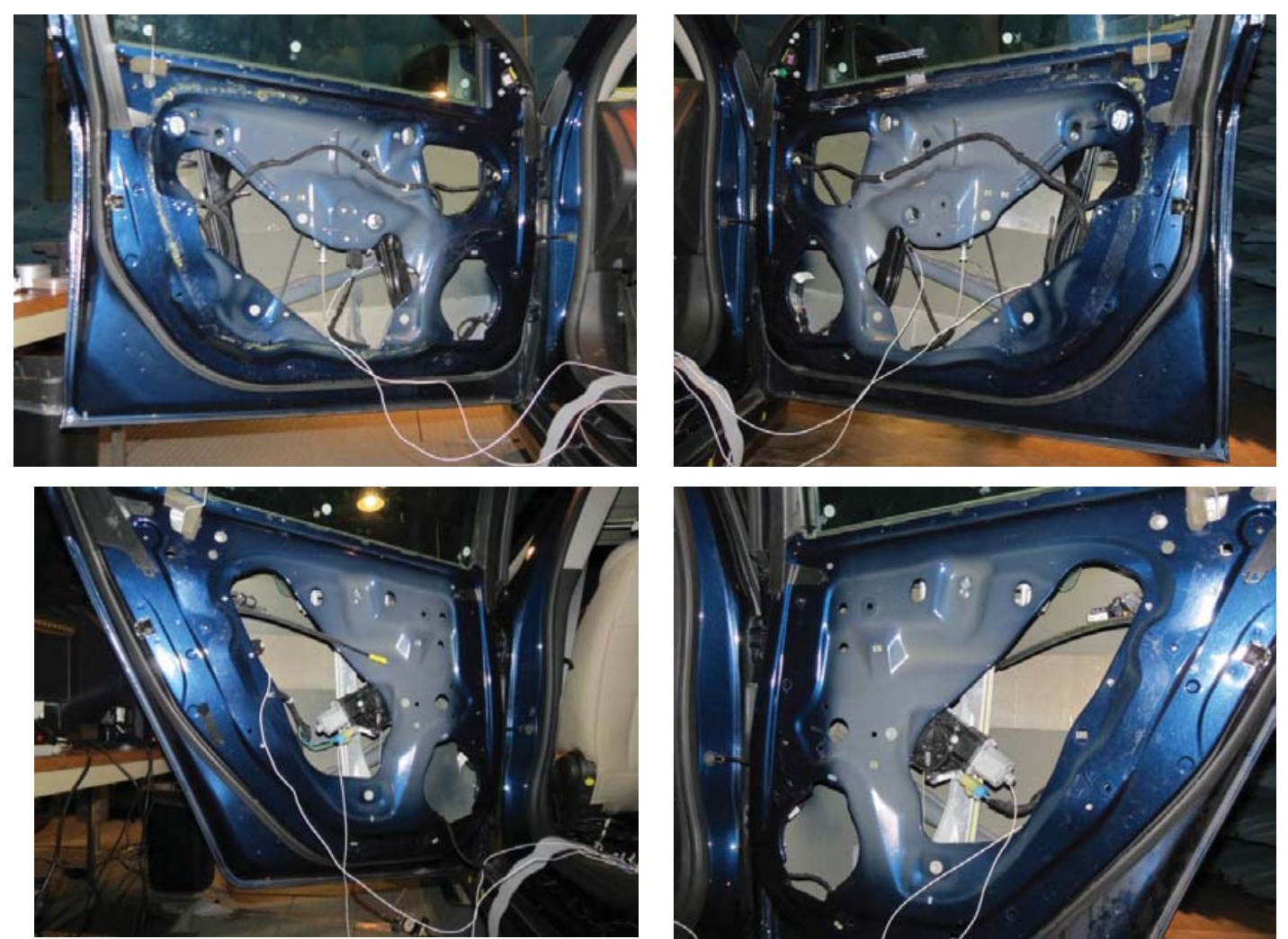

Figure 57: Chevrolet Cruze with Door Panels Removed, Clockwise: Driver Door, Front Passenger Door, Rear Door behind Front Passenger, and Rear Driver behind Driver

Figure 58 shows the microphone locations inside the door cavities on the driver side doors. Two microphones were used for each door cavity in order to evaluate any difference in acoustic pressure. The microphones were mounted on the door stiffeners in the cavity.

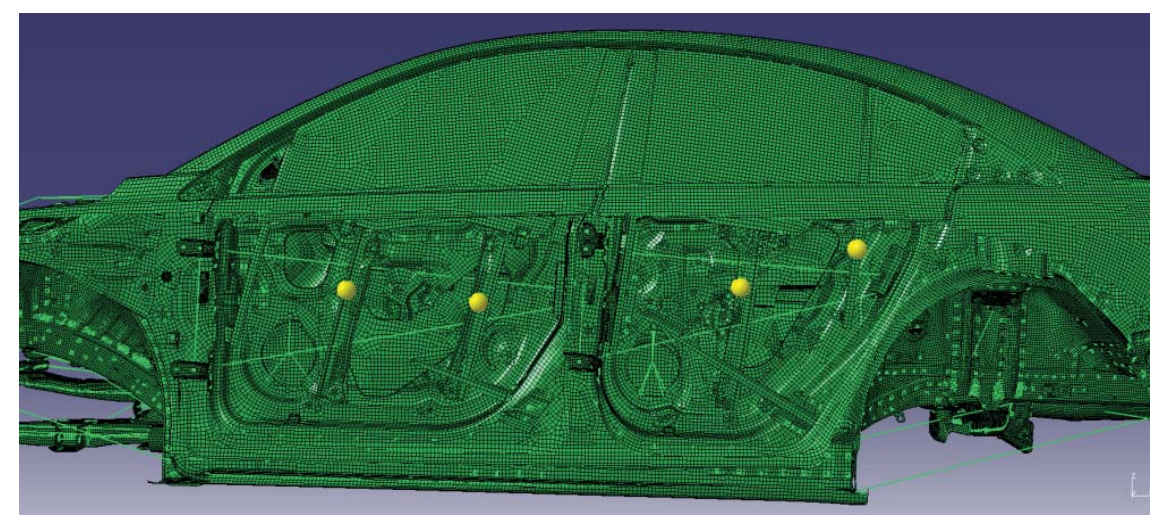

Figure 58: Microphone locations in Door Cavities (Driver Side) 
Figure 59 shows the microphone locations for the door cavities on the passenger side.

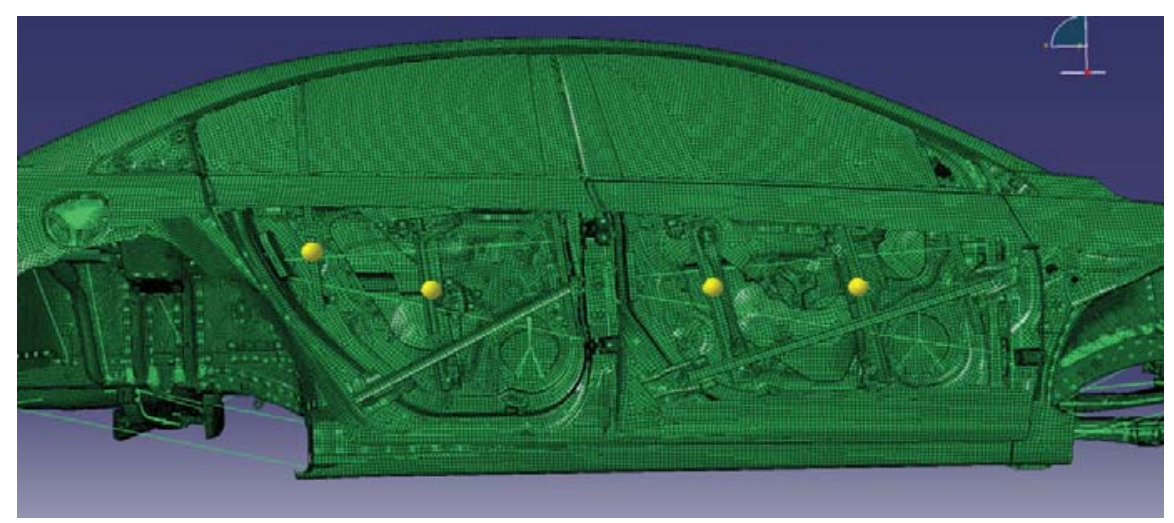

Figure 59: Microphone locations in Door Cavities (Passenger Side)

Figure 60, Figure 61, Figure 62, and Figure 63 show the application of half inch OSB sheets to seal the door cavities for the driver door, front passenger door, rear passenger door behind front passenger, and rear passenger door behind driver respectively. An access for the speaker opening is kept open and tested first. Later, it is closed and tested again. As the contour of the door cavities was not exactly flat, air gaps existed which had to be sealed with the help of fiber glass, vinyl foam and duct tape. Hence, though theoretically the OSB sheets were supposed to seal off the door cavities, due to the use of fiber glass and other materials, some of the acoustic pressure fluctuations may have leaked in the vehicle cabin. The exact amount cannot be ascertained, however, for the purpose of these tests, it was assumed that the door cavities were completely sealed.

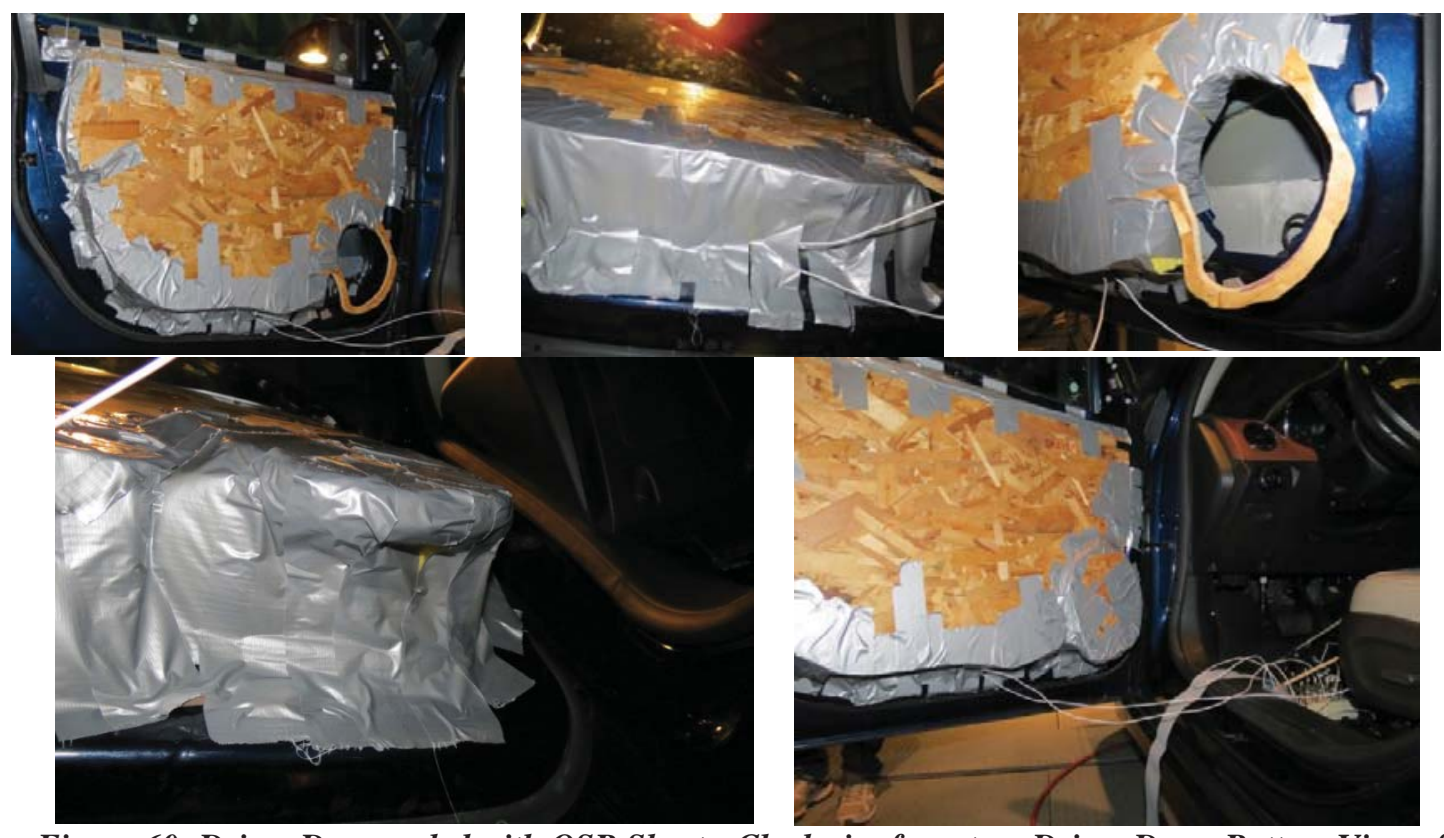

Figure 60: Driver Door sealed with OSB Sheets. Clockwise from top: Driver Door, Bottom View of Driver Door, Access for Speaker hole, Driver Door with no Access, and No Access Bottom View 

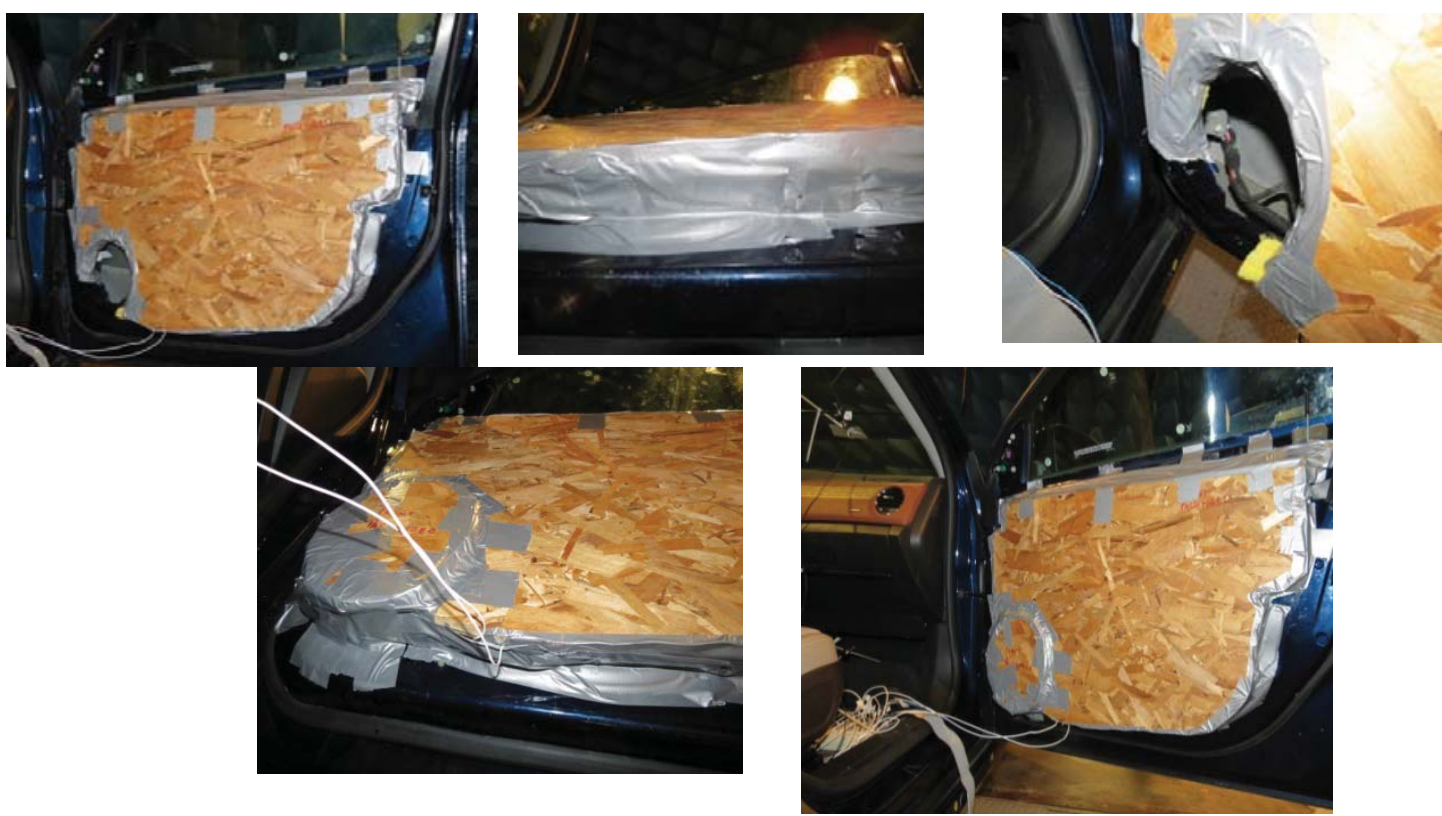

Figure 61: Front Passenger Door sealed with OSB Sheets. Clockwise from top: Front Passenger Door, Bottom View of Front Passenger Door, Access for Speaker hole, Front Passenger Door with no Access, and No Access Bottom View

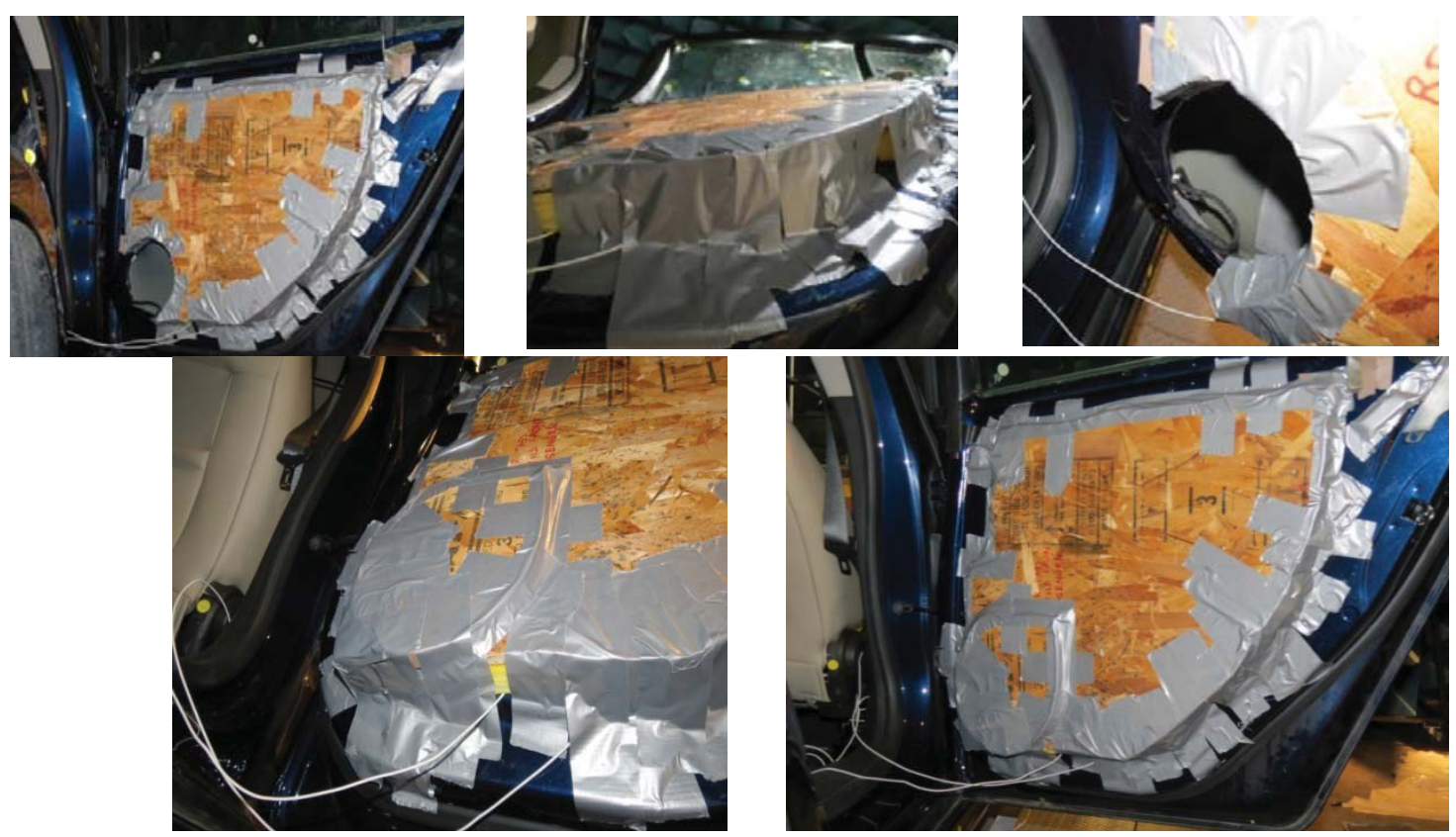

Figure 62: Rear Passenger Door behind Front Passenger sealed with OSB Sheets. Clockwise from top: Rear Passenger Door, Bottom View of Rear Passenger Door, Access for Speaker hole, Rear Passenger Door with no Access, and No Access Bottom View 

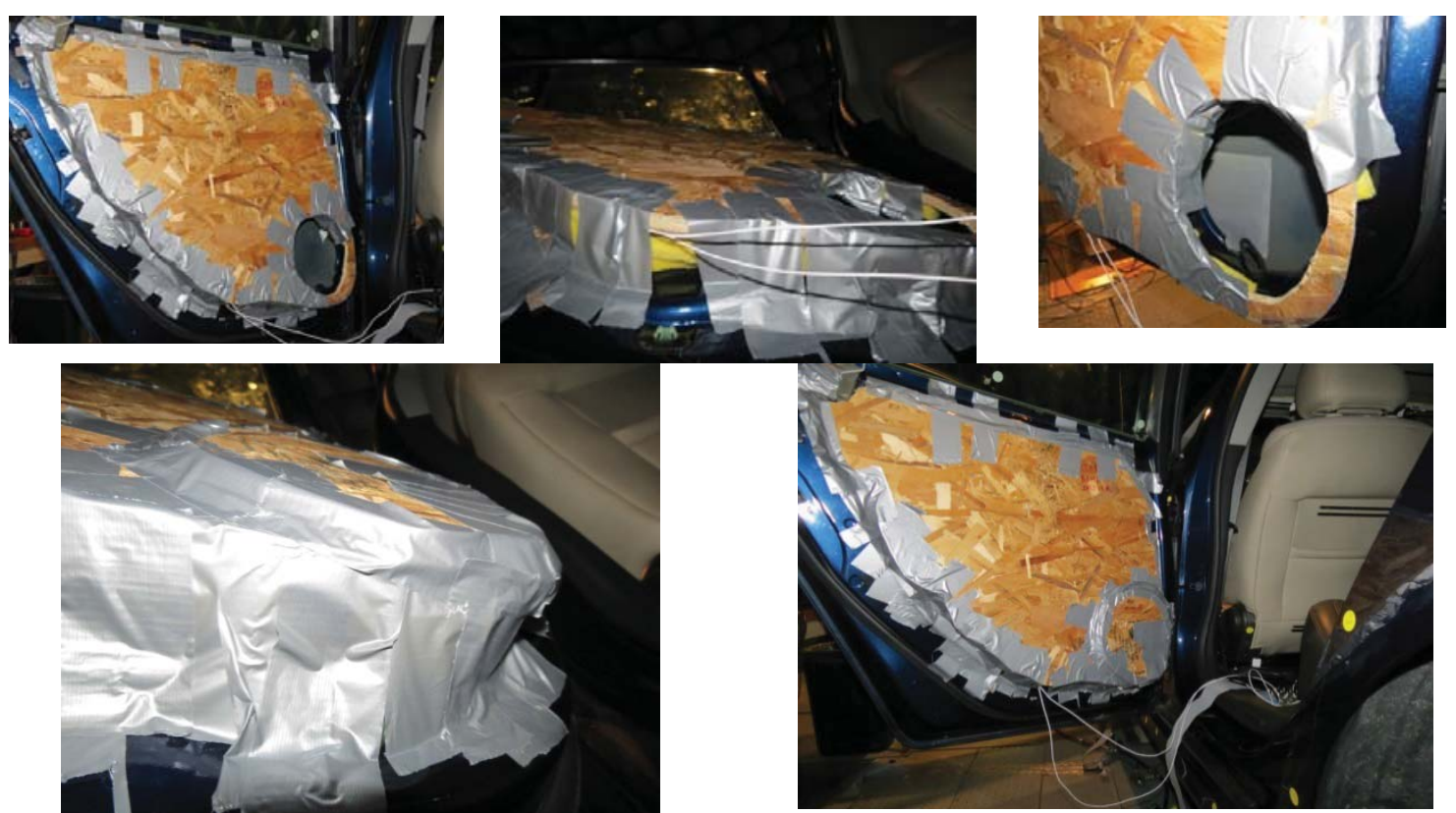

Figure 63: Rear Passenger Door behind Driver sealed with OSB Sheets. Clockwise from top: Rear Passenger Door, Bottom View of Rear Passenger Door, Access for Speaker hole, Rear Passenger Door with no Access, and No Access Bottom View

Figure 64 shows the acoustic response at DRE due to the rear shaker. It can be clearly seen from the plot that the different configurations of door cavity testing do not have an effect in the $40 \mathrm{~Hz}$ boom range. The effect is seen at other frequencies, for example in the $33 \mathrm{~Hz}$ range and in the $55 \mathrm{~Hz}$ range.

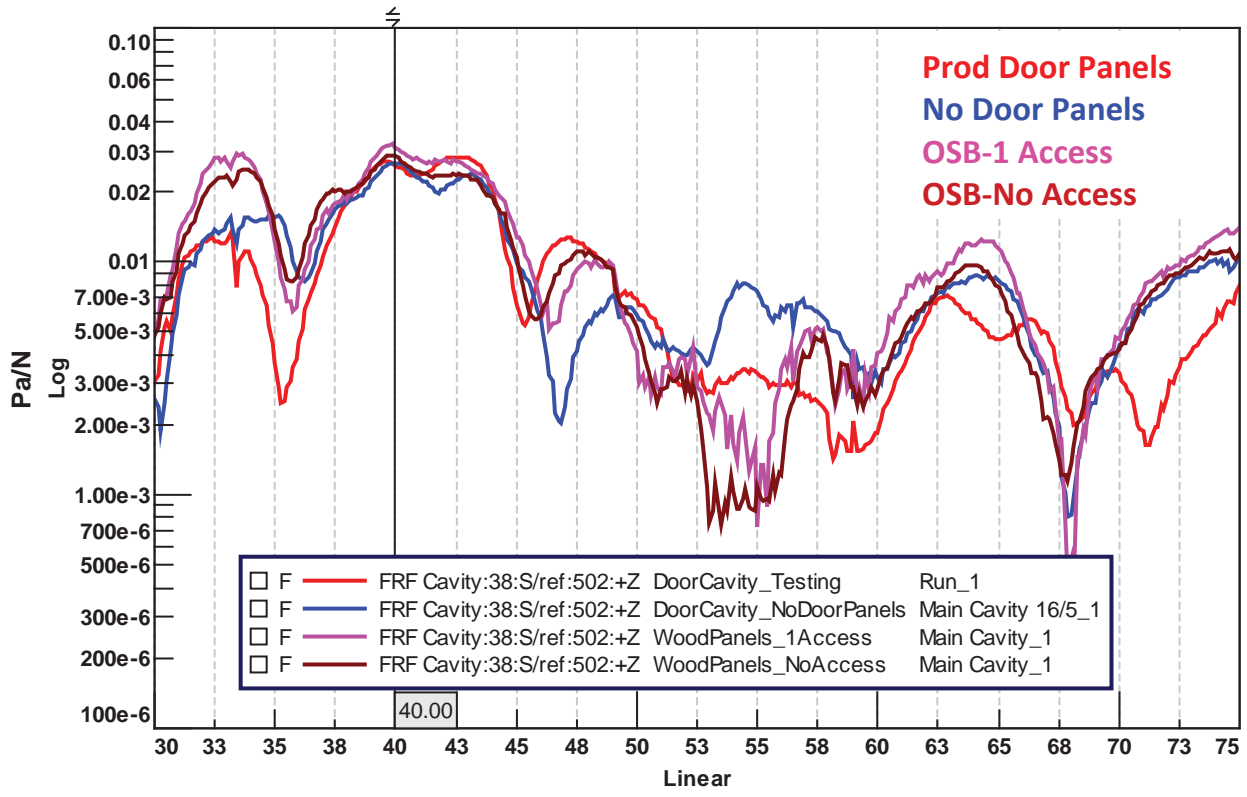

$\mathrm{Hz}$

Figure 64: Acoustic FRF at DRE due to Rear Shaker for different Door Configurations 
Figure 65 shows the acoustic FRF at DRE due to the front shaker for the different door configurations. In this case, a difference is seen in the $40 \mathrm{~Hz}$ boom range. Testing with the production door panels shows the highest response followed by the OSB sheets with one access and no access. The lowest response is observed when the door cavities are completely open to the vehicle cabin. This is understandable as the volume of the door cavities get added to the main cavity, making the cavity acoustically longer. It is also interesting to see that at the DRE, there is no significant difference whether the OSB sheet completely seals off the door cavity or if one access is kept. The responses almost overlap.

Figure 66 shows the acoustic FRFs in the driver door cavity for the different configurations of testing. The response is lowest when the door cavities are completely open, while the response is generally the highest for the frequency range of $30-75 \mathrm{~Hz}$ when the door cavity is completely sealed by OSB sheets. The response due to production door panels is lower than the OSB sheets. No boom mode is observed at 40 $\mathrm{Hz}$ for any of the configurations.

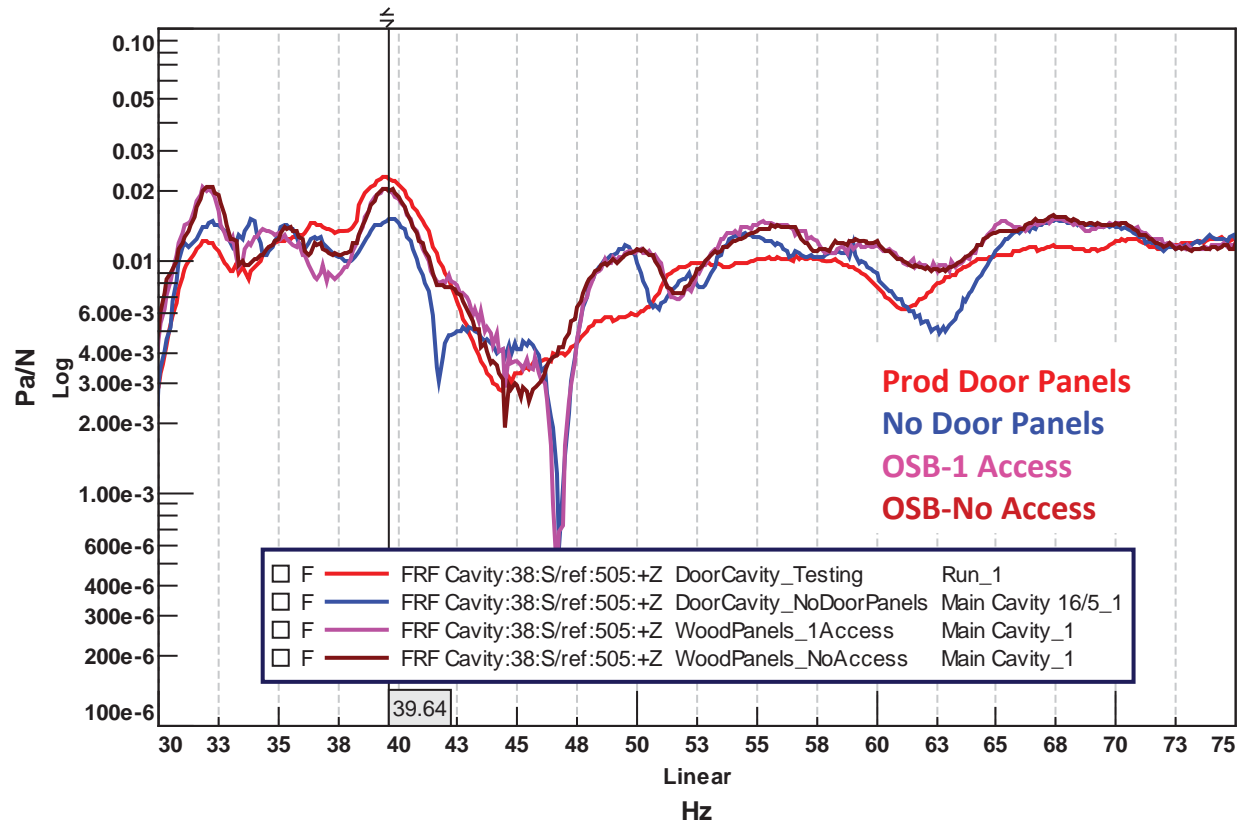

Figure 65: Acoustic FRF at DRE due to Front Shaker for different Door Configurations 


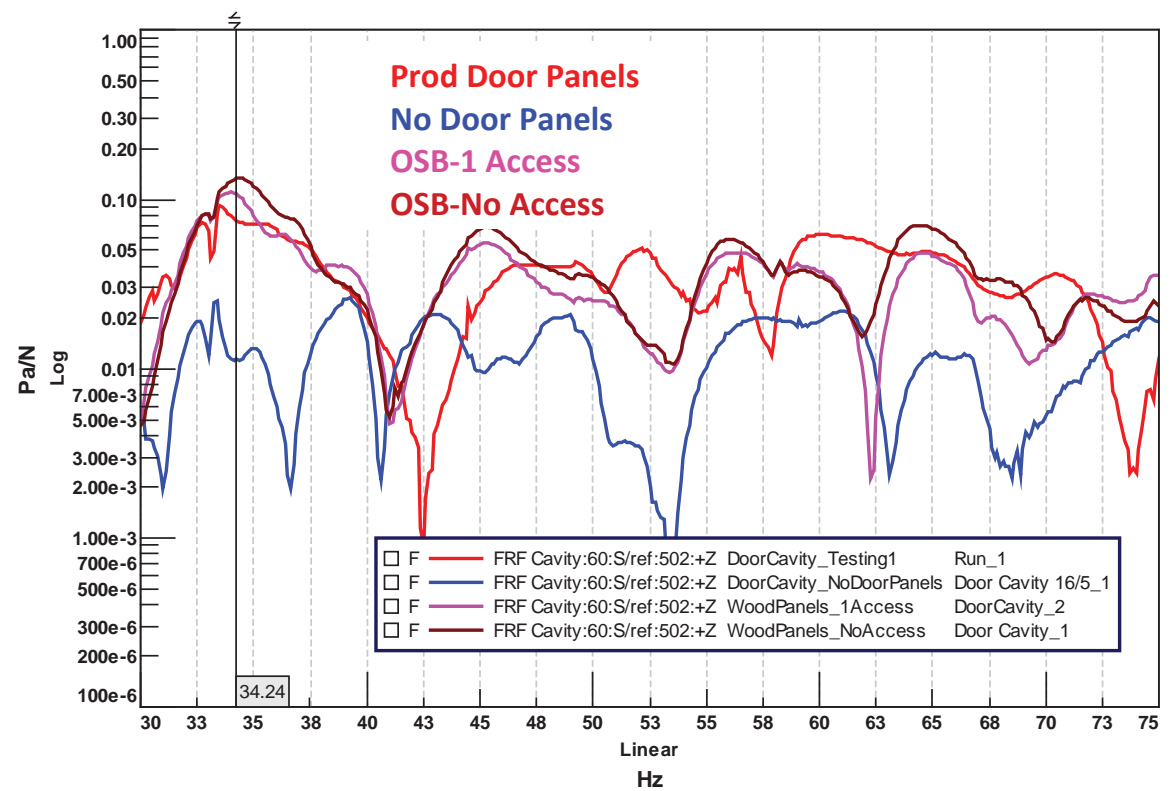

Figure 66: Acoustic Response in Driver Door Cavity due to Rear Shaker for different Door Configurations

Acoustic FRFs in the driver door cavity due to the front shaker are shown in Figure 67. Below $40 \mathrm{~Hz}$, the response due to production door panels is observed to be the lowest while the response with the OSB sheets is high. Peaks are seen at around $38 \mathrm{~Hz}$ for all the door configurations. It seems that the front shaker is exciting some modes in the boom range.

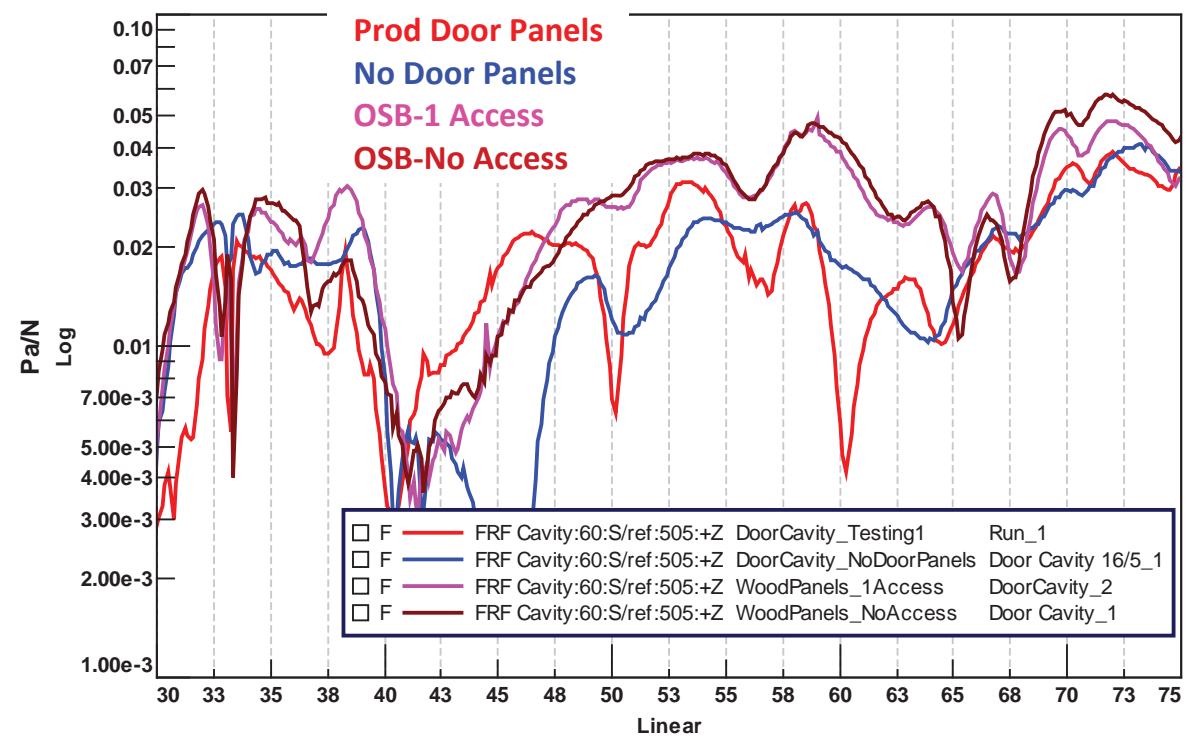

$\mathrm{Hz}$

Figure 67: Acoustic Response in Driver Door Cavity due to Front Shaker for different Door Configurations 
The response in the front passenger door cavity due to the rear shaker is shown in Figure 68. A peak is seen at $40 \mathrm{~Hz}$ when the vehicle is tested with no door panels. The peak is shifted lower in frequency, at around $37.5 \mathrm{~Hz}$ for the other configurations. Below the 40 $\mathrm{Hz}$ range, the response is higher when the OSB configurations are used. Above $40 \mathrm{~Hz}$, the response due to the production panels dominates the frequency range till $75 \mathrm{~Hz}$.

Figure 69 shows the response for the front passenger door cavity due to the front shaker. At $40 \mathrm{~Hz}$, the response due to the production door panels is the least; however, it shoots off for the 40-75 Hz range. For the configuration of when the door panels are completely removed, the response drops significantly for the range of $40 \mathrm{~Hz}-68 \mathrm{~Hz}$.

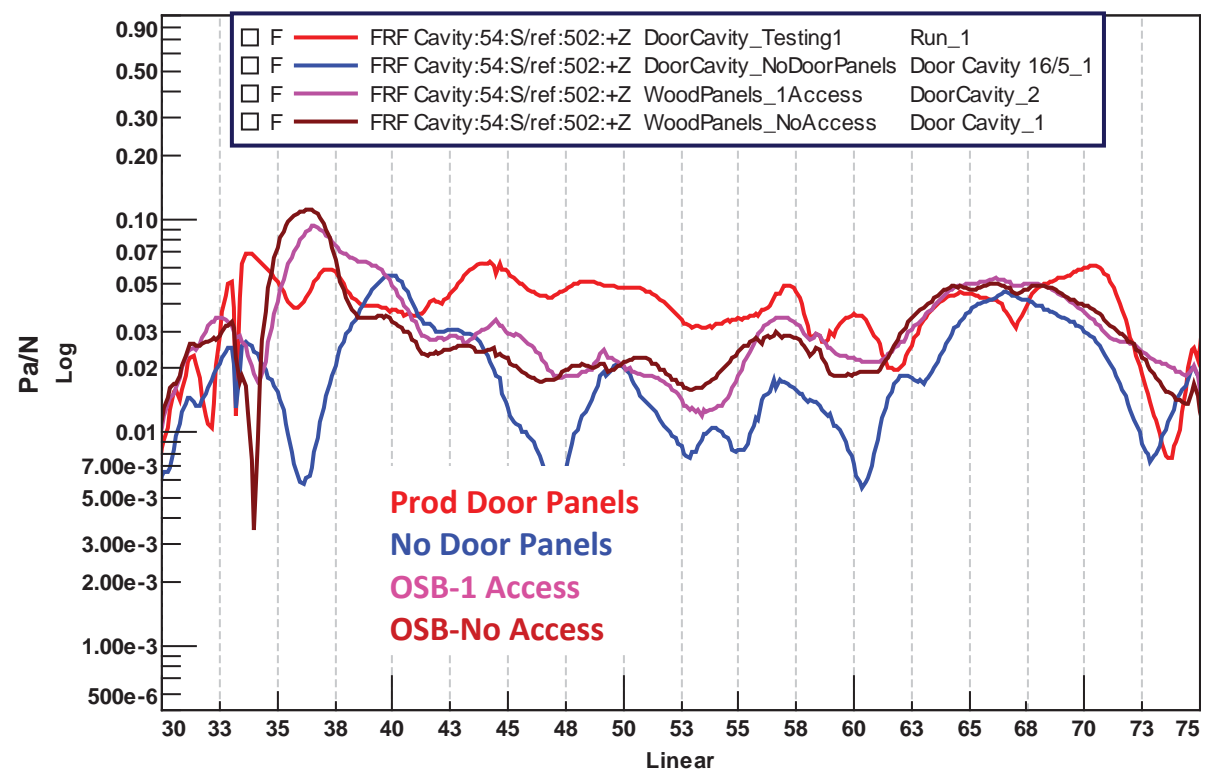

$\mathrm{Hz}$

Figure 68: Acoustic Response in Front Passenger Door Cavity due to Rear Shaker for different Door Configurations 


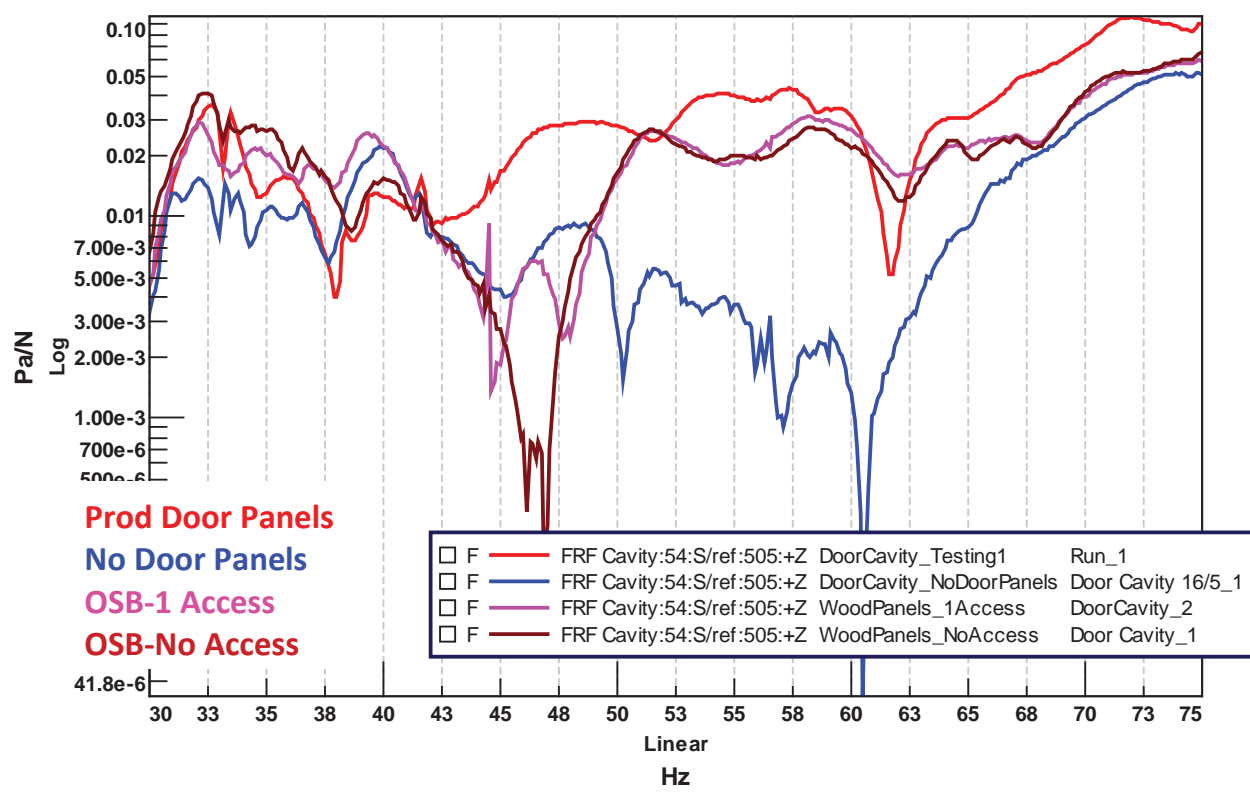

Figure 69: Acoustic Response in Front Passenger Door Cavity due to Front Shaker for different Door Configurations

Figure 70 shows the acoustic FRF in the rear passenger door cavity behind the driver due to rear shaker. The response due to the production door panels is the highest in the $40 \mathrm{~Hz}$ boom range.

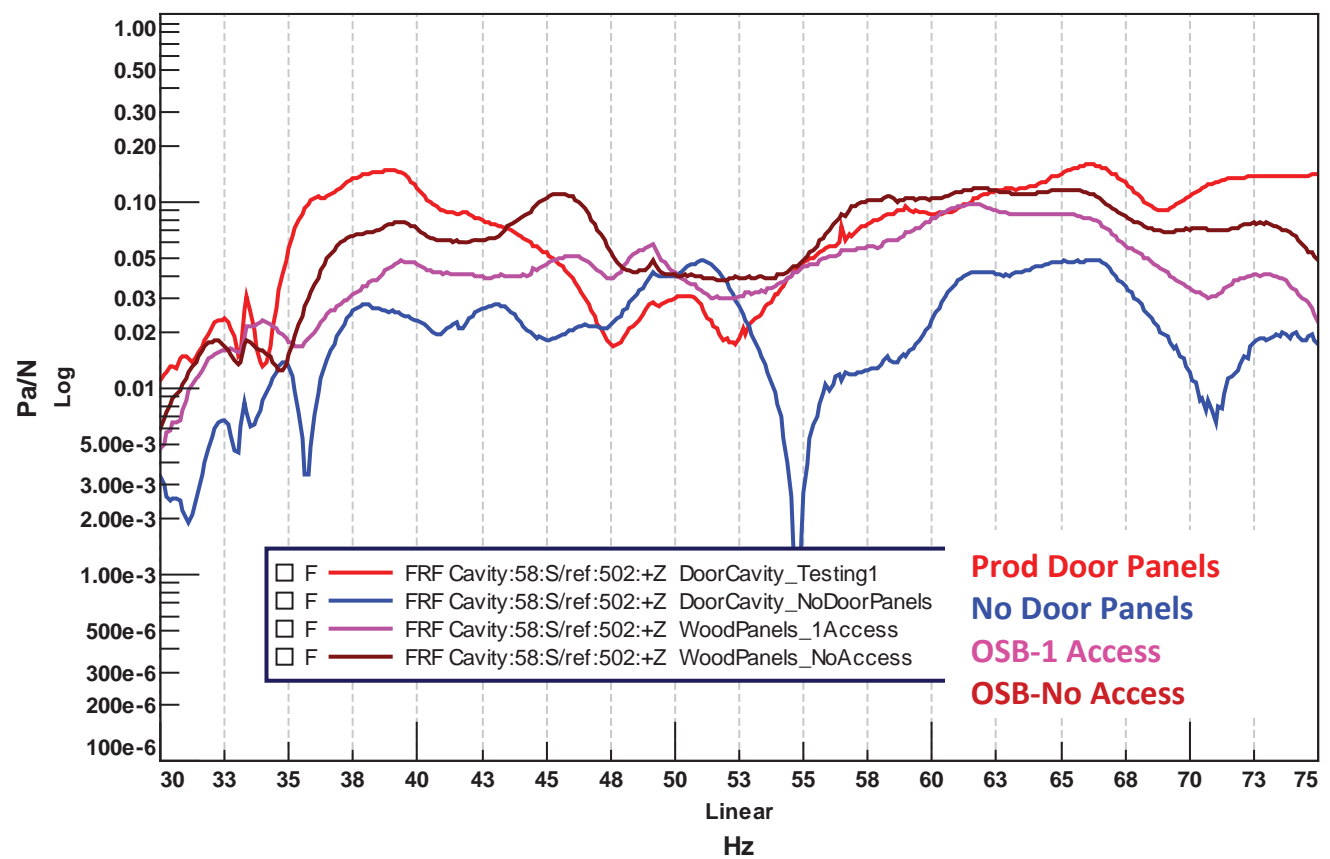

Figure 70: Acoustic Response in Rear Passenger Door Cavity behind Driver due to Rear Shaker for different Door Configurations 
Figure 71 shows the response due to force input from the front shaker. The trend for all the configurations is similar for this door cavity. Peak is in the $40 \mathrm{~Hz}$ range for all the configurations except for the production door panels. The response due to the production door panel configuration shows a lot of variation.

Figure 72 shows the response at the rear passenger door cavity behind the front passenger due to rear shaker. The response of the production door panels' configuration dominates the 33-43 Hz frequency range and also $55-75 \mathrm{~Hz}$ range. As seen earlier, the response for the no door panel configuration is the lowest as there is no surface to reflect the sound waves.

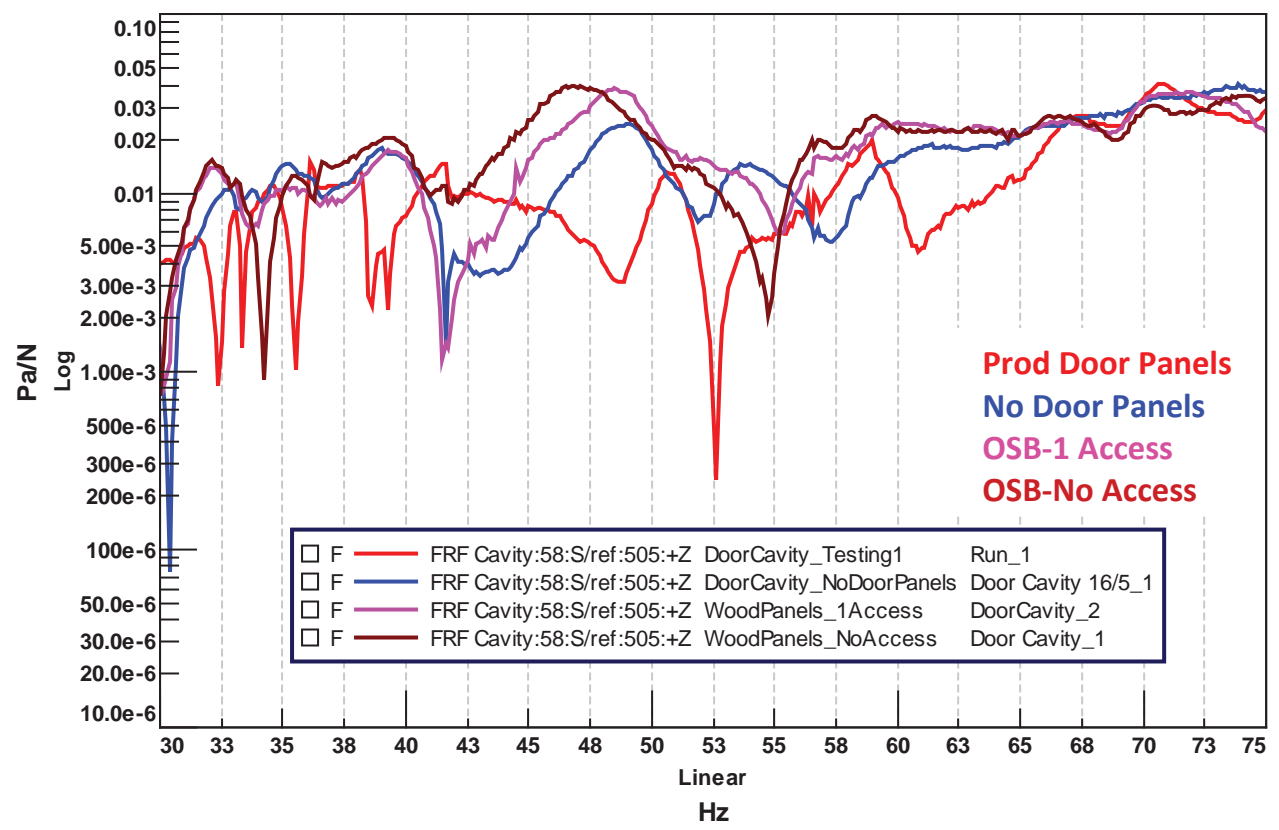

Figure 71: Acoustic Response in Rear Passenger Door Cavity behind Driver due to Front Shaker for different Door Configurations 


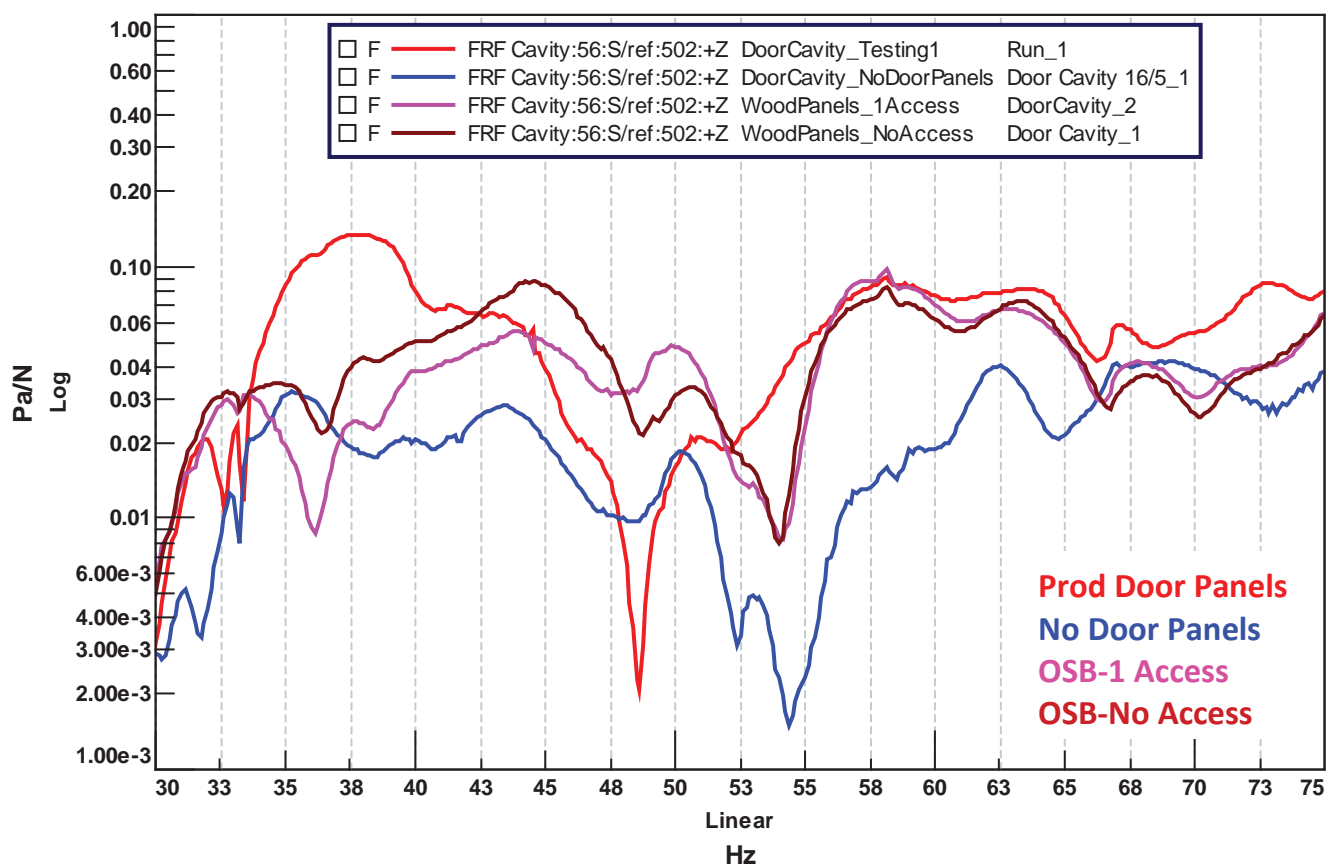

Figure 72: Acoustic Response in Rear Passenger Door Cavity behind Front Passenger due to Rear Shaker for different Door Configurations

Figure 73 shows the acoustic FRF at the rear passenger door cavity behind the drive due to force inputs from the front shaker. In this case as well, in the $40 \mathrm{~Hz}$ boom range the response due to the production panels is the highest.

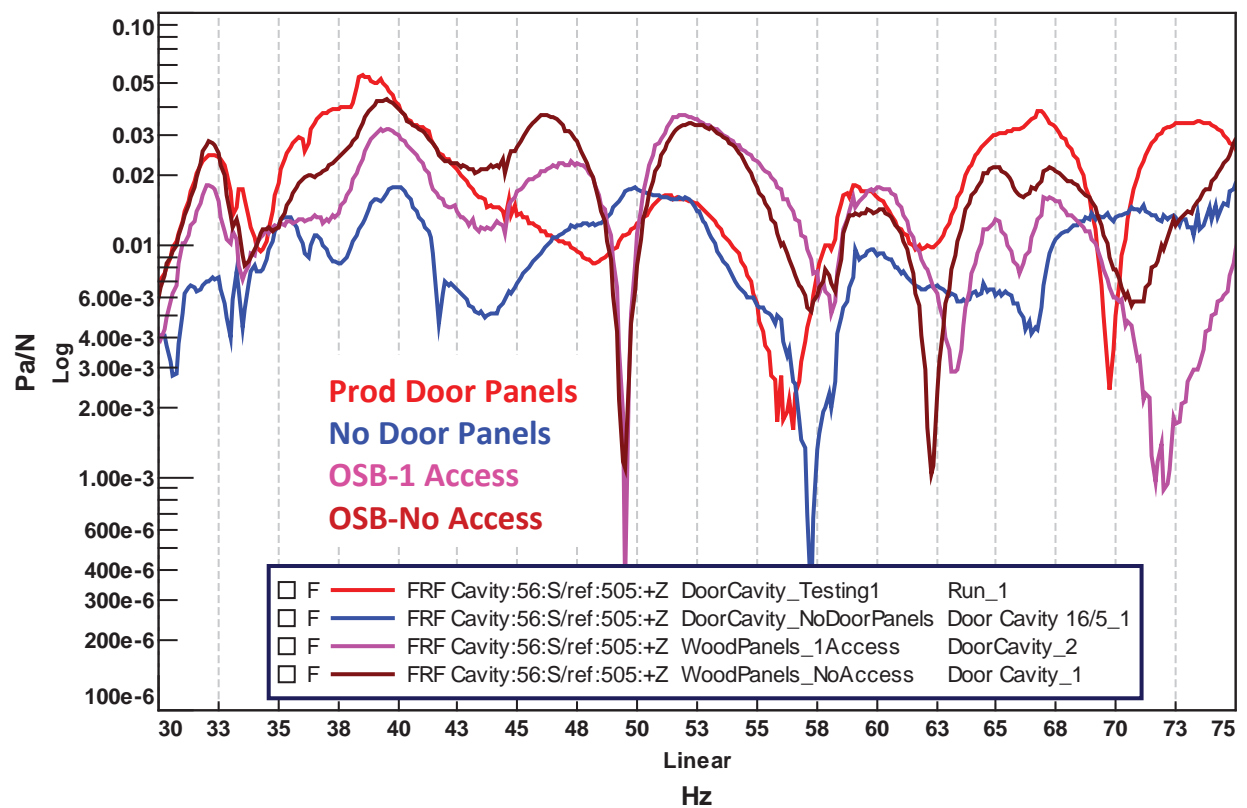

Figure 73: Acoustic Response in Rear Passenger Door Cavity behind Front Passenger due to Front Shaker for different Door Configurations 
So far we have seen that the response in the door cavities changes depending upon the source of excitation. The rear shaker does not excite the mode in the $40 \mathrm{~Hz}$ range but the front shaker does. The front shaker is located below the front passenger seat.

It would also help to understand the response of the door panels to along with the responses in the door cavity and the vehicle cabin. The following figures would show the same. The acoustic responses for the production door panels are chosen as, naturally, this configuration is the most important. Figure 75 shows the location of the door cavity microphones, the microphone inside the vehicle cabin just next to the door panel as well as the accelerometer location on the door panel used for structural response. This exercise would shed some light on whether the deformation of the exterior door panel is causing the generation of acoustic pressure inside the door cavity. As the door cavity is fairly small and has an irregular shape, it is unlikely that the door cavity would have any modes of itself below $256 \mathrm{~Hz}$. Hence, most likely the acoustic response in the door cavity would be due to the interaction with the vehicle cabin as well as with the exterior door panel. The exterior door panel is basically a sheet metal and has low lateral stiffness making it susceptible to deflection.

Figure 75 shows the response at Driver door due to force inputs at the rear shaker.

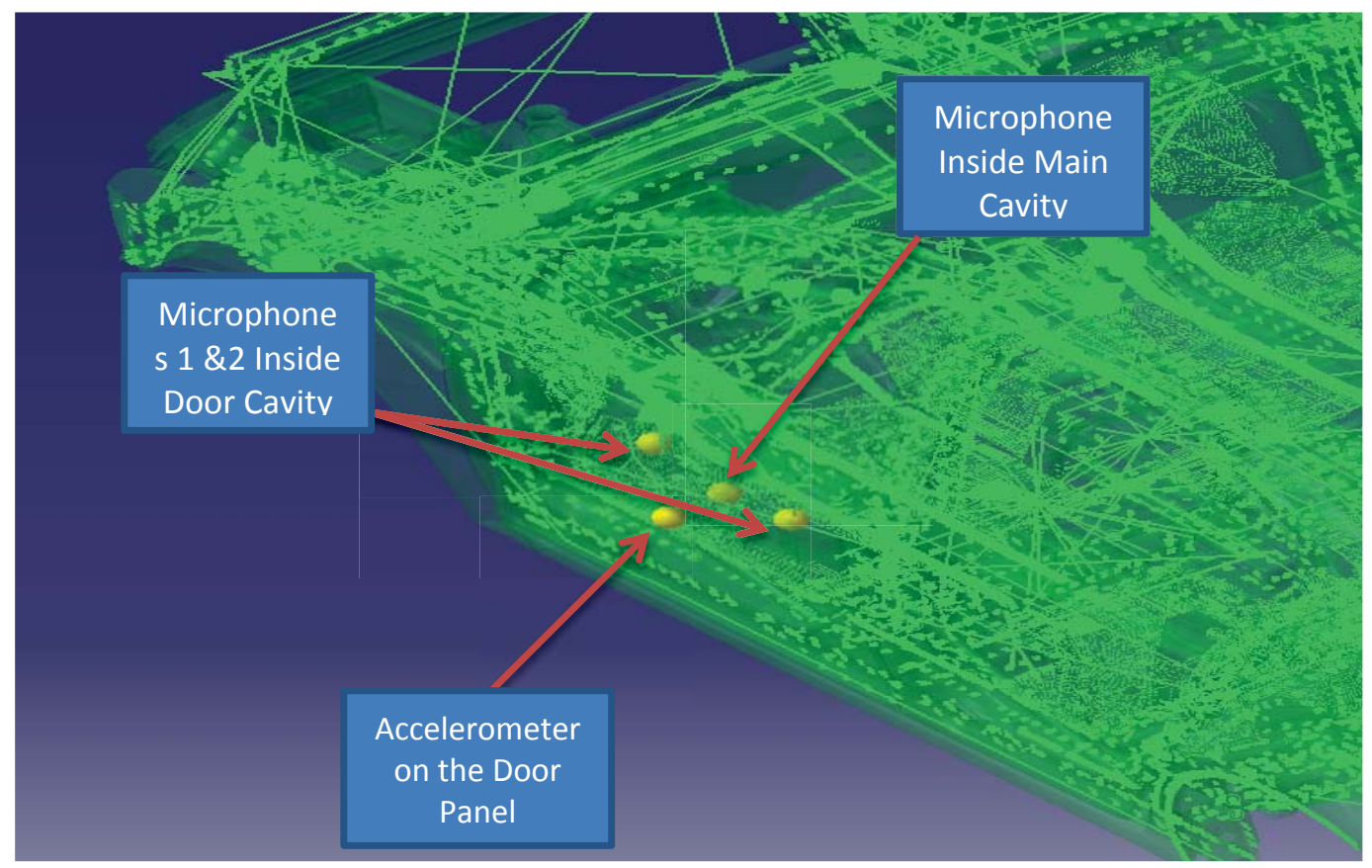

Figure 74: Location of Transducers on the Door, Door Cavity and Vehicle Cabin 


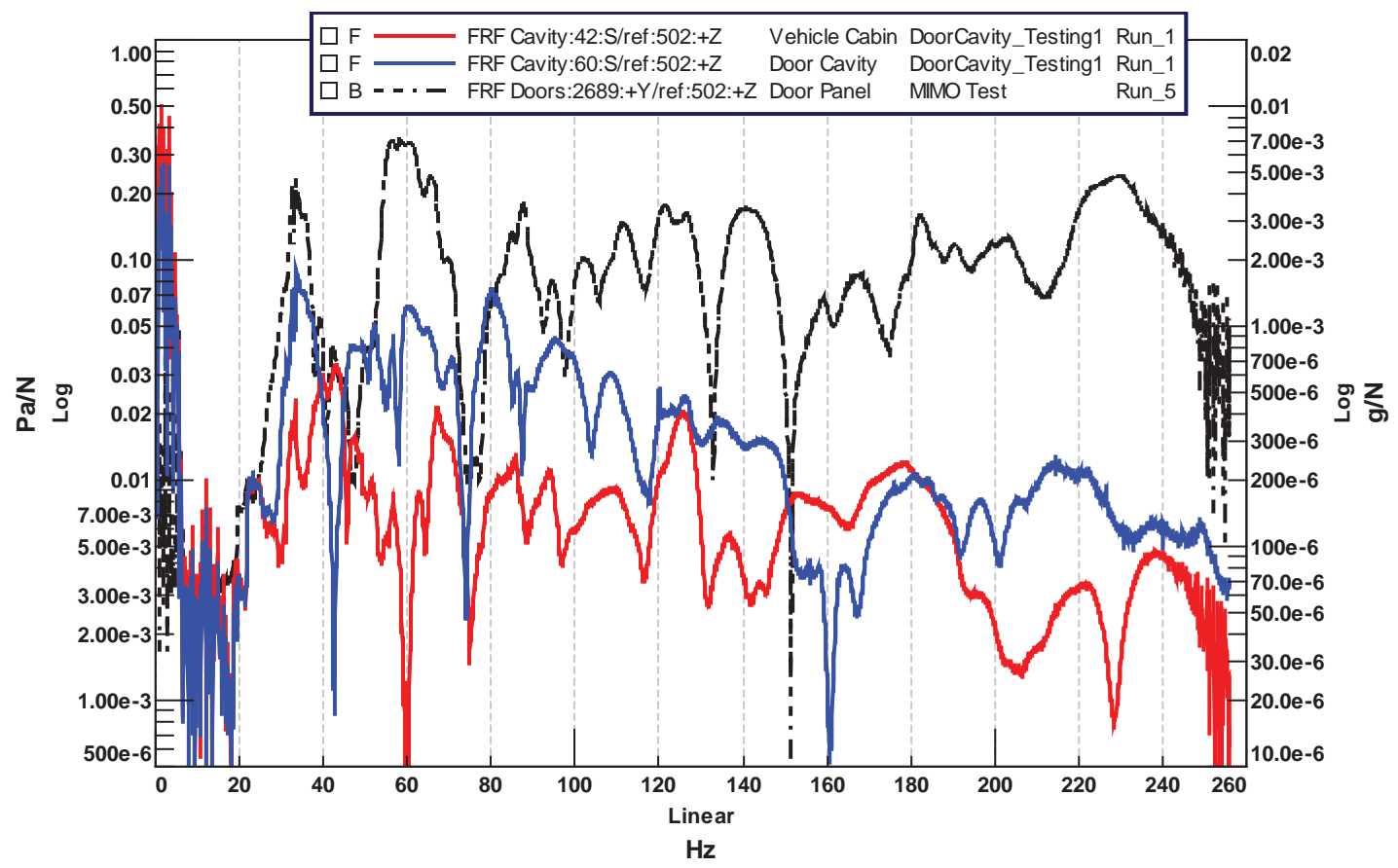

Figure 75: Structural and Acoustic Response for Driver door due to Rear Shaker

The acoustic response in the door cavity is similar to the structural response of the door panel.

The acoustic FRF for the vehicle cabin has peaks which in most cases match up with the door cavity peaks. From the above plot, it seems that the acoustic response in the door cavity is driven by the deformation of the exterior door panel.

The acoustic response in the vehicle cabin just next to the door cavity is similar. Hence a path from the exterior door panel to the door cavity and then to the vehicle cabin is evident. It is clear that the door cavities do play a role in the acoustic response of the vehicle cabin.

Figure 76 shows the acoustic and structural responses for the front passenger. A peak can be seen for the vehicle cabin FRF at $40 \mathrm{~Hz}$. However, the door cavity response of the exterior door panel does not show a similar peak. The response in the $33 \mathrm{~Hz}$ range for all the three locations is similar. A general trend is seen where the curves follow each other. However, they do not follow each other for the entire frequency range. There are outliers for certain frequency ranges. 


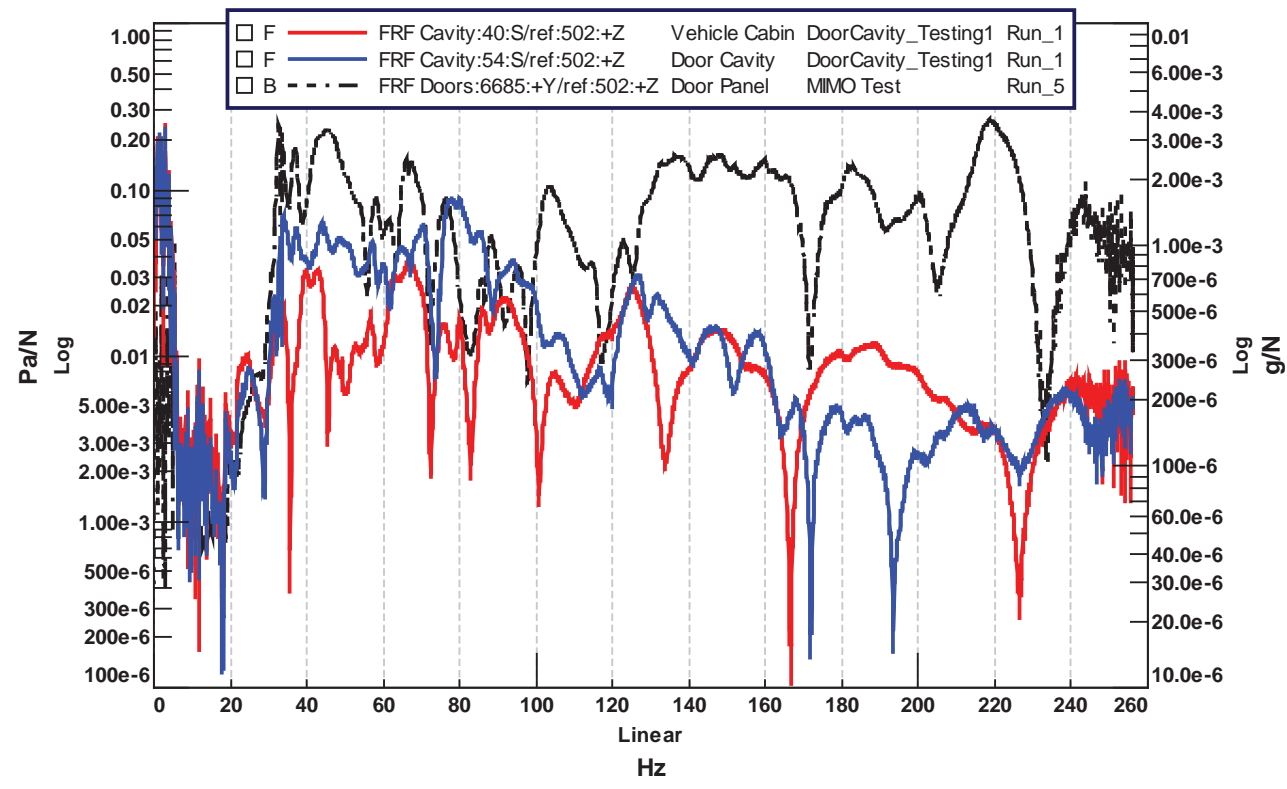

Figure 76: Structural and Acoustic Response for Front Passenger door due to Rear Shaker

Figure 77 and Figure 78 show the responses for the rear passenger behind driver and rear passenger behind front passenger respectively. For these locations as well, a similar trend is seen. The door structure drives the door acoustic response which in turn influences the response in the vehicle cabin.

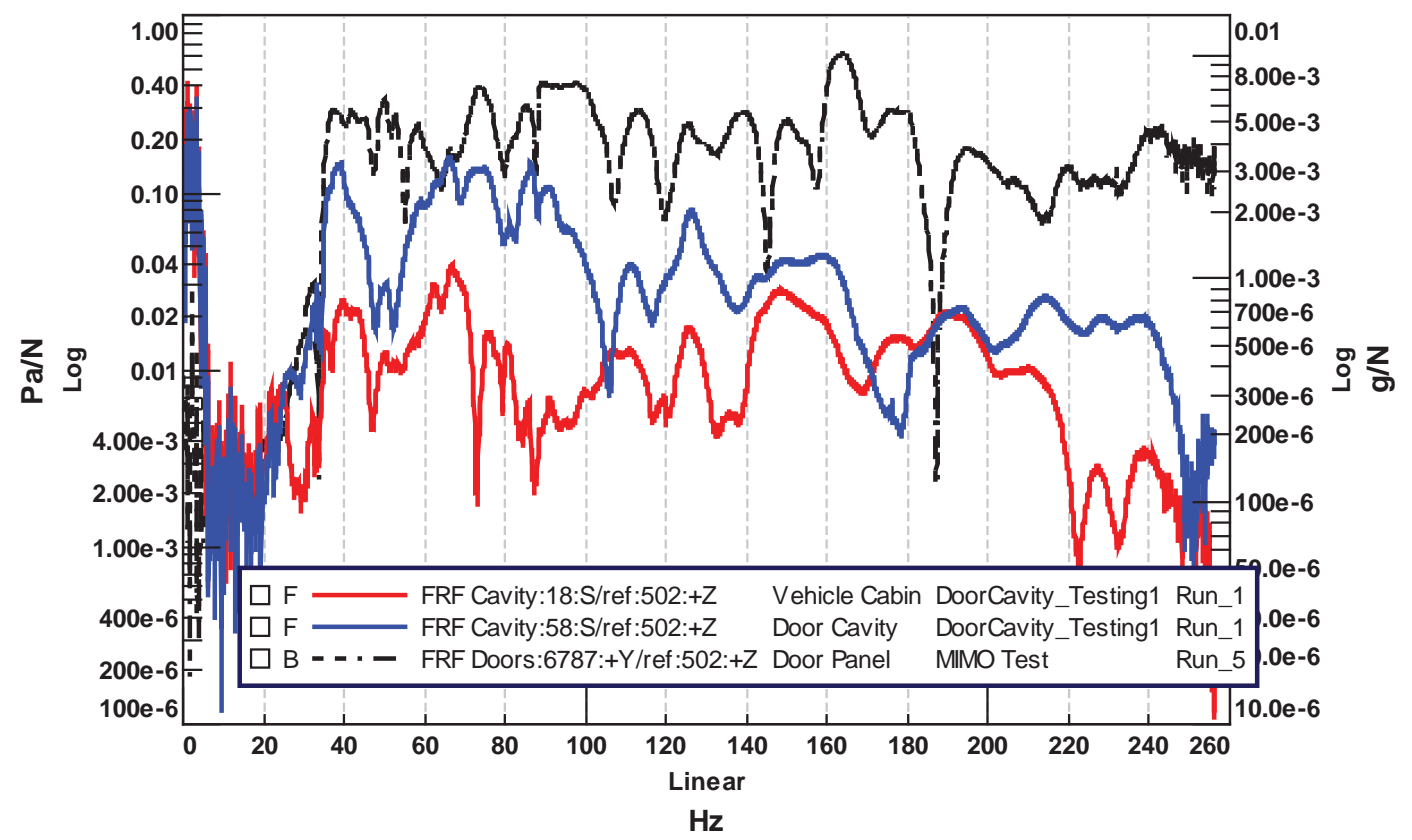

Figure 77: Structural and Acoustic Response for Rear Passenger door behind Driver due to Rear Shaker 


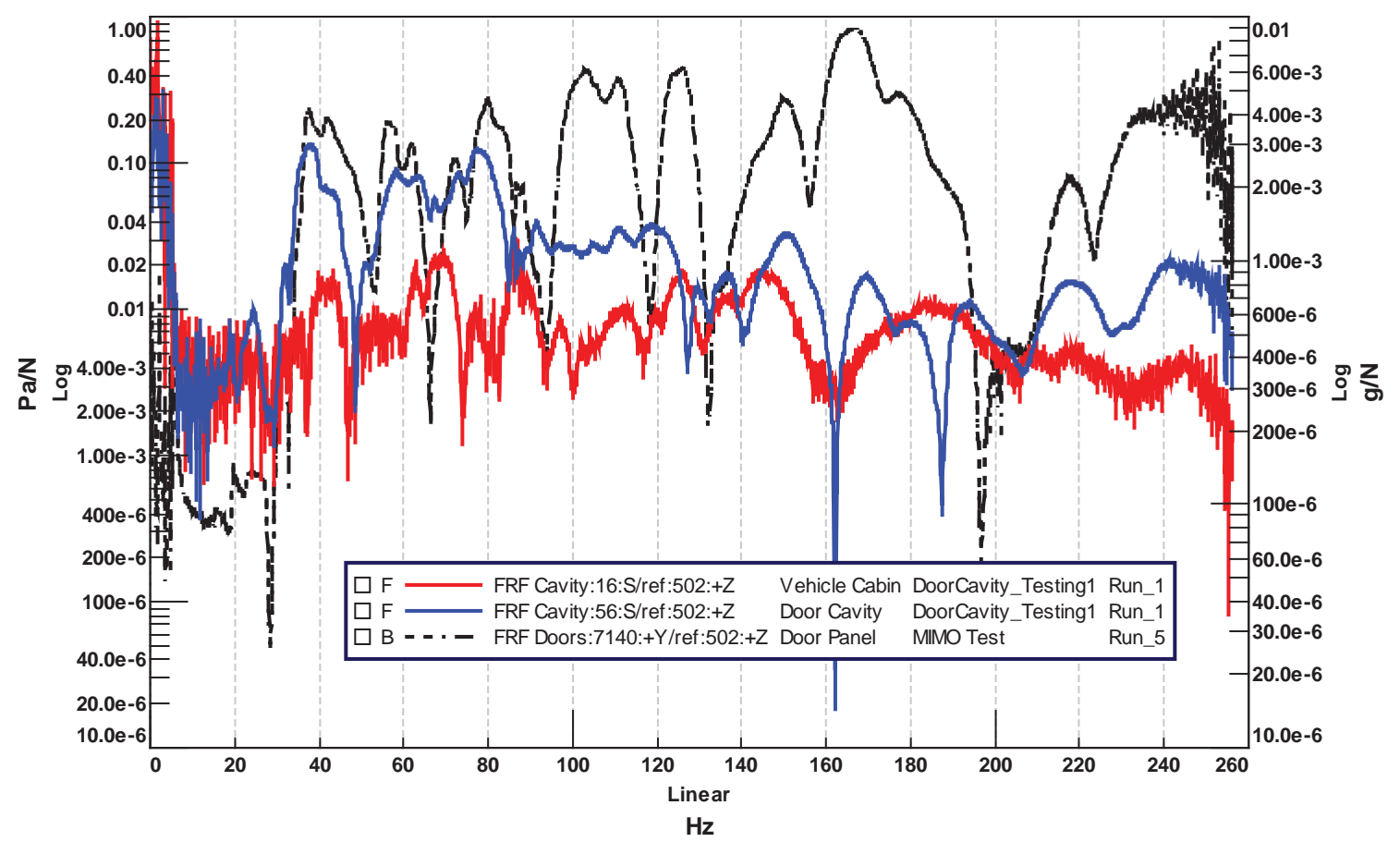

Figure 78: Structural and Acoustic Response for Rear Passenger Door behind Front Passenger due to Rear Shaker

\subsection{Summary of Door Cavity Testing}

An extensive study is performed to understand the behavior and participation of the door cavities to the whole vehicle cavity. If only the DRE is assumed to be the point of interest, then the response due to the rear shaker shows that the door cavities do not play a major role. However, some difference in the amplitude is seen when the input is from the front shaker. The interior production door panels are not completely reflective, but they do absorb a lot of the sound from the door cavities. If the response in the door cavity itself is compared to the response just next to the interior door panel in the vehicle cabin, then a large difference in the amplitudes is observed. The test were also done in conjunction with finite element modeling to understand if there was a need to model the cavities for the acoustic boom phenomenon. From the modeling it was clear that the door cavities do behave like Helmholtz resonators and reduce the acoustic response in the vehicle cabin for certain frequencies. From the testing, it is observed that the door cavities do not affect the acoustic response at the DRE for the boom range. They do affect the responses at other locations. Hence, there is a need to model the door cavities in the finite element model. 


\section{Full Acoustic Modal Test - Structural Input: Seatback Movement Testing}

\subsection{Purpose}

The purpose of this test was to understand the effect of rear seats on the acoustic sensitivity at DRE. This test was done in conjunction with the finite element model in order to understand the correct way of modeling the vehicle cabin and trunk interface [6]. The rear seats of the car behave like a partition between the vehicle cabin and the trunk of the car. The rear seats have foam which helps in acoustic absorption. The seat backs are stiff and can act as barriers. However, some of the air might leak through the cabin and the trunk creating a path for the acoustic waves to travel and interact. This would result in the vehicle trunk influencing the acoustic behavior in the cabin.

\subsection{Description}

In order to understand the effect of the rear seats as well as the interaction between the trunk cavity and the main cavity of the vehicle, the following tests were performed:

a.Testing with rear seats removed

b.Testing with trunk cavity completely blocked with OSB sheets

c. Testing with trunk cavity partially blocked with OSB sheets (path through package shelf speakers)

d.Testing for seatback movement of the OSB sheets

e.Testing for seatback movement of GM production seats

Figure 79 shows the production rear seats removed from the vehicle which makes the vehicle cabin completely open to the trunk. 

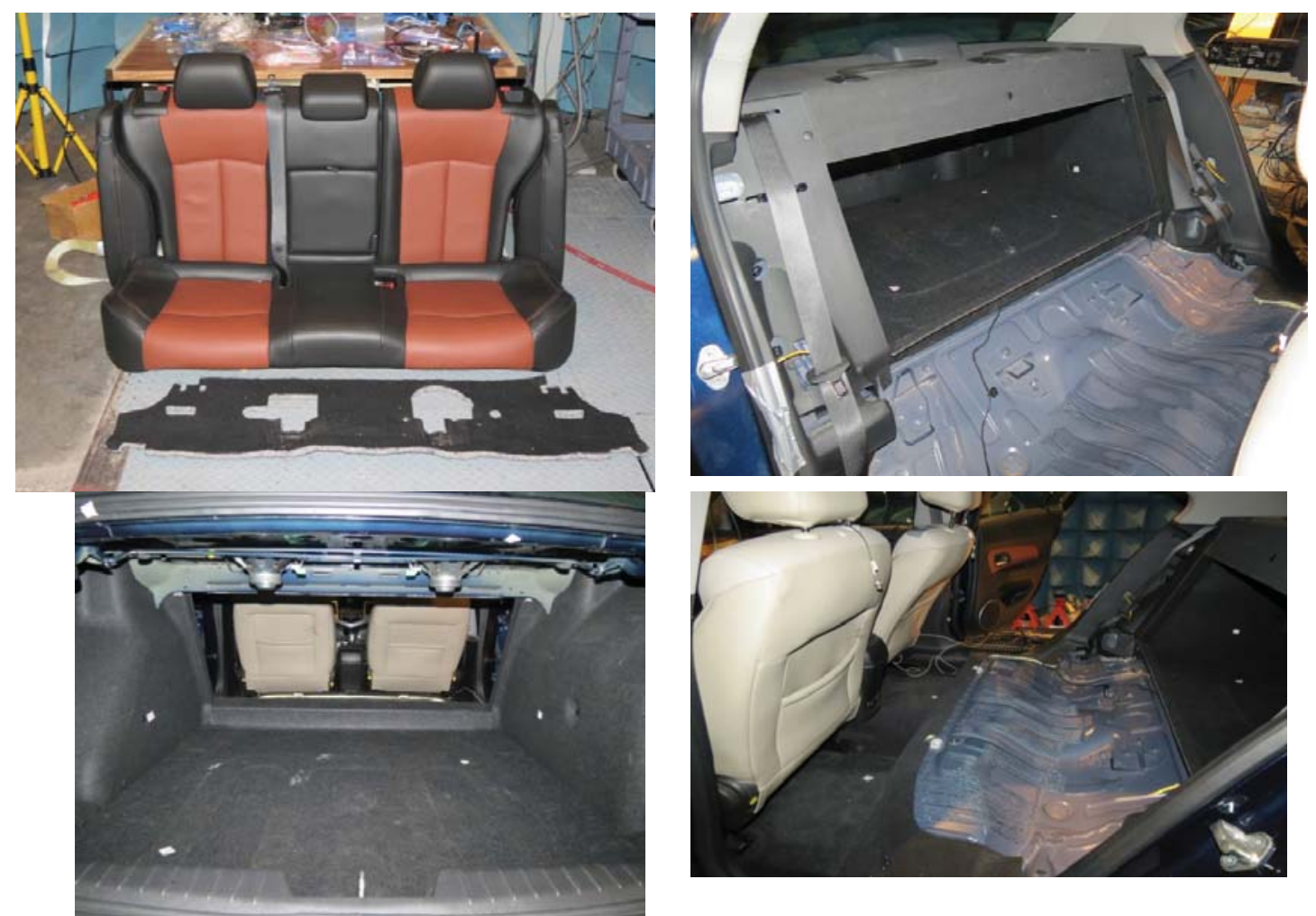

Figure 79: Clockwise from Top: Rear seats removed; Trunk Cavity open to Main Cavity (Perspective View); Trunk Cavity open to Main Cavity (Back View); Trunk Cavity open to Main Cavity (Side View)

Care was taken while removing the rear seats so that no structural member would be damaged. In order to remove the rear seats and perform the testing, the acoustic grid had to be removed and re-installed. Figure 80 shows the setup of the acoustic grid in the vehicle when the rear seats were removed. All efforts were made to ensure that the microphone locations in the acoustic grid were exactly in the same position as they were before removing the grid to remove the rear seats from the vehicle. Figure 81 shows the OSB sheet blocking the trunk cavity from the main cavity. An OSB sheet of the size of the opening was cut to block most of the opening to the trunk. However, due to the contour in the rear seat area, some openings were still present. Smaller OSB sheets were cut and applied from the trunk side of the vehicle. Different acoustic materials such as fiberglass and lead backed foam were also used to seal smaller openings. Finally, a layer of vinyl foam was also applied to seal off minute openings. 


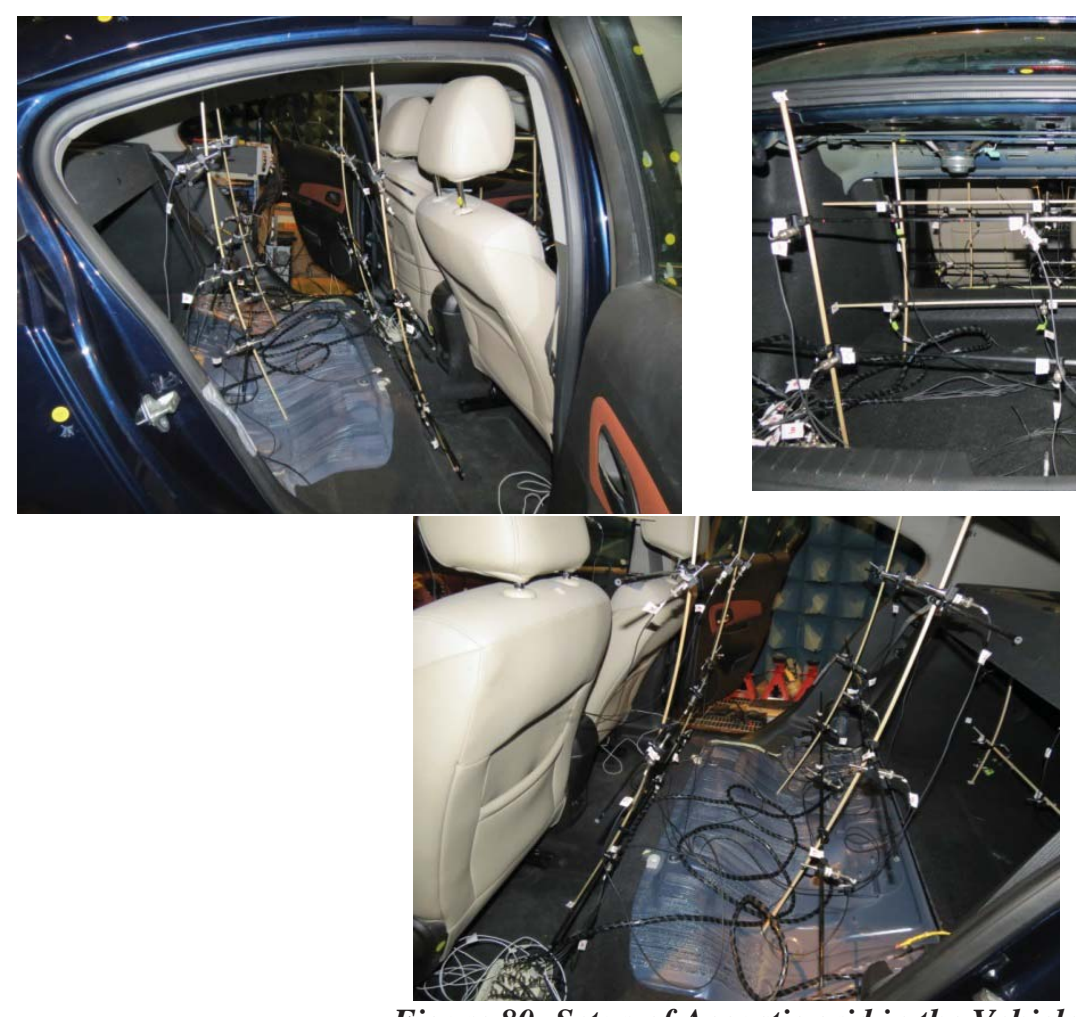

Figure 80: Setup of Acoustic grid in the Vehicle
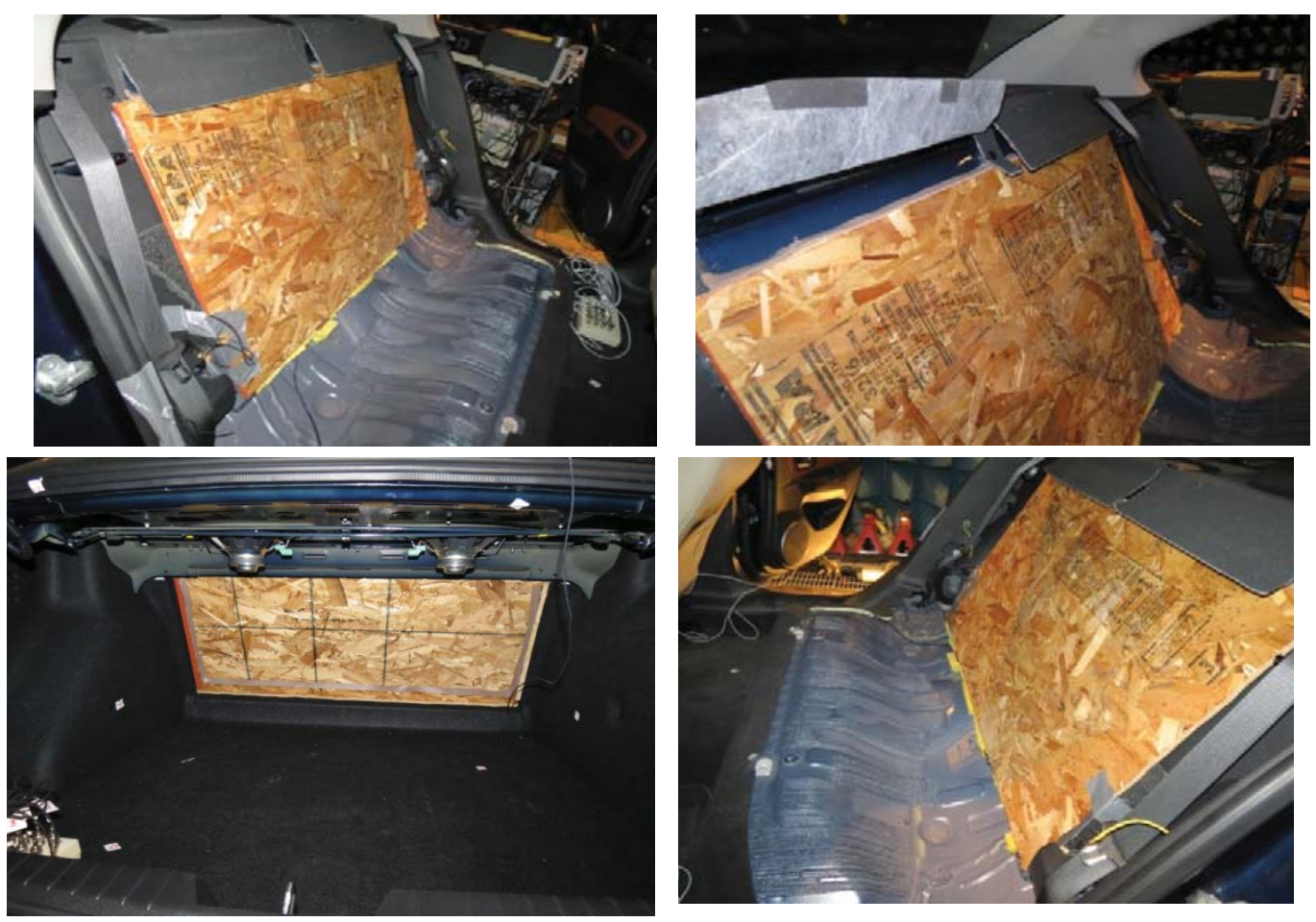

Figure 81: OSB sheet blocking Main Cavity from the Trunk Cavity 
Figure 82 shows how the package shelf speakers were covered by the OSB sheets to completely block the main cavity from the trunk cavity.

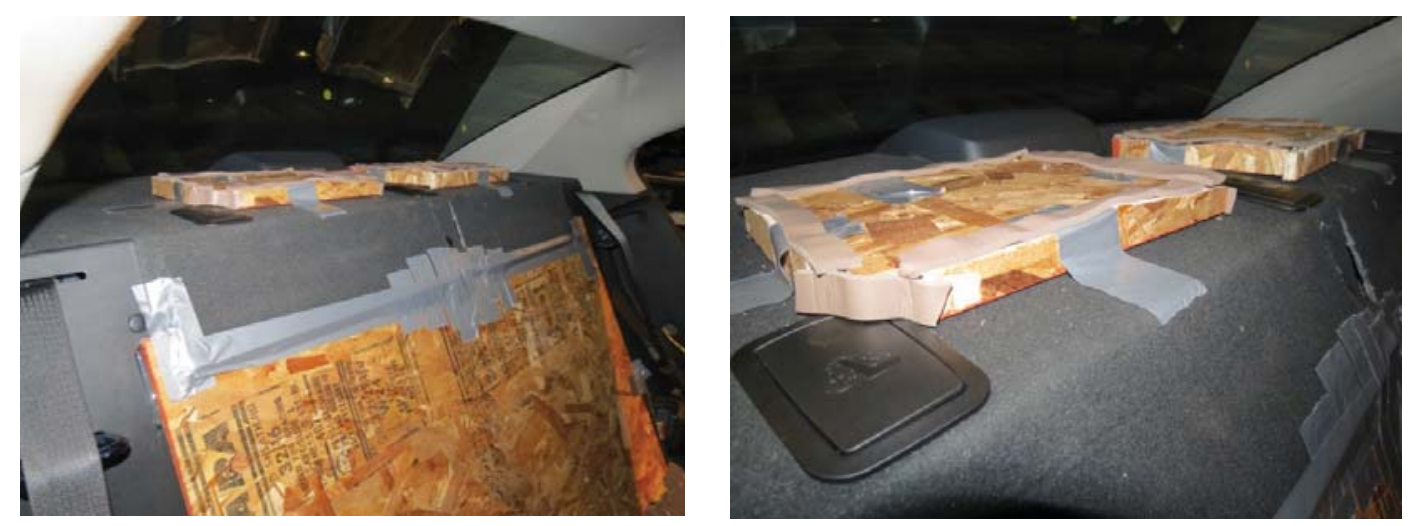

Figure 82: Package shelf speakers blocked by OSB sheets

Figure 83 shows the acoustic sensitivity at DRE for the following configurations: Baseline (All production parts in the vehicle), Rear Seats removed, Trunk cavity blocked by OSB sheets and Trunk cavity acoustically connected to the main cavity by keeping the package shelf speakers untouched. The frequency range of $30-75 \mathrm{~Hz}$ is shown in Figure 83.

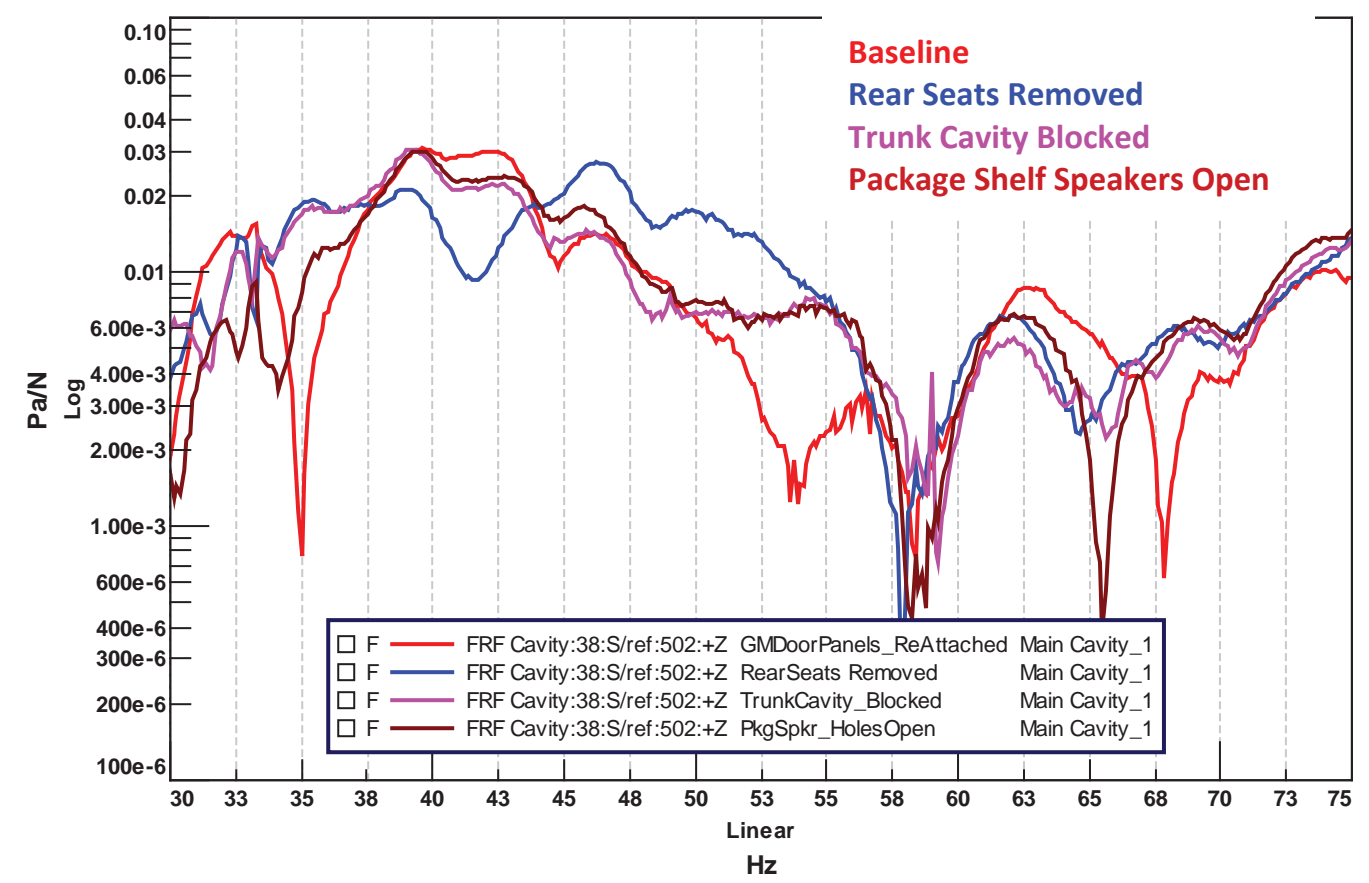

Figure 83: Acoustic Sensitivity at DRE due to Rear Shaker 
From the above figure, it can be seen that in the boom range of $40 \mathrm{~Hz}$, the sensitivity is low when the trunk cavity is completely open to the main cavity. The sensitivity is more or less similar for the other configurations. The sensitivity remains the same whether the package shelf speakers are kept open or closed.

Figure 84 shows the acoustic sensitivity at DRE due to the front shaker. In this case the sensitivity for the baseline condition is highest while, the sensitivity for the other three configurations is lower. All the curves show similar sensitivity above $48 \mathrm{~Hz}$.

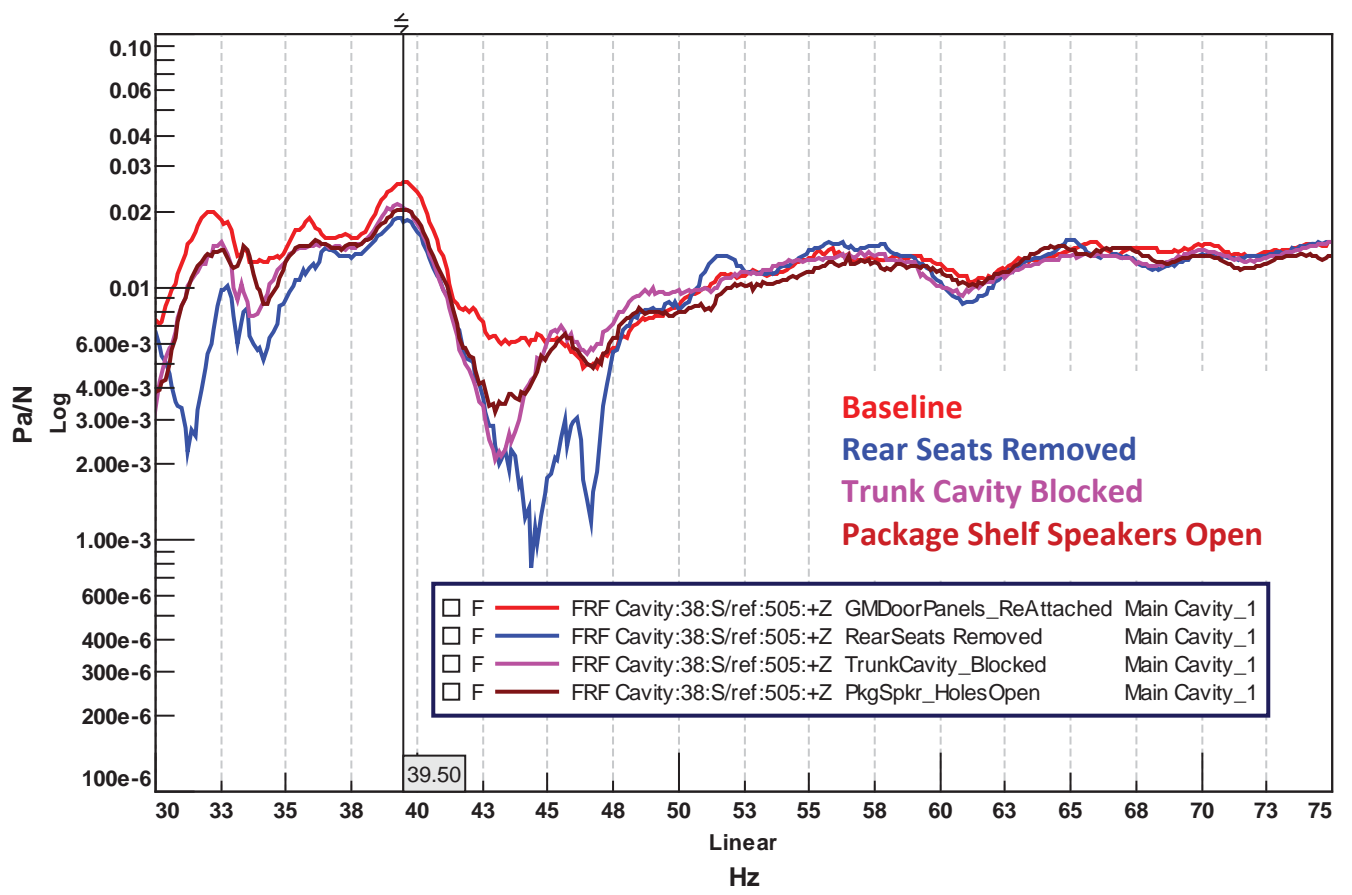

Figure 84: Acoustic Sensitivity at DRE due to Front Shaker

Figure 85 shows the acoustic mode in the boom range. With the rear seats removed, the trunk cavity and main cavity act as one big cavity. The mode shows greater fluctuation of acoustic pressure in the trunk and rear seats region than in the front seats. This mode is a little similar to the rigid body acoustic mode where the whole cavity shows uniform and in-phase pressure fluctuation.

Figure 86 shows the acoustic mode in the boom range when the trunk cavity was completely blocked with the help of OSB sheets. This mode shows slight fluctuation in acoustic pressure in the front seats region, whereas the major fluctuation in pressure occurs in the trunk. The pressure transition seems to be from the trunk followed by the rear seats and then to the front seats. This may show that even though the OSB sheets were used to completely seal of the trunk cavity, small gaps still existed due to which the acoustic pressure in the trunk could influence the main cavity. 


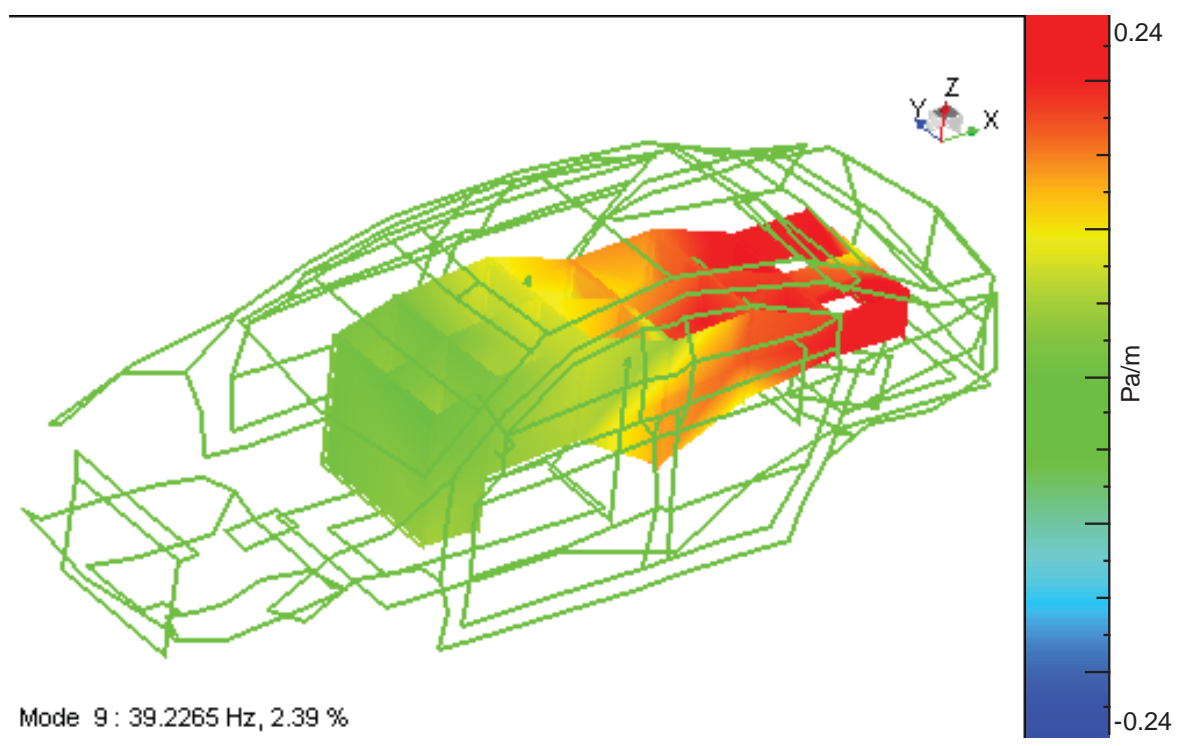

Figure 85: Acoustic Mode at 39 .2 Hz-Rear Seats Removed

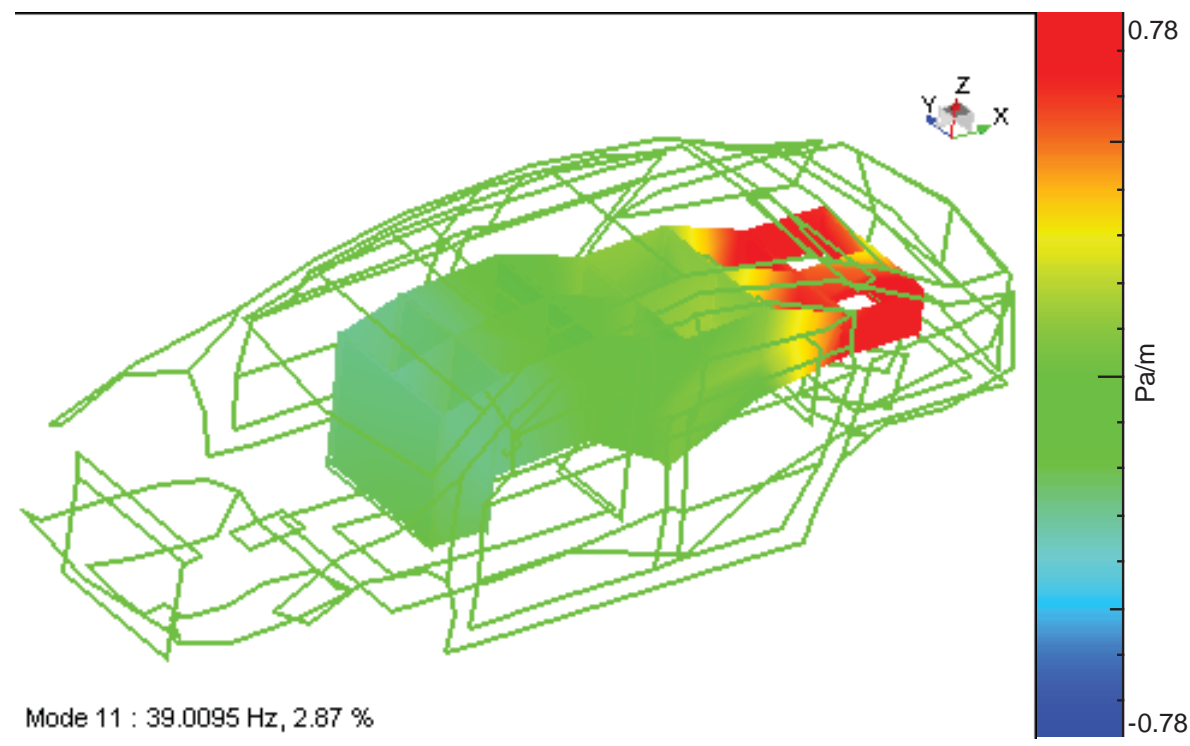

Figure 86: Acoustic Mode at 39 Hz-Trunk Cavity Blocked

Figure 87 shows the acoustic mode at $40 \mathrm{~Hz}$ when the trunk cavity is completely blocked. In this mode as well, the major acoustic pressure fluctuations take place in the trunk cavity. 


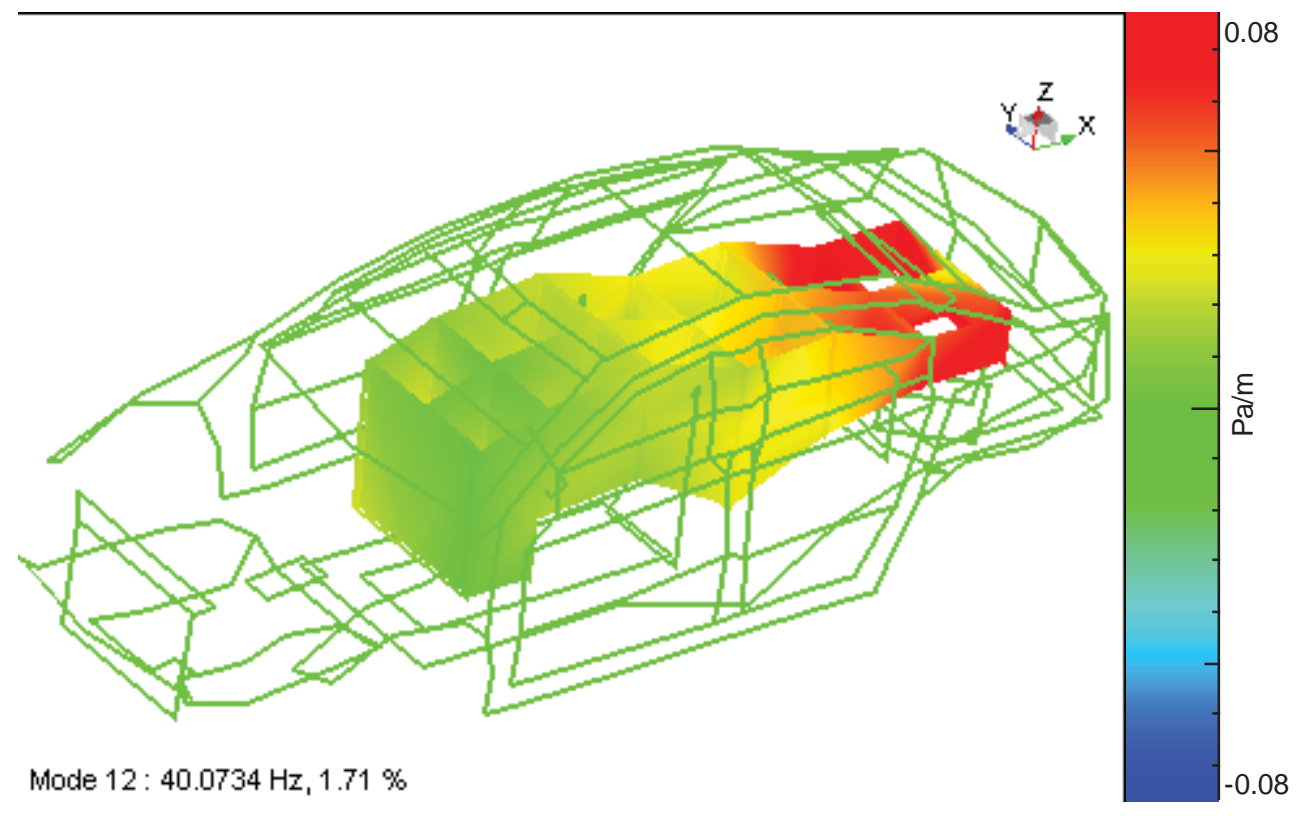

Figure 87: Acoustic Mode at Trunk Cavity Blocked

Figure 88 shows the acoustic mode at $43 \mathrm{~Hz}$ when the trunk cavity is completely blocked from the main cavity. In this mode, a first acoustic mode is seen where the vehicle cabin and the trunk cavity are out of phase with each other. As seen in earlier cases, the pressure fluctuation in the trunk cavity is larger than in the main cavity.

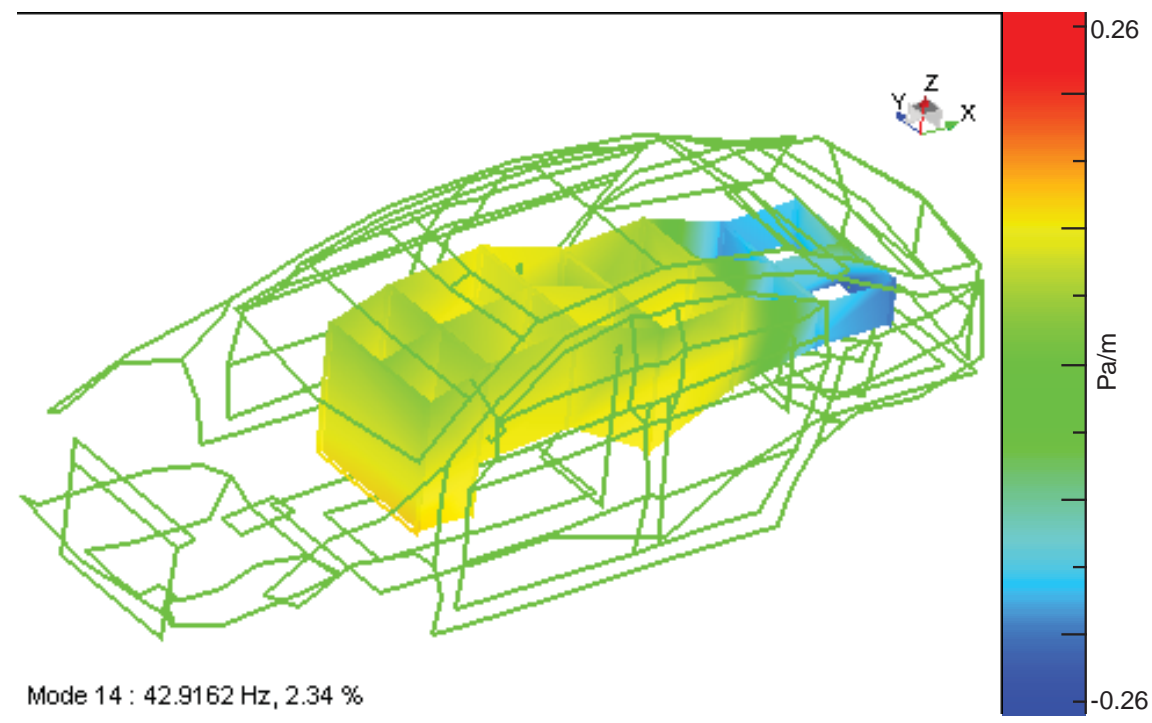

Figure 88: Acoustic Mode at 42.9 Hz-Trunk Cavity Blocked

Figure 89 shows the acoustic mode at $39.7 \mathrm{~Hz}$ when the trunk cavity is acoustically connected to the main cavity through the package shelf speakers. A mode similar to a rigid body acoustic mode is seen. The acoustic pressure in the trunk cavity and main 
cavity is similar and in phase with each other. This is interesting to know as a similar mode was not seen when the rear seats were removed and the main cavity and trunk cavity were completely connected.

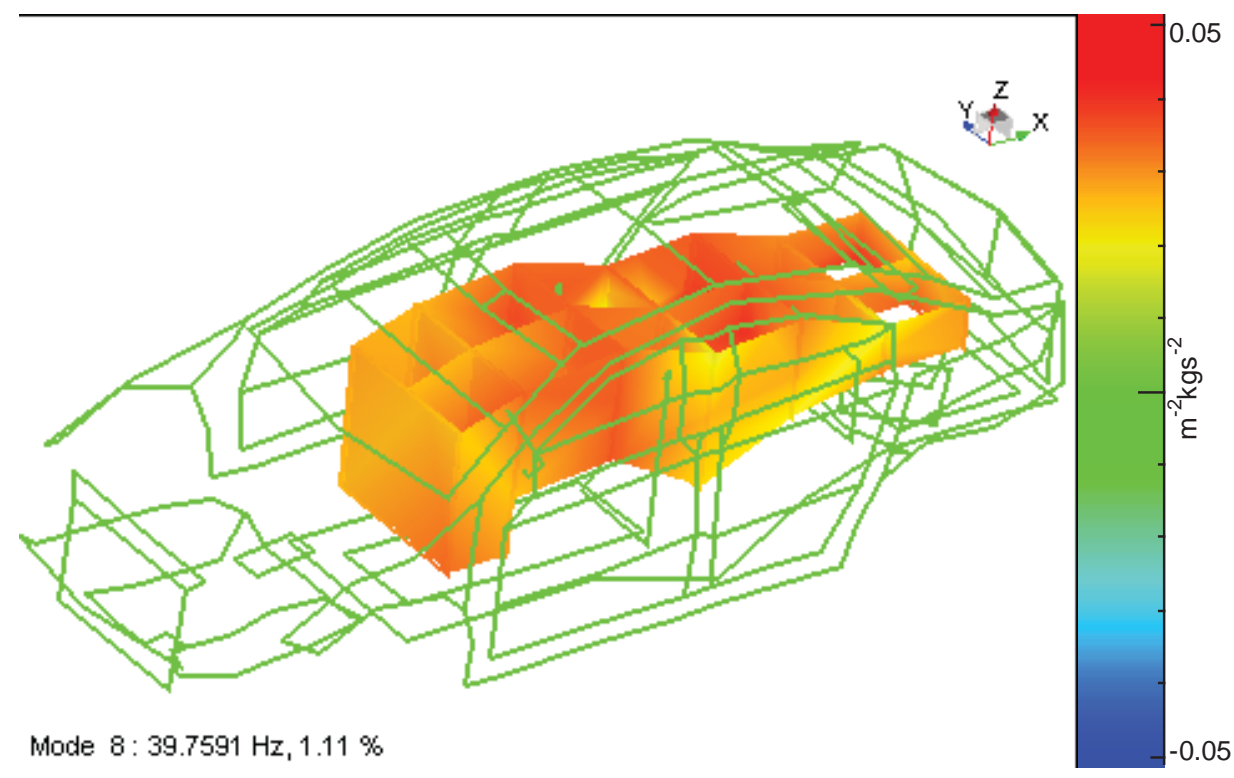

Figure 89: Acoustic Mode at $39.76 \mathrm{~Hz}$-Speakers Open

Figure 90 shows the acoustic mode at $43 \mathrm{~Hz}$ for the package speakers open configuration. This mode is similar to the one seen in Figure 88. The acoustic pressures in the main cavity and trunk cavity are out of phase with each other.

Figure 91 shows the setup to measure the structural response of the OSB sheet due to the shaker inputs. The seatback movement test would help to understand the motion of the seat and its interaction with the acoustic pressure in the trunk cavity as well as the main cavity. A total of 8 tri-axial accelerometers were mounted on the OSB sheet as well as on the production seats to measure the movement. 


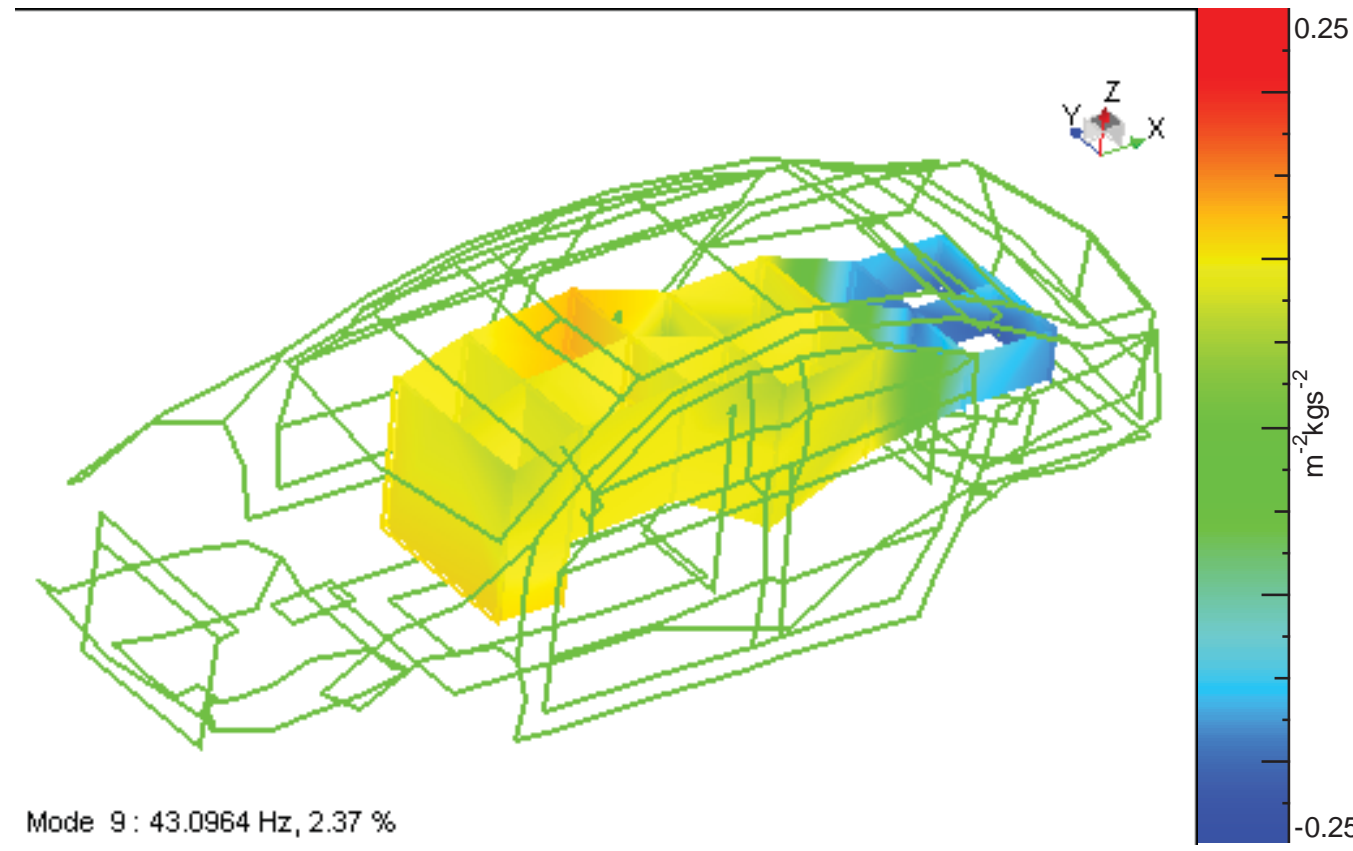

Figure 90: Acoustic Mode at 43 Hz-Package Speakers Open

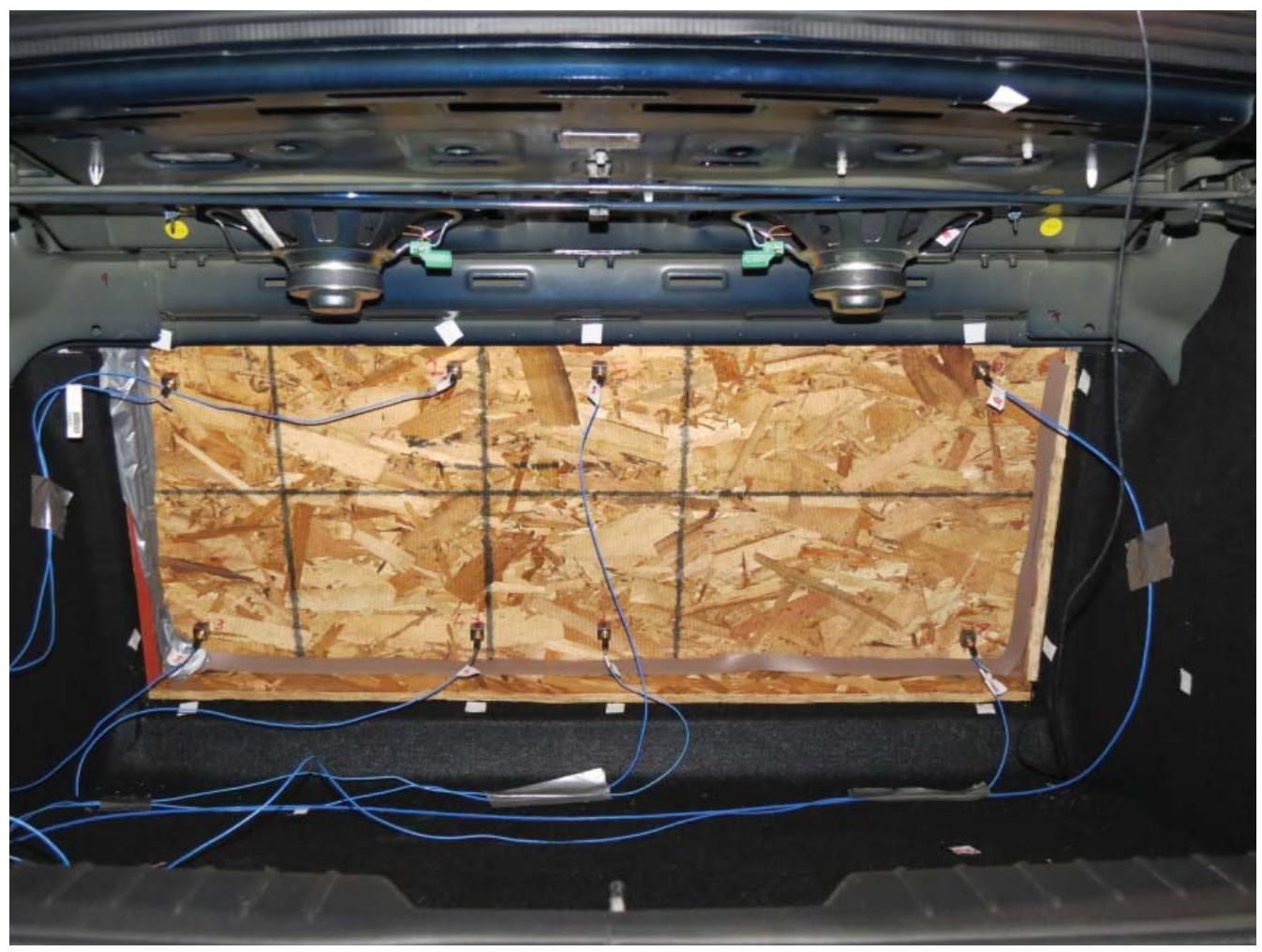

Figure 91: Transducers on OSB sheet for Seatback movement Testing 
Figure 92 shows the tri-axial accelerometers mounted on GM production seats so as to measure the seat back movement. It was ensured that the tri-axial accelerometers were mounted in similar positions as the OSB sheets. The rear seats have a $60-40$ split for easier loading and unloading of cargo in the vehicle. Each section had four transducers to record the movement.

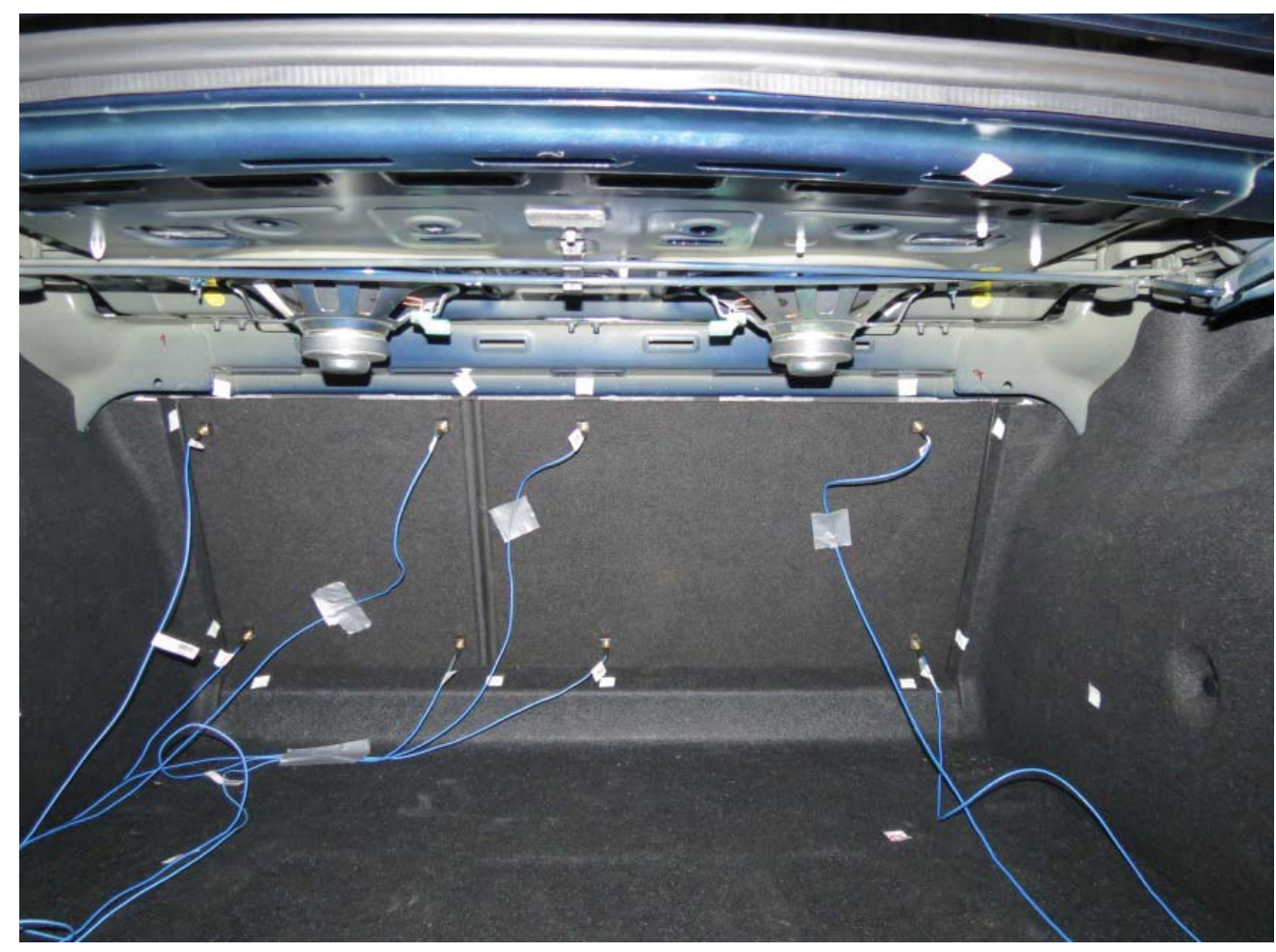

Figure 92: Transducers on GM production seats for Seatback Movement Testing

The Seatback Movement Testing was performed in four configurations as under:

- Testing with OSB sheets with package shelf speakers closed

- Testing with OSB sheets with package shelf speakers open

- Testing with GM Seats with package shelf speakers closed

- Testing with GM Seats with package shelf speakers open

Figure 93 shows the mode shape at $39 \mathrm{~Hz}$ for the configuration of OSB sheets with the package shelf speakers closed. In this case, theoretically, the trunk cavity and the main cavity are completely separated from each other. For visualization purposes some of the parts are not shown as they do not indicate any deformation. The seatback geometry can be seen in between the cavity towards the trunk. As the seat moves forward, high pressure is created in the main cavity and as it moves backwards, a high pressure is created in the trunk. The seatback has a complex motion. The OSB sheet shows some flexing and is not moving like a rigid structure. 


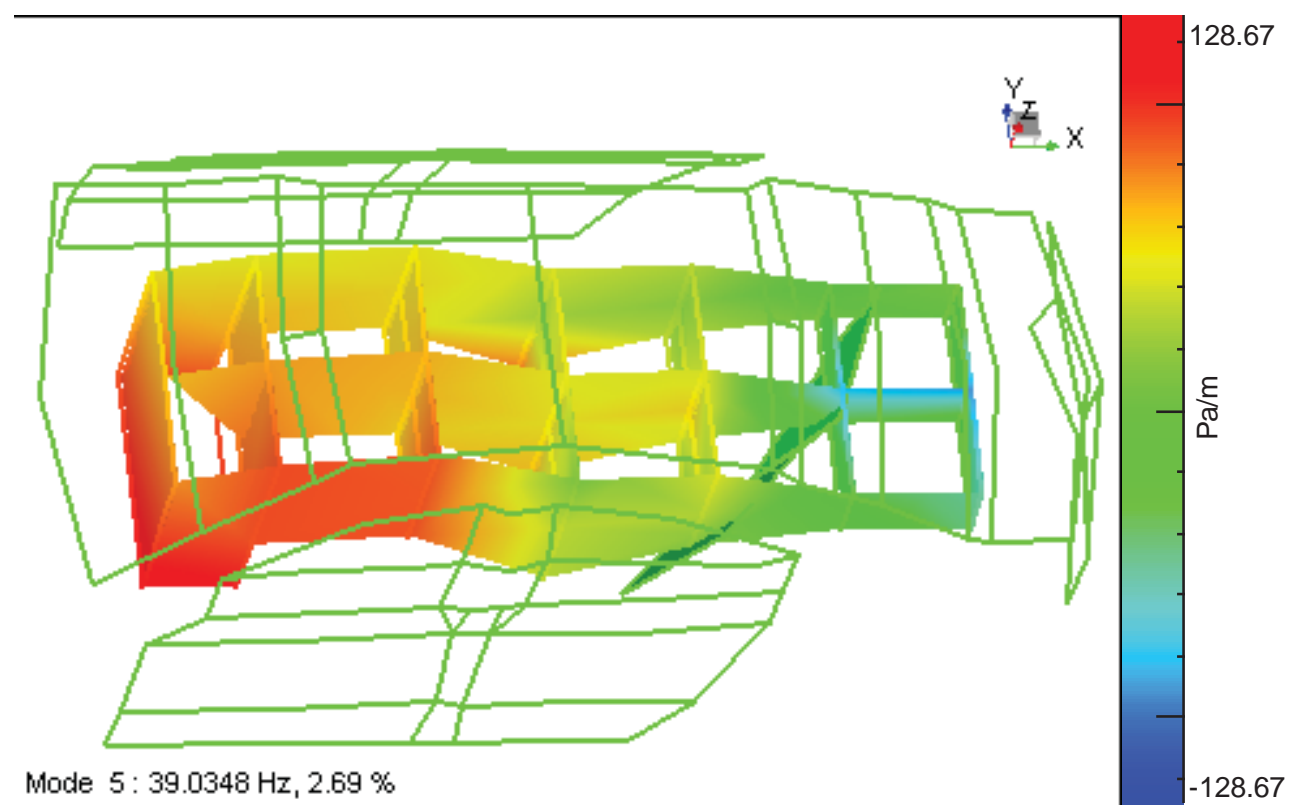

Figure 93: Vibro-Acoustic Mode at $39 \mathrm{~Hz}$ (OSB-Speaker Closed)

Figure 94 shows the vibro-acoustic mode at $39.2 \mathrm{~Hz}$ for the same configuration. In this case, the movement of the seatback is similar to the previous mode shape as seen in Figure 92; however the acoustic pressure developed in the cavity is higher.

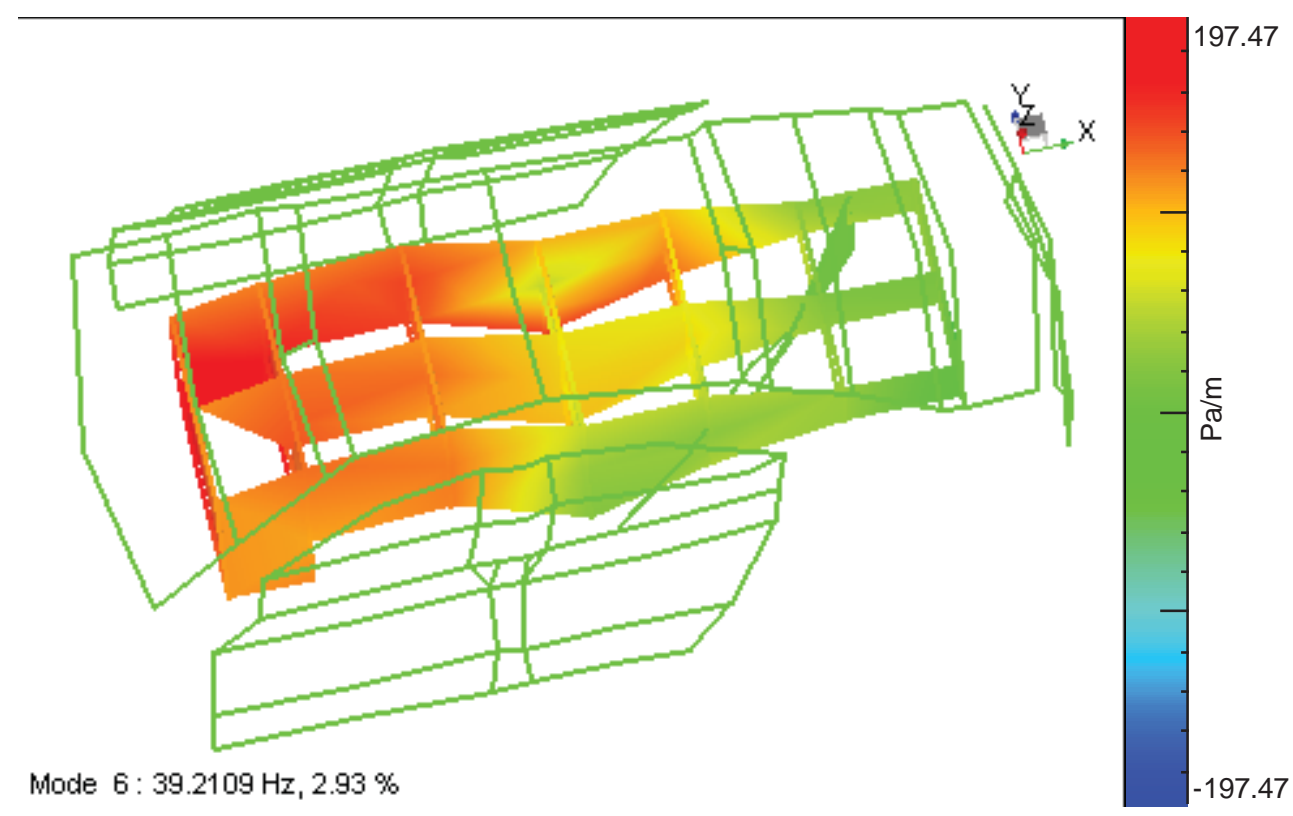

Figure 94: Vibro-Acoustic Mode at 39.2 Hz(OSB-Speaker Closed) 
Figure 95 shows the $42.2 \mathrm{~Hz}$ vibro-acoustic mode. The OSB sheet moves as a single entity in this mode. There is only a small deflection for the OSB sheet. Fluctuation in acoustic pressure is mainly in the trunk cavity while the main cavity has minor fluctuations.

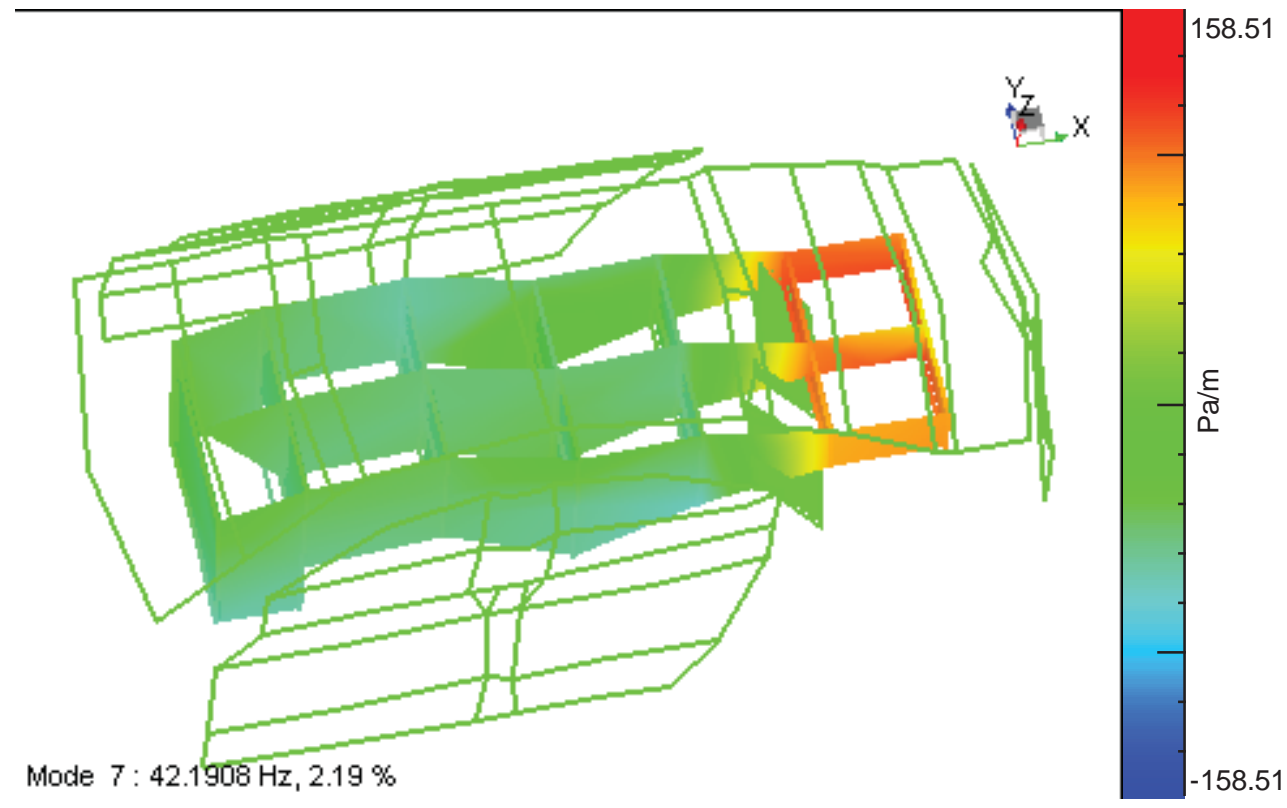

Figure 95: Vibro-Acoustic Mode at 42.2 Hz(OSB-Speaker Closed)

Figure 96 shows the $39 \mathrm{~Hz}$ mode when the package shelf speakers are untouched. This makes the trunk cavity acoustically connected to the main cavity.

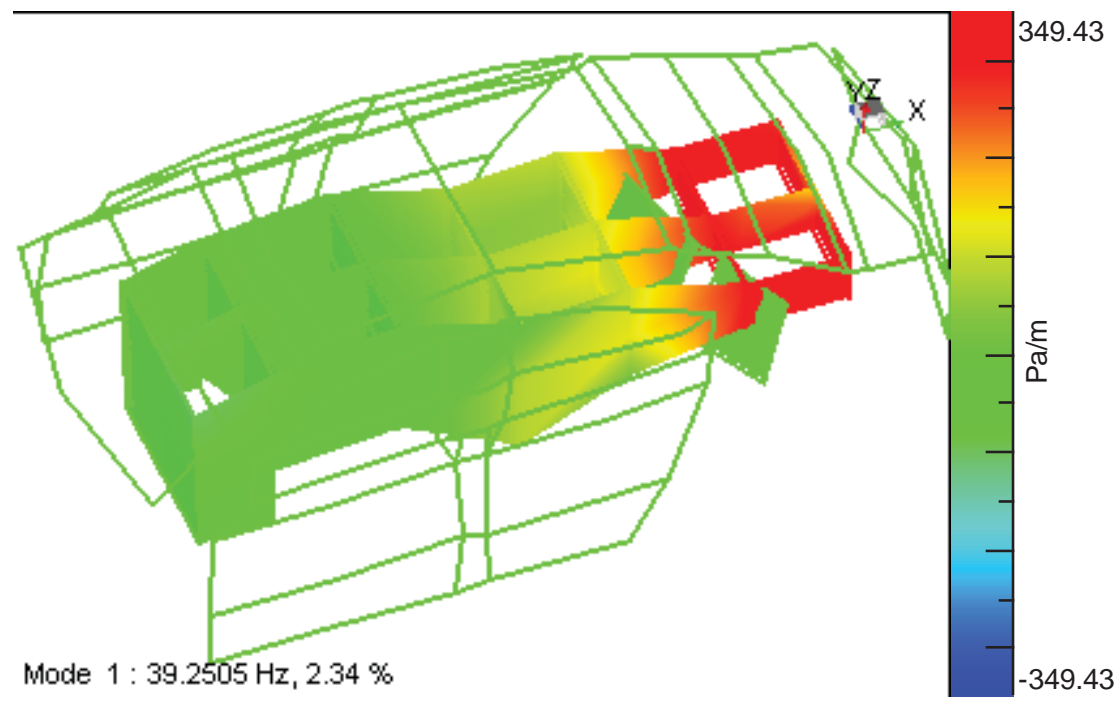

Figure 96: Vibro-Acoustic Mode at $39 \mathrm{~Hz}$ (OSB-Speaker Open) 
This mode is mainly the trunk mode with minor fluctuations in acoustic pressure in the main cavity. In this mode as well, it seems that the seatback is driving the fluctuations in acoustic pressure in the trunk cavity.

Vibro-acoustic mode at $39.3 \mathrm{~Hz}$ is shown in Figure 97. The OSB sheet is flapping about its vertical central axis. This is a complex acoustic mode where high pressure is partially developed in the trunk and the main cavity.

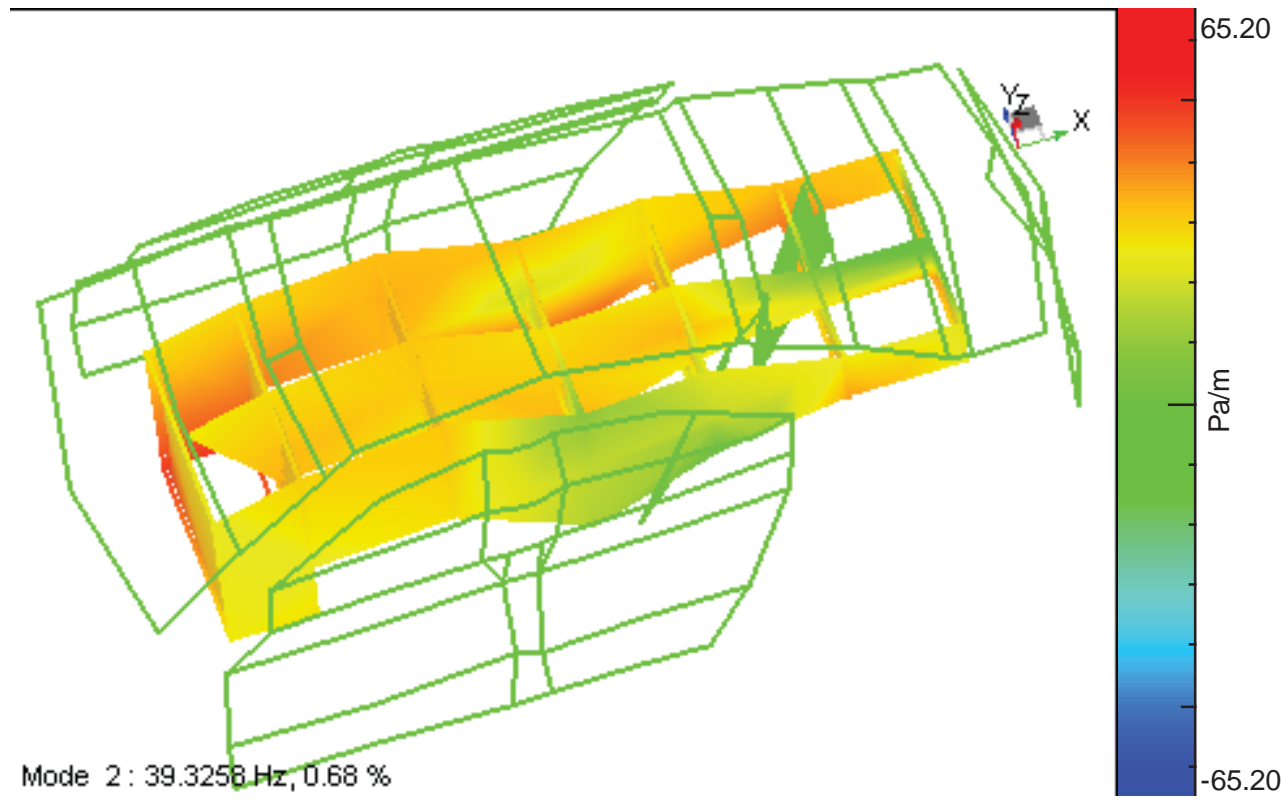

Figure 97: Vibro-Acoustic Mode at $39.3 \mathrm{~Hz}$ (OSB-Speaker Open)

Vibro-acoustic mode at $42.3 \mathrm{~Hz}$ is shown in Figure 98. This is again a trunk mode. There is negligible pressure fluctuation in the main cavity.

Figure 99 shows the mode when the GM production seats are re-installed in the vehicle and the speakers are covered with the OSB sheets. The mode occurs at $39.2 \mathrm{~Hz}$. The GM seats have 60/40 split for easier loading and unloading of the cargo. However, this shape results in the independent motion of the seats. There is phase lag between the two parts and a flip-flop motion of the seats is observed. As seen in the earlier modes, the pressure fluctuation in the trunk is higher than that of the main cavity. 


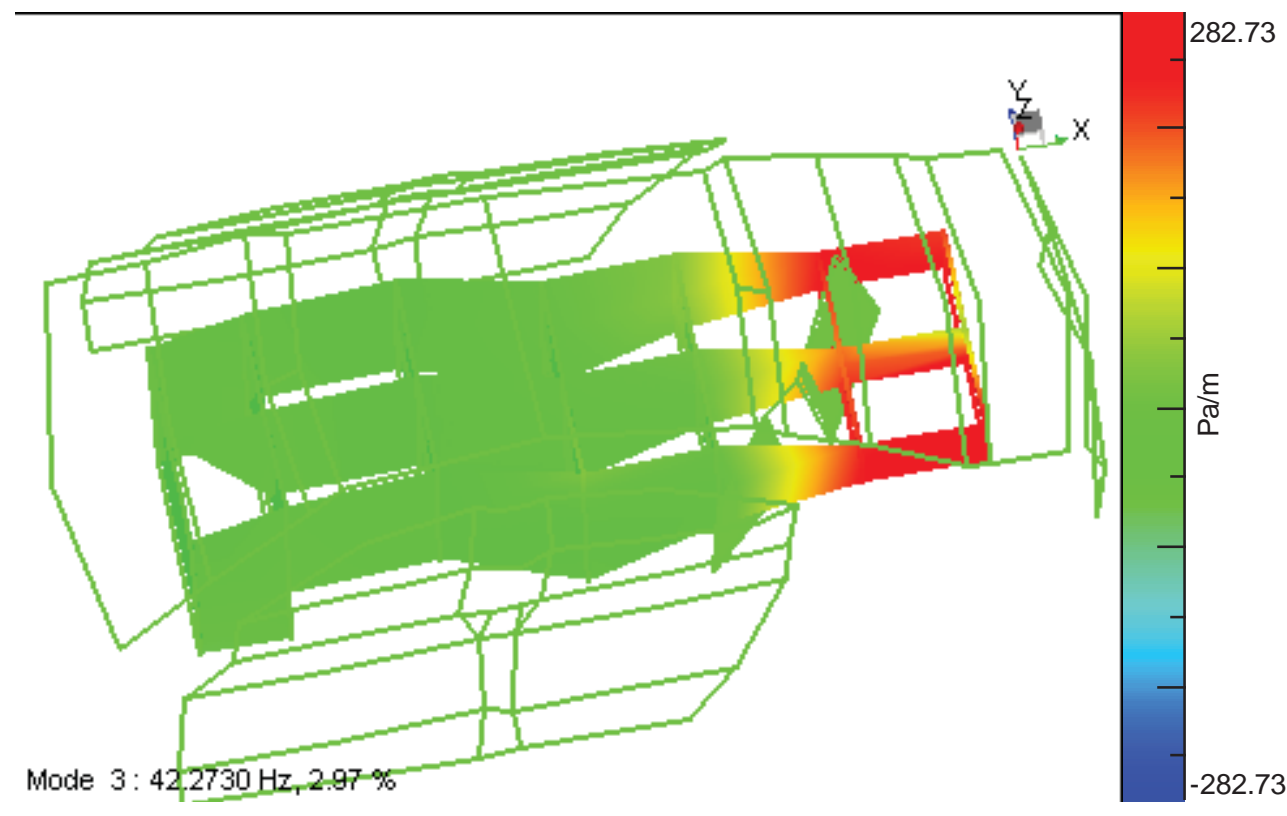

Figure 98: Vibro-Acoustic Mode at $42.3 \mathrm{~Hz}$ (OSB-Speaker Open)

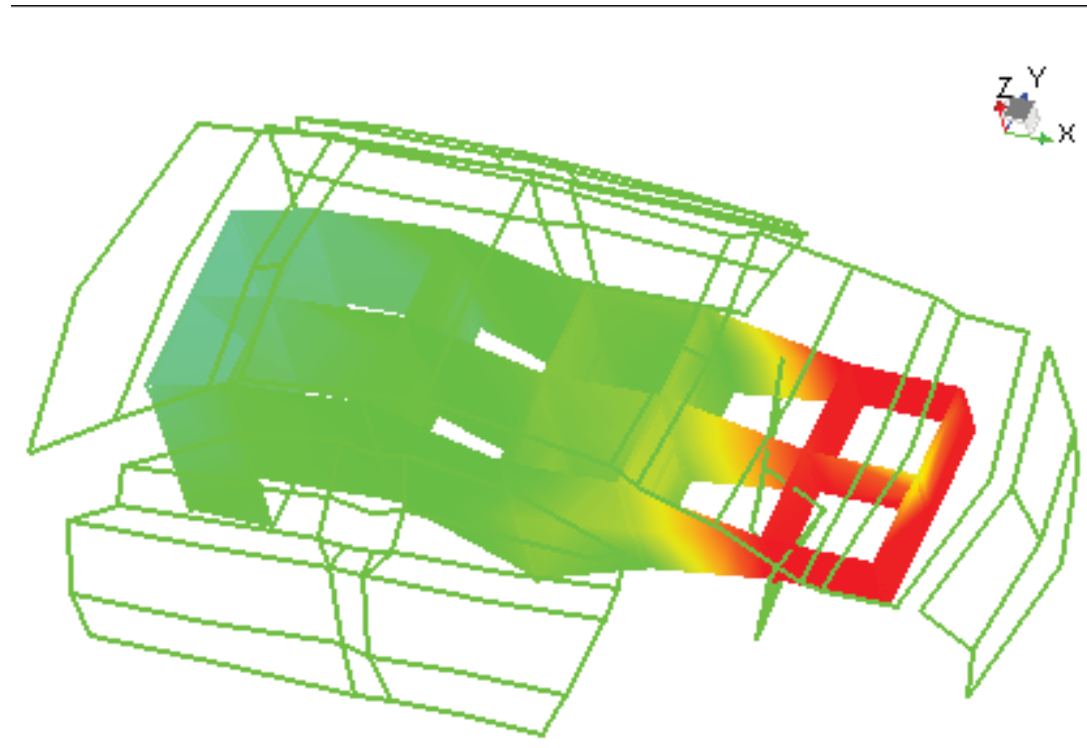

Mode $13: 39.2186 \mathrm{~Hz}, 3.31 \%$

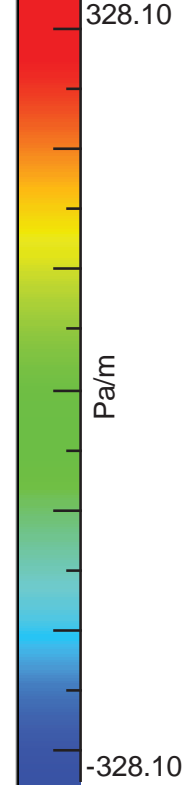

Figure 99: Vibro-Acoustic Mode at 39.2 Hz(GM Seats Speaker Closed)

Figure 100 shows the mode shape at $40.5 \mathrm{~Hz}$. There is theoretically, no acoustic connection between the main cavity and the trunk cavity. 
In this mode shape, it is seen that the acoustic pressure is driving the seat back. High pressure developed in the trunk cavity, makes the seat move forward. It moves backward when a low pressure is generated in the trunk.

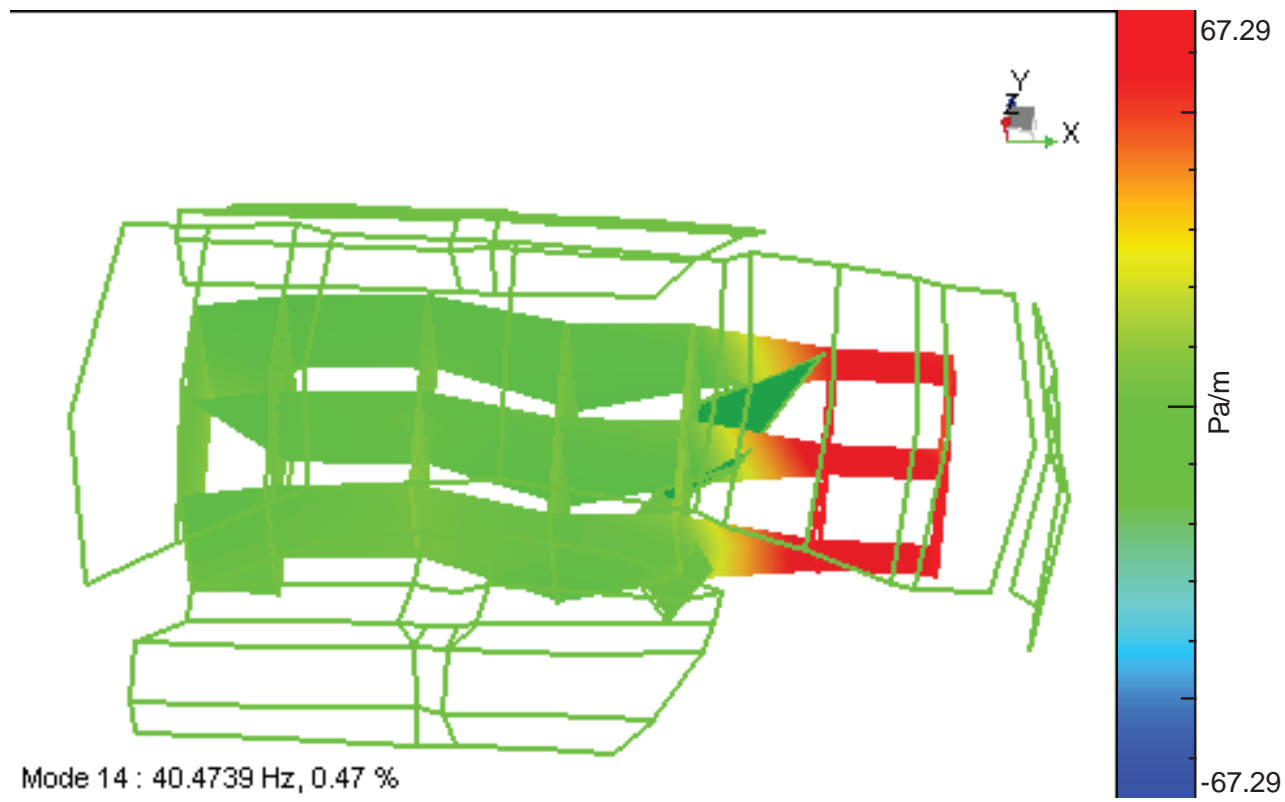

Figure 100: Vibro-Acoustic Mode at $40.5 \mathrm{~Hz}$ (GM Seats Speaker Closed)

Vibro-acoustic mode shape at $42.5 \mathrm{~Hz}$ for the configuration of GM Seats with speakers closed is shown in Figure 101.

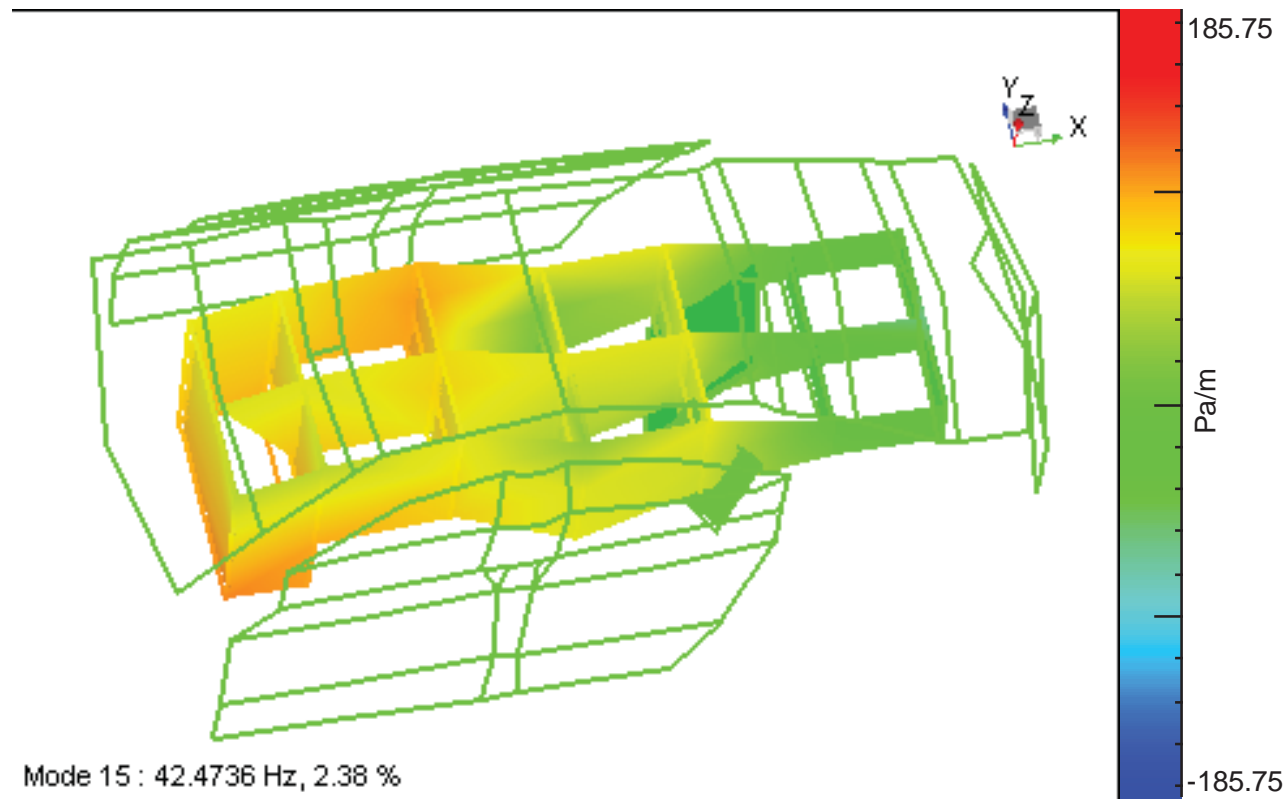

Figure 101: Vibro-Acoustic Mode at 42.5 Hz(GM Seats Speaker Closed) 
The mode shape at $42.5 \mathrm{~Hz}$ is a complex mode shape. The seats move together with a small phase lag between them. A high pressure developed in the trunk pushes the seats into the main cavity. This in turn generates high pressure in the main cavity. When a low pressure is generated in the trunk the seatbacks move inward towards the trunk.

In the final configuration, the OSB sheet covering on the package shelf speakers is removed. This would simulate the actual production condition. Figure 102 shows the vibro-acoustic mode at $39.3 \mathrm{~Hz}$. The two parts of the rear seat are completely out of phase with each other. A first acoustic mode seems to exist in the vehicle with high pressure being developed in the main cavity accompanied with low pressure generation in the trunk cavity. This is a complex mode as it is difficult to understand the causal relationship between the developed acoustic pressures and the motion of the seatback. It may so happen that the seatback is moving independently by itself without affecting or getting affected by the acoustic pressures in the vehicle.

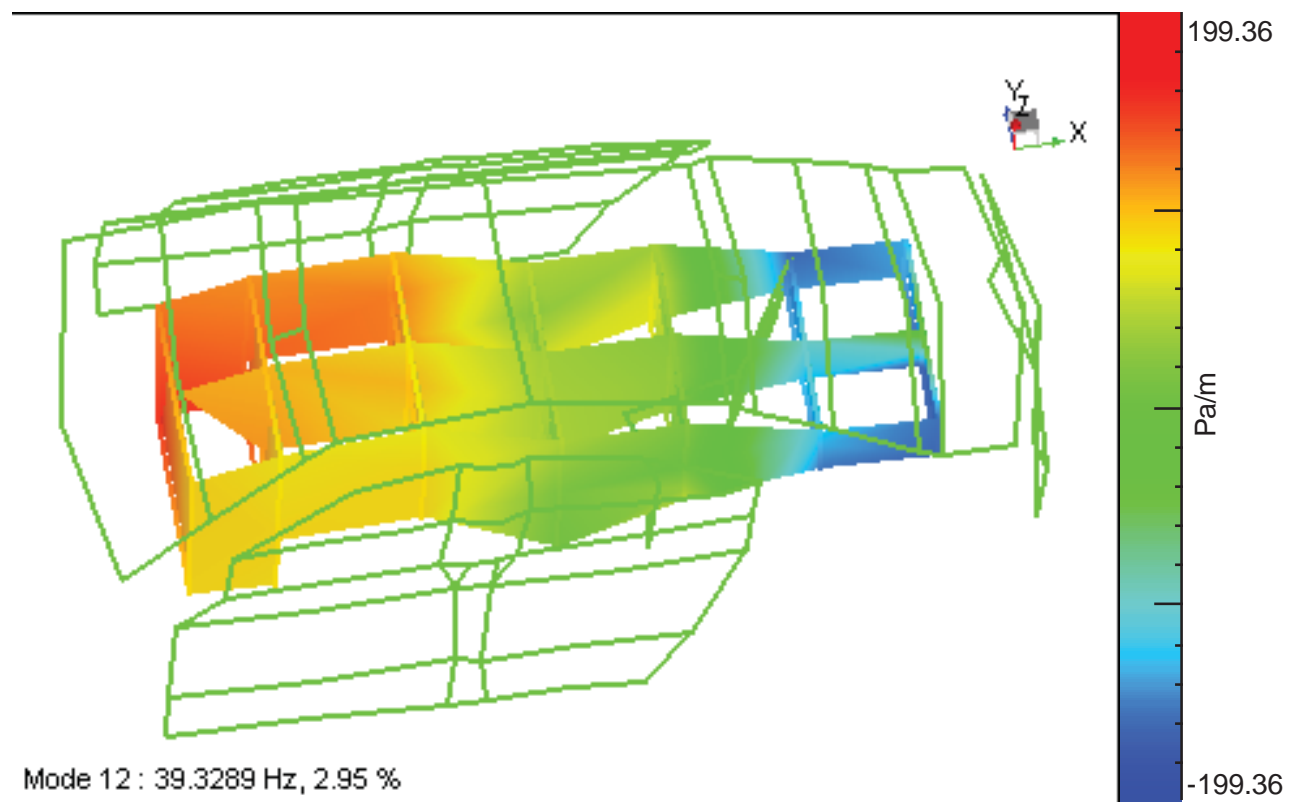

Figure 102: Vibro-Acoustic Mode at $39.3 \mathrm{~Hz}$ (GM Seats Speakers Open)

Figure 103 shows the vibro-acoustic mode at $42.6 \mathrm{~Hz}$. The two parts of the seat move in phase with each other for this mode. It seems that the acoustic pressure generated in the vehicle is driving the seatback forwards and backwards. A high pressure develops in the trunk which pushes the seatback towards the main cavity. Once a high pressure is generated in the main cavity, it pushes the seatback towards the trunk cavity. 


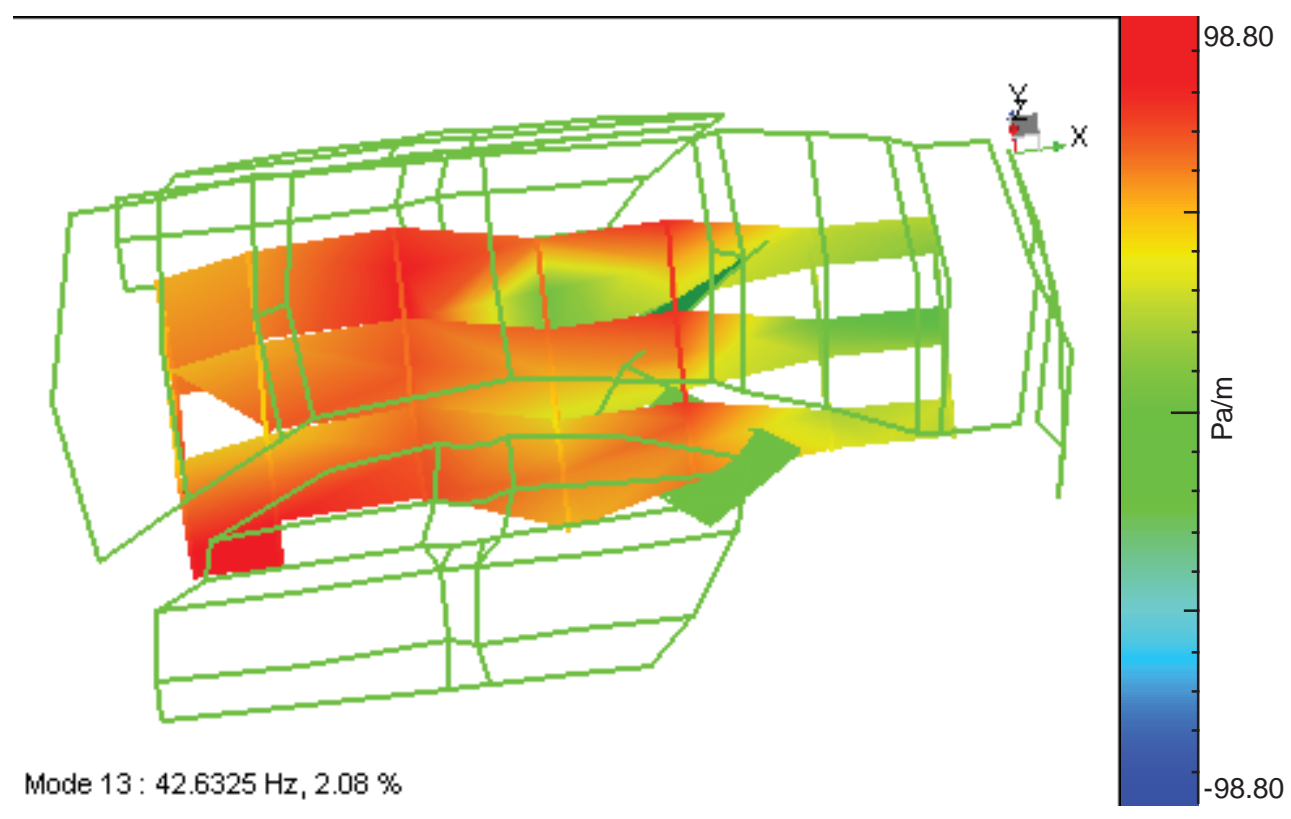

Figure 103: Vibro-Acoustic Mode at 42.6 Hz (GM Seats Speaker Open)

\subsection{Summary of Seatback Testing}

With the help of this test, information about the interaction between the structure and the fluid cavity is obtained. A very complex relation between the two media exits. Analysis of the mode shapes reveal that the interaction changes with each mode and that there is no set or defined pattern in which the motion/deformation would occur. Also, the information from these mode shapes gives us the interaction between the seatback and the vehicle cabin. This test sheds a new light on the fluid-structure interaction which helps us to understand the complex behavior between the two media. 


\section{Understanding Vibro-Acoustic behavior through Simple Car Models}

The project objective as described earlier was to correlate the results from a finite element model of the vehicle to the test results. The finite element model comprised of two parts - a structural model of the vehicle body and an acoustic model of the vehicle interior.

Figure 104 shows the full structural model of Chevrolet Cruze. Figure 105 shows the acoustic model of the vehicle. The seats and door cavities are shown in this figure. Entire acoustic model of the vehicle is shown in Figure 106. The seats are modeled as "heavy air" while the rest of the cavity is modeled as regular air.

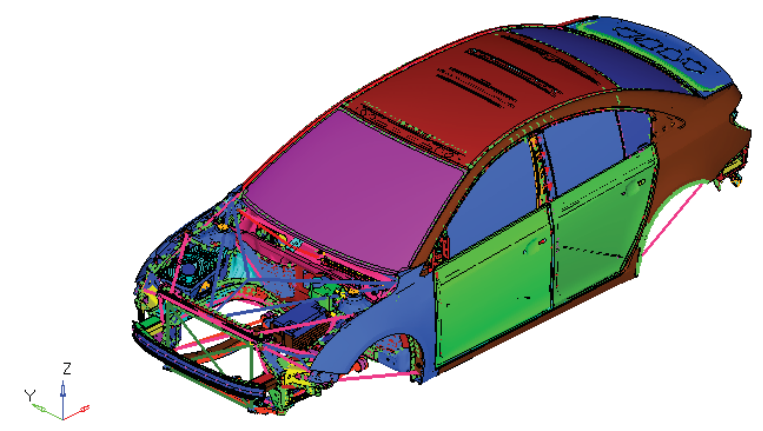

Figure 104: Structural Model of Chevrolet Cruze

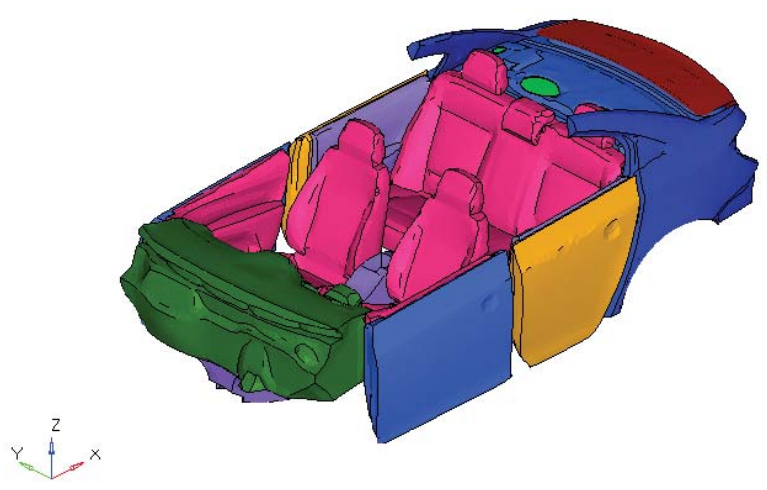

Figure 105: Acoustic Model of Chevrolet Cruze showing seats and door cavities 


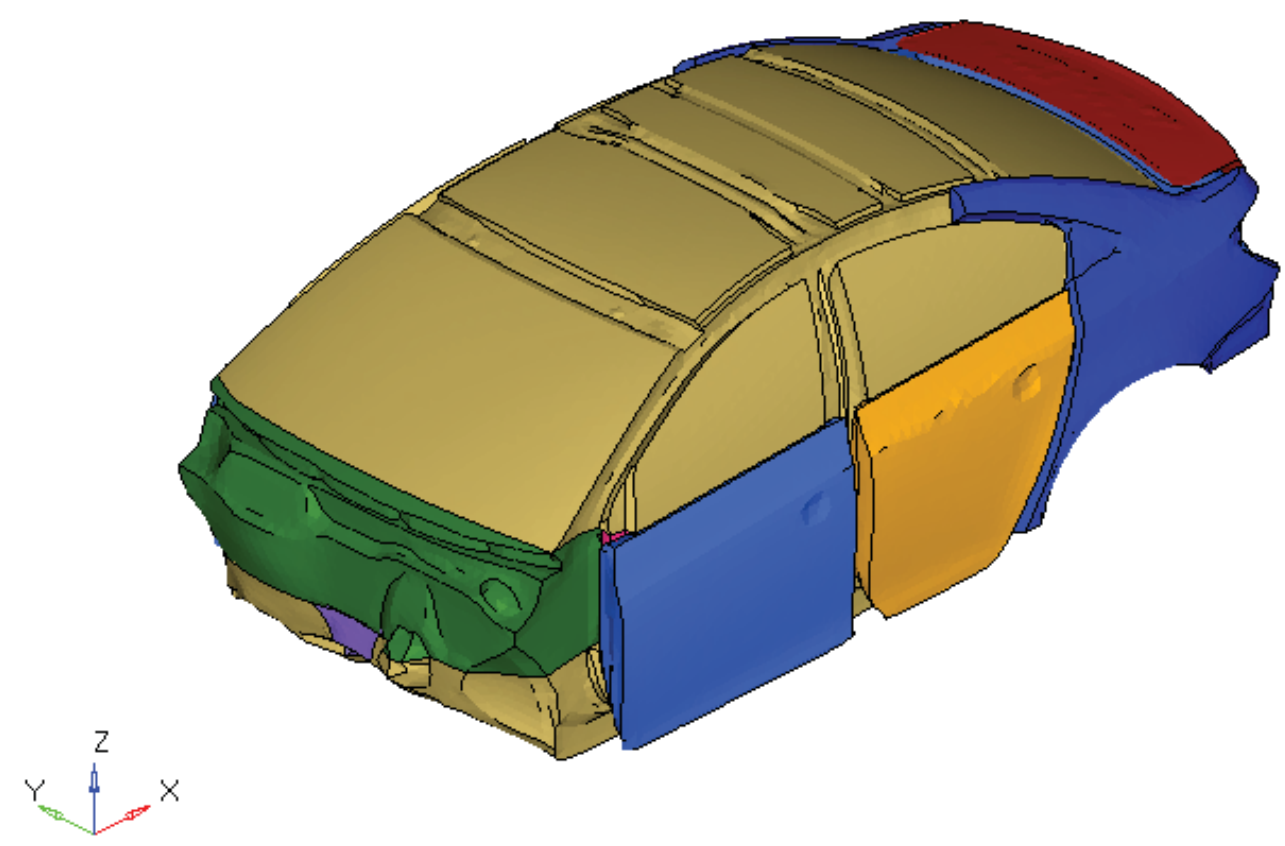

Figure 106: Complete Acoustic Model of Chevrolet Cruze

Various coupling procedures were to be tested in order to achieve the closest correlation with the test results.

Simple car shaped box models were first created and analyzed to understand the fluidstructure interaction before solving for the actual model of the vehicle. The benefit of this approach was that, the simple models were smaller in size and simpler in shape with only a few element types. Due to this, the solution time would be small. Simpler shape would make the fluid-structure interaction easy to understand. 


\subsection{Simple Car Model 1}

Figure 107 shows the dimensions of SCM 1 . The width of SCM 1 is $1000 \mathrm{~mm}$. The model is entirely made up of shell or QUAD4 elements. It has 6266 elements and 6268 nodes. Figure 108 shows the finite element model of SCM 1 with material steel having a thickness of $2.5 \mathrm{~mm}$. Figure 109 shows the cut section of the acoustic model. It consists of 82528 tetrahedral elements and 171476 nodes. Indirect Boundary Element Method (IBEM) as well as Finite Element Method was used to solve this model. BEM does not require a three-dimensional acoustic model as shown in Figure 109. A similar model as the structural model (with 2-D elements) was used in BEM. A Field Point Mesh (FPM) was also created to compute response at a rough driver's right ear (DRE) location in the model. The FPM showing the DRE location is shown in Figure 110.

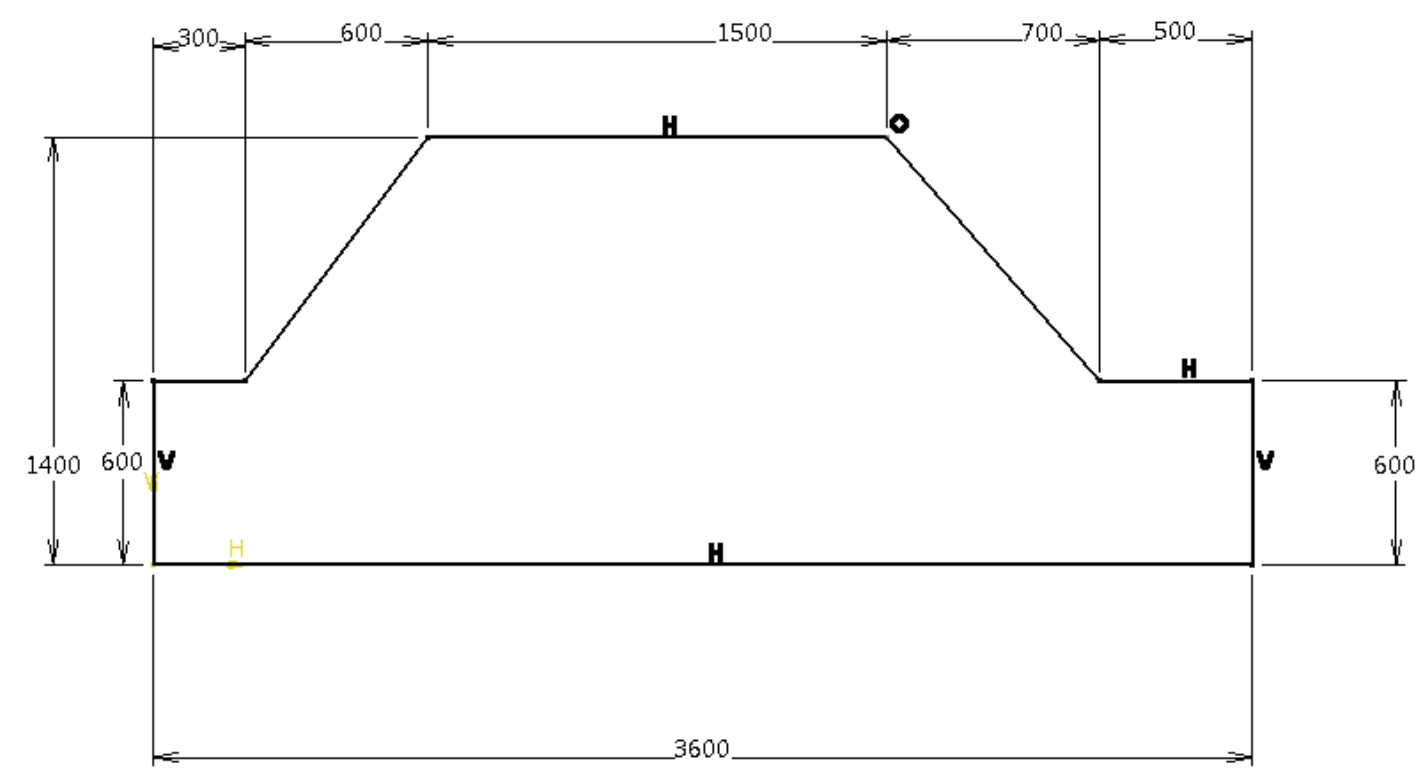

Figure 107: Dimensions of Simple Car Model 1 (in mm) 


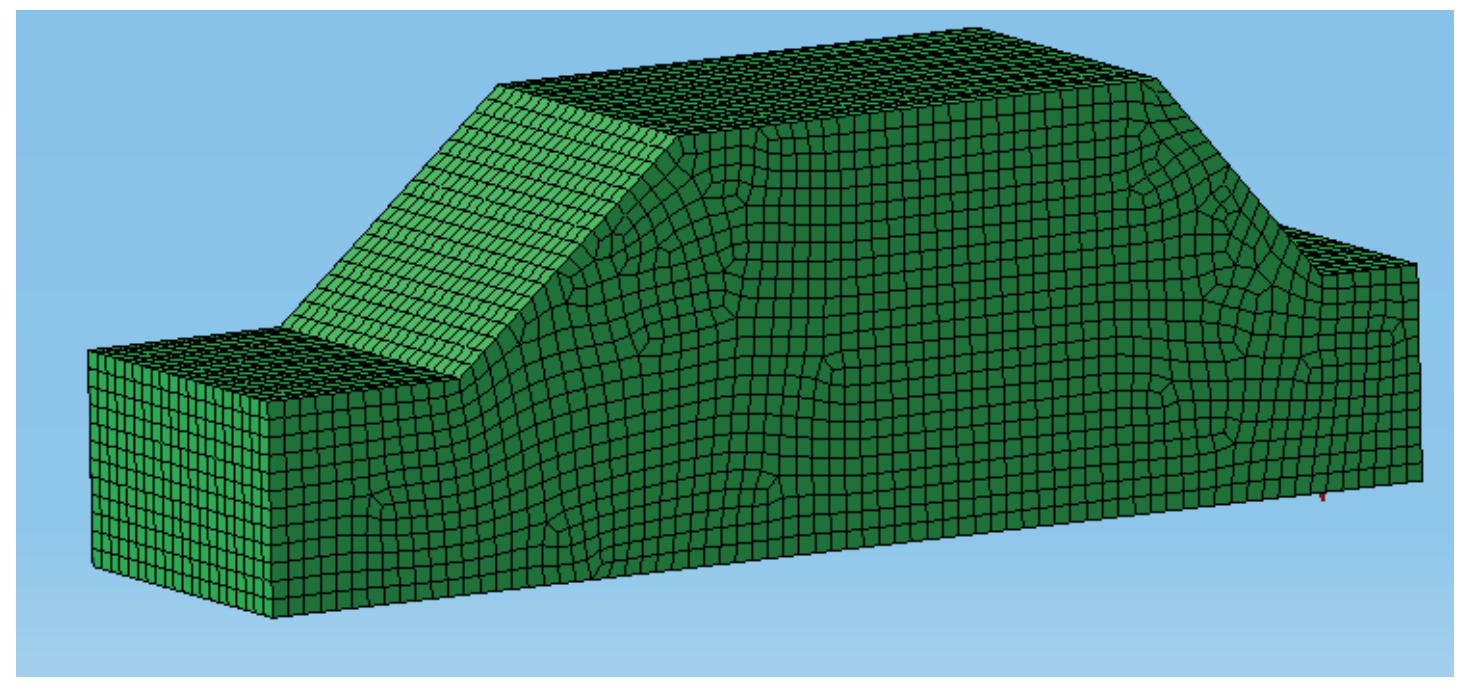

Figure 108: Structural model of SCM 1

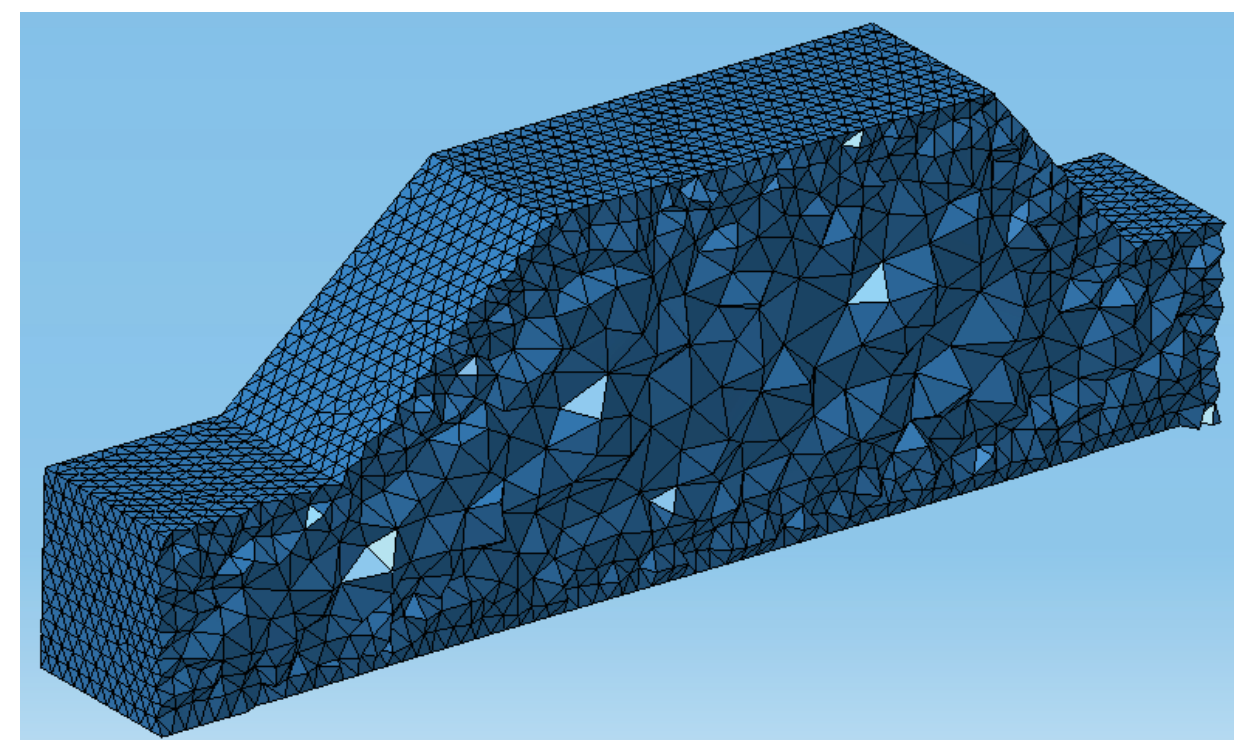

Figure 109: Cut Section of Acoustic Model for FEM 


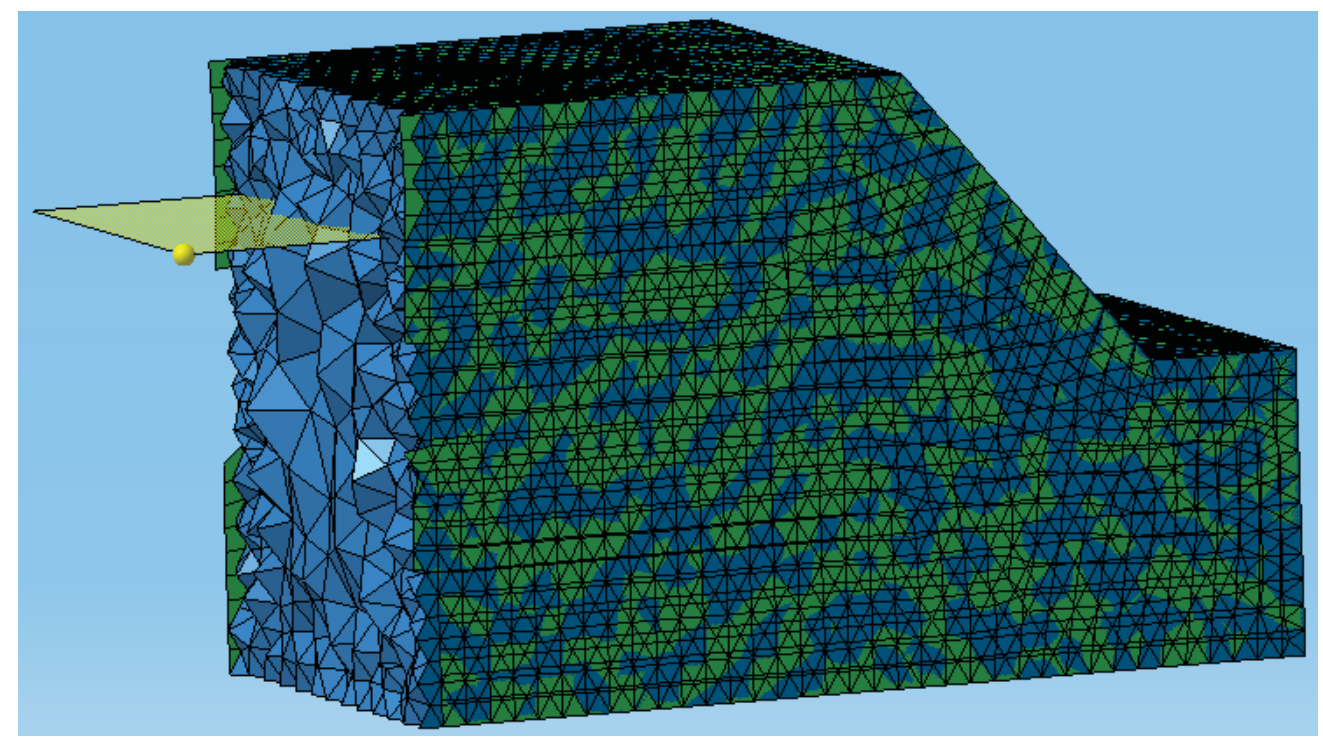

Figure 110: Cut section of entire model showing Field Point Mesh and location of DRE

Structural excitation similar to test excitation was simulated in the FEM models. Figure 111 shows the input points on the structural model.

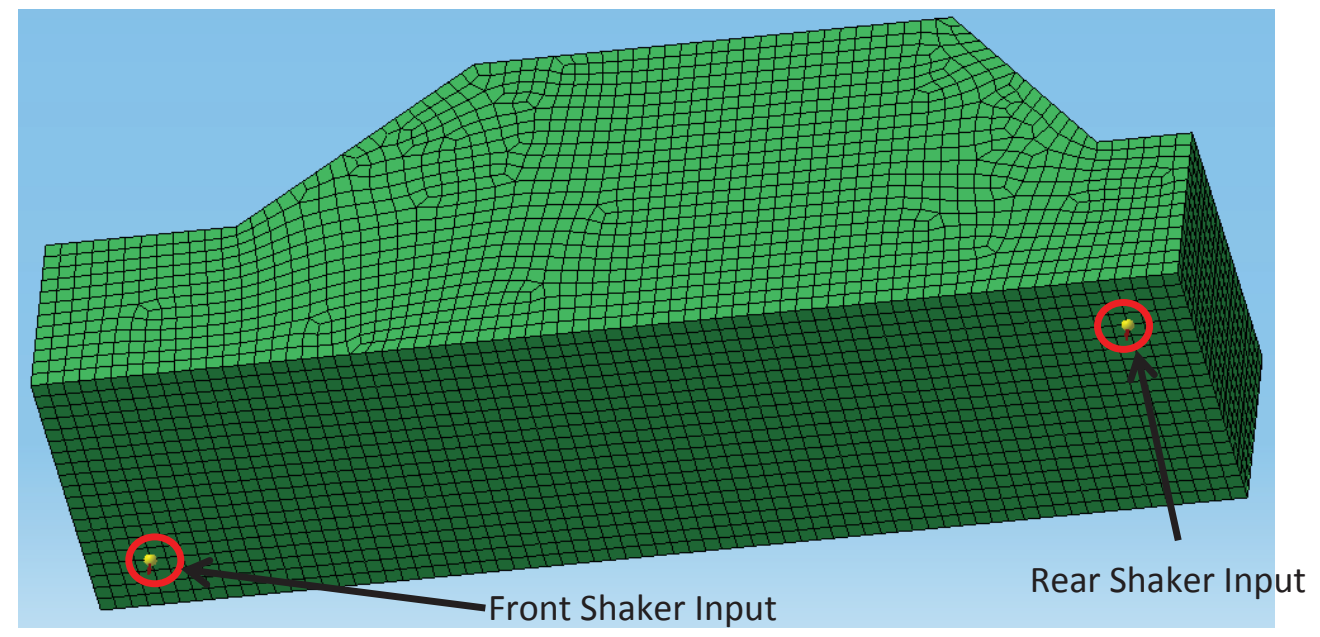

Figure 111: Simulation of Front Shaker and Rear Shaker input

Drive point FRFs are shown in Figure 112. LMS Sysnoise solver was used to solve the model for a frequency range of 0-200 Hz. The model was solved in a free-free condition. A $2 \%$ modal damping was added to the structural modes before synthesizing FRFs from them. 

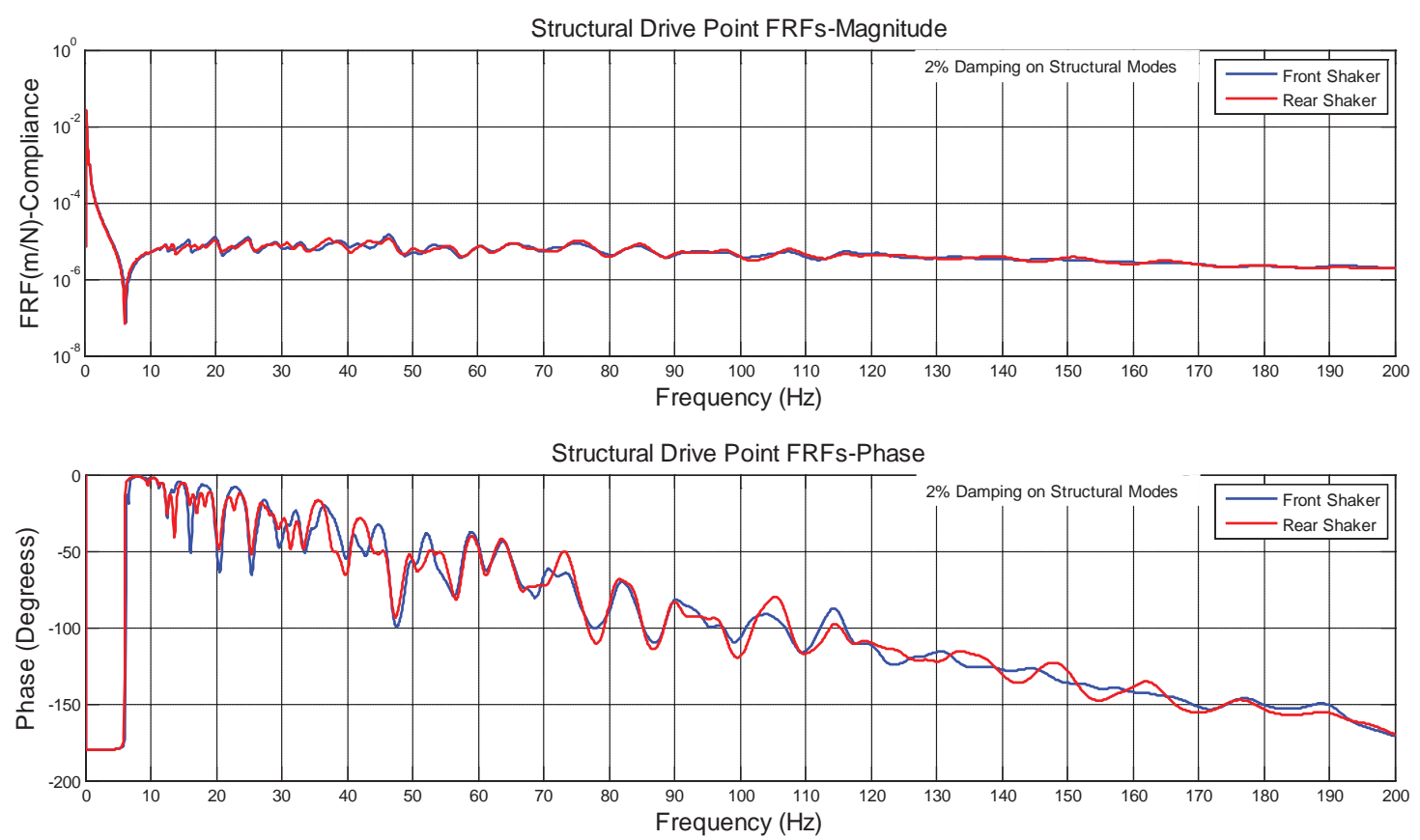

Figure 112: Drive Point FRFs of the structural inputs (Front and Rear Shaker) for SCM 1

The FRFs show similar trend in amplitude as well as phase. Due to high modal density of the model and additionally applied damping, the gain and loss in phase of the FRFs is not 180 degrees.

Figure 113 shows the acoustic sensitivity at DRE using the BEM and FEM (Uncoupled and Coupled) approach. The response due to BEM and FEM uncoupled approach is similar and the curves overlap each other till about $170 \mathrm{~Hz}$. Similarly, the response due to BEM and FEM coupled approach also overlaps for the entire frequency range.

However, the solution time for the BEM approach is much longer compared to the FEM approach as the model matrices are unsymmetrical. If the model had complex geometry or large number of elements, the solution times due to both approaches might have been comparable.

The uncoupled approach from both the methods shows a high peak at $55 \mathrm{~Hz}$. The peak due to coupled approach is $20 \mathrm{~dB}$ lower in magnitude. The first acoustic mode for the model also lies at $55 \mathrm{~Hz}$. Due to the coupling effect, the response at the DRE is much lower as compared to the uncoupled approach. 

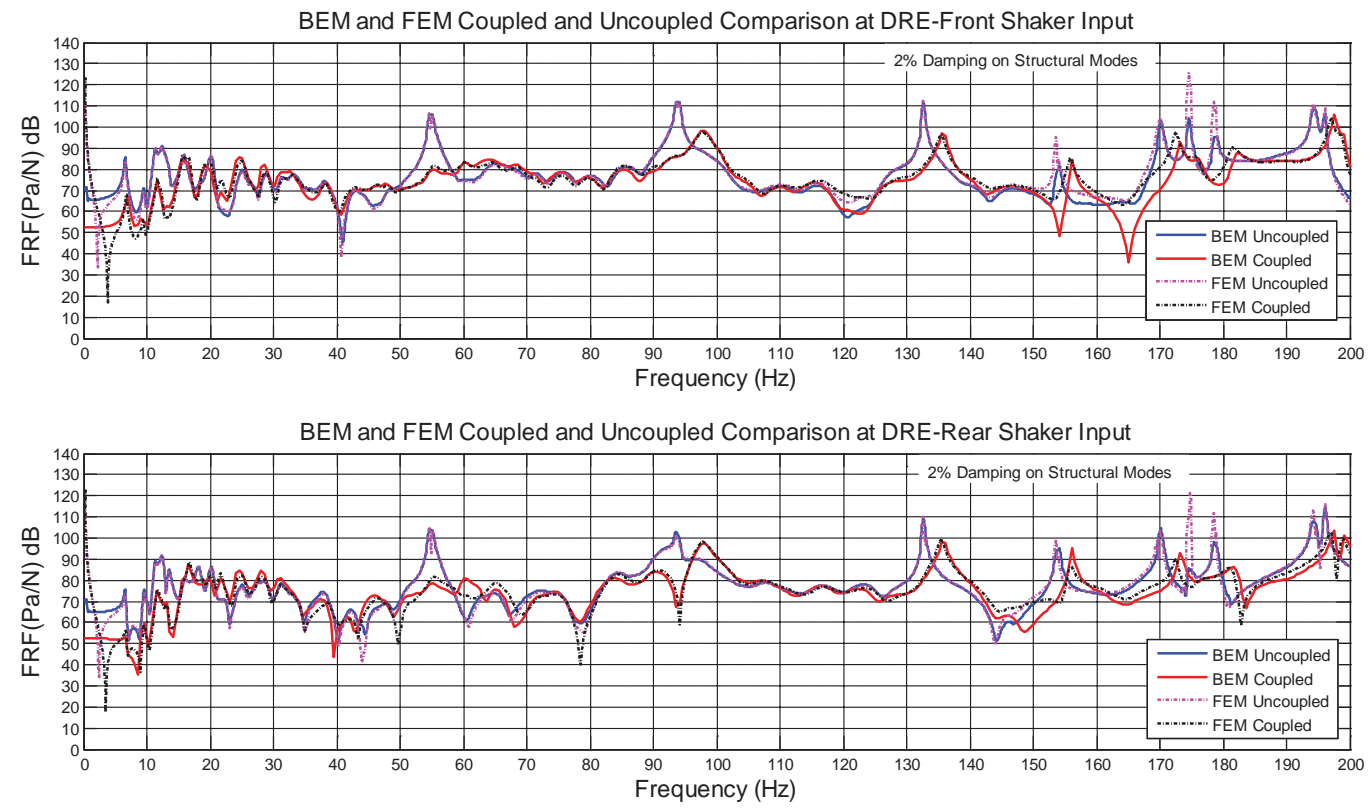

Figure 113: Acoustic Sensitivity at DRE due to structural inputs (Front and Rear Shaker) for SCM 1

Figure 114 shows structural mode and coupled structural mode at $55 \mathrm{~Hz}$ due to the BEM approach. The mode shapes are entirely different showing the effect of acoustic mode to the coupled structural mode. Figure 115 shows the participating acoustic mode at $55 \mathrm{~Hz}$.

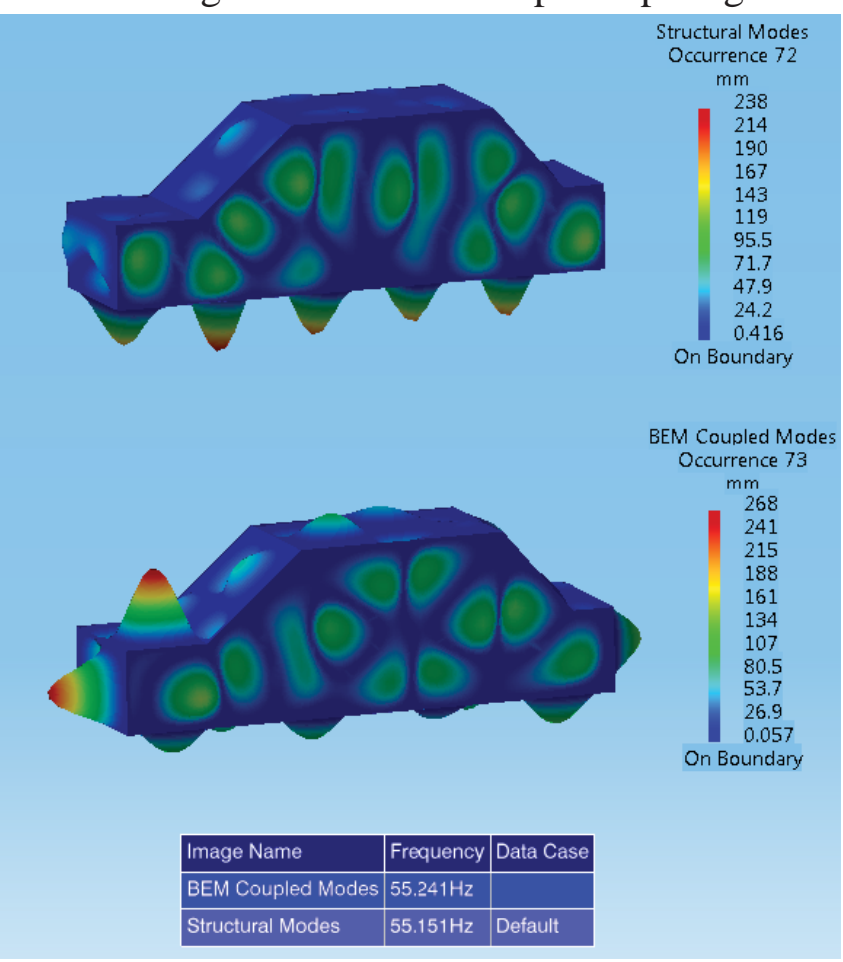

Figure 114: Structural mode and coupled mode at $55 \mathrm{~Hz}$ for BEM Case 


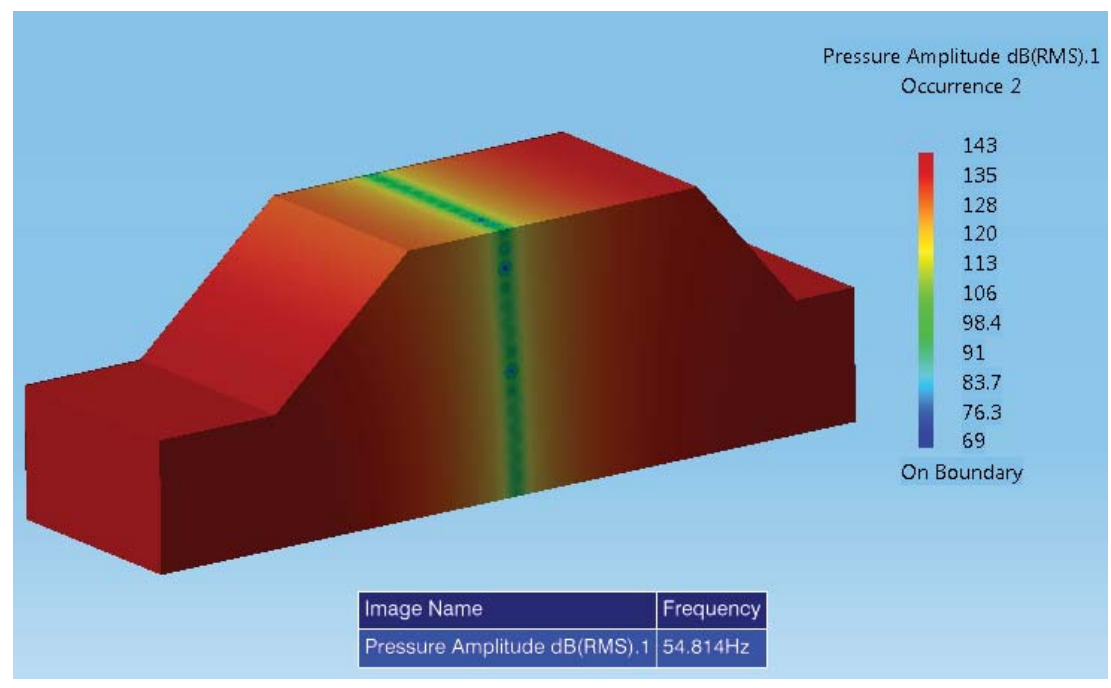

Figure 115: First Acoustic Mode at $55 \mathrm{~Hz}$ 


\subsection{Simple Car Model 2A}

Simple Car Model 2A was modeled without the engine bay compartment as in the physical experimental structure, the acoustic boom is due to the vehicle cabin and the trunk. Figure 116 shows the dimensions for SCM 2A. It's the same as SCM 1 except for the absence of the engine bay compartment.

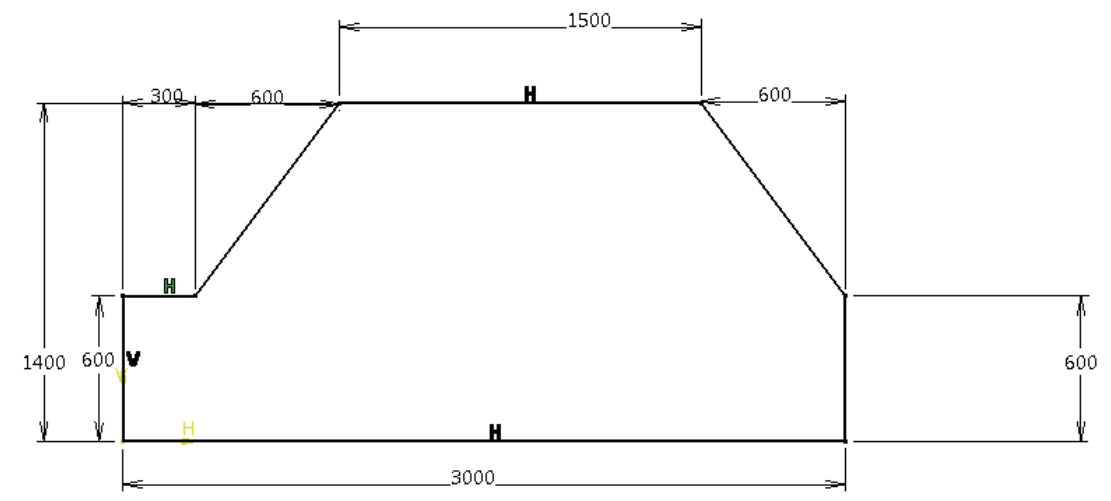

Figure 116: Dimensions of Simple Car Model 2A (in mm)

Figure 117 shows the structural mesh of SCM 2A. It was modeled to simulate A, B and C pillars of the car. Bush elements were also modeled to simulate door seals. Beams were also simulated on the roof, as the actual structural model of the Chevrolet Cruze LTZ also had them. Figure 118 shows the acoustic model of SCM 2A.
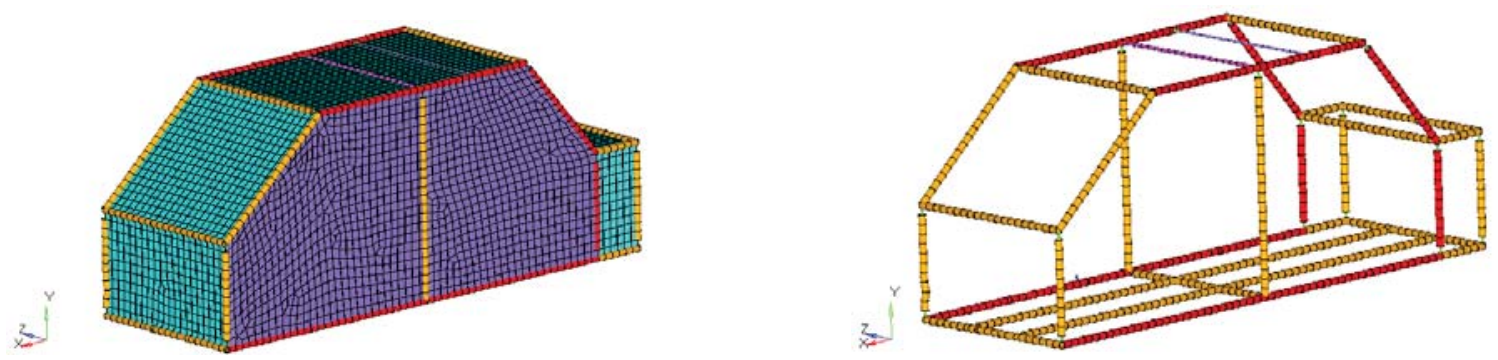

Figure 117: Left- Complete Structural Mesh of SCM2A; Right- 1-D elements in SCM $2 A$

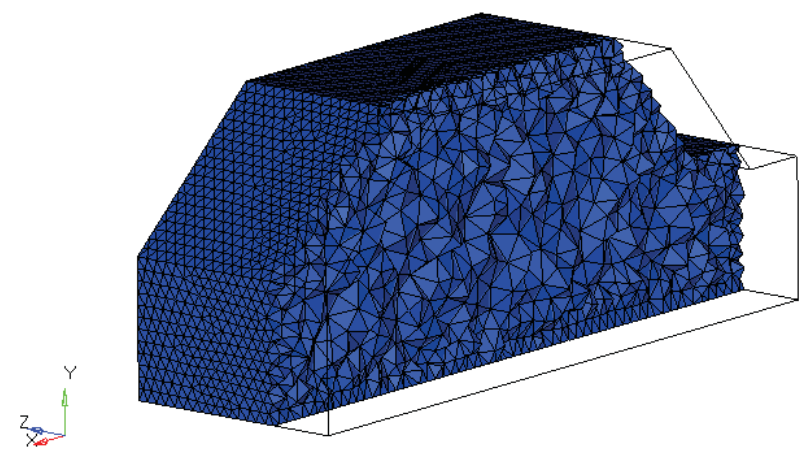

Figure 118: Acoustic Mesh of SCM 2A 
Figure 119 shows force inputs on the structural model. The location of these force inputs was chosen such that they mimic the shaker locations on the actual vehicle. Figure 120 shows the structural drive point FRFs for SCM 2A. 1\% damping was applied on the structural modes. The drive point FRFs have similar trend to the drive point FRFs of SCM 1.

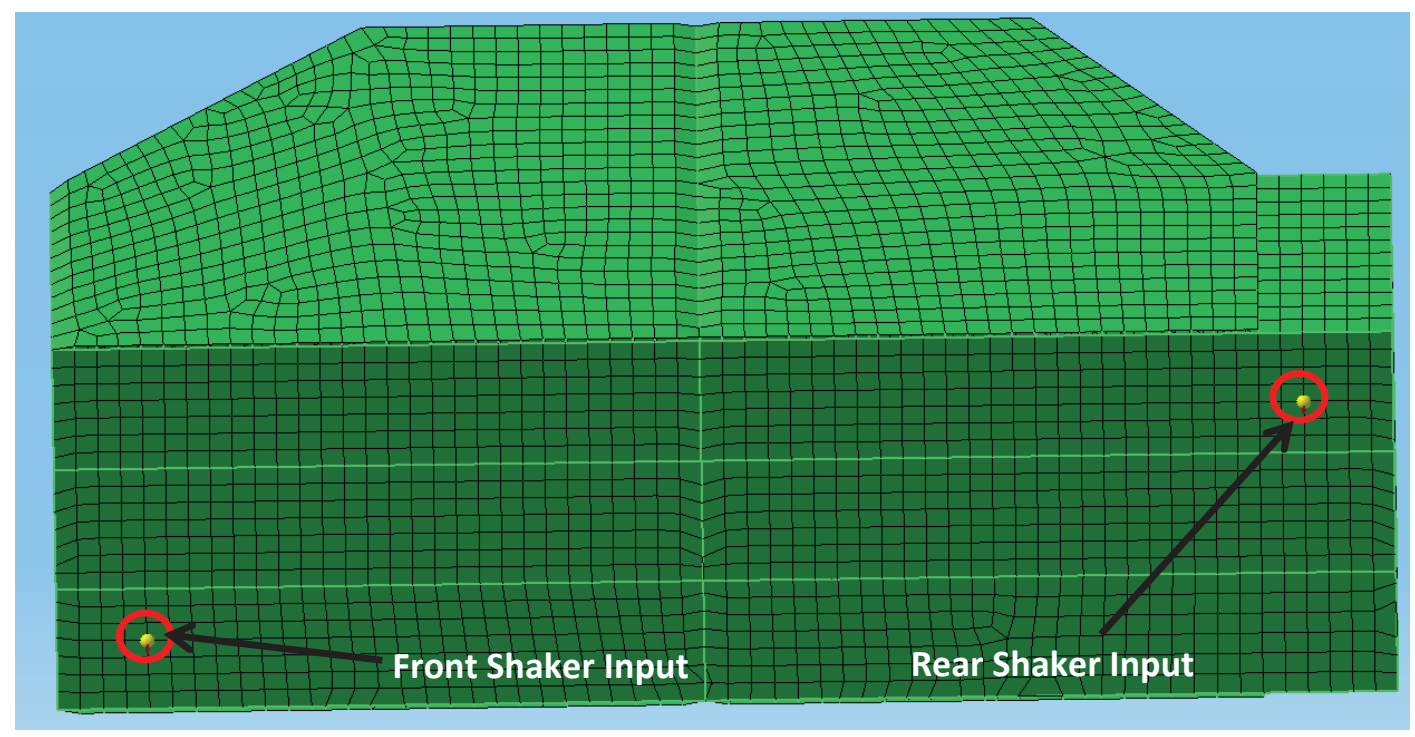

Figure 119: Simulation of Front Shaker and Rear Shaker input on SCM2A
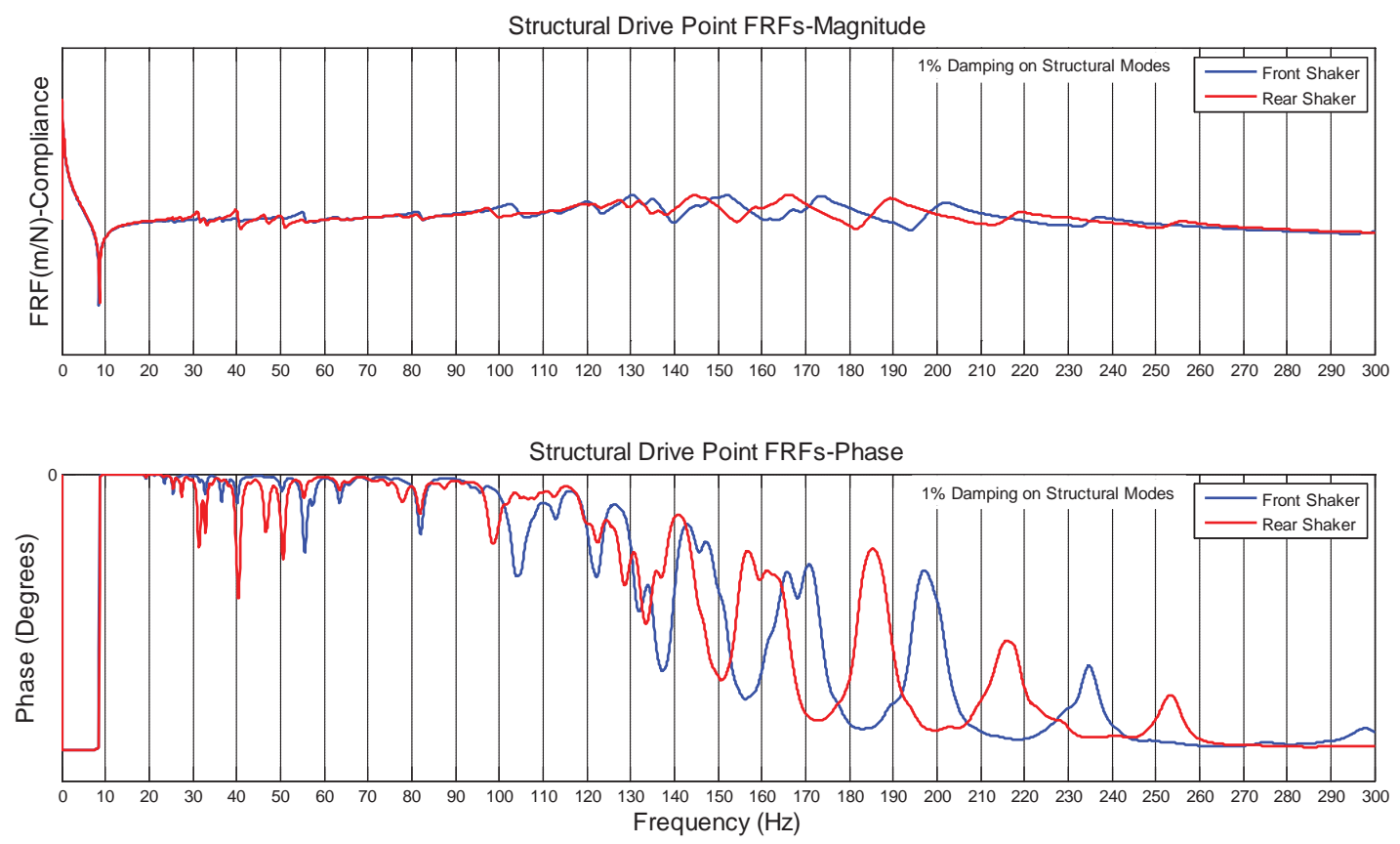

Figure 120: Drive Point FRFs of the structural inputs (Front and Rear Shaker) for SCM $2 A$ 
Figure 121 shows the acoustic sensitivity at DRE due to the structural inputs (simulated front and rear shaker). Acoustic modes for the model are also shown in the plot. Whenever an acoustic mode is encountered, a peak is seen in the acoustic sensitivity at DRE. This shows the effect of the coupling between the structural and acoustic mesh.

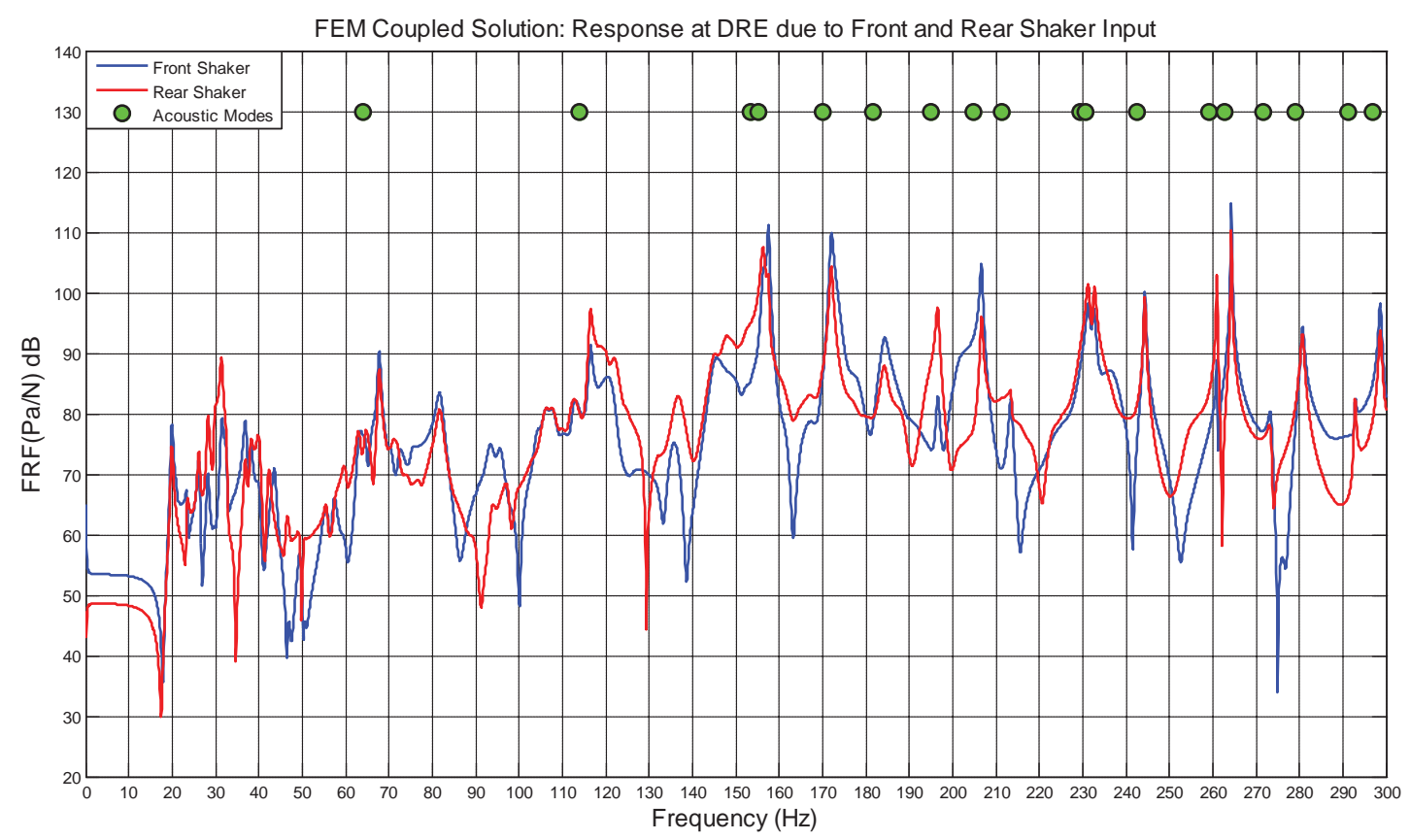

Figure 121: Acoustic Sensitivity at DRE due to structural inputs (Front and Rear Shaker) for SCM $2 A$

Acoustic MAC between the coupled acoustic modes and pure acoustic modes is shown in Figure 122. Only a couple of pure acoustic modes show a strong MAC value with the coupled acoustic modes. It can be seen that only 2 acoustic modes participate till $120 \mathrm{~Hz}$ for the coupled acoustic modes. For higher frequencies, there is a more diagonal relationship between the coupled acoustic modes and pure acoustic modes.

This also shows that, for low frequencies, the vibro-acoustic coupling will remain the same even if the structural modes are shifted by a small amount. The first few pure acoustic modes participate for a wide frequency range indicating the coupling would remain the same irrespective of small changes in the structure. 


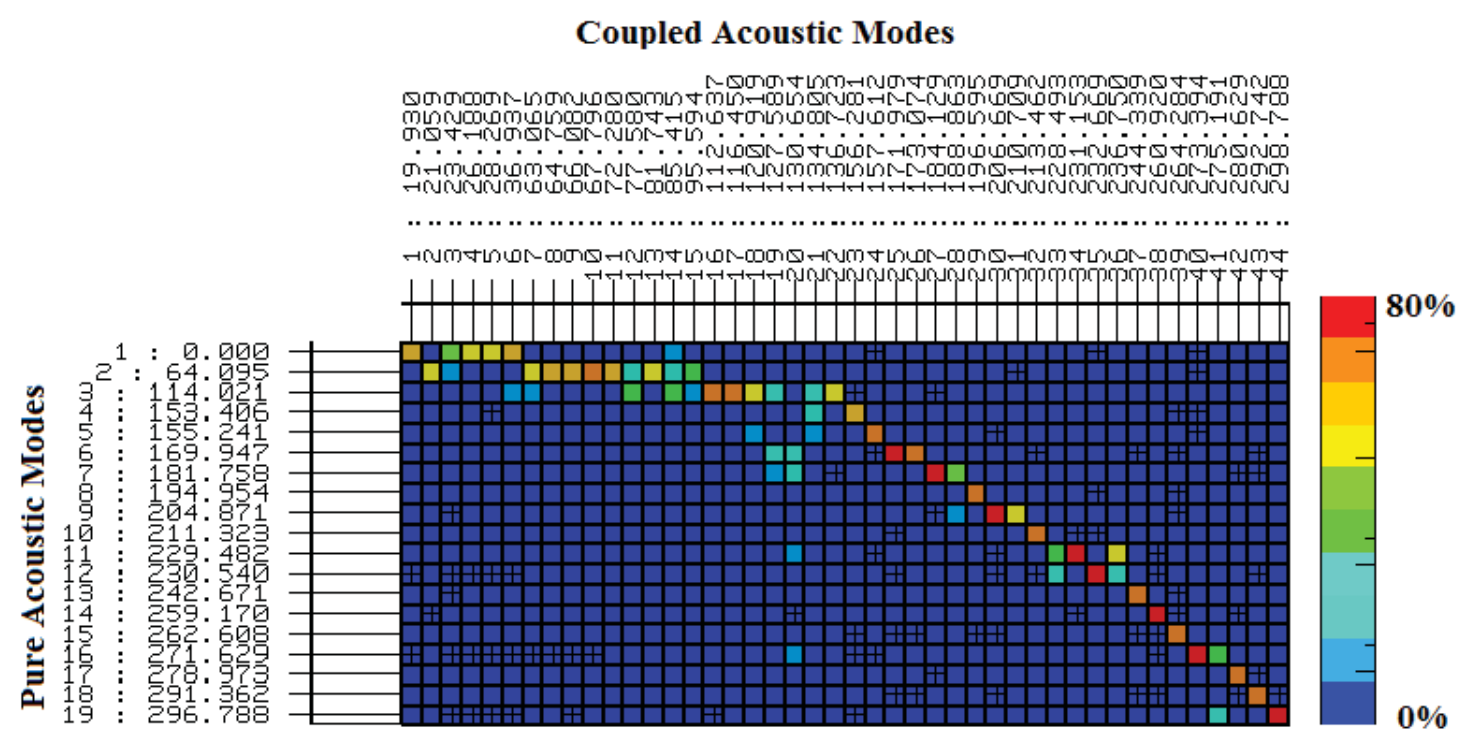

Figure 122: Acoustic MAC between Coupled Modes and Pure Acoustic Modes for SCM $2 A$ 


\subsection{Simple Car Model 2B}

In chapter 8, the discussion was about the effects of door cavities on the acoustic sensitivity of DRE. Testing was carried out with OSB sheets which completely blocked the door cavities from the main cavity (vehicle cabin cavity). SCM 2B simulates a similar condition in which the door cavities are not modeled in the acoustic model. Figure 123 shows the acoustic mesh for SCM 2B. The effective volume of the acoustic mesh is reduced due to the removal of door cavities. The structural model is the same as in SCM 2A.

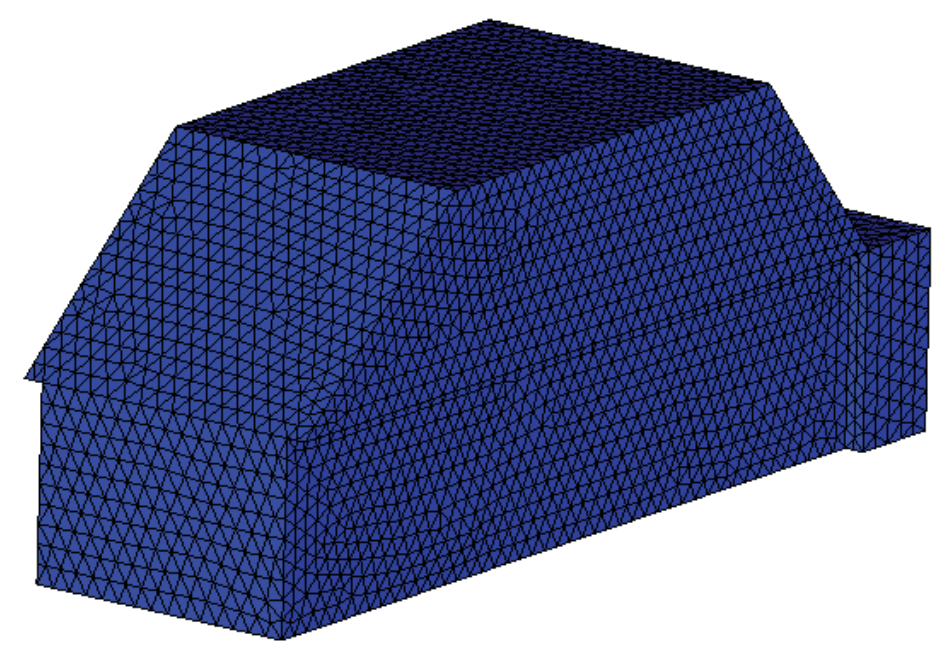

Figure 123: Acoustic Mesh for SCM 2B (model without door cavities)

Figure 124 shows the acoustic sensitivity at DRE due to the front and rear simulated inputs. The plot also shows the acoustic modes of the model. The acoustic sensitivity trend is similar to that of SCM 2A. There is some frequency shift in the acoustic modes due to reduction in volume of the acoustic model. Also, some new modes are seen in the solution. Overall, the trend of the acoustic sensitivity is quite similar to SCM 2A. This does not completely correlate with the testing results as the simulated models are very simplistic and are done so in order to understand the vibro-acoustic coupling.

Figure 125 shows the acoustic MAC between pure acoustic modes and coupled acoustic modes for SCM 2B. The MAC is also similar to SCM 2A. Only a few pure acoustic modes participate till about $120 \mathrm{~Hz}$. The $0 \mathrm{~Hz}$ mode participated till $39 \mathrm{~Hz}$. 


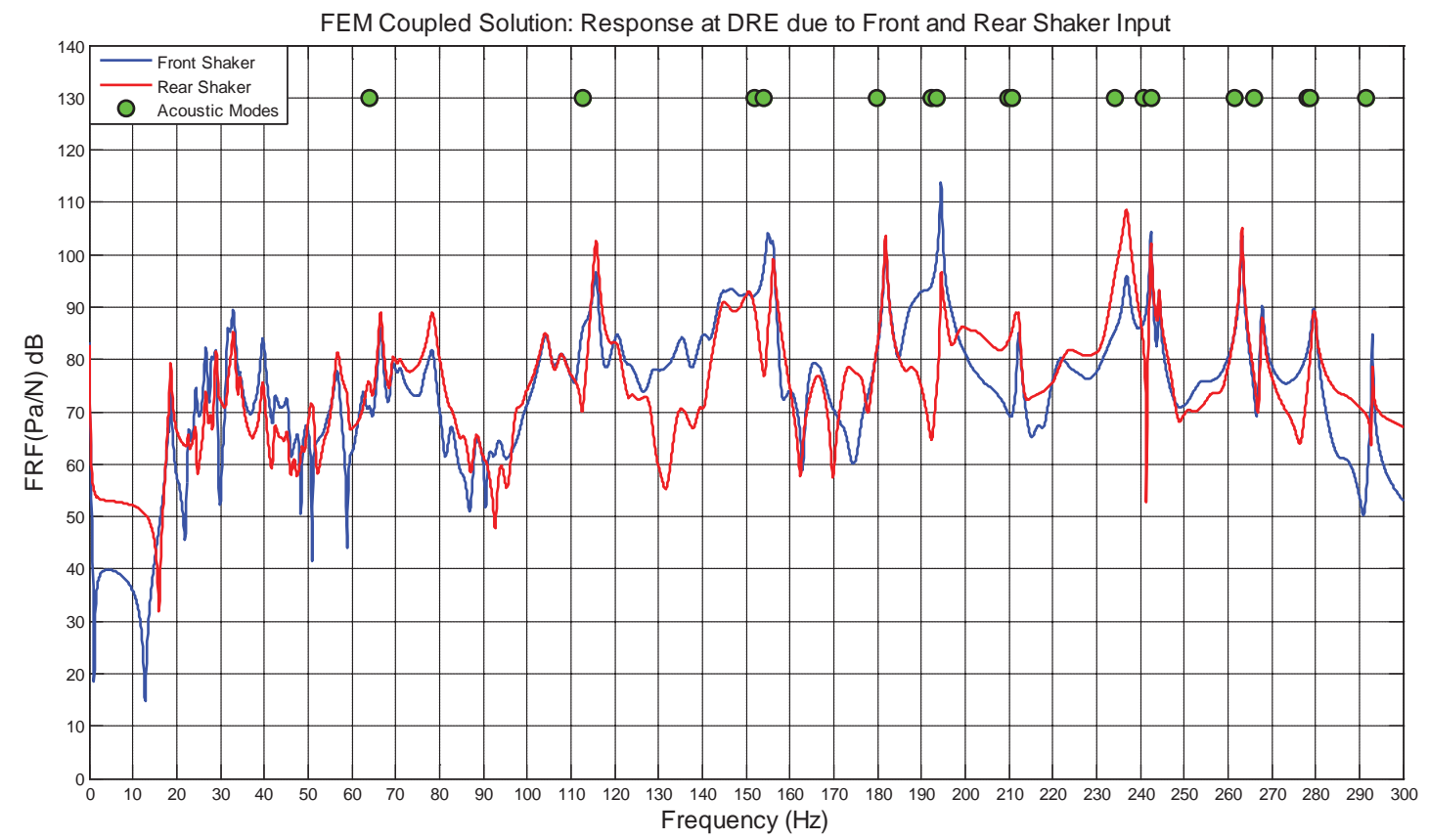

Figure 124: Acoustic Sensitivity at DRE due to structural inputs (Front and Rear Shaker) for SCM 2B

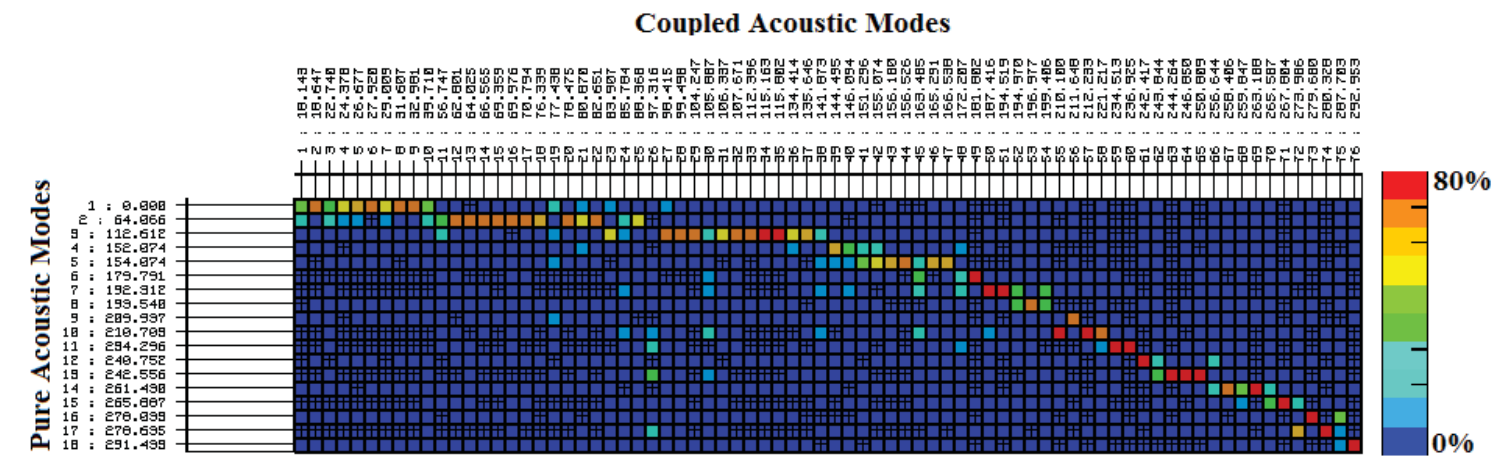

Figure 125: Acoustic MAC between Coupled Modes and Pure Acoustic Modes for SCM 2B 


\subsection{Simple Car Model 3A}

Simple Car Model 3 was modeled to understand the effect of speaker openings in the door cavities. Figure 126 shows the acoustic model for SCM 3A. The structural model is the same as SCM 2. The connection between main cavity and door cavity is shown in Figure 127. A zoomed view is shown in Figure 128.

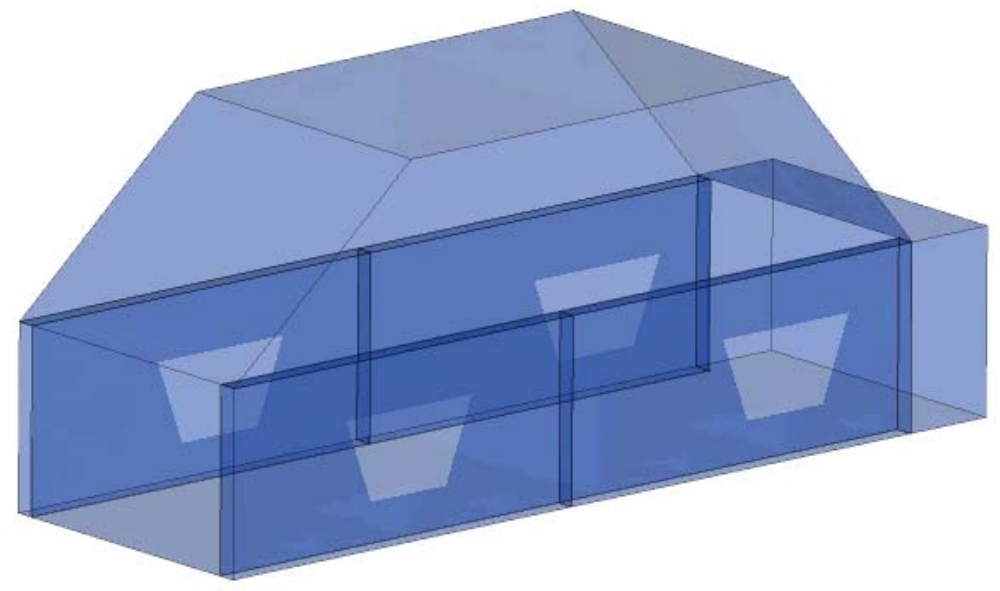

Figure 126: Acoustic Model for SCM 3A

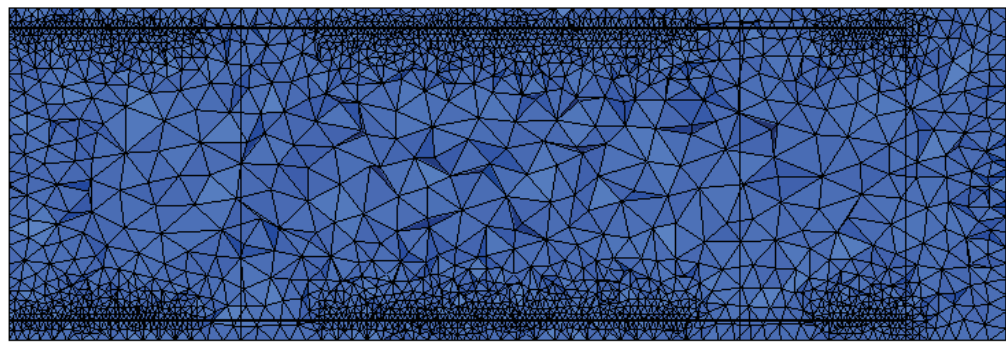

Figure 127: Bottom view of SCM 3A showing connection between main cavity and door cavities

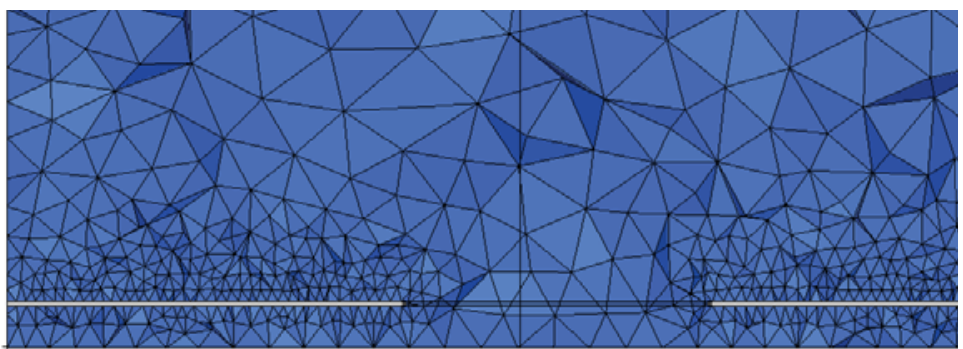

Figure 128: Zoomed view showing connection between door cavity and main cavity 
Figure 129 shows the acoustic sensitivity at the DRE along with the acoustic modes for SCM 3A. Due to the connections, the acoustic model has a lot of modes till $300 \mathrm{~Hz}$. The door cavities do behave like Helmholtz resonators, especially when they are in phase with each other.

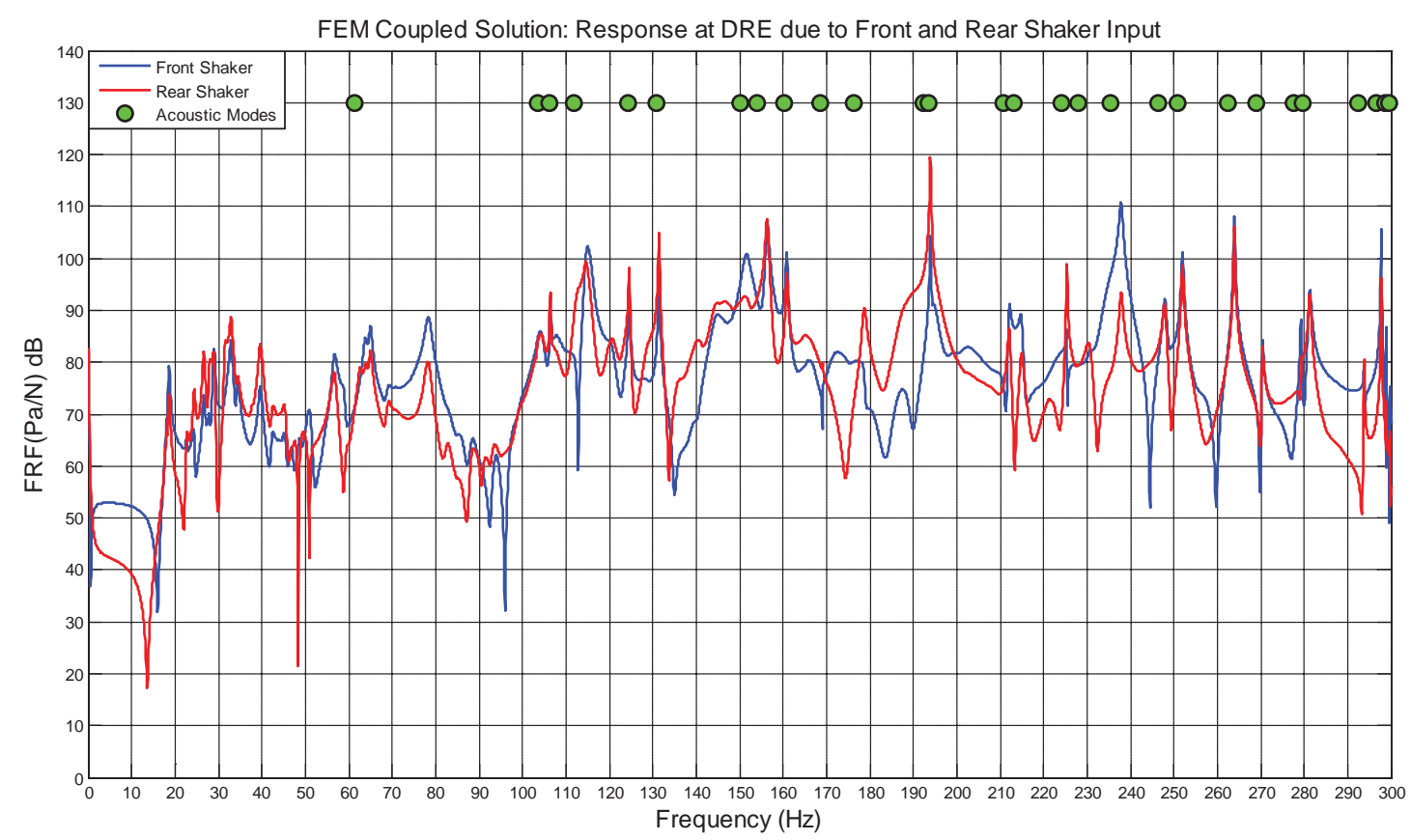

Figure 129: Acoustic Sensitivity at DRE due to front and rear shaker input for SCM $3 A$

Figure 130 shows the acoustic sensitivity comparison for SCM 2A and SCM 3A. It can be seen from the plot that SCM $3 \mathrm{~A}$ has a lot of modes as compared to SCM 2A. The response for SCM 3A is much lower between $170-190 \mathrm{~Hz}$ when the input is due to front and rear shaker. Also, comparing the input due to the rear shaker, the response for SCM $3 \mathrm{~A}$ is lower between $60-90 \mathrm{~Hz}$. 


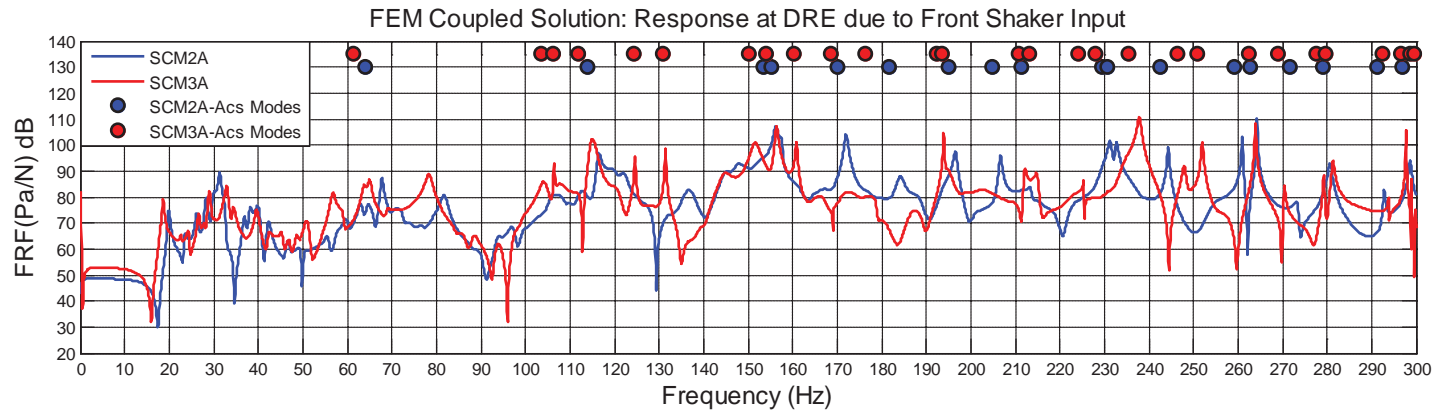

FEM Coupled Solution: Response at DRE due to Rear Shaker Input

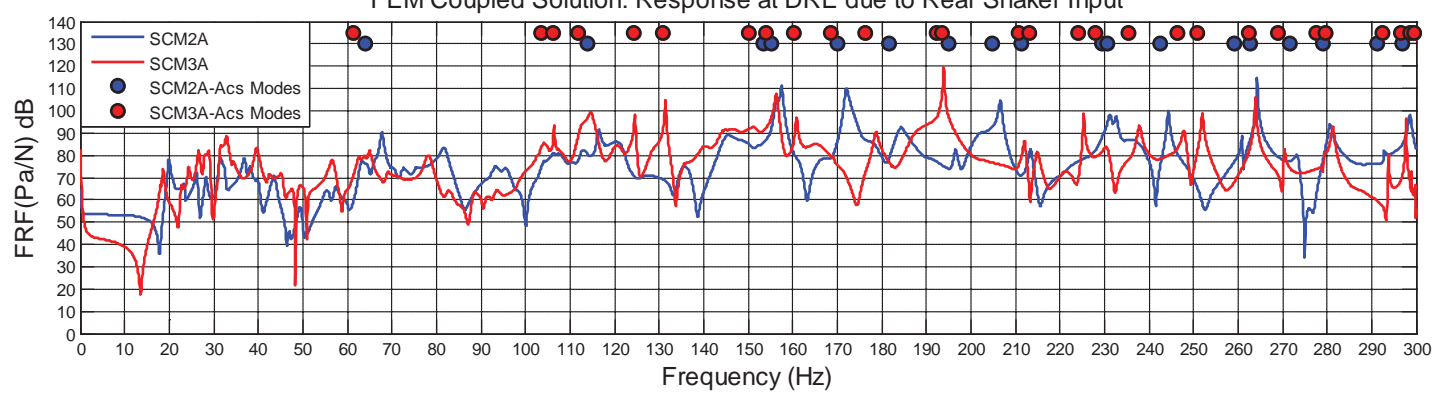

Figure 130: Comparison of Acoustic Sensitivity at DRE for SCM 2A and SCM 3A

Comparison of modes between pure acoustic modes and coupled acoustic modes is shown in the acoustic MAC in Figure 131. The $0 \mathrm{~Hz}$ mode participates till $40 \mathrm{~Hz}$ for coupled acoustic modes while the $61 \mathrm{~Hz}$ pure acoustic mode participates from 40-100 Hz. The trend of the MAC is similar to the earlier ones shown for the previous simple car models.

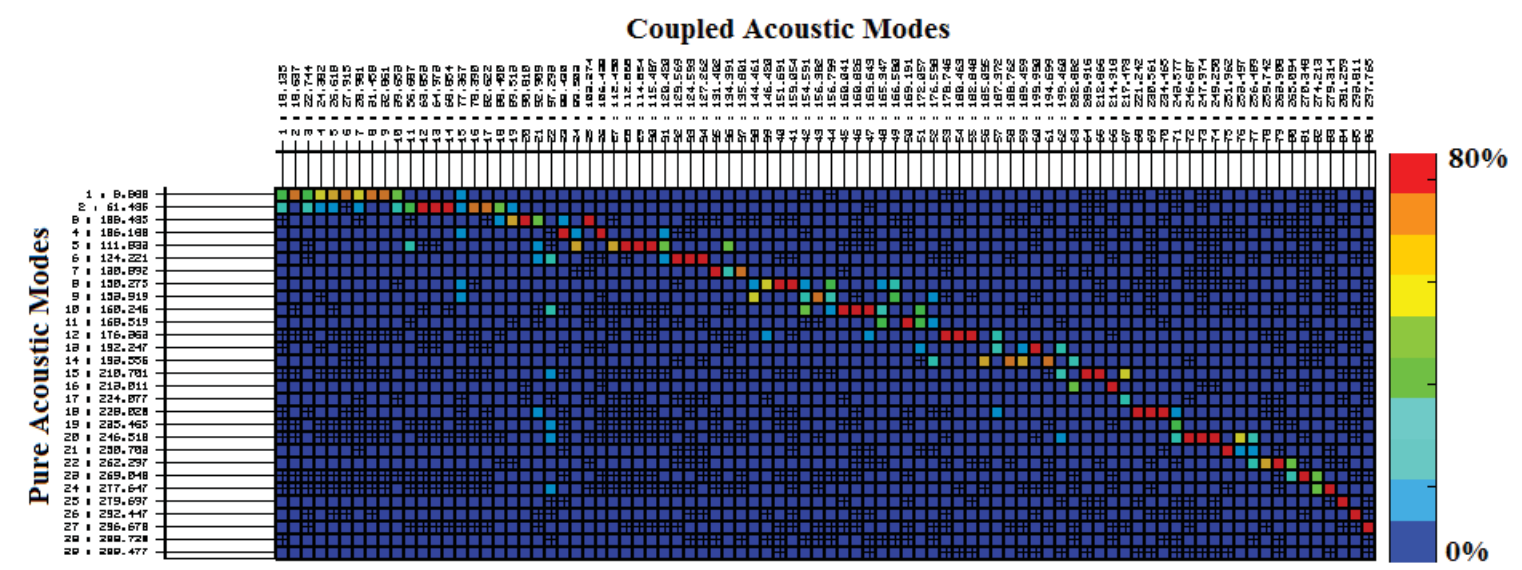

Figure 131: Acoustic MAC between Coupled Modes and Pure Acoustic Modes for SCM $3 A$ 


\subsection{Simple Car Model 3B}

In the case of SCM 3B, the number of openings were increased but the total area of opening was kept the same. The area of opening between the main cavity and the door cavities was the same. The acoustic model for SCM 3B is shown in Figure 132. The structural model is the same as for the earlier models.

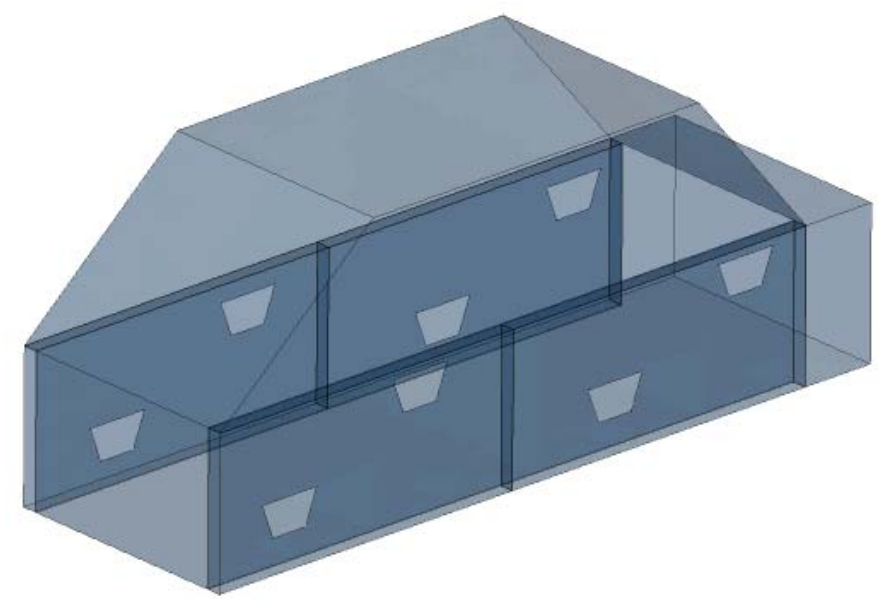

Figure 132: Acoustic Model for SCM 3B

Figure 133 shows the acoustic sensitivity at DRE for SCM 3B. Similar to the earlier models, the peaks in the sensitivity are seen when the acoustic modes are encountered. The trends are similar to SCM3A. However, the frequency of acoustic modes changes above $100 \mathrm{~Hz}$. This is due to the additional opening for each door cavity. Now, the door cavities will not behave like Helmholtz resonators due to the two openings.

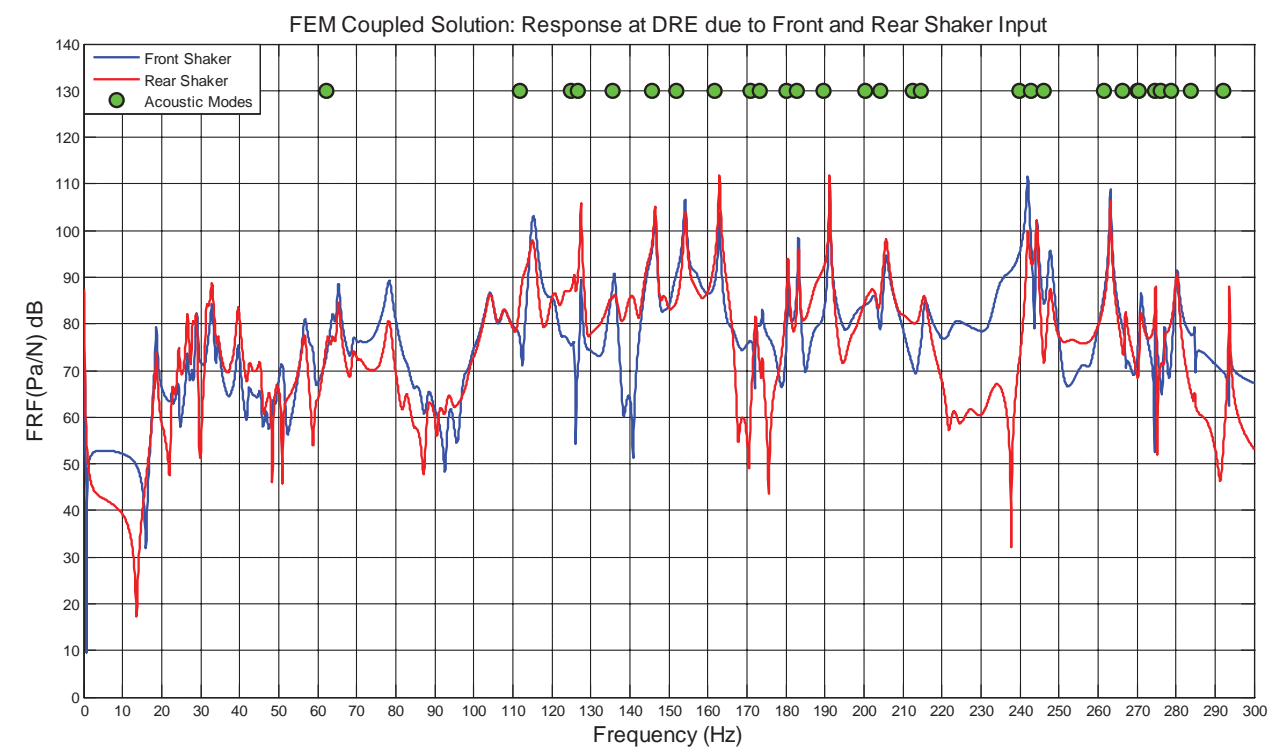

Figure 133: Acoustic Sensitivity at DRE due to front and rear shaker input for SCM 3B 
Acoustic MAC for SCM 3B is shown in Figure 134. The $0 \mathrm{~Hz}$ pure acoustic mode participates till $40 \mathrm{~Hz}$ for the coupled acoustic modes. Very high values of MAC are seen from the second pure acoustic mode. Almost all of them are above $80 \%$.

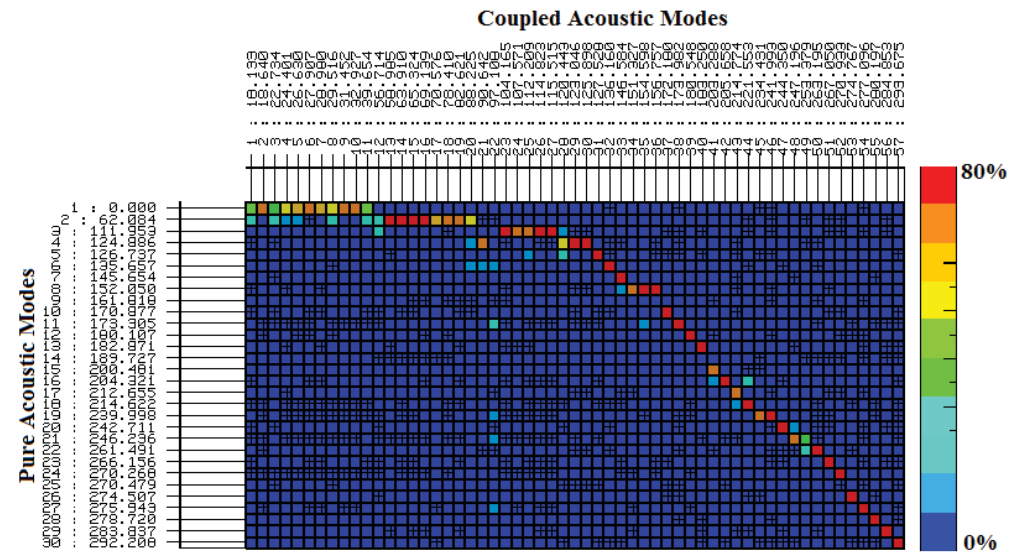

Figure 134: Acoustic MAC between Coupled Modes and Pure Acoustic Modes for SCM 3B

The only difference between SCM3A and SCM3B is the additional opening for each door cavity. The total area of opening is the same; however, the placement of the openings is also different. However, the placement of the opening should not matter as the geometries are simple. Figure 135 shows the acoustic sensitivity comparison between SCM3A and SCM3B. The curves overlap each other till $100 \mathrm{~Hz}$ where only one pure acoustic mode participates. The frequency difference is small for the first pure acoustic mode between SCm3A and SCM3B. Above $100 \mathrm{~Hz}$, as the acoustic modes change in frequency, a change in the sensitivity at the DRE is also observed.
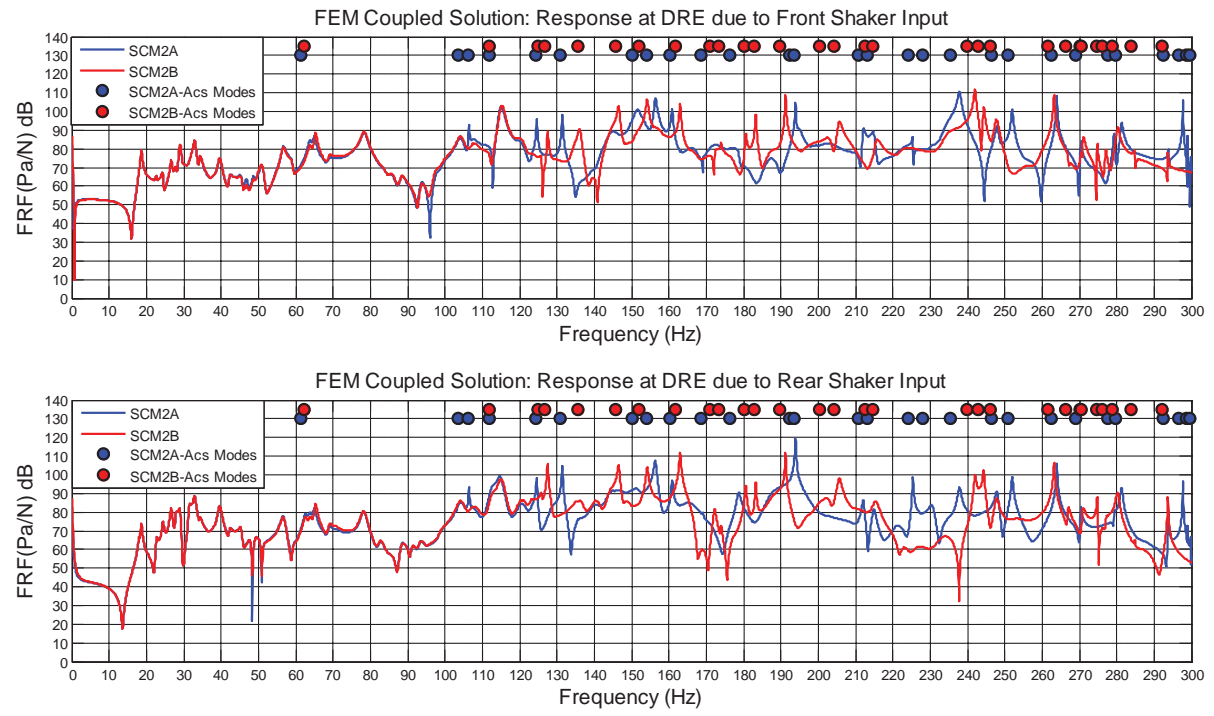

Figure 135: Acoustic Sensitivity Comparison between SCM3A and SCM3B 


\subsection{Simple Car Model 4A}

Simple car model 4 was modeled to understand the effect of rear seats and speaker openings on the rear package shelf. As mentioned in the beginning of Chapter 10, the rear seats are modeled as "heavy air". This is modeled as having a speed of sound of $150 \mathrm{~m} / \mathrm{s}$ and density of $60 \mathrm{~kg} / \mathrm{m}^{3}$. Figure 136 shows the acoustic model for SCM4A. The structural model remains the same as for the earlier models. The figure shows three sections: main cavity, rear seats and the trunk cavity. The main cavity and trunk cavity are modeled as regular air.

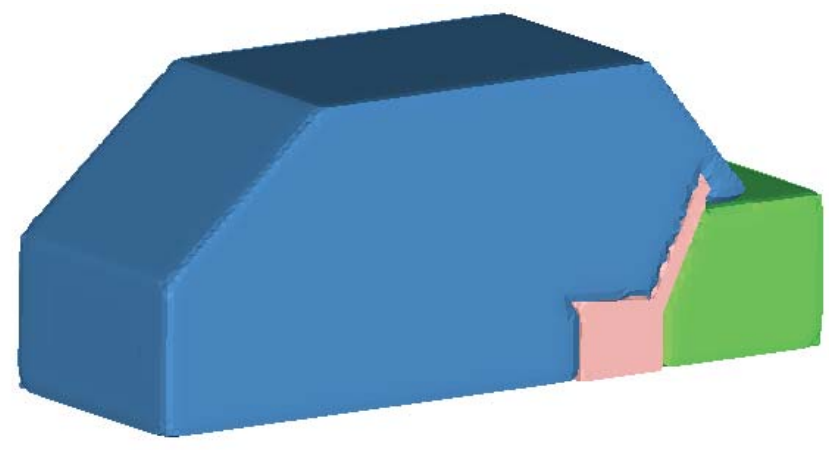

Figure 136: Acoustic Model for SCM4A

Figure 137 shows the acoustic sensitivity at DRE due to the simulated front and rear shaker inputs. Due to the introduction of rear seats, the pure acoustic modes drop in frequency. The first mode is at $34 \mathrm{~Hz}$ while the second mode is at $80 \mathrm{~Hz}$.

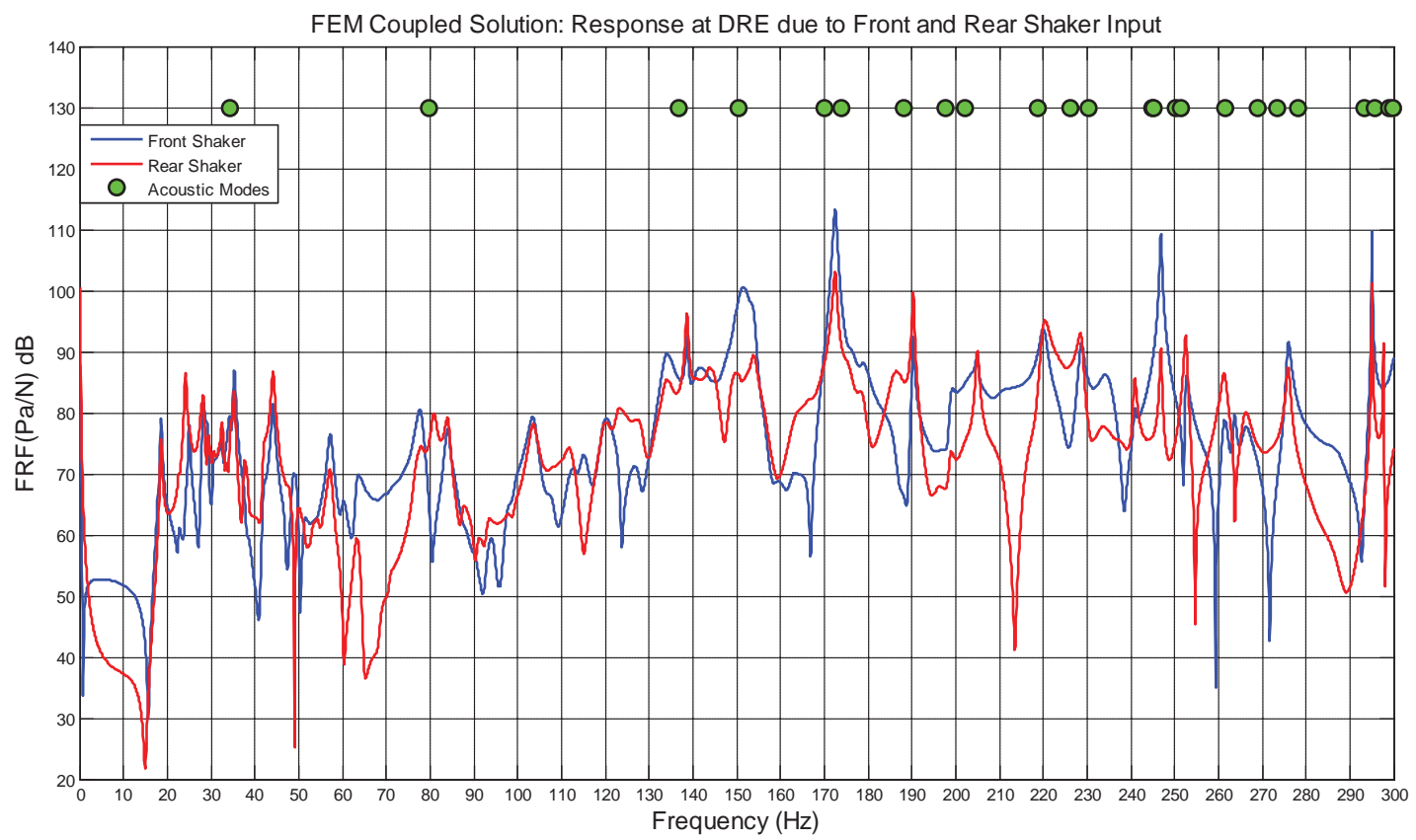

Figure 137: Acoustic Sensitivity at DRE due to front and rear shaker input for SCM 4A 
Figure 138 shows the acoustic MAC between pure acoustic modes and coupled acoustic modes for SCM4A. Compared to the earlier models, the MAC values for this model are not high. This is due to the introduction of seats in the model. However, the general trend of the MAC remains unchanged.

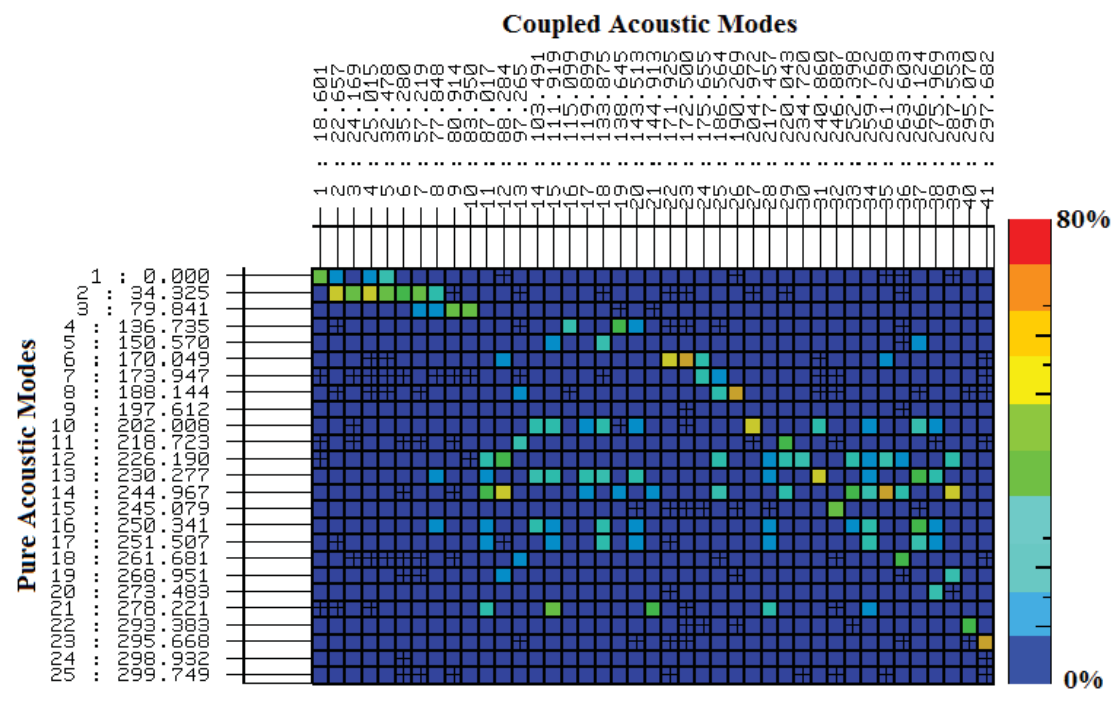

Figure 138: Acoustic MAC between Coupled Modes and Pure Acoustic Modes for SCM 4A 


\subsection{Simple Car Model 4B}

SCM4B is modeled to understand the effect of having speaker holes in the rear package shelf. The speaker openings are acoustically transparent and allow the acoustic fluctuations to travel between the main cavity and trunk cavity. Thus, as we had seen in the test structure, the trunk can pump air from the rear which would affect the acoustic response at the DRE. Figure 139 shows the acoustic model for SCM4B. The rear speaker opening can be seen. Figure 140 shows the top view of the acoustic model. The speaker openings can also be seen through the figure.

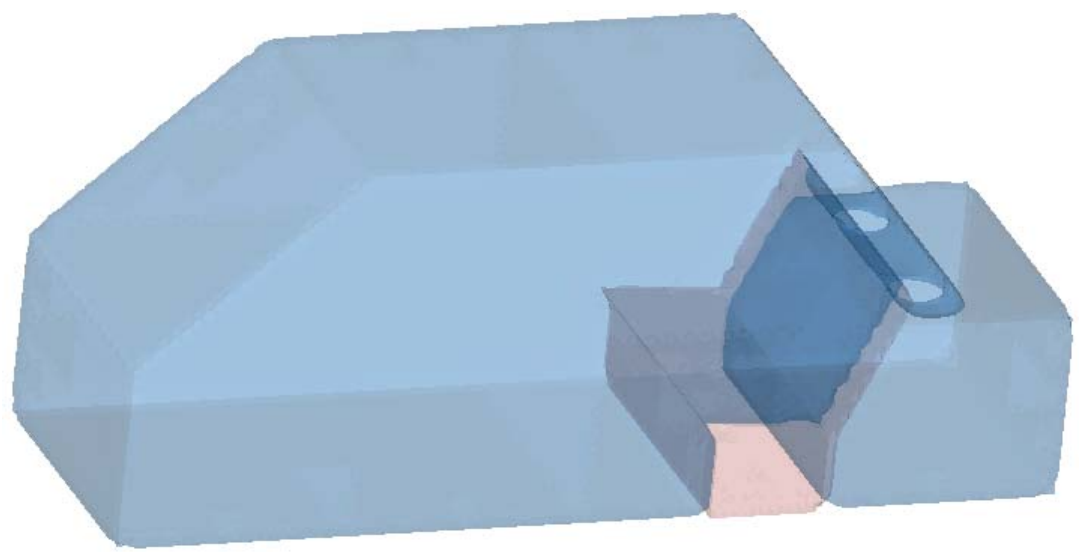

Figure 139: Acoustic Model of SCM 4B

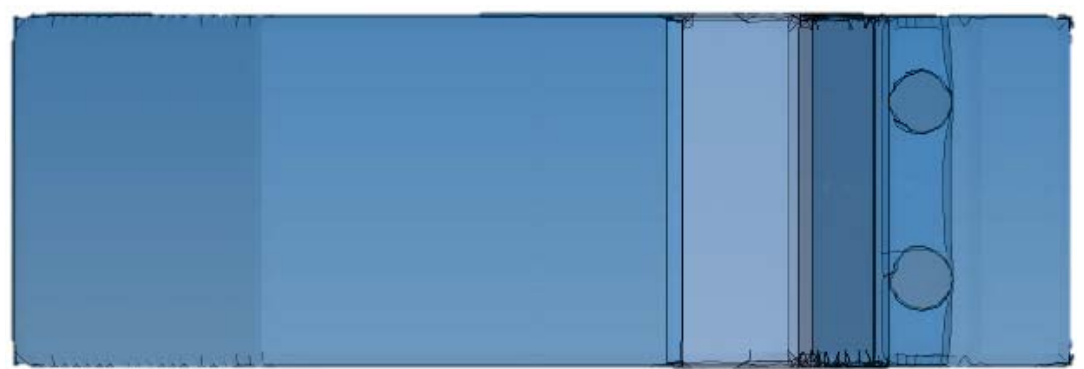

Figure 140: Top View of SCM 4B showing the speaker openings

Figure 141 shows the acoustic sensitivity at DRE for SCM4B. The frequencies of acoustic modes are also shown in the plot. Compared to SCM4A, the frequencies of acoustic modes shift considerably higher for SCM4B. To explain this phenomenon, a simple spring mass lumped parameter system is constructed as shown in Figure 142. Due to the speaker openings, extra connections are made between the main cavity and the trunk cavity which, in the finite element model is through off-diagonal spring elements. 
Therefore, though the cavity gets acoustically longer because of the speaker openings (due to which, the frequencies are expected to drop lower), due to the spring connections, the frequencies of the pure acoustic modes become higher.

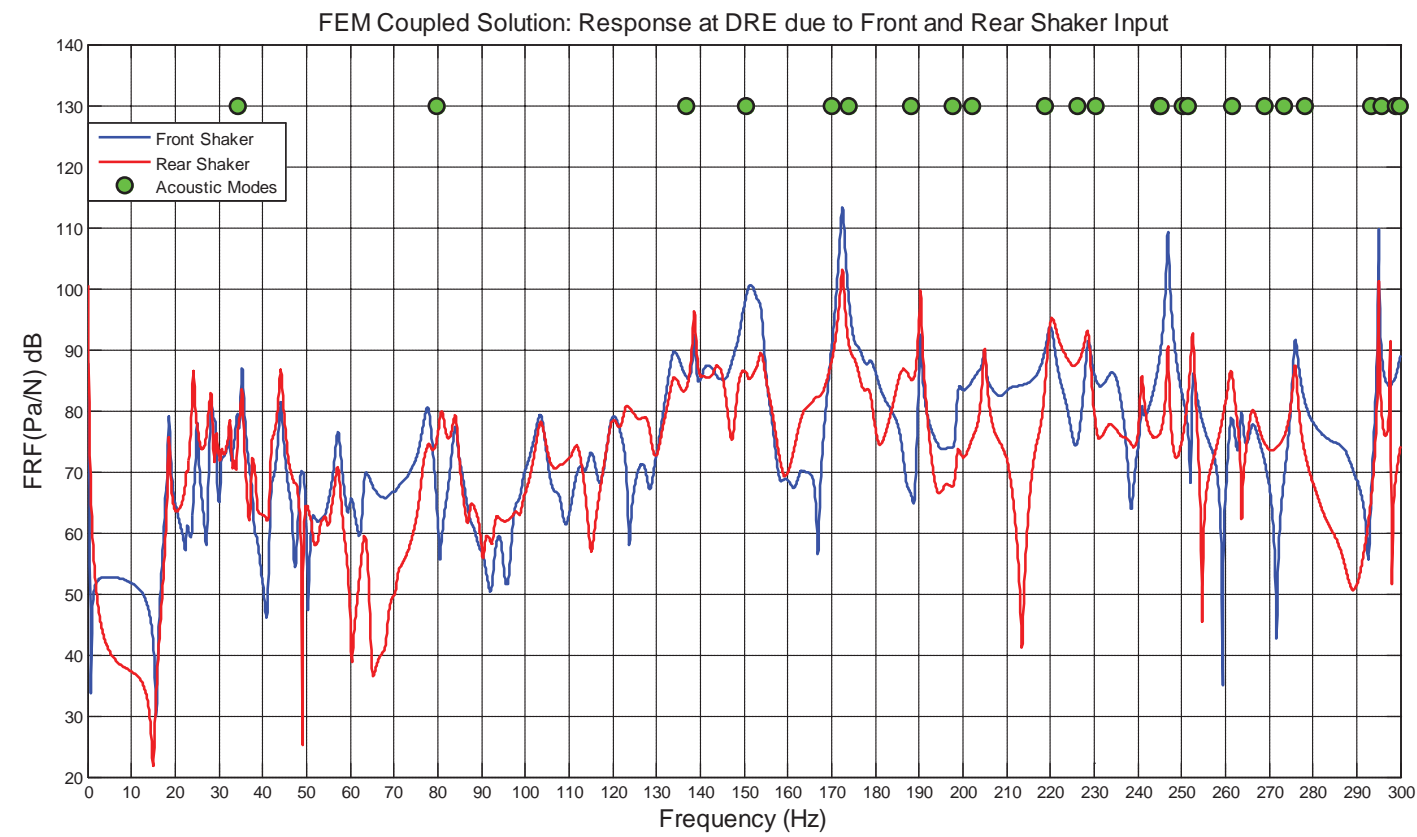

Figure 141: Acoustic Sensitivity at DRE due to front and rear shaker input for SCM 4B

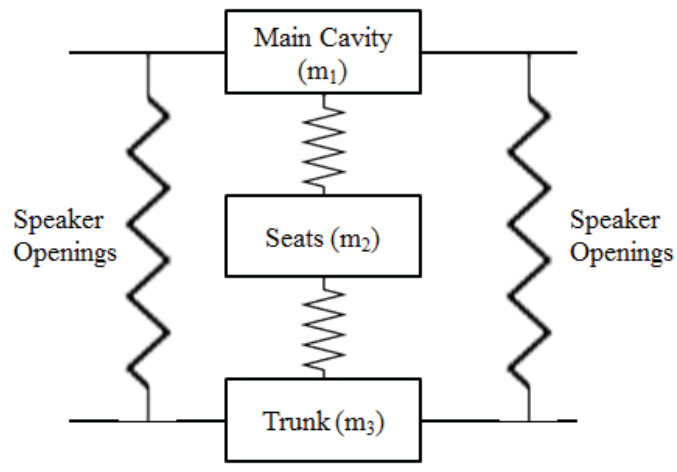

Figure 142: Spring Mass lumped parameter representation of SCM $4 B$

The comparison for acoustic sensitivity at the DRE between SCM4A and SCM4B is shown in Figure 143. The only difference between the two models is the speaker openings in the rear package shelf. The general trend in the acoustic sensitivity is similar; however, there is a significant shift in the acoustic modes. In the frequency range 90-130 $\mathrm{Hz}$ where no acoustic modes are present for both the models, the curves overlap each other. This shows the significance of acoustic modes and the overall fluid-structure 
interaction in the models. It can be seen that in the $50 \mathrm{~Hz}$ region, the response for SCM4B is higher because of the presence of the acoustic mode at $47.4 \mathrm{~Hz}$.
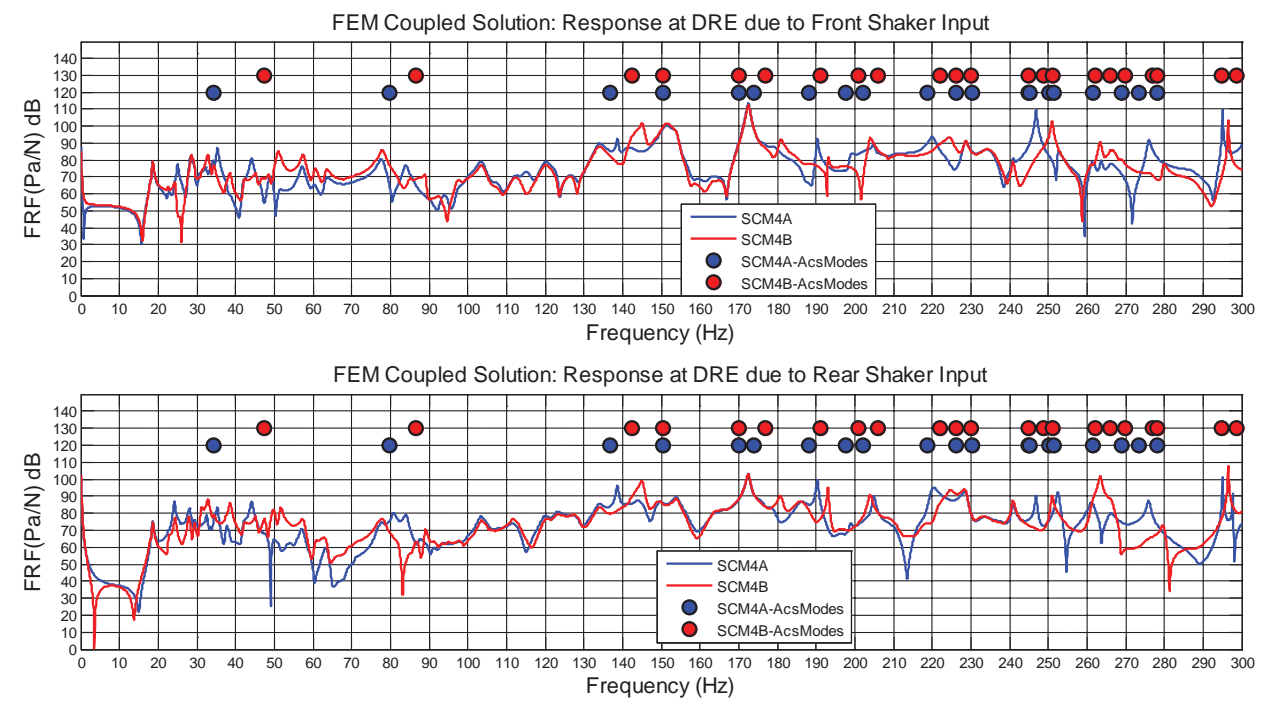

Figure 143: Acoustic Sensitivity Comparison between SCM4A and SCM4B

Figure 144 shows the MAC for SCM4B between the pure acoustic modes and coupled acoustic modes. Similar to SCM4A, high values of MAC are not seen. The trend is similar to the other simple car models. Based on this as well as the precious MACs, it is evident that the first few acoustic modes participate over a wide frequency range, especially for the boom frequencies. Changes in the structural modes, needs to be huge to offset the effect of the acoustic modes. However, from the acoustic sensitivity plots, it is seen that changes in the acoustic cavity (speaker openings, door cavity openings), significantly changes the response at DRE. Hence, to reduce the boom phenomenon, the approach of making changes to the acoustic cavity may also yield desirable results.

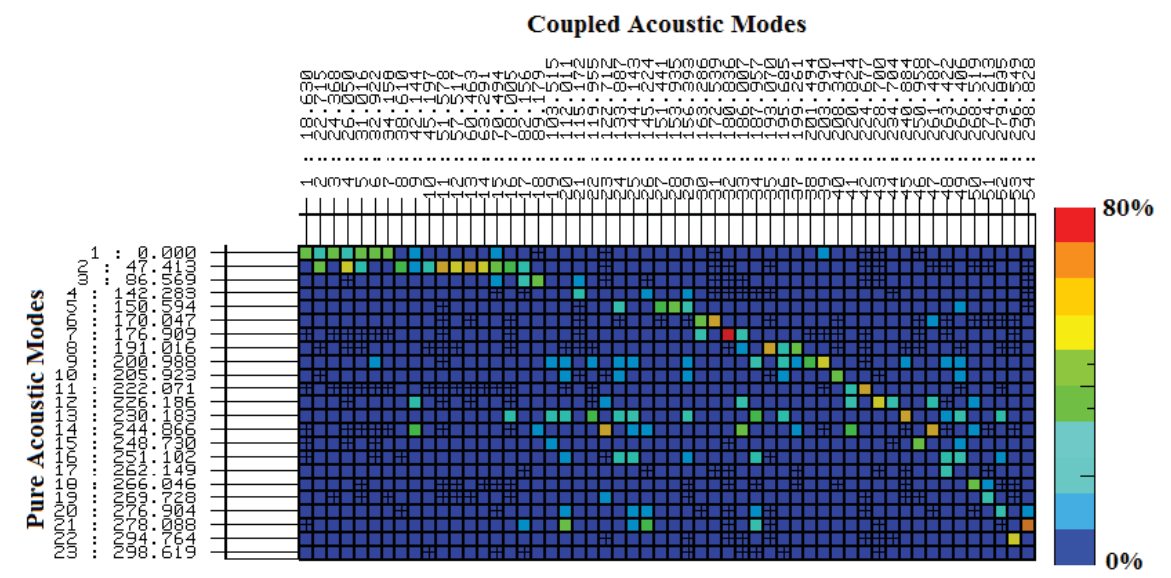

Figure 144: Acoustic MAC between Coupled Modes and Pure Acoustic Modes for SCM 4B 


\section{Conclusions and Recommendations}

With the help of multiple hardware tests and computer simulations, valuable information about the vibro-acoustic interaction between the vehicle structure and its cavity was obtained.

The hammer test successfully confirmed the presence of a boom mode at $40 \mathrm{~Hz}$. This result led to the structural full modal test. This test gave valuable information of the different structural modes participating in the boom range. The front header, rear header as well the trunk was identified as the major components that get excited in the boom frequency range. Pumping action due to these components resulted in the movement of air in the vehicle cabin resulting in boom.

Acoustic measurements due to the structural excitation revealed the acoustic response of the cabin and the trunk. For most of the modes, it looked like the structure was driving the fluid inside the vehicle.

Acoustic excitation of the vehicle with the help of speakers revealed that for most of the modes in the boom range, participation was from the first acoustic mode. This was later confirmed by the MAC plots of the simple car models as well.

Door cavity testing of the Cruze was performed to understand if they behaved like Helmholtz resonators as predicted by the finite element models. The response due to the front shaker suggested involvement of the door cavities in the acoustic sensitivity at the DRE. From the testing it was also observed that though the door cavities do not have a significant effect in the boom range, they do affect the sensitivities at other frequencies. The analytical models as well as the testing confirmed the need to model the door cavities.

Testing without the rear seats showed a reduced response due to lengthening of the acoustic cavity. The rear seats also behave as acoustic absorbers above the boom frequency range. The results in the boom frequency range suggested that the seats decouple the vehicle cabin and the trunk. This result was also achieved during the finite element analysis of the vehicle. The seatback testing showed a complex phenomenon between the acoustic pressure fluctuations and the seatback movement. The coupling between the structure and the fluid depended upon the mode being observed. Different modes showed different drivers.

Modeling and analysis of the simple car models helped in understanding the fluidstructure interaction. The FEM and BEM produced similar results for the uncoupled and coupled method. However, the BEM consumed a lot of time than FEM as the models were simple in construction. As the physical testing showed a two way interaction 
(coupled) between the structure and cabin, a FEM coupled approach was followed for the rest of the models. All the models showed the same trend for the acoustic MAC between the pure acoustic modes and the coupled acoustic modes. The first couple pure acoustic modes participated with the coupled modes for a wide frequency range. Hence, small changes in the structural members to shift the frequencies would not be enough to change the acoustic sensitivity. Significant changes in the structural models would be necessary. The seats behaved like acoustic absorbers and reduced the frequency of the acoustic modes by significant amount. The speaker openings behaved like additional spring connections between the main cavity and the trunk cavity by increasing the natural frequencies of the acoustic modes. For all the models, a high acoustic sensitivity was observed at the DRE at frequencies close to the natural frequencies of the acoustic modes. This confirmed the coupled phenomenon between the structure and acoustic cavity.

Based on the extensive testing of the actual vehicle and simulations run on the simple car models, following are the recommendations for modeling (for boom prediction) and possible reduction of the acoustic boom:

1. The front and rear headers as well as the trunk are important structural contributors in the boom range. Tuned mass dampers and/or Helmholtz resonators [15] should be built for these components to reduce their contribution in the boom range.

2. The testing as well as the simulation confirmed the presence of a two-way vibroacoustic coupling for the vehicle. Therefore, for modeling and prediction of the boom frequencies, a two-way coupled approach is highly recommended.

3. The FEM as well as BEM coupled approach resulted in the same acoustic sensitivity at the DRE till $300 \mathrm{~Hz}$ for Simple Car Model 1. Application of BEM approach and its comparison with the FEM results for the actual model is recommended.

4. The door cavities play an important role in the acoustic behavior of the vehicle. With one speaker opening, they behave like Helmholtz resonators and have significant effect on the acoustic sensitivity at DRE. Modeling of the door cavities is recommended while building the acoustic model.

5. The rear seats lower the acoustic modes of the vehicle significantly. From the acoustic mode shapes during testing, it is seen that the rear seats act as a barrier between the main cavity and the trunk cavity with the package shelf speakers as the only opening. For modeling the acoustic mesh, it is advised to connect the main cavity and trunk cavity by the speaker openings only while the seatback should be modeled as an acoustic barrier. 


\section{References}

1. Shorter, P., Recent advances in automotive interior noise prediction. 2008, SAE Technical Paper.

2. Dhandole, S. and S. Modak. Review of vibro-acoustics analysis procedures for prediction of low frequency noise inside a cavity. in Proceedings of the Conference \& Exposition on Structural Dynamics, Orlando, FL, USA. 2007.

3. Sol, A. and F. Van Herpe, Numerical prediction of a whole car vibro-acoustic behavior at low frequencies. 2001, SAE Technical Paper.

4. Tsuji, H., et al., A study of experimental acoustic modal analysis of automotive interior acoustic field coupled with the body structure. 2012, SAE Technical Paper.

5. W. Desmet, P.S., Introduction to Numerical Acoustics, in Numerical Acoustics Theoretical Manual. LMS International. p. 5-36.

6. Shreerang, D., A Study of Passenger Car Acoustic Cavity Boom Simulation Methods, in Department of Mechanical Engineering-Engineering Mechanics. 2014, Michigan Technological University: Houghton, MI.

7. Cherng, J.G., et al. Characterization and validation of acoustic cavities of automotive vehicles. in Society of Photo-optical Instrumentation Engineers. 2002.

8. Desmet, W., Boundary Element Method for Acoustics, in Numerical Acoustics Theoretical Manual. p. 86-126.

9. W. Desmet, D.V., Finite Element Modeling for Acoustics, in Numerical Acoustics Theoretical Manual. LMS International. p. 37-85.

10. Gérard, F., et al., Acoustic transfer vectors for numerical modeling of engine noise. Sound and Vibration, 2002. 36(7): p. 20-25.

11. McCulloch, C., M. Tournour, and P. Guisset, Modal Acoustic Transfer Vectors Make Acoustic Radiation Models Practical for Engines and Rotating Machinery. LMS International, Leuven, Belgium.

12. Citarella, R., L. Federico, and A. Cicatiello, Modal acoustic transfer vector approach in a FEM-BEM vibro-acoustic analysis. Engineering Analysis with Boundary Elements, 2007. 31(3): p. 248-258.

13. Karsen, C.V., Experimental Vibro-Acoustics. 2012.

14. LMS Test.Lab Documentation.

15. Kashani, R. and J. Orzechowski, Active boom noise damping of dodge durango. 2001, SAE Technical Paper. 